\title{
Analysis of composite moving beams using higher order shear deformation theory
}

\author{
Govindan Nagappan \\ West Virginia University
}

Follow this and additional works at: https://researchrepository.wvu.edu/etd

\section{Recommended Citation}

Nagappan, Govindan, "Analysis of composite moving beams using higher order shear deformation theory" (2004). Graduate Theses, Dissertations, and Problem Reports. 1451.

https://researchrepository.wvu.edu/etd/1451

This Thesis is protected by copyright and/or related rights. It has been brought to you by the The Research Repository @ WVU with permission from the rights-holder(s). You are free to use this Thesis in any way that is permitted by the copyright and related rights legislation that applies to your use. For other uses you must obtain permission from the rights-holder(s) directly, unless additional rights are indicated by a Creative Commons license in the record and/ or on the work itself. This Thesis has been accepted for inclusion in WVU Graduate Theses, Dissertations, and Problem Reports collection by an authorized administrator of The Research Repository @ WVU. For more information, please contact researchrepository@mail.wvu.edu. 


\title{
Analysis of Composite Moving Beams using Higher Order Shear Deformation Theory
}

\section{Govindan Nagappan}

Thesis submitted to the

\section{College of Engineering and Mineral Resources}

at West Virginia University in partial fulfillment of the requirements

for the degree of

\author{
Master of Science \\ in \\ Mechanical Engineering \\ Nithi T. Sivaneri, Ph.D., Chair \\ Jacky C. Prucz, Ph.D., \\ Bruce S. Kang, Ph.D.,
}

Department of Mechanical and Aerospace Engineering

\author{
Morgantown, West Virginia \\ 2004
}

Keywords: Composite Materials, Moving Beam, HSDT, Finite Element Method 


\section{ABSTRACT \\ Analysis of Composite Moving Beams using Higher Order Shear Deformation Theory}

Govindan Nagappan

A Composite beam moving longitudinally over its supports is analyzed based on a higher-order shear deformation theory. The dynamic behavior of the beam is studied using a finite element formulation based on a variational principle. The essential constraints are applied via Lagrange multipliers and this method is effective for the moving beam problem in which the support locations relative to the beam change with time and do not always fall exactly at the nodes. An initially deformed overhang beam moving over two simple supports is used for the analysis. The first flexural mode shape of the beam is used as the initial shape of the beam. The finite element equations of motion are then solved using time integration methods such as Newmark's method and Wilson Theta method. The results are presented in terms of time history of tip deflections. The performance of the higher-order shear deformation model is compared with that of the first-order shear deformation theory and Classical laminate plate theory. The response in all the cases exhibits a beat-like phenomenon due to the interplay between the axial forcing frequency and the transverse natural frequencies. In all the cases considered, the axial motion causes a magnification in the transverse deflection by about $40 \%$. 


\section{ACKNOWLEDGEMENTS}

I would like to express my sincere gratitude and appreciation to my research advisor Dr. Nithi Sivaneri for his invaluable guidance and support during the entire course of my project. I would like to thank my committee members Dr. Jacky Prucz and Dr. Bruce Kang for their valuable suggestions. I wish to express my sincere and heartfelt thanks to my parents, for their love and support throughout the course of my education. I wish to express my appreciation to all my friends and roommates for their support and encouragement. Last but not the least I would like to thank the Almighty for showing me the right path in life. 


\section{TABLE OF CONTENTS}

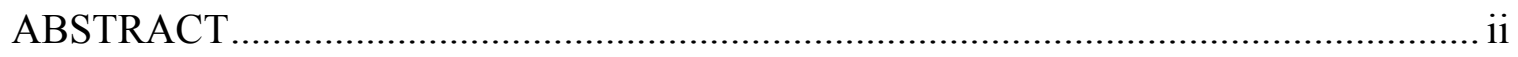

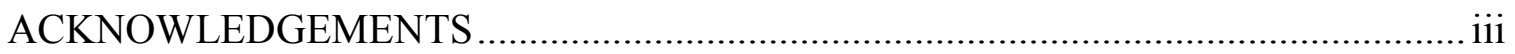

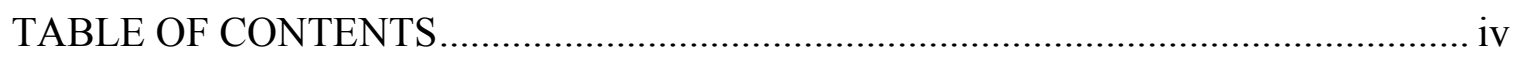

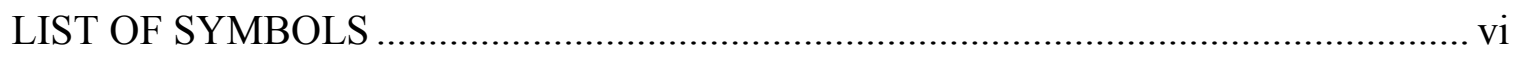

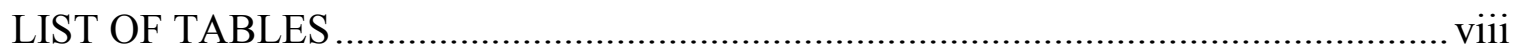

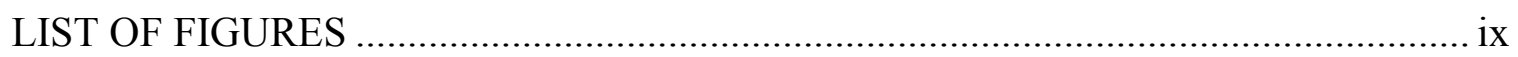

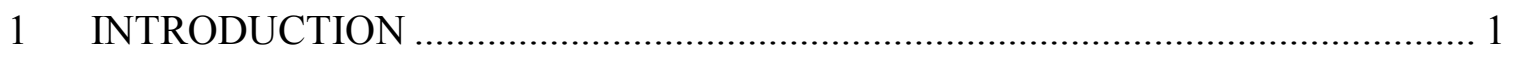

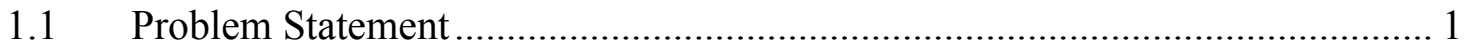

1.2 Laminated Composites....................................................................................... 1

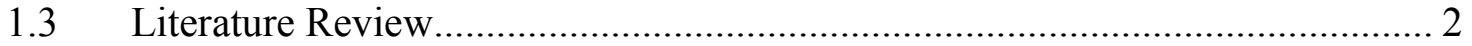

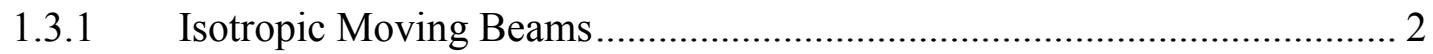

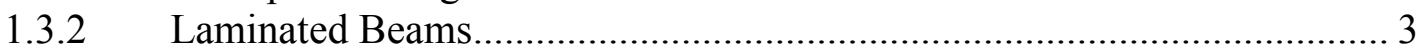

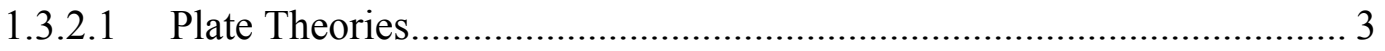

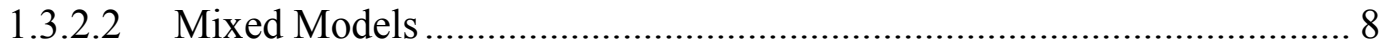

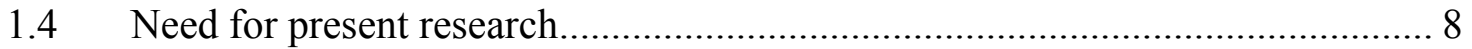

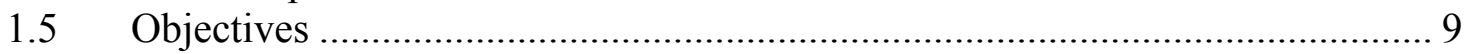

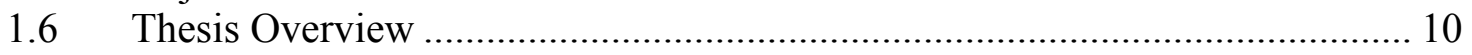

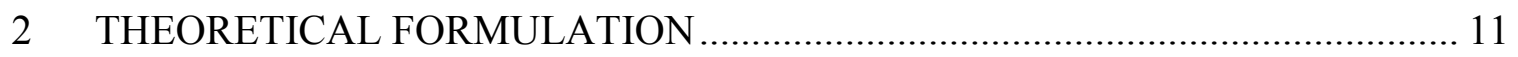

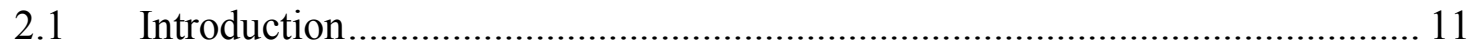

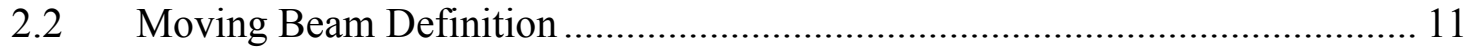

2.2.1 Lay-up Configuration......................................................................... 12

2.2.2 Force and Moment Resultants ............................................................. 13

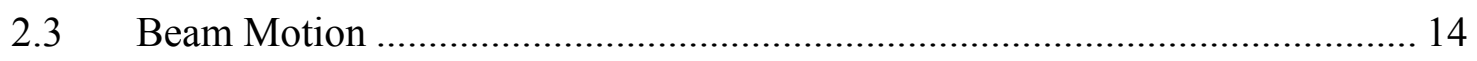

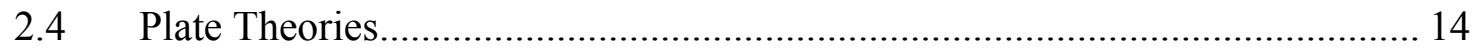

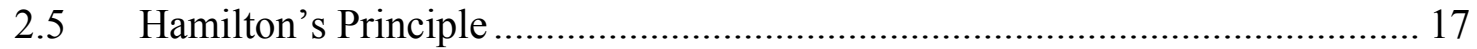

2.5.1 Kinematics Equations of a composite plate ............................................. 18

2.5.2 Virtual Strain Energy for a Plate........................................................... 21

2.5.3 Constitutive Equations ........................................................................... 22

2.5.4 Reduction of Plate Equations to Beam ……………………………….... 23

2.5.5 Virtual Strain Energy for a Beam ...................................................... 25

2.5.6 Virtual Kinetic Energy for a Beam ......................................................... 26

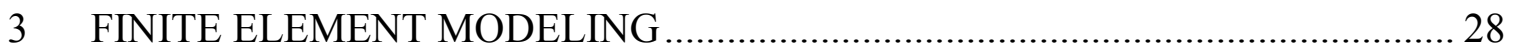

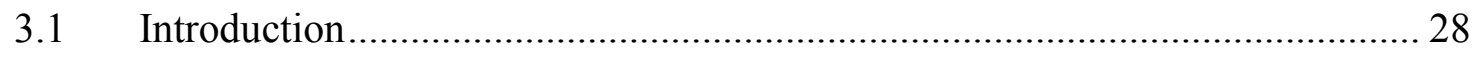

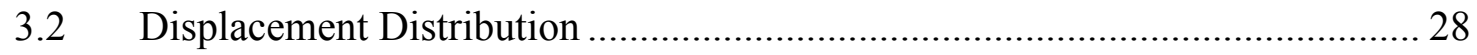

3.3 Element Stiffness Matrix Fornulation......................................................... 33

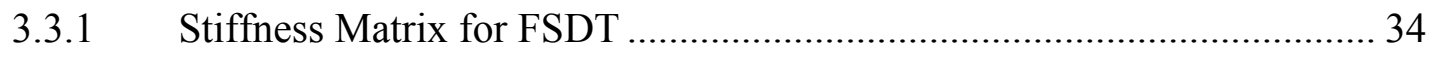

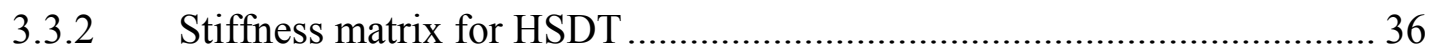




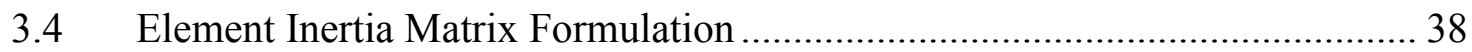

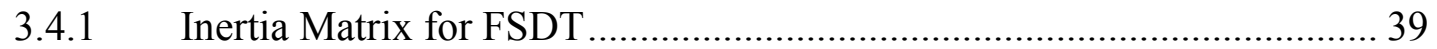

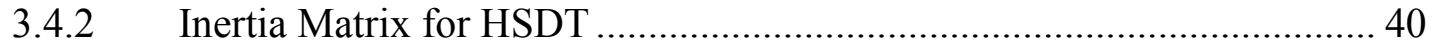

3.5 Global Equations of Motion......................................................................... 42

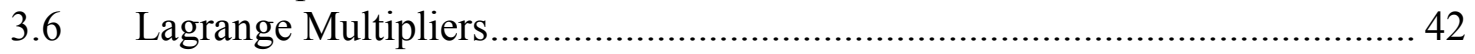

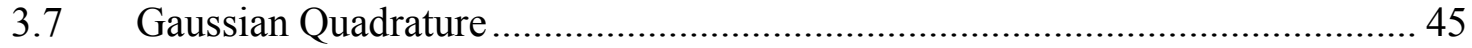

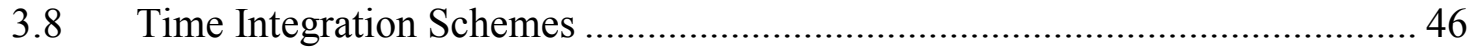

3.9 Boundary Conditions and Initial Conditions .................................................... 48

3.9.1 Boundary Conditions for CLPT ........................................................... 48

3.9.2 Boundary Conditions for FSDT ............................................................ 48

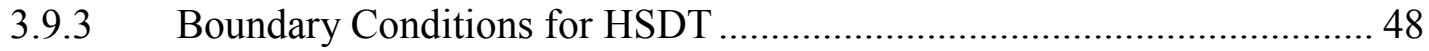

3.9.4 Initial Conditions …………………........................................................ 49

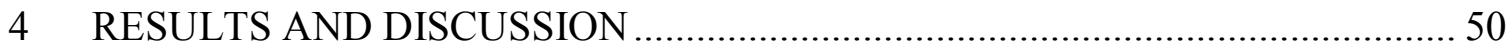

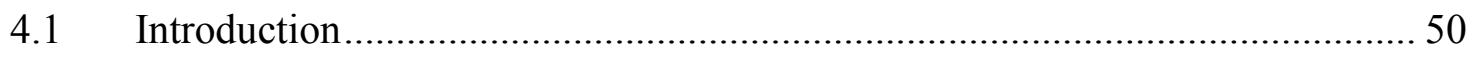

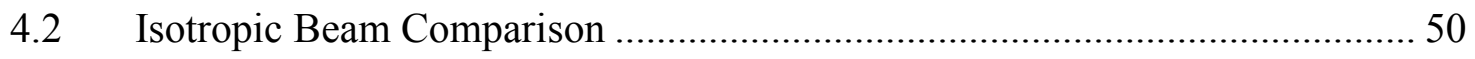

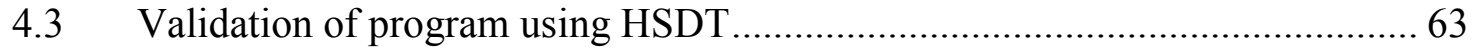

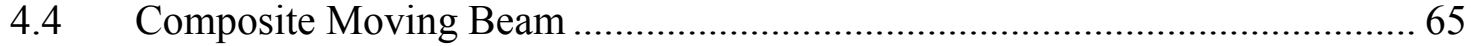

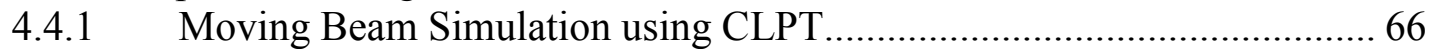

4.4.2 Moving Beam Simulation using FSDT ................................................... 80

4.4.3 Moving Beam Simulation using HSDT .................................................. 82

5 CONCLUSIONS AND RECOMMENDATIONS …………………………........ 120

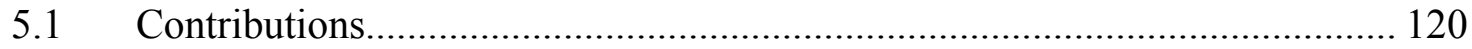

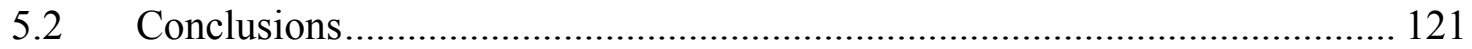

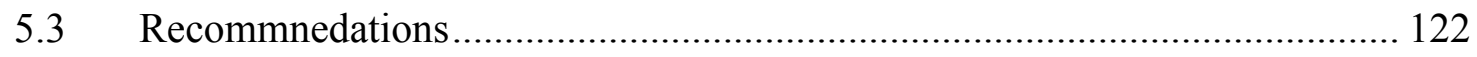

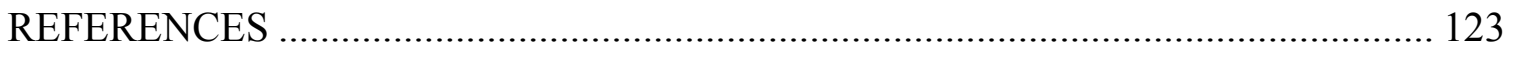

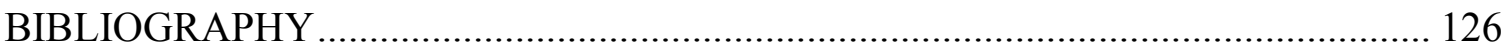




\section{LIST OF SYMBOLS}

\begin{tabular}{|c|c|}
\hline$a_{B}^{L}$ & - Longitudinal rigid-body acceleration of the beam $\left(\mathrm{m} / \mathrm{s}^{2}\right)$ \\
\hline$A$ & - Amplitude of longitudinal rigid-body motion (m) \\
\hline$[A]$ & - Axial stiffness matrix $(\mathrm{N} / \mathrm{m})$ \\
\hline$a_{i}, b_{j}$ & - Generalized coordinates \\
\hline$b$ & - Width of the beam (m) \\
\hline$[B]$ & - Bending-extension stiffness matrix $(\mathrm{N})$ \\
\hline$d$ & - Distance between supports (m) \\
\hline$[D]$ & - Bending stiffness matrix (Nm) \\
\hline$E$ & - Modulus of elasticity $\left(\mathrm{N} / \mathrm{m}^{2}\right)$ \\
\hline$[E],[F],[H]$ & - Higher-order stiffness matrices $\left(\mathrm{Nm}^{2}, \mathrm{Nm}^{3}, \mathrm{Nm}^{5}\right)$ \\
\hline$h$ & - Thickness of the beam (m) \\
\hline$H_{i}, H_{L i}$ & - Hermitian and Lagrangian Shape functions \\
\hline $\begin{array}{l}I \\
I_{0}, I_{1}, I_{2}\end{array}$ & $\begin{array}{l}\text { - Moment of Inertia }\left(\mathrm{m}^{4}\right) \\
\text { - Normal, coupled normal-rotary, and rotary inertia coefficients }\end{array}$ \\
\hline$I_{3}, I_{4}, I_{6}$ & - Higher-order inertia coefficients \\
\hline$\left[K_{x x}\right]$ & - Finite element stiffness matrix for composite material $(\mathrm{N} / \mathrm{m})$ \\
\hline$[K]$ & - Global stiffness matrix $(\mathrm{N} / \mathrm{m})$ \\
\hline$\left[K_{\lambda}\right]$ & - Lagrange multiplier matrix $(\mathrm{N} / \mathrm{m})$ \\
\hline$L$ & - Length of the beam (m) \\
\hline$l_{e}$ & - Element length (m) \\
\hline$\left[M_{x x}\right]$ & - Finite element inertia matrix for composite material \\
\hline$[M]$ & - Global Inertia matrix \\
\hline$M_{x}, M_{y}, M_{x y}$ & - In plane moment resultants in $x y$ plane $(\mathrm{Nm})$ \\
\hline$n$ & - Number of layers \\
\hline$N_{x}, N_{y}, N_{x y}$ & - In plane force resultants in $x y$ plane $(\mathrm{N} / \mathrm{m})$ \\
\hline$P_{x}, P_{y}, P_{x y}$ & - Higher order stress resultants \\
\hline & - Global displacements vector (m) \\
\hline$Q_{x}, Q_{y}$ & - Transverse shear force resultants $(\mathrm{N} / \mathrm{m})$ \\
\hline$\left[\bar{Q}^{k}\right]$ & - Transformed reduced stiffness matrix of $k^{\text {th }}$ layer $\left(\mathrm{N} / \mathrm{m}^{2}\right)$ \\
\hline$\{Q\}$ & - Global load vector $(\mathrm{N})$ \\
\hline$[\mathrm{S}]^{\mathrm{ij}}$ & - Partitions of reduced $[\mathrm{ABD}]$ matrix in formulation using FSDT \\
\hline$S_{i j}$ & - Element of reduced $[\mathrm{S}]$ matrix in formulation based on FSDT \\
\hline$t$ & - Time (sec) \\
\hline$t_{k}$ & - Thickness of $k^{\text {th }}$ layer (m) \\
\hline$T$ & - Total kinetic energy $(\mathrm{Nm})$ \\
\hline
\end{tabular}




\begin{tabular}{|c|c|}
\hline$[T]^{i j}$ & - Partitions of reduced [ABDEF] matrix in formulation using \\
\hline & HSDT \\
\hline$T_{i j}$ & - Elements of reduced [T] matrix in formulation based on HSDT \\
\hline$u$ & - Axial deflection (m) \\
\hline$u_{0}, v_{0}, w_{0}$ & - Mid-plane displacements along $x, y$, and $z$ axis, respectively (m) \\
\hline$U$ & - Total strain energy $(\mathrm{Nm})$ \\
\hline$V$ & - Volume $\left(\mathrm{m}^{3}\right)$ \\
\hline$V_{B}^{L}$ & - Longitudinal rigid-body velocity of the beam $(\mathrm{m} / \mathrm{s})$ \\
\hline$w_{b}, w_{s}$ & - Shear and bending components of transverse deflection (m) \\
\hline$w$ & - Transverse deflection $(\mathrm{m})$ \\
\hline$W$ & - Work done (Nm) \\
\hline$x_{e}$ & - Element longitudinal axis \\
\hline$X_{A}(t)$ & - Axial displacement of the moving beam \\
\hline$X, Y$ & - Global Axes \\
\hline$z$ & - Thickness coordinate \\
\hline$z_{k}$ & $\begin{array}{l}\text { - Distance to the top of the } k^{\text {th }} \text { layer from the mid-plane of a } \\
\text { laminate }(\mathrm{m})\end{array}$ \\
\hline$\overline{z_{k}}$ & $\begin{array}{l}\text { - Distance to the mid surface of the } k^{\text {th }} \text { layer from the mid-plane of } \\
\text { a laminate }(\mathrm{m})\end{array}$ \\
\hline $\bar{\Delta}_{p}$ & - Hamiltonian function \\
\hline$\Omega$ & $\begin{array}{l}\text { - Frequency of rigid-body axial motion imparted to the beam } \\
(\mathrm{rad} / \mathrm{sec})\end{array}$ \\
\hline$\delta()$ & - Variation of ( ) \\
\hline$\varepsilon_{x}, \varepsilon_{y}, \varepsilon_{z}$ & - Normal strains in $x, y$, and $z$, directions, respectively \\
\hline$\phi_{x}, \phi_{y}$ & - Rotations about $x$ and $y$, respectively \\
\hline$\gamma_{x y}, \gamma_{y z}, \gamma_{x z}$ & - Engineering shear strains \\
\hline$\rho$ & - Mass Density $\left(\mathrm{Kg} / \mathrm{m}^{3}\right)$ \\
\hline$\xi$ & - Element non-dimensional coordinate \\
\hline()$^{T}$ & - Transpose of ( ) \\
\hline()$\left.^{\prime}\right),(")$ & - First and second partial derivatives with respect to time \\
\hline()$^{\prime},()^{\prime \prime}$ & - First and second partial derivatives with respect to $x$ \\
\hline()$^{y}$ & - Partial derivative with respect to $y$ \\
\hline
\end{tabular}




\section{LIST OF TABLES}

Table 3.1 Gauss integration points and weights .................................................. 46

Table 4.1 Summary of steel moving beam response ................................................. 54

Table 4.2 Maximum tip deflection of steel beam ..................................................... 54

Table 4.3 Transverse deflections of composite beam obtained using HSDT ................. 64

Table 4.4 Non-dimensional fundamental frequencies of composite beam.................... 65

Table 4.5 Maximum tip deflections of Beams based on CLPT ...................................... 68

Table 4.6 Instantaneous first natural frequency of beams based on CLPT ..................... 69

Table 4.7 Maximum tip deflections of beams based on FSDT ................................... 81

Table 4.8 Instantaneous first natural frequency of beams based on FSDT .................... 82

Table 4.9 Maximum tip deflections of beam based on HSDT …................................ 101

Table 4.10 Instantaneous first natural frequency of beams based on HSDT................ 102 


\section{LIST OF FIGURES}

Figure 2.1 Coordinate systems for the moving beam 11

Figure 2.2 Composite lay-up configuration $\quad 13$

Figure 2.3 Force and Moment Resultants on a flat plate [Barbero (1998)] 13

Figure 2.4 Deformation of transverse normal for CLPT, FSDT and HSDT [Reddy (1997)] 16

Figure 3.1 Element definition for formulation using HSDT 29

Figure 4.1 Isotropic Moving Beam $\quad 50$

Figure 4.2 Response analysis of initially deformed isotropic moving beam with $\Omega=10$ $\mathrm{rad} / \mathrm{sec} \quad 55$

Figure 4.3 Response analysis of initially deformed isotropic moving beam with $\Omega=20$ $\mathrm{rad} / \mathrm{sec} \quad 55$

Figure 4.4 Response analysis of initially deformed isotropic moving beam with $\Omega=22$ $\mathrm{rad} / \mathrm{sec} \quad 56$

Figure 4.5 Response analysis of initially deformed isotropic moving beam with $\Omega=30$ $\mathrm{rad} / \mathrm{sec} \quad 56$

Figure 4.6 Response of initially deformed steel beam oscillating axially with $\Omega=0$ $\mathrm{rad} / \mathrm{sec} \quad 57$

Figure 4.7 First transverse natural frequency of initially deformed steel beam oscillating axially with $\Omega=0 \mathrm{rad} / \mathrm{sec} \quad 57$

Figure 4.8 Response of initially deformed steel beam oscillating axially with $\Omega=5$ $\mathrm{rad} / \mathrm{sec} \quad 58$

Figure 4.9 First transverse natural frequency of initially deformed steel beam oscillating axially with $\Omega=5 \mathrm{rad} / \mathrm{sec} \quad 58$

Figure 4.10 Response of initially deformed steel beam oscillating axially with $\Omega=10$ $\mathrm{rad} / \mathrm{sec}$

Figure 4.11 First transverse natural frequency of initially deformed steel beam oscillating axially with $\Omega=10 \mathrm{rad} / \mathrm{sec}$

Figure 4.12 Response of initially deformed steel beam oscillating axially with $\Omega=15$ $\mathrm{rad} / \mathrm{sec}$

Figure 4.13 First transverse natural frequency of initially deformed steel beam oscillating axially with $\Omega=15 \mathrm{rad} / \mathrm{sec}$

Figure 4.14 Response of initially deformed steel beam oscillating axially with $\Omega=20$ $\mathrm{rad} / \mathrm{sec}$

Figure 4.15 First transverse natural frequency of initially deformed steel beam oscillating axially with $\Omega=20 \mathrm{rad} / \mathrm{sec}$

Figure 4.16 Response of initially deformed steel beam oscillating axially with $\Omega=25$ $\mathrm{rad} / \mathrm{sec}$

Figure 4.17 First transverse natural frequency of initially deformed steel beam oscillating axially with $\Omega=25 \mathrm{rad} / \mathrm{sec}$

Figure 4.18 Transverse left tip deflection $w$, CLPT, $\Omega=10 \mathrm{rad} / \mathrm{sec} \quad 70$

Figure 4.19 Axial right tip deflection $u$, CLPT, $\Omega=10 \mathrm{rad} / \mathrm{sec} \quad 71$

Figure 4.20 Transverse left tip deflection $w$, CLPT, $\Omega=20 \mathrm{rad} / \mathrm{sec} \quad 72$

Figure 4.21 Transverse left tip deflection $w$, CLPT, $\Omega=10 \mathrm{rad} / \mathrm{sec} \quad 73$ 
Figure 4.22 Transverse left tip deflection $w$, CLPT, $\Omega=20 \mathrm{rad} / \mathrm{sec} \quad 74$

Figure 4.23 Transverse left tip deflection $w$, CLPT, $\Omega=20 \mathrm{rad} / \mathrm{sec} \quad 75$

Figure 4.24 Axial right tip deflection $u$, CLPT, $\Omega=20 \mathrm{rad} / \mathrm{sec}$

Figure 4.25 First transverse natural frequency $\omega_{1}$, of [0/+45], CLPT, $\Omega=10 \mathrm{rad} / \mathrm{sec} 77$

Figure 4.26 First transverse natural frequency $\omega_{1}$, of $[0 /+45]$, CLPT, $\Omega=20 \mathrm{rad} / \mathrm{sec} \quad 77$

Figure 4.27 First transverse natural frequency $\omega_{1}$, of $[0 /+20]$, CLPT, $\Omega=10 \mathrm{rad} / \mathrm{sec} \quad 78$

Figure 4.28 First transverse natural frequency $\omega_{1}$, of $[0 /+20]$, CLPT, $\Omega=20 \mathrm{rad} / \mathrm{sec} \quad 78$

Figure 4.29 First transverse natural frequency $\omega_{1}$, of $[0 /+45]_{\mathrm{s}} \mathrm{CLPT}, \Omega=10 \mathrm{rad} / \mathrm{sec} \quad 79$

Figure 4.30 Transverse left tip deflection $w_{b}$, FSDT, $\Omega=10 \mathrm{rad} / \mathrm{sec} \quad 83$

Figure 4.31 Transverse left tip deflection $w_{s}$, FSDT, $\Omega=10 \mathrm{rad} / \mathrm{sec} \quad 84$

Figure 4.32 Transverse left tip deflection $w_{b}$, FSDT, $\Omega=20 \mathrm{rad} / \mathrm{sec} \quad 85$

Figure 4.33 Transverse left tip deflection $w_{s}$, FSDT, $\Omega=20 \mathrm{rad} / \mathrm{sec} \quad 86$

Figure 4.34 Transverse left tip deflection $w$, FSDT, $\Omega=20 \mathrm{rad} / \mathrm{sec} \quad 87$

Figure 4.35 Axial right tip deflection $u$, FSDT, $\Omega=20 \mathrm{rad} / \mathrm{sec} \quad 88$

Figure 4.36 Left tip shear strain $\gamma$, FSDT, $\Omega=20 \mathrm{rad} / \mathrm{sec} \quad 88$

Figure 4.37 Left tip slope $w_{b}$ ', FSDT, $\Omega=20 \mathrm{rad} / \mathrm{sec} \quad 89$

Figure 4.38 Left tip slope $w_{b}{ }^{y}$, FSDT, $\Omega=20 \mathrm{rad} / \mathrm{sec} \quad 90$

Figure 4.39 Transverse left tip deflection $w_{b}$, FSDT, $\Omega=10 \mathrm{rad} / \mathrm{sec}$

Figure 4.40 Transverse left tip deflection $w_{s}$, FSDT, $\Omega=10 \mathrm{rad} / \mathrm{sec} \quad 92$

Figure 4.41 Transverse left tip deflection $w_{b}$, FSDT, $\Omega=20 \mathrm{rad} / \mathrm{sec} \quad 93$

Figure 4.42 Transverse Left tip deflection, $w_{s}$, FSDT, $\Omega=20 \mathrm{rad} / \mathrm{sec} \quad 94$

Figure 4.43 Transverse left tip deflection $w_{b}$, FSDT, $\Omega=20 \mathrm{rad} / \mathrm{sec}$

Figure 4.44 Transverse left tip deflection, $w_{s}$, FSDT, $\Omega=20 \mathrm{rad} / \mathrm{sec} \quad 96$

Figure 4.45 First transverse natural frequency $\omega_{1}$, of $[0 /+45]$, FSDT, $\Omega=10 \mathrm{rad} / \mathrm{sec} \quad 97$

Figure 4.46 First transverse natural frequency $\omega_{1}$, of $[0 /+45]$, FSDT, $\Omega=20 \mathrm{rad} / \mathrm{sec} \quad 97$

Figure 4.47 First transverse natural frequency $\omega_{1}$, of [0/+20], FSDT, $\Omega=10 \mathrm{rad} / \mathrm{sec} \quad 98$

Figure 4.48 First transverse natural frequency $\omega_{1}$, of [0/+20], FSDT, $\Omega=20 \mathrm{rad} / \mathrm{sec} \quad 98$

Figure 4.49 First transverse natural frequency $\omega_{1}$, of $[0 /+45]_{\mathrm{s}}$ FSDT, $\Omega=10 \mathrm{rad} / \mathrm{sec} \quad 99$

Figure 4.50 Transverse left tip deflection $w_{b}, \mathrm{HSDT}, \Omega=10 \mathrm{rad} / \mathrm{sec} \quad 103$

Figure 4.51 Transverse left tip deflection $w_{s}$, HSDT, $\Omega=10 \mathrm{rad} / \mathrm{sec} \quad 104$

Figure 4.52 Transverse left tip deflection $w_{b}$, HSDT, $\Omega=20 \mathrm{rad} / \mathrm{sec} \quad 105$

Figure 4.53 Transverse left tip deflection $w_{s}$, HSDT, $\Omega=20 \mathrm{rad} / \mathrm{sec} \quad 106$

Figure 4.54 Transverse left tip deflection $w$, HSDT, $\Omega=20 \mathrm{rad} / \mathrm{sec} \quad 107$

Figure 4.55 Axial right tip deflection $u$, HSDT, $\Omega=20 \mathrm{rad} / \mathrm{sec} \quad 108$

Figure 4.56 Left tip shear strain $\gamma$, HSDT, $\Omega=20 \mathrm{rad} / \mathrm{sec} \quad 108$

Figure 4.57 Left tip slope $w_{b}$ ', HSDT, $\Omega=20 \mathrm{rad} / \mathrm{sec} \quad 109$

Figure 4.58 Left tip slope $w_{s}{ }^{y}$, HSDT, $\Omega=20 \mathrm{rad} / \mathrm{sec} \quad 110$

Figure 4.59 Transverse left tip deflection $w_{b}, \mathrm{HSDT}, \Omega=10 \mathrm{rad} / \mathrm{sec} \quad 111$

Figure 4.60 Transverse left tip deflection $w_{s}$, HSDT, $\Omega=10 \mathrm{rad} / \mathrm{sec} \quad 112$

Figure 4.61 Transverse left tip deflection $w_{b}$, HSDT, $\Omega=20 \mathrm{rad} / \mathrm{sec} \quad 113$

Figure 4.62 Transverse Left tip deflection, $w_{s}$, HSDT, $\Omega=20 \mathrm{rad} / \mathrm{sec} \quad 114$

Figure 4.63 Transverse left tip deflection $w_{b}$, HSDT, $\Omega=20 \mathrm{rad} / \mathrm{sec} \quad 115$

Figure 4.64 Transverse left tip deflection, $w_{s}$, HSDT, $\Omega=20 \mathrm{rad} / \mathrm{sec} \quad 116$

Figure 4.65 First transverse natural frequency $\omega_{1}$, of $[0 /+45]$, HSDT, $\Omega=10 \mathrm{rad} / \mathrm{sec} 117$

Figure 4.66 First transverse natural frequency $\omega_{1}$, of $[0 /+45]$, HSDT, $\Omega=20 \mathrm{rad} / \mathrm{sec} 117$

Figure 4.67 First transverse natural frequency $\omega_{1}$, of $[0 /+20]$, HSDT, $\Omega=10 \mathrm{rad} / \mathrm{sec} 118$ 
Figure 4.68 First transverse natural frequency $\omega_{1}$, of [0/+20], HSDT, $\Omega=20 \mathrm{rad} / \mathrm{sec} 118$ Figure 4.69 First transverse natural frequency $\omega_{1}$, of $[0 /+45]_{\mathrm{s}} \mathrm{HSDT}, \Omega=10 \mathrm{rad} / \mathrm{sec} 119$ 


\section{INTRODUCTION}

\subsection{Problem Statement}

Beams moving over supports have applications in the fields of robotics, structural and earthquake engineering. The dynamic behavior of moving beams has been studied in the past. The vibration characteristics of beams made of isotropic and laminated composite materials based on Classical Laminate Plate Theory (CLPT) and First-order Shear Deformation Theory (FSDT) have been analyzed. The CLPT neglects transverse shear deformation which may be more important for composite beams than isotropic beams. The FSDT uses a shear correction factor, which is only an approximation. Therefore it is expected that, a Higher-order Shear Deformation Theory (HSDT) may overcome the disadvantages of CLPT and FSDT. In this research the dynamic behavior of a composite moving beam is analyzed by formulating and solving finite element equations based on HSDT.

\subsection{Laminated Composites}

Composite materials are a combination of two or more materials designed to provide better engineering properties compared to conventional materials. Composite materials can be classified into three types namely fibrous composites, particulate composites and laminated composites. Some advantages of composite materials are their high stiffness-to-weight ratio and strength-to-weight ratio and the ability to incorporate material design in the design process of the component or structure.

A fiber-reinforced lamina is a sheet of composite material with fibers embedded in a matrix material. A lamina has maximum strength in the fiber direction and it is weak 
in the other directions. Stacking several laminae in order to produce desired strength and stiffness properties forms a laminate. Laminated composites are generally classified into two types based on their lay-up properties. They are symmetric and unsymmetric lay-ups. A laminate is said to be symmetric if it has the same number of layers with the same orientation and thickness located symmetrically about the mid-plane; otherwise it is unsymmetric.

Laminated composites are usually treated as plate elements. This is because composites have their planar dimensions comparatively larger than the thickness. Therefore, to study the behavior of composites, laminate plate theories were developed [Reddy (1985)]. When the width of the plate is small compared to the length it is treated as a beam. Reddy (1985) presents analytical solutions for a number of laminated beams and plate strips. These solutions can be used as a basis for analyzing more complicated problems.

\subsection{Literature Review}

\subsubsection{Isotropic Moving Beams}

Buffinton and Kane (1985) studied the response of an isotropic beam moving over bilateral supports. The governing equations were set up considering the supports as kinematical constraints. The beam was discretized using the assumed-modes technique. The equation thus formulated was incorporated into a numerical procedure and the response of the beam for different types of longitudinal motion was studied.

Lee, H.P. (1992) also studied the response of beams moving over multiple supports using the assumed-modes technique to solve the governing equation. The 
governing equation was formulated based on Hamilton's principle. Numerical simulations were performed to study the response for different types of beam motion. Fourth-order Runge-Kutta method was used for numerical integration. The first symmetric flexural mode shape of the beam was used as the initial shape. This was different from the shape used by Buffinton and Kane; Buffinton and Kane (1985) found the deflection of the beam due to a statically applied uniform load and performed curve fitting to generate the initial shape. Lee avoided the curve-fitting step, which used an approximate function for generating the initial shape. Sreeram and Sivaneri (1997) applied the finite element method to study the response of an isotropic moving beam. The governing equation was formulated based on the variational principle. An h-p version finite element model was developed. The essential conditions were applied via Lagrange multipliers. The results were compared with that of Buffinton and Kane (1985) and Lee (1992). A convergence study was performed to determine the number of degrees of freedom required to produce a reasonably accurate solution. The time integration was carried out using numerical methods such as Wilson theta method, Newmark's method, Houbolt's method and the central difference method. Wilson theta method and Newmark's method produced more accurate results than the other methods.

\subsubsection{Laminated Beams}

\subsubsection{Plate Theories}

Reddy (1985) listed various theories that can be used for the analysis of composite plates. One of the earliest analyses was based on a three-dimensional elasticity theory. According to this theory, every layer is considered as an elastic continuum with 
distinct material properties from adjacent layers. Later lamination theories were developed considering the laminate to be in a state of plane stress resulting in the Classical Lamination Plate Theory (CLPT). The CLPT ignores transverse stress components and therefore was found to be inadequate for analysis of composite plates. To overcome this disadvantage, a first-order shear-deformation theory was developed. This displacement-based shear-deformation theory has become popular since it accounts for the transverse shear stresses. The differential equations for these theories were derived based on methods such as Castigliano's theorem, Principle of minimum total potential energy and Variational principles.

Singh, Rao, and Iyengar (1991) studied the nonlinear vibration behavior of unsymmetric composite beams. The governing equations were formulated using the classical lamination theory, first-order shear deformation theory and higher-order shear deformation theory. The analysis was performed using elements having 8,10 or 12 degrees of freedom per node. The behavior of isotropic and symmetric orthotropic laminates was studied. The equations were developed using Von Karman large deflection theory to analyze large amplitude free vibration. The equations were solved using direct numerical integration for various boundary conditions, lay-up sequences and slenderness ratios.

Kapania and Raciti (1989a) developed a finite element to study the nonlinear vibrations of unsymmetric laminated beams. A beam element with twenty degrees of freedom was considered for the analysis. The displacement functions were interpolated through Hermite polynomials. The governing equations were derived using Lagrange equations of motion. The effect of shear deformation was considered for linear vibrations 
and neglected for nonlinear vibrations. The formulation was also used to predict the static response, free vibration and nonlinear vibration of isotropic and laminated beams. The inplane boundary conditions were found to affect the nonlinear response of the beam. The large deflection theory neglecting shear effects produced reasonably accurate results for the nonlinear vibrations of thin plates.

Shi, Lam and Tay (1998) studied the effect of the bending strain on the accuracy of the finite element model. A higher-order shear deformation theory was used to formulate the model. Different strain expressions were derived for the same higher-order shear deformation theory. These strain expressions altered the interpolation order of the element bending strain. Similar beam elements having the same number of nodes and the same number of degrees of freedom but different strain expressions resulted in different accuracy levels of the result.

Chen and Yang (1985) developed a finite element with twelve degrees of freedom for symmetrically laminated beams. The effect of shear deformation was considered while formulating the equations. A homogeneous anisotropic beam theory was used for formulation. This theory accounted for coupling between bending and torsion. A simple and efficient procedure was developed to solve the equations. This procedure was programmed using the Basic Computer language. The program was capable of performing stress and vibration analyses. The behaviors of isotropic and orthotropic laminated beams were studied. The results were compared with those available in literature.

Marur and Kant (1998) developed a finite element model for the analysis of laminated composite and sandwich beams. A Higher-order theory was used for the 
formulation and the results were compared with that of the first-order theory. The higherorder theory does not use any shear correction factor. In the higher-order theory, every layer was considered to be in a state of plane stress. Hamilton's principle was used to generate the governing equations. Scaling all the diagonal elements of the consistent mass matrix generated a diagonal mass matrix. Then the equations were solved using the central difference method. It was observed that the higher-order model was more effective to study the behavior of both composite and sandwich beams compared to the first-order theory.

Kapania and Singhvi (1991) studied the behavior of laminated tapered skew plates and developed a method to study their free vibration characteristics. The governing equation was formulated using the Rayleigh-Ritz method. Chebyshev polynomials were used to describe the displacement distribution. The Gaussian Quadrature was used to evaluate the integrals. The analysis was performed on isotropic, orthotropic, symmetrically laminated and unsymmetrically laminated composite plates. The results were compared with those available in literature.

Kapania and Raciti (1989b) summarized the developments in the analysis of composite beams and plates. Analytical and numerical methods used in the procedures were discussed. Analytical methods such as the Galerkin method and Rayleigh-Ritz method were used to derive the governing equations. The results from linear and nonlinear vibration analysis of symmetrical and unsymmetrical plates were presented. The finite element method was used for solving the governing equations. The effect of transverse shear deformation on the behavior of the beam was presented. It was found that the effect of shear deformation decreased with an increase in amplitude and that the 
rotary inertia effect was small compared to the shear effect. Transient response of composite beams was also found.

Lee and Lee (1990) studied the behavior of a composite plate wing. The equations were formulated based on generalized Mindlin's Theory. Shear correction coefficients were used. The influence of sweep angle, fiber orientation, aspect ratio and taper ratio of a composite wing on the vibration characteristics was studied using the finite element method. An eight-noded quadrilateral element produced accurate results. The natural frequencies and the mode shapes were found to be largely affected by the aspect ratio and fiber orientation.

Chandrasekaran (2000) studied the behavior of moving beams made of laminated composites. The governing equations were derived based on the variational principle. The formulation was done for both the classical laminate plate theory and the first-order shear deformation theory. The boundary conditions were introduced via Lagrange multipliers. The finite element equations were then solved using Newmark's implicit method. The displacement response of the beam was studied for symmetric and unsymmetric laminates.

Kadivar and Mohebpour (1997 and 1998) studied the dynamic behavior of laminated composite beams under the action of moving loads. Analysis was performed for symmetric cross ply and un-symmetric angle ply laminates. The governing equations were formulated based on Hamilton's principle. The equations were formulated for three deformation theories namely the classical laminate plate theory, first-order sheardeformation theory and higher-order shear-deformation theory. A beam element with 
twenty-four degrees of freedom based on Hermite interpolation polynomials was used. The equations were solved using Newmark's method.

\subsubsection{Mixed Models}

Sheng and Ye (2002) developed a semi analytical finite element model for the stress analysis of cross-ply laminated composite plates. The model is based on a mixed variational principle that includes variation of both displacements and stresses. The differential equation was also known as state equation. It was derived using a recursive formulation. This recursive formulation leads to the solution of a system of algebraic equations whose order does not depend on number of layers. An iso-parametric element was used to describe stress distribution and displacement distribution. Numerical tests were performed and the results were compared with three-dimensional analytical solutions.

Desai and Ramtekkar (2002) formulated a mixed finite element model to analyze laminated composite beams. The fundamental elastic equations were used to invoke transverse stress as a nodal degree of freedom. Thus the continuity of transverse stress and displacement fields through the thickness of the laminated beam was accounted for. A six-noded element with four degrees of freedom at each node was used for the analysis.

\subsection{Need for present research}

The use of composite materials for engineering structures replacing conventional materials has increased steadily due to better engineering properties. The ability of the composite material to incorporate material design in the design process of engineering 
structures makes it a preferable alternative to conventional materials. There has been extensive research in the field of composite materials in the recent years. As stated earlier composites are widely used in the fields of robotics, structural and earthquake engineering. Due to these reasons it is important to study the behavior of composite beams. Chandrasekaran (2000) have studied the behavior of a composite moving beam based on CLPT and FSDT. The CLPT is inadequate since it does not account for transverse shear components. The FSDT uses a shear correction factor, which is only an approximation. Therefore, a higher-order theory that could provide a better solution can be used to formulate the finite element model. In this thesis an attempt is made to predict the dynamic behavior of a moving beam using HSDT that overcomes the disadvantages of CLPT and FSDT theories.

\subsection{Objectives}

The Objectives of the thesis are:

- To formulate a higher-order finite element model for a composite moving beam and analyze its dynamic behavior. The formulation would be based on a higherorder shear deformation theory and the variational method. The essential conditions are to be applied via Lagrange multipliers.

- To calculate the fundamental frequencies and the time-dependent deflections using time-integration methods such as Newmark's method Wilson theta method.

- To generate a MATLAB code to solve the finite element equations for the composite moving beam with different lay-up configurations. The beam is 
assumed to make a sinusoidal horizontal motion with the specified amplitude and frequency.

\subsection{Thesis Overview}

Chapter two deals with the beam lay-up configuration, introduction to different plate theories and their displacement distributions, formulation of the governing equations using variational principle and energy considerations.

Chapter three details the formulation of the finite element formulation of the stiffness and inertia matrices, Lagrange multiplier approach, Gaussian integration procedure and the time integration schemes used for solving the governing equations.

Chapter four presents the results in the form of the dynamic response of a composite moving beam formulated using higher-order shear deformation theory.

Chapter five contains the conclusions of the present work and recommendations for future work. 


\section{THEORETICAL FORMULATION}

\subsection{Introduction}

In this chapter the parameters and coordinate systems characterizing a moving beam are defined. Composite materials are designed to obtain better engineering properties. For this reason composite materials are used in a wide variety of applications and are preferred over conventional materials in many instances. Some of the attractive properties of composite materials are: strength to weight ratio, resistance to corrosion and fatigue life. In various applications, composite beams replace beams made of conventional materials. Composite beams are used in structural members where they are subjected to axial, transverse and torsional loading. There are several plate theories available for the analysis of composite plates. The process of adapting some of these theories to the case of a beam is the main thrust of this chapter.

\subsection{Moving Beam Definition}

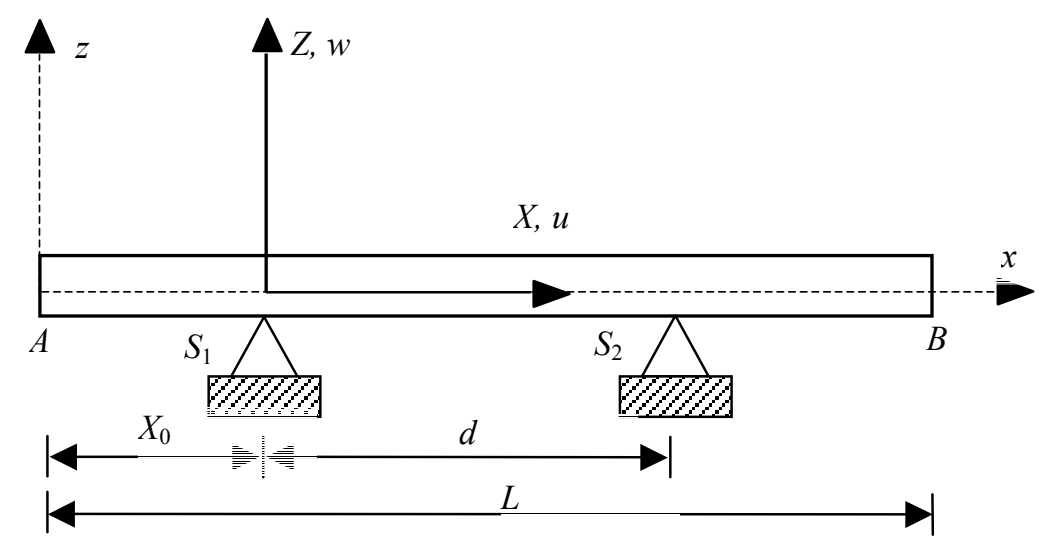

Figure 2.1 Coordinate systems for the moving beam 
A beam moving axially relative to two fixed supports is described in this section. Figure 2.1 shows a beam $A B$ of length $L$ resting on two fixed supports $S_{1}$ and $S_{2}$ separated by a distance $d$. An inertial reference frame $(X, Z)$ is considered with its origin at $S_{1}$ and the $X$-axis along the length of the beam. The beam has a rigid body motion relative to the supports in the longitudinal $(X)$ direction. This horizontal motion of the beam as a function of time $t$ can be described by $X_{A}(t)$ and it is always negative. The initial distance between the left end $A$ of the beam and the first support $S_{1}$ is denoted as $X_{0}$. The beam is capable of deforming in the longitudinal $(u)$ and transverse $(w)$ directions. A moving frame $(x, z)$ is attached to the left end of the beam. This frame is considered to move longitudinally in phase with the rigid-body motion of the beam. The transformation between the inertial and the moving frames is given by

$$
\begin{aligned}
& x(t)=X(t)-X_{A}(t) \\
& z(t)=Z(t)
\end{aligned}
$$

This moving frame is used for the formulation of the finite element equations and in this frame the supports move relative to the left end of the beam.

\subsubsection{Lay-up Configuration}

Figure 2.2 displays the naming convention and the lamina stacking sequence of a composite laminate. The laminate has $n$ layers and a total height of $h$, which is equal to the sum of the thicknesses of all layers. The lateral coordinates are measured from a reference plane located at the mid-surface of the composite. The quantity $z_{k}$ represents the $z$-coordinate of the top of the $k^{\text {th }}$ layer from the reference plane. The quantity $\overline{z_{k}}$ represents the $z$-coordinate of the middle surface of the $k^{\text {th }}$ layer. The thickness of the $k^{t h}$ 
layer is $t_{k}$. In the case a of symmetric laminate the layers about the reference plane form a mirror image.

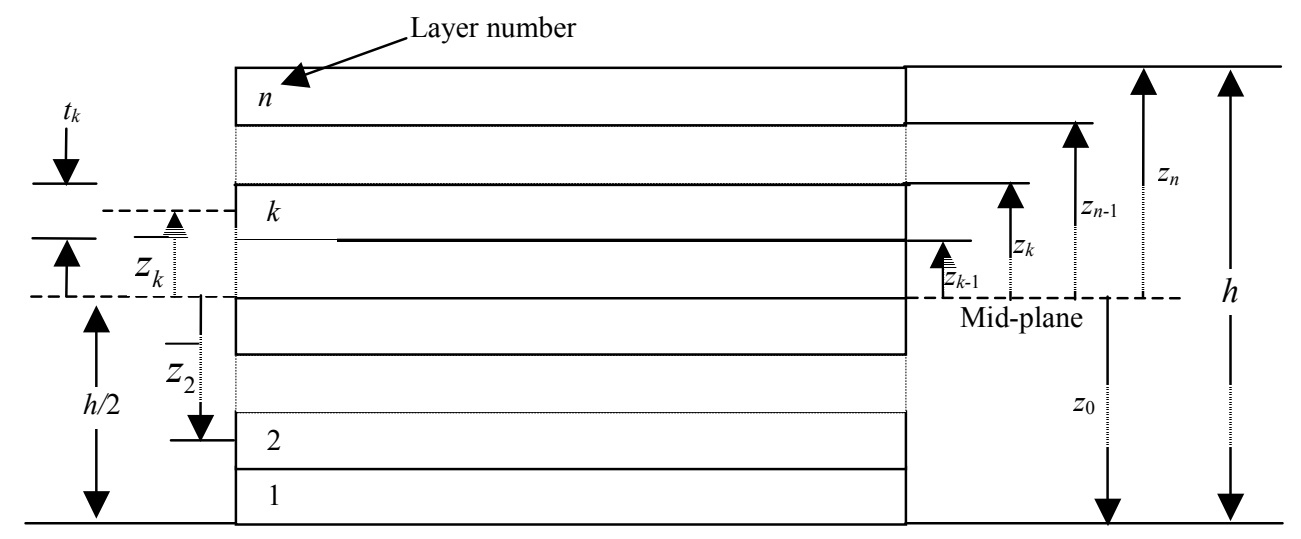

Figure 2.2 Composite lay-up configuration

\subsubsection{Force and Moment Resultants}
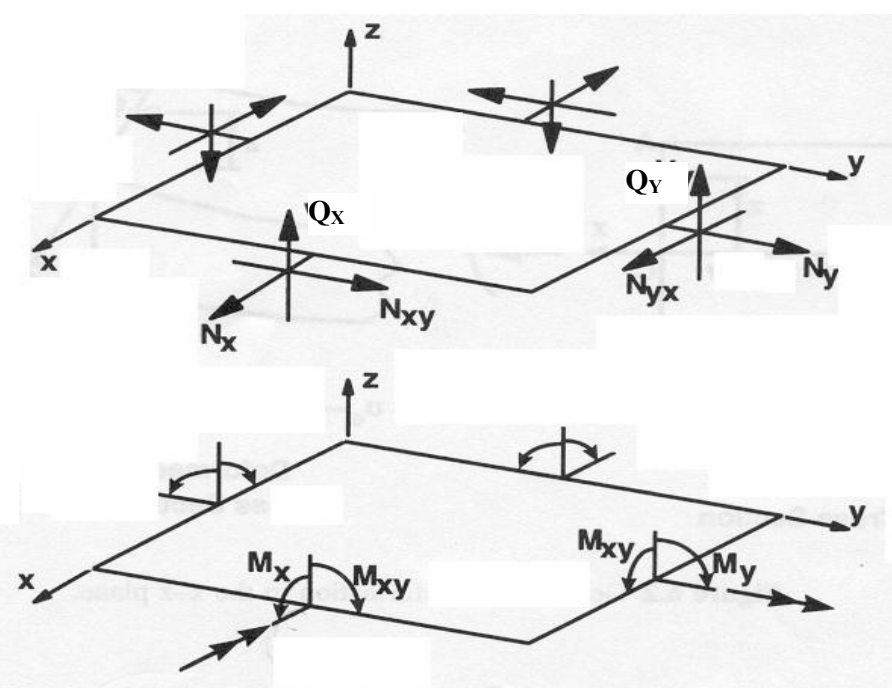

Figure 2.3 Force and Moment Resultants on a flat plate [Barbero (1998)] 
Figure 2.3 shows the positive directions for force and moment resultants. The inplane force resultants acting along the $x$ and $y$ directions are represented by $N_{x}, N_{y}$, and $N_{x y}$. The moment resultants are represented by $M_{x}, M_{y}$, and $M_{x y}$ and the transverse shear force resultants by $Q_{x}$ and $Q_{y}$.

\subsection{Beam Motion}

The longitudinal rigid-body motion of the beam is similar to that assumed by Sreeram and Sivaneri (1997) and Chandrasekaran (2000). It is defined as

$$
X_{A}(t)=-X_{0}+A \operatorname{Sin}(\Omega t)
$$

where $A$ is the amplitude of motion, $X_{0}$, the initial distance between the left end of the beam and the first support $S_{1}$, and $\Omega$ the frequency of axial motion. The velocity $\left(V_{B}{ }^{L}\right)$

and acceleration $\left(a_{B}{ }^{L}\right)$ of the longitudinal rigid-body motion of the beam are obtained by differentiating $X_{A}(t)$ with respect to $t$.

$$
\begin{aligned}
& V_{B}^{L}=A \Omega \operatorname{Cos}(\Omega t) \\
& a_{B}^{L}=-A \Omega^{2} \operatorname{Sin}(\Omega t)
\end{aligned}
$$

In the moving coordinates, the motion of the supports are given by

$$
\begin{aligned}
& x_{S 1}=X_{0}-A \operatorname{Sin}(\Omega t) \\
& x_{S 2}=X_{0}-A \operatorname{Sin}(\Omega t)+d
\end{aligned}
$$

\subsection{Plate Theories}

In the present research, a higher-order shear-deformation theory is used for the analysis of a moving beam. Composite laminates usually have larger planar dimensions 
compared to their thickness. Therefore they are treated as plate elements. The kinematic behavior of the plate elements are described by plate theories. The most commonly used plate theories are the Classical Laminate Plate Theory (CLPT) and First-order Shear Deformation Theory (FSDT). The Classical laminate plate theory for composite laminates is an extension of the classical plate theory of isotropic materials. Kirchoff's hypotheses are used in the derivation of the plate stiffness and compliance equations. The assumptions, as stated by Reddy (1997), for CLPT are:

1. Straight lines perpendicular to the mid-surface (transverse normals) before deformation remain straight after deformation.

2. The transverse normals do not experience elongation. $\left(\varepsilon_{z z}=0\right)$

3. The transverse normals rotate such that they remain perpendicular to the midsurface after deformation. $\left(\varepsilon_{x z}=0\right.$ and $\left.\varepsilon_{y z}=0\right)$

In addition to Kirchoff's hypothesis, the following assumptions are also used:

4. The layers are perfectly bonded together.

5. The material of each layer is linearly elastic and has two planes of material symmetry (i.e., orthotropic).

6. Each layer is of uniform thickness.

7. The strains and displacements are small.

8. The transverse shear stresses on the top and bottom surfaces of the laminate are zero.

Composites have very low transverse shear modulus compared to their on-axis modulus. In the case of CLPT, the effects of transverse shear are neglected since transverse shear strains $\left(\gamma_{x z}\right.$ and $\left.\gamma_{y z}\right)$ are assumed to be zero. This may make CLPT 
inadequate for the analysis of the dynamic response even for a beam with a high slenderness ratio. To consider the effect of transverse shear, an FSDT can be used. The FSDT uses the same assumptions as in CLPT except for the third Kirchoff's hypothesis. In FSDT the transverse normal is assumed to be straight but not perpendicular to the midsurface after deformation and therefore transverse shear strains are not zero. The FSDT uses a shear correction factor, which is only an approximation. To avoid the shear correction factor and to represent the kinematics better than FSDT, higher-order shear deformation theories can be used. The third order theory is also based on the same assumptions as that of FSDT, except that the assumption on the straightness of the transverse normal after deformation is relaxed. The transverse normal is no longer

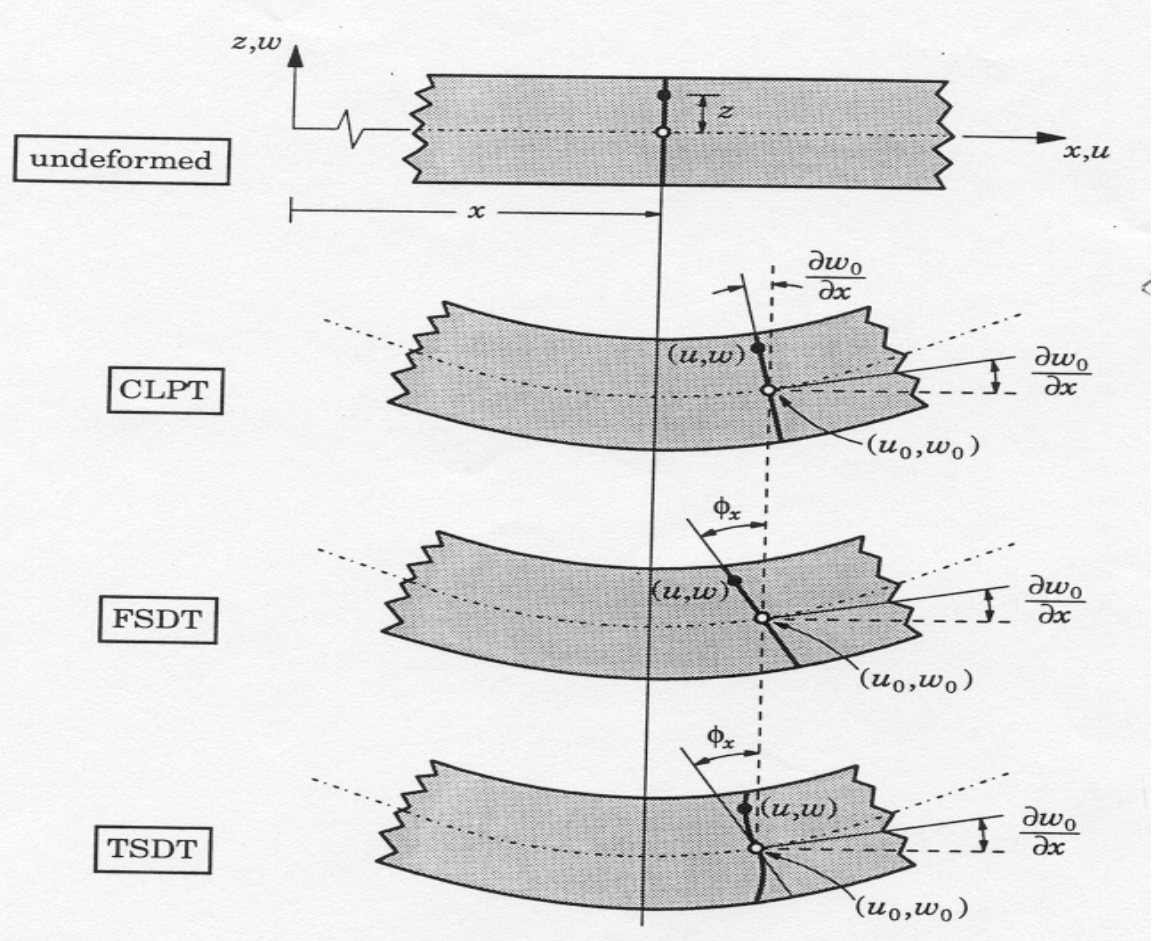

Figure 2.4 Deformation of transverse normal for CLPT, FSDT and HSDT [Reddy (1997)]

inextensible, making the deformations as a function of the thickness coordinate $z$. 
In HSDT, the displacement field $(u, v, w)$ in the $(x, y, z)$ directions, respectively, can be expressed as [Reddy (1997)]:

$$
\begin{aligned}
& u(x, y, z, t)=u_{0}(x, y, t)+z \phi_{x}(x, y, t)-c_{1} z^{3}\left(\phi_{x}+c_{0} \frac{\partial w}{\partial x}\right) \\
& v(x, y, z, t)=v_{0}(x, y, t)+z \phi_{y}(x, y, t)-c_{1} z^{3}\left(\phi_{y}+c_{0} \frac{\partial w}{\partial y}\right) \\
& w(x, y, z, t)=w(x, y, t)=w_{b}(x, y, t)+w_{s}(x, y, t)
\end{aligned}
$$

where $u_{0}$ and $v_{0}$ are the inplane displacements at the midplane and $\phi_{x}$ and $\phi_{\mathrm{y}}$ are the rotations of a transverse normal about the $y$ and $x$ axes, respectively (see Figure 2.4). The bending deformation $w$ consists of a pure bending component, $w_{b}$ and a shear component, $w_{s}$. Note that $w_{b}, w_{s}$, and consequently $w$, are assumed independent of the thickness coordinate $z$. The coefficient $c_{0}$ is assumed to be unity while $c_{1}$ is called a tracer. The displacement field of FSDT can be recovered from Eq. (2.5) by setting $c_{1}=0$. By applying the condition that the transverse shear stresses $\tau_{y z}$ and $\tau_{x z}$ vanish at the top and bottom surfaces of the laminate $\left(z= \pm \frac{h}{2}\right)$, the valueof $c_{1}$ for HSDT is calculated as $\frac{4}{3 h^{2}}$.

Figure 2.4 shows the assumed deformation of a transverse normal for CLPT, FSDT, and HSDT.

\subsection{Hamilton's Principle}

The governing equations of the problem is derived from Hamilton's principle, which is written as,

$$
\Delta_{p}=\int_{t_{1}}^{t_{2}}(\delta U-\delta T-\delta W) d t=0
$$


where $\delta U$ is the virtual strain energy, $\delta T$ is the virtual kinetic energy, and $\delta W$ is the virtual workdone.

\subsubsection{Kinematics Equations of a composite plate}

The nonlinear kinematic equations for moderate rotations are given by [Reddy (1997)],

$\varepsilon_{x}=\frac{\partial u}{\partial x}+\frac{1}{2}\left(\frac{\partial w}{\partial x}\right)^{2}$

$\varepsilon_{y}=\frac{\partial v}{\partial y}+\frac{1}{2}\left(\frac{\partial w}{\partial y}\right)^{2}$

$\gamma_{x y}=\left(\frac{\partial u}{\partial y}+\frac{\partial v}{\partial x}+\frac{\partial w}{\partial x} \frac{\partial w}{\partial y}\right)$

$\gamma_{y z}=\left(\frac{\partial v}{\partial z}+\frac{\partial w}{\partial y}\right)$

$\gamma_{x z}=\left(\frac{\partial u}{\partial z}+\frac{\partial w}{\partial x}\right)$

Substituting Eq. (2.5) into Eq. (2.7) we get,

$$
\begin{aligned}
& \varepsilon_{x}=\frac{\partial u_{0}}{\partial x}+\frac{1}{2}\left(\frac{\partial w}{\partial x}\right)^{2}+z \frac{\partial \phi_{x}}{\partial x}-c_{1} z^{3}\left(\frac{\partial \phi_{x}}{\partial x}+\frac{\partial^{2} w}{\partial x^{2}}\right) \\
& \varepsilon_{y}=\frac{\partial v_{0}}{\partial y}+\frac{1}{2}\left(\frac{\partial w}{\partial y}\right)^{2}+z \frac{\partial \phi_{y}}{\partial y}-c_{1} z^{3}\left(\frac{\partial \phi_{y}}{\partial y}+\frac{\partial^{2} w}{\partial y^{2}}\right) \\
& \gamma_{x y}=\frac{\partial u_{0}}{\partial y}+\frac{\partial v_{0}}{\partial x}+\frac{\partial w}{\partial x} \frac{\partial w}{\partial y}+z\left(\frac{\partial \phi_{x}}{\partial y}+\frac{\partial \phi_{y}}{\partial x}\right)-c_{1} z^{3}\left(\frac{\partial \phi_{x}}{\partial y}+\frac{\partial \phi_{y}}{\partial x}+2 \frac{\partial^{2} w}{\partial x \partial y}\right) \\
& \gamma_{y z}=\phi_{y}+\frac{\partial w}{\partial y}-3 c_{1} z^{2}\left(\phi_{y}+\frac{\partial w}{\partial y}\right)
\end{aligned}
$$


$\gamma_{z x}=\phi_{x}+\frac{\partial w}{\partial x}-3 c_{1} z^{2}\left(\phi_{x}+\frac{\partial w}{\partial x}\right)$

From Figure 2.4, the transverse normal rotations at the midplane, $\phi_{x}$ and $\phi_{\mathrm{y}}$ can be written as,

$$
\begin{gathered}
\phi_{x}=\frac{\partial u_{0}}{\partial z}=-\frac{\partial w_{b}}{\partial x} \\
\phi_{y}=\frac{\partial v_{0}}{\partial z}=-\frac{\partial w_{b}}{\partial y}
\end{gathered}
$$

Then $\phi_{x}+\frac{\partial w}{\partial x}=-\frac{\partial w_{b}}{\partial x}+\frac{\partial w_{b}}{\partial x}+\frac{\partial w_{s}}{\partial x}=\frac{\partial w_{s}}{\partial x}$

Similarly, $\phi_{y}+\frac{\partial w}{\partial y}=\frac{\partial w_{s}}{\partial y}$

The strains of Eq. (2.8) can also be written as,

$$
\begin{aligned}
& \varepsilon_{x}=\varepsilon_{x}^{(0)}+z \varepsilon_{x}^{(1)}+z^{3} \varepsilon_{x}^{(3)} \\
& \varepsilon_{y}=\varepsilon_{y}^{(0)}+z \varepsilon_{y}^{(1)}+z^{3} \varepsilon_{y}^{(3)} \\
& \gamma_{x y}=\gamma_{x y}^{(0)}+z \gamma_{x y}^{(1)}+z^{3} \gamma_{x y}^{(3)} \\
& \gamma_{y z}=\gamma_{y z}^{(0)}+z^{2} \gamma_{y z}^{(2)} \\
& \gamma_{z x}=\gamma_{z x}^{(0)}+z^{2} \gamma_{z x}^{(2)}
\end{aligned}
$$

where,

$$
\begin{aligned}
& \varepsilon_{x}^{(0)}=\frac{\partial u_{0}}{\partial x}+\frac{1}{2}\left(\frac{\partial w_{b}}{\partial x}\right)^{2}+\frac{1}{2}\left(\frac{\partial w_{s}}{\partial x}\right)^{2} \\
& \varepsilon_{x}^{(1)}=-\frac{\partial^{2} w_{b}}{\partial x^{2}}
\end{aligned}
$$




$$
\begin{aligned}
& \varepsilon_{x}^{(3)}=-c_{1} \frac{\partial^{2} w_{s}}{\partial x^{2}} \\
& \varepsilon_{y}^{(0)}=\frac{\partial v_{0}}{\partial x}+\frac{1}{2}\left(\frac{\partial w_{b}}{\partial y}\right)^{2}+\frac{1}{2}\left(\frac{\partial w_{s}}{\partial y}\right)^{2} \\
& \varepsilon_{y}^{(1)}=-\frac{\partial^{2} w_{b}}{\partial y^{2}} \\
& \varepsilon_{y}^{(3)}=-c_{1} \frac{\partial^{2} w_{s}}{\partial y^{2}} \\
& \gamma_{x y}^{(0)}=\frac{\partial u_{0}}{\partial y}+\frac{\partial v_{0}}{\partial x}+\left(\frac{\partial w_{b}}{\partial x}+\frac{\partial w_{s}}{\partial x}\right)\left(\frac{\partial w_{b}}{\partial y}+\frac{\partial w_{s}}{\partial y}\right)
\end{aligned}
$$

The term $\left(\frac{\partial w_{b}}{\partial x}+\frac{\partial w_{s}}{\partial x}\right)\left(\frac{\partial w_{b}}{\partial y}+\frac{\partial w_{s}}{\partial y}\right)$ in the above expression is neglected in further considerations to avoid nonlinearity in the governing equations.

$$
\begin{aligned}
& \gamma_{x y}^{(1)}=-2 \frac{\partial^{2} w_{b}}{\partial x \partial y} \\
& \gamma_{x y}^{(3)}=-2 c_{1} \frac{\partial^{2} w_{s}}{\partial x \partial y} \\
& \gamma_{y z}^{(0)}=\frac{\partial w_{s}}{\partial y} \\
& \gamma_{y z}^{(2)}=-3 c_{1} \frac{\partial w_{s}}{\partial y} \\
& \gamma_{x z}^{(0)}=\frac{\partial w_{s}}{\partial x} \\
& \gamma_{x z}^{(2)}=-3 c_{1} \frac{\partial w_{s}}{\partial x}
\end{aligned}
$$




\subsubsection{Virtual Strain Energy for a Plate}

The total virtual strain energy of a plate is given by,

$$
\delta U=\iiint_{V}\left[\sigma_{x} \delta \varepsilon_{x}+\sigma_{y} \delta \varepsilon_{y}+\tau_{x y} \delta \gamma_{x y}+\tau_{y z} \delta \gamma_{y z}+\tau_{x z} \delta \gamma_{x z}\right] d V
$$

where $\delta \varepsilon_{x}, \delta \varepsilon_{y}, \delta \gamma_{x y}, \delta \gamma_{y z}$, and $\delta \gamma_{x z}$ are the virtual strains and $V$ is the volume of the plate. Separating the volume integral into an integral over the thickness coordinate $z$ and an area integral in the $x, y$ directions we get,

$$
\delta U=\iint_{A} \int_{-\frac{h}{2}}^{\frac{h}{2}}\left[\sigma_{x} \delta \varepsilon_{x}+\sigma_{y} \delta \varepsilon_{y}+\tau_{x y} \delta \gamma_{x y}+\tau_{y z} \delta \gamma_{y z}+\tau_{x z} \delta \gamma_{x z}\right] d A d z
$$

Define the stress resultants as follows,

$$
\begin{aligned}
& \left(N_{x}, N_{y}, N_{x y}\right)=\int_{-\frac{h}{2}}^{-\frac{h}{2}}\left(\sigma_{x}, \sigma_{y}, \tau_{x y}\right) d z \\
& \left(M_{x}, M_{y}, M_{x y}\right)=\int_{-\frac{h}{2}}^{-\frac{h}{2}}\left(\sigma_{x}, \sigma_{y}, \tau_{x y}\right) z d z \\
& \left(P_{x}, P_{y}, P_{x y}\right)=\int_{-\frac{h}{2}}^{-\frac{h}{2}}\left(\sigma_{x}, \sigma_{y}, \tau_{x y}\right) z^{3} d z \\
& \left(Q_{x}, Q_{y}\right)=\int_{-\frac{h}{2}}^{-\frac{h}{2}}\left(\tau_{x z}, \tau_{y z}\right) d z \\
& \left(R_{x}, R_{y}\right)=\int_{-\frac{h}{2}}^{-\frac{h}{2}}\left(\tau_{x z}, \tau_{y z}\right) z^{2} d z
\end{aligned}
$$

The quantities $\left(N_{x}, N_{y}, N_{x y}\right)$ are the inplane force resultants, $\left(M_{x}, M_{y}, M_{x y}\right)$ are the moment resultants, $\left(Q_{x}, Q_{y}\right)$ are the transverse force resultants and $\left(P_{x}, P_{y}, P_{x y}, R_{x}, R_{y}\right)$ are higherorder stress resultants. Then the strain energy equation of the beam reduces to: 
$\delta U=\iint_{A}\left[\begin{array}{l}N_{x} \delta \varepsilon_{x}^{(0)}+M_{x} \delta \varepsilon_{x}^{(1)}+P_{x} \delta \varepsilon_{x}^{(3)}+N_{y} \delta \varepsilon_{y}^{(0)}+M_{y} \delta \varepsilon_{y}^{(1)}+P_{y} \delta \varepsilon_{y}^{(3)}+N_{x y} \delta \gamma_{x y}^{(0)} \\ +M_{x y} \delta \gamma_{x y}^{(1)}+P_{x y} \delta \gamma_{x y}^{(3)}+Q_{x} \delta \gamma_{z x}^{(0)}+R_{x} \delta \gamma_{z x}^{(2)}+Q_{y} \delta \gamma_{y z}^{(0)}+R_{y} \delta \gamma_{y z}^{(2)}\end{array}\right] d A$

\subsubsection{Constitutive Equations}

The relation between the stress resultants and strains are given by:

$$
\begin{aligned}
& \left\{\begin{array}{l}
\{N\} \\
\{M\} \\
\{P\}
\end{array}\right\}=\left[\begin{array}{lll}
{[A]} & {[B]} & {[E]} \\
{[B]} & {[D]} & {[F]} \\
{[E]} & {[F]} & {[H]}
\end{array}\right]\left\{\begin{array}{l}
\left\{\varepsilon^{(0)}\right\} \\
\left\{\varepsilon^{(1)}\right\} \\
\left\{\varepsilon^{(3)}\right\}
\end{array}\right\} \\
& \left\{\begin{array}{l}
\{Q\} \\
\{R\}
\end{array}\right\}=\left[\begin{array}{ll}
{[A]} & {[D]} \\
{[D]} & {[F]}
\end{array}\right]\left\{\begin{array}{l}
\left\{\gamma^{(0)}\right\} \\
\left\{\gamma^{(2)}\right\}
\end{array}\right\}
\end{aligned}
$$

Vectors $\{N\}$ and $\{M\}$ denote the force and moment resultants. Vector $\{Q\}$ represents the transverse force resultants, while vectors $\{P\}$ and $\{R\}$ denote the higher-order stress resultants. Matrices $[A],[B]$ and $[D]$ contain the extension stiffness, bending-extension coupling and bending stiffness coefficients while matrices $[E],[F]$ and $[H]$ have higherorder stiffness coefficient terms. The coefficient matrices in Eq. (2.17) are obtained from,

$$
\left(A_{i j}, B_{i j}, D_{i j}, E_{i j}, F_{i j}, H_{i j}\right)=\sum_{k=1}^{n} \int_{z_{k-1}}^{z_{k}} \bar{Q}_{i j}^{(k)}\left(1, z, z^{2}, z^{3}, z^{4}, z^{6}\right) d z
$$

The square matrices in Eq. (2.17) are of the order $3 \times 3$ and the stiffness coefficients are defined for $i, j=1,2,6$. The $\bar{Q}_{i j}^{(k)}$ represent the off-axis material stiffness coefficients of the $k^{\text {th }}$ layer. Matrices in Eq. (2.18) are obtained from,

$$
\left(A_{i j}, D_{i j}, F_{i j}\right)=\sum_{k=1}^{n} \int_{z_{k-1}}^{z_{k}} \bar{Q}_{i j}^{(k)}\left(1, z^{2}, z^{6}\right) d z
$$

where $[A],[D]$ and $[F]$ are $2 \times 2$ matrices with $i, j=4,5$. 


\subsubsection{Reduction of Plate Equations to Beam}

For isotropic materials beam theories are developed first since they are much simpler than the corresponding plate theories. On the other hand plate theories are developed first in the case of composite materials. There must be a systematic way of reducing these plate theories into corresponding beam theories. This process for HSDT is outlined in this section.

For beams the lateral resultant forces are negligible. Therefore, $N_{y}, M_{y}, P_{y}$ are set to zero in Eq. (2.17). Similarly $Q_{y}$ and $R_{y}$ are set to zero in Eq. (2.18). Rearranging and partitioning Eq. (2.17) we get,

$$
\left\{\begin{array}{l}
N_{x} \\
N_{x y} \\
M_{x} \\
M_{x y} \\
P_{x} \\
P_{x y} \\
\hdashline 0 \\
0 \\
0
\end{array}\right\}=\left[\begin{array}{llllll:lll}
A_{11} & A_{16} & B_{11} & B_{16} & E_{11} & E_{16} & A_{12} & B_{12} & E_{12} \\
A_{16} & A_{66} & B_{16} & B_{66} & E_{16} & E_{66} & A_{26} & B_{26} & E_{26} \\
B_{11} & B_{16} & D_{11} & D_{16} & F_{11} & F_{16} & B_{12} & D_{12} & F_{12} \\
B_{16} & B_{66} & D_{16} & D_{66} & F_{16} & F_{66} & B_{26} & D_{26} & F_{26} \\
E_{11} & E_{16} & F_{11} & F_{16} & H_{11} & H_{16} & E_{12} & F_{12} & H_{12} \\
E_{16} & E_{66} & F_{16} & F_{66} & H_{16} & H_{66} & E_{26} & F_{26} & H_{26} \\
\hdashline A_{12} & A_{26} & B_{12} & B_{26} & E_{12} & E_{26} & A_{22} & B_{22} & E_{22} \\
B_{12} & B_{26} & D_{12} & D_{26} & F_{12} & F_{26} & B_{22} & D_{22} & F_{22} \\
E_{12} & E_{26} & F_{12} & F_{26} & H_{12} & H_{26} & E_{22} & F_{22} & H_{22}
\end{array}\right]\left\{\begin{array}{l}
\varepsilon_{x}^{(0)} \\
\gamma_{x y}^{(0)} \\
\varepsilon_{x}^{(1)} \\
\gamma_{x y}^{(1)} \\
\varepsilon_{x}^{(3)} \\
\gamma_{x y}^{(3)} \\
\varepsilon_{y}^{(0)} \\
\varepsilon_{y}^{(1)} \\
\varepsilon_{y}^{(3)}
\end{array}\right\}
$$

Introducing notations for the partitions we get,

$$
\left\{\begin{array}{c}
\{\bar{N}\} \\
\{0\}
\end{array}\right\}=\left[\begin{array}{ll}
{\left[T^{11}\right]} & {\left[T^{12}\right]} \\
{\left[T^{21}\right]} & {\left[T^{22}\right]}
\end{array}\right]\left\{\begin{array}{c}
\overline{\bar{\varepsilon}}\} \\
\left\{\overline{\varepsilon_{y}}\right\}
\end{array}\right\}
$$

It can be seen that

$$
\left[T^{21}\right]=\left[T^{12}\right]^{T}
$$

Expanding Eq. (2.22) we get, 


$$
\begin{aligned}
& \{\bar{N}\}=\left[T^{11}\right]\{\bar{\varepsilon}\}+\left[T^{12}\right]\left\{\overline{\varepsilon_{y}}\right\} \\
& \{0\}=\left[T^{21}\right]\{\bar{\varepsilon}\}+\left[T^{22}\right]\left\{\overline{\varepsilon_{y}}\right\}
\end{aligned}
$$

Eliminating $\left\{\bar{\varepsilon}_{y}\right\}$ in Eq. (2.24) we get,

$$
\{\bar{N}\}=[T]\{\bar{\varepsilon}\}
$$

where $[T]=\left[\left[T^{11}\right]-\left[T^{12}\right]\left[T^{22}\right]^{-1}\left[T^{21}\right]\right]$

In a similar way Eq. (2.18) can be rearranged by setting $Q_{y}=R_{y}=0$,

$$
\left\{\begin{array}{c}
Q_{x} \\
R_{x} \\
\hdashline 0 \\
0
\end{array}\right\}=\left[\begin{array}{cc:cc}
A_{55} & D_{55} & A_{45} & D_{45} \\
D_{55} & F_{55} & D_{45} & F_{45} \\
\hdashline A_{45} & D_{45} & A_{44} & D_{44} \\
D_{45} & F_{45} & D_{44} & F_{44}
\end{array}\right]\left\{\begin{array}{c}
\gamma_{x z}^{(0)} \\
\gamma_{x z}^{(2)} \\
\hdashline \gamma_{y z}^{(0)} \\
\gamma_{y z}^{(2)}
\end{array}\right\}
$$

Introducing the notations, $w_{s}^{\prime}=\frac{\partial w_{s}}{\partial x}$ and $w_{s}^{y}=\frac{\partial w_{s}}{\partial y}$ in Eq. (2.12), the strain vector in Eq. (2.27) becomes,

$\left\lfloor\begin{array}{llll}\gamma_{x z}^{(0)} & \gamma_{x z}^{(2)} & \gamma_{y z}^{(0)} & \gamma_{y z}^{(2)}\end{array}\right\rfloor=\left\lfloor\begin{array}{lllll}w_{s}^{\prime} & -3 c_{1} w_{s}^{\prime} & w_{s}^{y} & -3 c_{1} w_{s}^{y}\end{array}\right\rfloor$

Define

$$
\begin{aligned}
D_{i j}^{*} & =A_{i j}-6 c_{1} D_{i j}+9 c_{1}^{2} F_{i j} \quad(i, j=4,5) \\
Q_{x}^{*} & =Q_{x}-3 c_{1} R_{x} \\
Q_{y}^{*} & =Q_{y}-3 c_{1} R_{y}
\end{aligned}
$$

Substituting Eq. (2.29) into Eq. (2.27) we get,

$$
\left\{\begin{array}{l}
Q_{y}^{*} \\
Q_{x}^{*}
\end{array}\right\}=K\left[\begin{array}{cc}
D_{44}^{*} & D_{45}^{*} \\
D_{45}^{*} & D_{55}^{*}
\end{array}\right]\left\{\begin{array}{l}
\left\{w_{s}^{y}\right\} \\
\left\{w_{s}^{\prime}\right\}
\end{array}\right\}
$$


A factor $K$ known as the shear correction factor in the above equation is introduced even though HSDT does not require one. This is done to obtain FSDT results from the HSDT formulation by setting $c_{1}=0$ and $K=5 / 6$. For HSDT $K$ will be set to 1 .

For a beam, $Q_{y}^{*}=0$. And thus solving Eq. (2.30) we get,

$Q_{x}^{*}=K D_{55}^{* *} w_{s}^{\prime}$

where $D_{55}^{* *}=D_{55}^{*}-\frac{D_{45}^{* 2}}{D_{44}^{*}}$

\subsubsection{Virtual Strain Energy for a Beam}

As we have seen $N_{y}=M_{y}=P_{y}=Q_{y}=R_{y}=0$ for a beam. Further for a beam of rectangular cross section of width $b$ and length $L$, the double integral in Eq. (2.16) is changed into a line integral along $x$. Thus Eq. (2.16) reduces to,

$\delta U=b \int_{0}^{L}\left[N_{x} \delta \varepsilon_{x}^{(0)}+M_{x} \delta \varepsilon_{x}^{(1)}+P_{x} \delta \varepsilon_{x}^{(3)}+N_{x y} \delta \gamma_{x y}^{(0)}+M_{x y} \delta \gamma_{x y}^{(1)}+P_{x y} \delta \gamma_{x y}^{(3)}+Q_{x}^{*} \delta \gamma_{z x}^{(0)}\right] d x$

Now the $\delta U$ can be written in terms of the deformation quantities. For simpler notation in performaimg this step, define,

$$
\begin{aligned}
& ()^{\prime}=\frac{\partial()}{\partial x} \\
& ()^{y}=\frac{\partial()}{\partial y} \\
& ()^{\prime \prime}=\frac{\partial^{2}()}{\partial x^{2}} \\
& ()^{\prime y}=\frac{\partial^{2}()}{\partial x \partial y}
\end{aligned}
$$




$$
\gamma_{0}=\frac{\partial u_{0}}{\partial y}+\frac{\partial v_{0}}{\partial x}
$$

Then $\delta U$ becomes,

$$
\delta U=b \int_{0}^{L}\left[\begin{array}{l}
N_{x}\left(\delta u_{0}^{\prime}+w_{b}^{\prime} \delta w_{b}^{\prime}+w_{s}^{\prime} \delta w_{s}^{\prime}\right)-M_{x} \delta w_{b}^{\prime \prime}-P_{x} c_{1} \delta w_{s}^{\prime \prime} \\
+N_{x y} \delta \gamma_{0}-2 M_{x y} \delta w_{b}^{\prime y}-2 P_{x y} c_{1} w_{s}^{\prime y}+Q_{x}^{*} w_{s}^{\prime}
\end{array}\right] d x
$$

\subsubsection{Virtual Kinetic Energy for a Beam}

The virtual kinetic energy expression is given by,

$$
\delta T=\iiint_{V} \rho[\dot{u} \delta \dot{u}+\dot{v} \delta \dot{v}+\dot{w} \delta \dot{w}] d V
$$

where $(\bullet)=\frac{\partial()}{\partial t}$. Substituting for the displacements from Eq. (2.5) and making use of Eqs. (2.9) and (2.34) we get,

$$
\delta T=\iiint_{V} \rho\left[\begin{array}{l}
\left(\dot{u}_{0}-z \dot{w}_{b}^{\prime}-c_{1} z^{3} \dot{w}_{s}^{\prime}\right)\left(\delta \dot{u}_{0}-z \delta \dot{w}_{b}^{\prime}-c_{1} z^{3} \delta \dot{w}_{s}^{\prime}\right)+\left(\dot{v}_{0}-z \dot{w}_{b}^{y}-c_{1} z^{3} \dot{w}_{s}^{y}\right) \\
\left(\delta \dot{v}_{0}-z \delta \dot{w}_{b}^{y}-c_{1} z^{3} \delta \dot{w}_{s}^{y}\right)+\left(\dot{w}_{b}+\dot{w}_{s}\right)\left(\delta \dot{w}_{b}+\delta \dot{w}_{s}\right)
\end{array}\right] d V
$$

where $\rho$ is the mass density and ( ) represents partial derivative with respect to time.

The $\dot{v}_{0}$ and $\delta \dot{v}_{0}$ terms are left out for a beam and the expression becomes,

$$
\delta T=\iiint_{V} \rho\left[\begin{array}{l}
\left(\dot{u}_{0}-z \dot{w}_{b}^{\prime}-c_{1} z^{3} \dot{w}_{s}^{\prime}\right) \delta \dot{u}_{0}-\left(z \dot{u}_{0}-z^{2} \dot{w}_{b}^{\prime}-c_{1} z^{4} \dot{w}_{s}^{\prime}\right) \delta \dot{w}_{b}^{\prime}-c_{1}\left(z^{3} \dot{u}_{0}-z^{4} \dot{w}_{b}^{\prime}-c_{1} z^{6} \dot{w}_{s}^{\prime}\right) \delta \dot{w}_{s}^{\prime} \\
+\left(z^{2} \dot{w}_{b}^{y}+c_{1} z^{4} \dot{w}_{s}^{y}\right) \delta \dot{w}_{b}^{y}+c_{1}\left(z^{4} \dot{w}_{b}^{y}+c_{1} z^{6} \dot{w}_{s}^{y}\right) \delta \dot{w}_{s}^{y}+\left(\dot{w}_{b}+\dot{w}_{s}\right) \delta \dot{w}_{b}+\left(\dot{w}_{b}+\dot{w}_{s}\right) \delta \dot{w}_{s}
\end{array}\right] d V
$$

Isolating the $\delta T$ term in Hamilton's principle [Eq. (2.6)] and integrating by parts with respect to $t$, and grouping all time boundary terms and denoting them as $(\ldots)_{t_{1}}^{t_{2}}$, we get, 


$$
\begin{aligned}
& -\int_{t_{1}}^{t_{2}} \delta T d t \\
& =\iiint_{V}\left\{\begin{array}{l}
\int_{t_{2}}^{t_{1}} \rho\left[\begin{array}{l}
\left(\ddot{u}_{0}-z \ddot{w}_{b}^{\prime}-c_{1} z^{3} \ddot{w}_{s}^{\prime}\right) \delta u-\left(z \ddot{u}_{0}-z^{2} \dot{w}_{b}^{\prime}-c_{1} z^{4} \dot{w}_{s}^{\prime}\right) \delta w_{b}^{\prime}-c_{1}\left(z^{3} \ddot{u}_{0}-z^{4} \ddot{w}_{b}^{\prime}-c_{1} z^{6} \ddot{w}_{s}^{\prime}\right) \delta w_{s}^{\prime} \\
+\left(z^{2} \ddot{w}_{b}^{y}+c_{1} z^{4} \ddot{w}_{s}^{y}\right) \delta w_{b}^{y}+c_{1}\left(z^{4} \ddot{w}_{b}^{y}+c_{1} z^{6} \ddot{w}_{s}^{y}\right) \delta w_{s}^{y}+\left(\ddot{w}_{b}+\ddot{w}_{s}\right) \delta w_{b}+\left(\ddot{w}_{b}+\ddot{w}_{s}\right) \delta w_{s}
\end{array}\right] d t \\
+(\cdots)_{t_{1}}^{t_{2}}
\end{array}\right\} d V
\end{aligned}
$$

The time boundary terms do not contribute to the inertia matrix, therefore they can be left out of the $\delta T$ equation. Since the variational quantities no longer contain time derivatives they can be pulled out of the time integral. The volume integral can be split into integrals over thickness, length and width.

$$
-\delta T=b \int_{0}^{L} d x \int_{-\frac{h}{2}}^{\frac{h}{2}} \rho\left[\begin{array}{l}
\left(\ddot{u}_{0}-z \ddot{w}_{b}^{\prime}-c_{1} z^{3} \ddot{w}_{s}^{\prime}\right) \delta u-\left(z \ddot{u}_{0}-z^{2} \dot{w}_{b}^{\prime}-c_{1} z^{4} \dot{w}_{s}^{\prime}\right) \delta w_{b}^{\prime}-c_{1}\left(z^{3} \ddot{u}_{0}-z^{4} \ddot{w}_{b}^{\prime}-c_{1} z^{6} \ddot{w}_{s}^{\prime}\right) \delta w_{s}^{\prime} \\
+\left(z^{2} \ddot{w}_{b}^{y}+c_{1} z^{4} \ddot{w}_{s}^{y}\right) \delta w_{b}^{y}+c_{1}\left(z^{4} \ddot{w}_{b}^{y}+c_{1} z^{6} \ddot{w}_{s}^{y}\right) \delta w_{s}^{y}+\left(\ddot{w}_{b}+\ddot{w}_{s}\right) \delta w_{b}+\left(\ddot{w}_{b}+\ddot{w}_{s}\right) \delta w_{s}
\end{array}\right] d z
$$

The thickness integral can be further reduced using,

$$
I_{i}=\int_{-h / 2}^{h / 2} \rho(z)^{i} d z \quad(\mathrm{I}=0,1, \ldots 6)
$$

where the $I_{i}$ represent inertia coefficients. The $I_{0}, I_{1}$ and $I_{2}$ are the normal, coupled normal-rotary, and rotary inertia coefficients; and $I_{3}, I_{4}$ and $I_{6}$ are higher-order inertia coefficients. 


\section{FINITE ELEMENT MODELING}

\subsection{Introduction}

The finite element method is a numerical procedure widely used in solving engineering problems. Engineering systems can be described by mathematical models, which can be analyzed and solved using finite element methods. In this procedure, the system to be analyzed is first discretized. In solid-mechanics applications the straindisplacement and the stress-strain relations are established. One method of formulating the finite element equations is based on energy considerations and the variational method. Energy methods are very powerful in modeling engineering problems. According to the principle of conservation of energy, the total energy of a conservative system is constant. This principle is one tool in formulating the finite element model. In variational method, vector quantities such as force, displacements, accelerations, etc., are not considered; instead scalar quantities such as work, energy are considered. This method is relatively straight forward and it leads to both the governing equations and the boundary conditions directly.

\subsection{Displacement Distribution}

The composite beam is discretized into nodes and elements. In the present analysis, a higher-order element with three internal nodes and two end nodes is used. The beam is divided into four elements. The number of elements and the number of nodes are based on the results of Chandrasekaran (2000). Lagrangian and Hermitian interpolation functions are used to derive the shape functions. Lagrangian interpolation functions are

used for certain degrees of freedom to ensure $C^{0}$ continuity while Hermitian interpolation 
functions are used for other degrees of freedom to ensure $C^{1}$ continuity. Slope continuity is automatically assured at the internal nodes and therefore they do not have slope degrees of freedom. The end nodes have both displacement degrees of freedom and slope degrees of freedom for the variables with $C^{1}$ continuity.

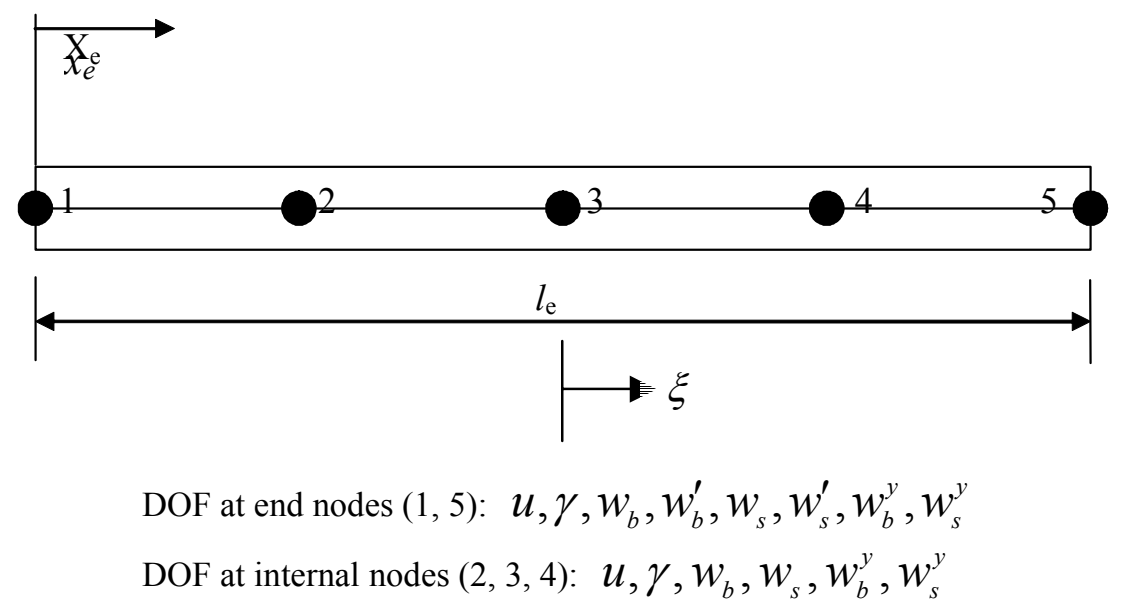

Figure 3.1 Element definition for formulation using HSDT

Figure 3.1 shows a composite beam element of length $l_{e}$ with 2 end nodes and 3 internal nodes. The independent variable is $x_{e}$ measured from the left end of the beam. The dependent variables are:

$$
\begin{aligned}
& u=\text { axial deformation at the midplane } \\
& w_{b}=\text { transverse bending deformation } \\
& w_{\mathrm{s}}=\text { transverse shear deformation } \\
& \gamma=\left(\frac{\partial u}{\partial y}+\frac{\partial v}{\partial x}\right)_{z=0}=\text { mid-plane shear strain } \\
& w_{b}^{y}=\frac{\partial w_{b}}{\partial y}=\text { twist angle associated with bending deformation }
\end{aligned}
$$




$$
w_{s}^{y}=\frac{\partial w_{s}}{\partial y}=\text { twisting angle associated with shear deformation }
$$

At the end nodes, slope continuity $\left(C^{1}\right)$ is maintained for the variables $w_{b}$ and $w_{s}$. The other variables, namely $u, \gamma, w_{b}^{y}, w_{s}^{y}$ obey only $C^{0}$ continuity. The slopes of these quantities represent forces (for $u$ and $\gamma$ ) and moments (for $w_{b}^{y}$ and $w_{s}^{y}$ ) and forcing slope continuity will not allow for the flexibility of a discontinuity in the corresponding force or a moment. Thus the element has eight degrees of freedom at each end node and six degrees of freedom at each internal node.

A natural or intrinsic coordinate, $\xi$, is defined, with its origin at the center of the element. This non-dimensional coordinate, $\xi$, ranges from -1 to +1 . The transformation between the two coordinates is given by,

$$
\begin{aligned}
& x_{e}=\frac{l_{e}}{2}(\xi+1) \\
& d x_{e}=\frac{l_{e}}{2}(d \xi)
\end{aligned}
$$

As seen from Figure 3.1, the variables $u, \gamma, w_{b}{ }^{y}$, and $w_{s}^{y}$ have five degrees of freedom each and thus are discretized using a fourth degree polynomial. The variables $w_{b}$ and $w_{s}$ have seven degrees of freedom each and thus are represented by a sixth degree polynomial. Taking $u$ and $w_{b}$, for example, their distributions over the element are represented by,

$$
\begin{aligned}
& u(\xi)=\sum_{i=0}^{4} a_{i} \xi^{i} \\
& w_{b}(\xi)=\sum_{j=0}^{6} b_{j} \xi^{j}
\end{aligned}
$$


These equations can be written in matrix notations as,

$$
\begin{array}{ll}
u(\xi)=\left\lfloor\xi^{i}\right\rfloor\left\{a_{i}\right\} & i=0-4 \\
w_{b}(\xi)=\left\lfloor\xi^{j}\right\rfloor\left\{b_{j}\right\} & j=0-6
\end{array}
$$

where $a_{i}$ and $b_{j}$ are generalized coordinates, which can be derived from the following conditions.

$$
\begin{aligned}
& u(-1)=u_{1} \\
& u(-1 / 2)=u_{2} \\
& u(0)=u_{3} \\
& u(1 / 2)=u_{4} \\
& u(1)=u_{5}
\end{aligned}
$$

Solving the five equations and substituting in the first of Eq. (3.3) we get,

$$
u(\xi)=\left\lfloor H_{L 1}(\xi) \ldots H_{L 5}(\xi)\right\rfloor\left\{\begin{array}{l}
u_{1} \\
\cdot \\
\cdot \\
\cdot \\
u_{5}
\end{array}\right\}
$$

where $H_{L 1}(\xi), H_{L 2}(\xi)$, etc are the shape functions. These shape functions are called as Lagrange shape functions since they satisfy only displacement continuity at the junction between 2 elements. The five Lagrangian polynomials are,

$$
\begin{aligned}
& H_{L 1}=\frac{1}{6} \xi-\frac{1}{6} \xi^{2}-\frac{2}{3} \xi^{3}+\frac{2}{3} \xi^{4} \\
& H_{L 2}=-\frac{4}{3} \xi+\frac{8}{3} \xi^{2}+\frac{4}{3} \xi^{3}-\frac{8}{3} \xi^{4} \\
& H_{L 3}=1-5 \xi^{2}+4 \xi^{4}
\end{aligned}
$$




$$
\begin{aligned}
& H_{L 4}=\frac{4}{3} \xi+\frac{8}{3} \xi^{2}-\frac{4}{3} \xi^{3}-\frac{8}{3} \xi^{4} \\
& H_{L 5}=-\frac{1}{6} \xi-\frac{1}{6} \xi^{2}+\frac{2}{3} \xi^{3}+\frac{2}{3} \xi^{4}
\end{aligned}
$$

The generalized coordinate $b_{j}$ are based on the following conditions

$$
\begin{aligned}
& w_{b}(-1)=w_{b_{1}} \\
& \frac{l_{e}}{2} \frac{d w_{b}}{d \xi}(-1)=w_{b_{1}}^{\prime} \\
& w_{b}(-1 / 2)=w_{b_{2}} \\
& w_{b}(0)=w_{b_{3}} \\
& w_{b}(1 / 2)=w_{b_{4}} \\
& w_{b}(1)=w_{b_{5}} \\
& \frac{l_{e}}{2} \frac{d w_{b}}{d \xi}(1)=w_{b_{5}}^{\prime}
\end{aligned}
$$

Solving these seven equations for $b_{j}$ and substituting into the second of Eq. (3.3) we get,

$$
w_{b}(\xi)=\left\lfloor H_{1}(\xi) \ldots H_{7}(\xi)\right\rfloor\left\{\begin{array}{l}
w_{b_{1}} \\
w_{b_{1}}{ }^{\prime} \\
\cdot \\
\cdot \\
\cdot \\
w_{b_{5}}{ }^{\prime}
\end{array}\right\}
$$

where $H_{1}(\xi), H_{2}(\xi)$, etc., are the shape functions. These shape functions are called as Hermite shape functions since they satisfy inter-element displacement and slope continuities. 
The seven Hermite polynomials used here are,

$$
\begin{aligned}
& H_{1}=\frac{1}{9}\left(\frac{17}{4} \xi-5 \xi^{2}-\frac{79}{4} \xi^{3}+\frac{47}{2} \xi^{4}+11 \xi^{5}-14 \xi^{6}\right) \\
& H_{2}=\frac{l_{e}}{6}\left(\frac{1}{4} \xi-\frac{1}{4} \xi^{2}-\frac{5}{4} \xi^{3}+\frac{5}{4} \xi^{4}+\xi^{5}-\xi^{6}\right) \\
& H_{3}=\frac{16}{9}\left(-\xi+2 \xi^{2}+2 \xi^{3}-4 \xi^{4}-\xi^{5}+2 \xi^{6}\right) \\
& H_{4}=1-6 \xi^{2}+9 \xi^{4}-4 \xi^{6} \\
& H_{5}=\frac{16}{9}\left(\xi+2 \xi^{2}-2 \xi^{3}-4 \xi^{4}+\xi^{5}+2 \xi^{6}\right) \\
& H_{6}=\frac{1}{9}\left(-\frac{17}{4} \xi-5 \xi^{2}+\frac{79}{4} \xi^{3}+\frac{47}{2} \xi^{4}-11 \xi^{5}-14 \xi^{6}\right) \\
& H_{7}=\frac{l_{e}}{6}\left(\frac{1}{4} \xi+\frac{1}{4} \xi^{2}-\frac{5}{4} \xi^{3}-\frac{5}{4} \xi^{4}+\xi^{5}+\xi^{6}\right)
\end{aligned}
$$

\subsection{Element Stiffness Matrix Fornulation}

Stiffness matrix of an element is derived from the virtual strain energy expression of the beam presented in Section 2.5.5. In matrix notation the relation between virtual strain energy of an element and its stiffness matrix can be represented as,

$$
\delta U_{e}=\left\lfloor\delta q_{e}\right\rfloor\left[K_{e}\right]\left\{q_{e}\right\}
$$

where $U_{e}$ represents the element strain energy, $\left\{q_{e}\right\}$ represents the vector of element degrees of freedom and $\left[K_{e}\right]$ represents the element stiffness matrix. The real and virtual displacement fields can be represented, in terms of the shape functions and the corresponding nodal degrees of freedom. 
For example, these expressions for the axial and bending displacements are

$$
\begin{aligned}
& u(x)=\left\lfloor H_{L}\right\rfloor\left\{q_{u}\right\} \\
& \delta u(x)=\left\lfloor\delta q_{u}\right\rfloor\left\{H_{L}\right\} \\
& w_{b}(x)=\lfloor H\rfloor\left\{q_{w_{b}}\right\} \\
& \delta w_{b}(x)=\left\lfloor\delta q_{w_{b}}\right\rfloor\{H\}
\end{aligned}
$$

where $\left\{q_{u}\right\}$ and $\left\{q_{w_{b}}\right\}$ are the vectors of element nodal degrees of freedom for the variables $u$ and $w_{b}$ respectively. Using Eq. (3.11) and similar ones for other variables in the virtual strain energy expression, Eq. (2.35), and then comparing with Eq. (3.10) we can get the stiffness matrix.

\subsubsection{Stiffness Matrix for FSDT}

The element has 29 degrees of freedom. The number of independent variables is five and they are $u, \gamma, w_{b}, w_{s}, w_{b}^{y}$. The $C^{0}$ continuity is used for $u, \gamma$ and $w_{b}^{y}$, and $C^{1}$ continuity is used for $w_{b}$ and $w_{s}$. The element stiffness matrix is divided into twenty-five parts. The stiffness matrix is symmetric about the main diagonal. The following equation shows the element stiffness matrix with its partitioned sub matrices:

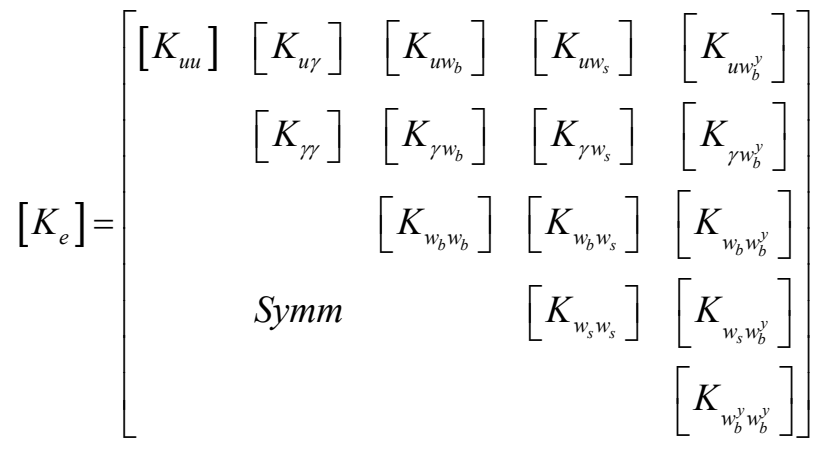


The expression for the various parts of the element stiffness matrix can be derived from the virtual strain energy expression.

The following expressions are obtained,

$$
\begin{aligned}
& {\left[K_{u u}\right]=b \int_{0}^{l_{e}} S_{11}\left\{H_{L}^{\prime}\right\}\left\lfloor H_{L}^{\prime}\right\rfloor d x_{e}} \\
& {\left[K_{u \gamma}\right]=b \int_{0}^{l_{e}} S_{12}\left\{H_{L}^{\prime}\right\}\left\lfloor H_{L}\right\rfloor d x_{e}} \\
& {\left[K_{u w_{b}}\right]=-b \int_{0}^{l_{e}} S_{13}\left\{H_{L}^{\prime}\right\}\left\lfloor H^{\prime \prime}\right\rfloor d x_{e}} \\
& {\left[K_{u w_{s}}\right]=[0]} \\
& {\left[K_{u w_{b}^{\prime \prime}}\right]=-2 b \int_{0}^{l_{e}} S_{14}\left\{H_{L}^{\prime}\right\}\left\lfloor H_{L}^{\prime}\right\rfloor d x_{e}} \\
& {\left[K_{\gamma}\right]=b \int_{0}^{l_{e}} S_{22}\left\{H_{L}\right\}\left\lfloor H_{L}\right\rfloor d x_{e}} \\
& {\left[K_{\gamma w_{b}}\right]=-b \int_{0}^{l_{e}} S_{23}\left\{H_{L}\right\}\left\lfloor H^{\prime \prime}\right\rfloor d x_{e}} \\
& {\left[K_{\gamma w_{s}}\right]=[0]} \\
& {\left[K_{\gamma w_{b}^{\prime \prime}}\right]=-2 b \int_{0}^{l_{e}} S_{24}\left\{H_{L}\right\}\left\lfloor H_{L}^{\prime}\right\rfloor d x_{e}} \\
& {\left[K_{w_{b} w_{b}}\right]=b \int_{0}^{l_{e}} S_{33}\left\{H^{\prime \prime}\right\}\left\lfloor H^{\prime \prime}\right\rfloor d x_{e}+b \int_{0}^{l_{e}} N_{x}\left\{H^{\prime}\right\}\left\lfloor H^{\prime}\right\rfloor d x_{e}} \\
& {\left[K_{w_{b} w_{s}}\right]=[0]} \\
& \left.w_{w_{b}^{\prime}}\right]=2 b \int_{0}^{l_{e}} S_{34}\left\{H^{\prime \prime}\right\}\left\lfloor H_{L}^{\prime}\right\rfloor d x_{e}
\end{aligned}
$$




$$
\begin{aligned}
& {\left[K_{w_{s} w_{s}}\right]=b \int_{0}^{l_{e}} N_{x}\left\{H^{\prime}\right\}\left\lfloor H^{\prime}\right\rfloor d x_{e}+b \int_{0}^{l_{e}} A^{*}\left\{H^{\prime}\right\}\left\lfloor H^{\prime}\right\rfloor d x_{e}} \\
& {\left[K_{w_{s} w_{b}^{\prime}}\right]=[0]} \\
& {\left[K_{w_{b}^{y} w_{b}^{\prime}}\right]=4 b \int_{0}^{l_{e}} S_{44}\left\{H_{L}^{\prime}\right\}\left\lfloor H_{L}^{\prime}\right\rfloor d x_{e}}
\end{aligned}
$$

In the above expressions, the $[S]$ matrix is the reduced material stiffness of the beam and see Chandrasekaran (2000) for details of this and the expression of $A^{*}$.

\subsubsection{Stiffness matrix for HSDT}

The element used for HSDT is shown in Figure 3.1. The element has a total of 34 degrees of freedom. The number of dependent variables is six and they are $u, \gamma, w_{b}, w_{s}$, $w_{b}^{y}$ and $w_{s}^{y}$. The $C^{0}$ continuity is used for $u, \gamma, w_{b}^{y}$. and $w_{s}^{y}$ whereas $C^{1}$ continuity is used for $w_{b}$ and $w_{s}$. The stiffness matrix is partitioned into thirty-six submatrices. The stiffness matrix is symmetric about the main diagonal. The following equation shows the element stiffness matrix:

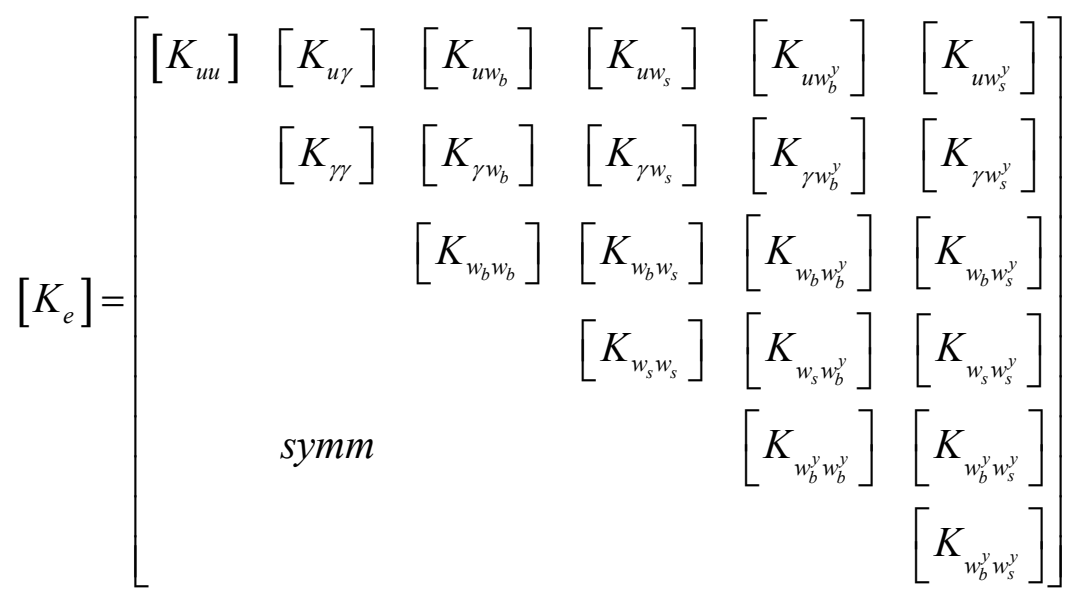


The expressions for the submatrices derived from the virtual strain energy expression are,

$$
\begin{aligned}
& {\left[K_{u u}\right]=b \int_{0}^{l_{e}} T_{11}\left\{H_{L}^{\prime}\right\}\left\lfloor H_{L}^{\prime}\right\rfloor d x_{e}} \\
& {\left[K_{u y}\right]=b \int_{0}^{l_{e}} T_{12}\left\{H_{L}^{\prime}\right\}\left\lfloor H_{L}\right\rfloor d x_{e}} \\
& {\left[K_{u w_{b}}\right]=-b \int_{0}^{l_{e}} T_{13}\left\{H_{L}^{\prime}\right\}\left\lfloor H^{\prime \prime}\right\rfloor d x_{e}} \\
& {\left[K_{u w_{s}}\right]=-b c_{1} \int_{0}^{l_{e}} T_{15}\left\{H_{L}^{\prime}\right\}\left\lfloor H^{\prime \prime}\right\rfloor d x_{e}} \\
& {\left[K_{u w b}\right]=-2 b \int_{0}^{l_{e}} T_{14}\left\{H_{L}^{\prime}\right\}\left\lfloor H_{L}^{\prime}\right\rfloor d x_{e}} \\
& {\left[K_{u w_{s}^{y}}\right]=-2 b c_{1} \int_{0}^{l_{e}} T_{16}\left\{H_{L}^{\prime}\right\}\left\lfloor H_{L}^{\prime}\right\rfloor d x_{e}} \\
& {\left[K_{r y}\right]=b \int_{0}^{l_{e}} T_{22}\left\{H_{L}\right\}\left\lfloor H_{L}\right\rfloor d x_{e}} \\
& {\left[K_{\gamma w_{b}}\right]=-b \int_{0}^{l_{e}} T_{23}\left\{H_{L}\right\}\left\lfloor H^{\prime \prime}\right\rfloor d x_{e}} \\
& {\left[K_{\gamma w_{s}}\right]=-b c_{1} \int_{0}^{l_{e}} T_{25}\left\{H_{L}\right\}\left\lfloor H^{\prime \prime}\right\rfloor d x_{e}} \\
& {\left[K_{\gamma w_{b}^{\prime}}\right]=-2 b \int_{0}^{l_{e}} T_{24}\left\{H_{L}\right\}\left\lfloor H_{L}^{\prime}\right\rfloor d x_{e}} \\
& {\left[K_{\gamma w_{s}^{\prime}}\right]=-2 b c_{1} \int_{0}^{l_{e}} T_{26}\left\{H_{L}\right\}\left\lfloor H_{L}^{\prime}\right\rfloor d x_{e}} \\
& {\left[K_{w_{b} w_{b}}\right]=b \int_{0}^{l_{e}} T_{33}\left\{H^{\prime \prime}\right\}\left\lfloor H^{\prime \prime}\right\rfloor d x_{e}+b \int_{0}^{l_{e}} N_{x}\left\{H^{\prime}\right\}\left\lfloor H^{\prime}\right\rfloor d x_{e}} \\
& {\left[K_{w_{b} w_{s}}\right]=b c_{1} \int_{0}^{l_{e}} T_{35}\left\{H^{\prime \prime}\right\}\left\lfloor H^{\prime \prime}\right\rfloor d x_{e}} \\
& {\left[K_{w_{b} w_{b}^{\prime}}\right]=2 b \int_{0}^{l_{e}} T_{34}\left\{H^{\prime \prime}\right\}\left\lfloor H_{L}^{\prime}\right\rfloor d x_{e}}
\end{aligned}
$$




$$
\begin{aligned}
& {\left[K_{w_{b} w_{s}^{y}}\right]=2 b c_{1} \int_{0}^{l_{e}} T_{36}\left\{H^{\prime \prime}\right\}\left\lfloor H_{L}^{\prime}\right\rfloor d x_{e}} \\
& {\left[K_{w_{s} w_{s}}\right]=b \int_{0}^{l_{e}}\left(N_{X}+D_{55}^{* *}\right)\left\{H^{\prime}\right\}\left\lfloor H^{\prime}\right\rfloor d x_{e}+c_{l}^{2} b \int_{0}^{l} T_{55}\left\{H^{\prime \prime}\right\}\left\lfloor H^{\prime \prime}\right\rfloor d x_{e}} \\
& {\left[K_{w_{s} w_{b}^{y}}\right]=2 b c_{1} \int_{0}^{l_{e}} T_{54}\left\{H^{\prime \prime}\right\}\left\lfloor H_{L}^{\prime}\right\rfloor d x_{e}} \\
& {\left[K_{w_{s} w_{s}^{y}}\right]=2 b c_{1}^{l_{e}} \int_{0}^{l_{5}} T_{56}\left\{H^{\prime \prime}\right\}\left\lfloor H_{L}^{\prime}\right\rfloor d x_{e}} \\
& {\left[K_{w_{b}^{y} w_{b}^{y}}\right]=4 b \int_{0}^{l_{e}} T_{44}\left\{H_{L}^{\prime}\right\}\left\lfloor H_{L}^{\prime}\right\rfloor d x_{e}} \\
& {\left[K_{w_{b}^{y} w_{s}^{y}}\right]=4 b c_{1} \int_{0}^{l_{e}} T_{46}\left\{H_{L}^{\prime}\right\}\left\lfloor H_{L}^{\prime}\right\rfloor d x_{e}} \\
& {\left[K_{w_{s}^{y} w_{s}^{y}}\right]=c_{1}^{2} 4 b \int_{0}^{l_{e}} T_{66}\left\{H_{L}^{\prime}\right\}\left\lfloor H_{L}^{\prime}\right\rfloor d x_{e}}
\end{aligned}
$$

where $c_{1}=\frac{4}{3 h^{2}}$.

\subsection{Element Inertia Matrix Formulation}

The expression for the variation in total kinetic energy, $\delta T$, presented in Section 2.5.6 is used to derive the element inertia matrix. In matrix notation the relation between variational kinetic energy of an element and its inertia matrix $\left[M_{e}\right]$ can be represented as,

$$
-\delta T_{e}=\left\lfloor\delta q_{e}\right\rfloor\left[M_{e}\right]\left\{\ddot{q}_{e}\right\}
$$

Where $T_{e}$ represents the element kinetic energy, $\left\{q_{e}\right\}$ represents the vector of element degrees of freedom, and $\left[M_{e}\right]$ the element inertia matrix. Using Eq. (3.11) and similar expression for other variables in Eq. (3.16), we can obtain the element inertia matrix. 


\subsubsection{Inertia Matrix for FSDT}

The element inertia matrix for FSDT is derived following a procedure similar to the one for the element stiffness matrix. The inertia matrix is divided into twenty-five parts similar to the stiffness matrix. The inertia matrix is also symmetric about the main diagonal. The following equation shows the inertia matrix and its elements:

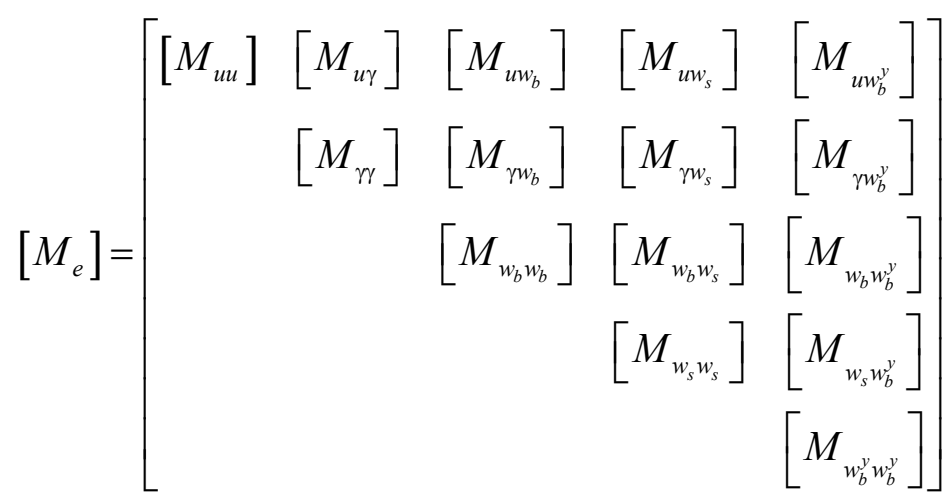

The expressions for the different submatrices derived from the variational kinetic energy term are:

$$
\begin{aligned}
& {\left[M_{u u}\right]=b \int_{0}^{l_{e}} I_{0}\left\{H_{L}\right\}\left\lfloor H_{L}\right\rfloor d x_{e}} \\
& {\left[M_{u w_{b}}\right]=-b \int_{0}^{l_{e}} I_{1}\left\{H_{L}\right\}\left\lfloor H^{\prime}\right\rfloor d x_{e}} \\
& {\left[M_{w_{b} w_{b}}\right]=b \int_{0}^{l_{e}} I_{2}\left\{H^{\prime}\right\}\left\lfloor H^{\prime}\right\rfloor d x_{e}+b \int_{0}^{l_{e}} I_{0}\{H\}\lfloor H\rfloor d x_{e}} \\
& {\left[M_{w_{b} w_{s}}\right]=b \int_{0}^{l_{e}} I_{0}\{H\}\lfloor H\rfloor d x_{e}} \\
& {\left[M_{w_{s} w_{s}}\right]=\int_{0}^{l_{e}} I_{0}\{H\}\lfloor H\rfloor d x_{e}}
\end{aligned}
$$




$$
\begin{aligned}
& {\left[M_{w_{b}^{y} w_{b}^{\prime y}}\right]=b \int_{0}^{l_{e}} I_{2}\left\{H_{L}\right\}\left\lfloor H_{L}\right\rfloor d x_{e}} \\
& {\left[M_{u \gamma}\right]=\left[M_{u w_{s}}\right]=\left[M_{u w_{b}^{\prime}}\right]=\left[M_{\gamma}\right]=\left[M_{\gamma w_{b}}\right]=[0]} \\
& {\left[M_{\gamma w_{s}}\right]=\left[M_{\gamma w_{b}^{y}}\right]=\left[M_{w_{b} w_{b}^{v}}\right]=\left[M_{w_{s} w_{b}^{y}}\right]=[0]}
\end{aligned}
$$

\subsubsection{Inertia Matrix for HSDT}

In case of HSDT, $C^{0}$ continuity is used for $u, \gamma, w_{s}{ }^{y}, w_{b}^{y}$ degrees of freedom and

$C^{1}$ continuity is used for $w_{b}$ and $w_{s}$ degrees of freedom. The element inertia matrix for HSDT is derived following a procedure similar to the one for the element stiffness matrix. The inertia matrix is divided into thirty-six parts. The inertia matrix is symmetric about the main diagonal. The following equation shows the inertia matrix and its elements:

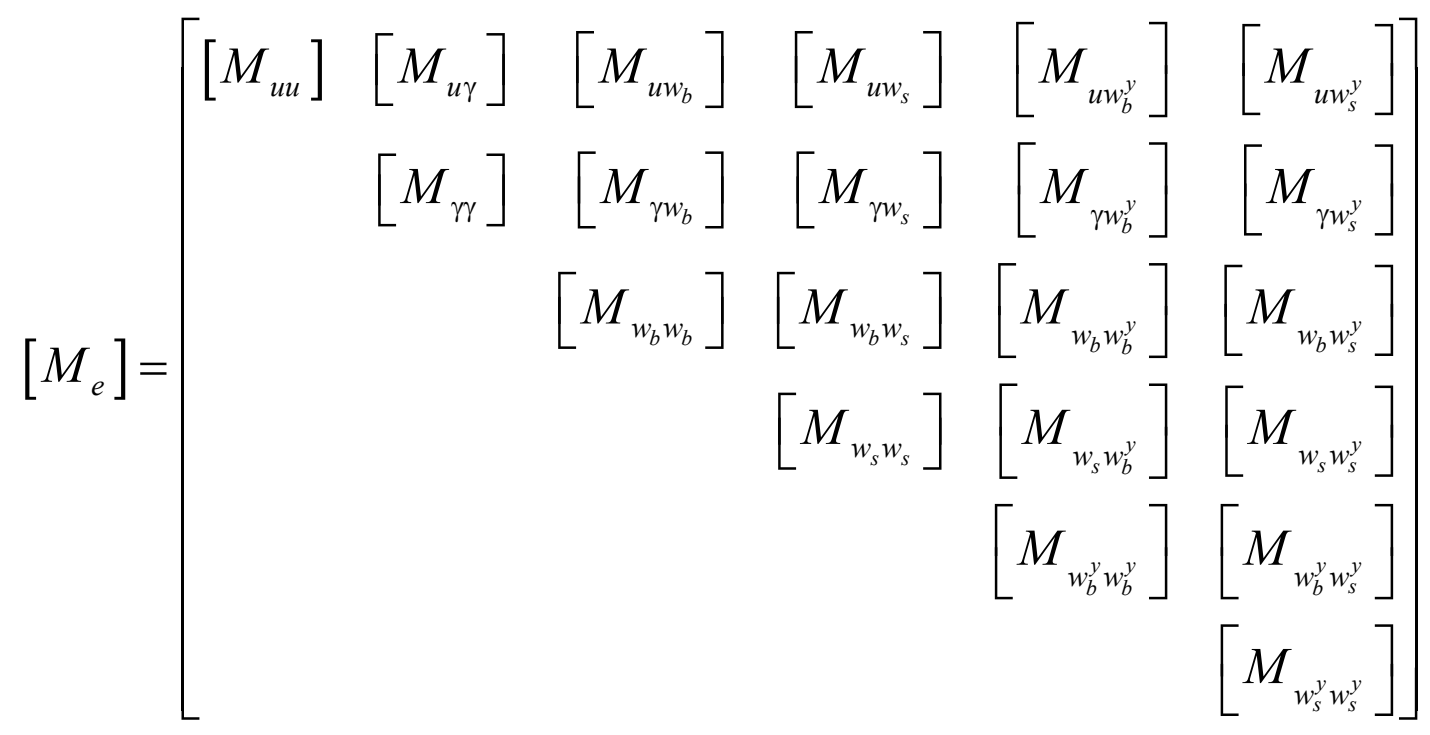


The expression for the submatrices derived from the virtual kinetic energy expression are:

$$
\begin{aligned}
& {\left[M_{u u}\right]=b \int_{0}^{l_{e}} I_{0}\left\{H_{L}\right\}\left\lfloor H_{L}\right\rfloor d x_{e}} \\
& {\left[M_{u w_{b}}\right]=-b \int_{0}^{l_{e}} I_{1}\left\{H_{L}\right\}\left\lfloor H^{\prime}\right\rfloor d x_{e}} \\
& {\left[M_{u w_{s}}\right]=-b c_{1} \int_{0}^{l_{e}} I_{3}\left\{H_{L}\right\}\left\lfloor H^{\prime}\right\rfloor d x_{e}} \\
& {\left[M_{w_{b} w_{b}}\right]=b \int_{0}^{l_{e}} I_{2}\left\{H^{\prime}\right\}\left\lfloor H^{\prime}\right\rfloor d x_{e}+b \int_{0}^{l_{e}} I_{0}\{H\}\lfloor H\rfloor d x_{e}} \\
& {\left[M_{w_{b} w_{s}}\right]=b \int_{0}^{l_{e}} c_{1} I_{4}\left\{H^{\prime}\right\}\left\lfloor H^{\prime}\right\rfloor d x_{e}+b \int_{0}^{l_{e}} I_{0}\{H\}\lfloor H\rfloor d x_{e}} \\
& {\left[M_{w_{s} w_{s}}\right]=b \int_{0}^{l_{e}} c_{1}^{2} I_{6}\left\{H^{\prime}\right\}\left\lfloor H^{\prime}\right\rfloor d x_{e}+b \int_{0}^{l_{e}} I_{0}\{H\}\lfloor H\rfloor d x_{e}} \\
& {\left[M_{w_{b}^{y} w_{b}^{y}}\right]=b \int_{0}^{l_{e}} I_{2}\left\{H_{L}\right\}\left\lfloor H_{L}\right\rfloor d x_{e}} \\
& {\left[M_{w_{b}^{y} w_{s}^{v}}\right]=b c_{1} \int_{0}^{l_{e}} I_{4}\left\{H_{L}\right\}\left\lfloor H_{L}\right\rfloor d x_{e}} \\
& {\left[M_{w_{s}^{y} w_{s}^{y}}\right]=b c_{1}^{2} \int_{0}^{l_{e}} I_{6}\left\{H_{L}\right\}\left\lfloor H_{L}\right\rfloor d x_{e}} \\
& {\left[M_{u \gamma}\right]=\left[M_{u w_{s}}\right]=\left[M_{u w_{b}^{\prime}}\right]=\left[M_{u w_{s}^{\prime}}\right]=\left[M_{\gamma}\right]=\left[M_{\gamma w_{b}}\right]=[0]} \\
& {\left[M_{\gamma w_{s}}\right]=\left[M_{\gamma w_{b}^{y}}\right]=\left[M_{\gamma w_{s}^{v}}\right]=\left[M_{w_{b} w_{b}^{y}}\right]=\left[M_{w_{b} w_{s}^{y}}\right]=\left[M_{w_{s} w_{b}^{y}}\right]=[0]} \\
& \text { where } c_{1}=\frac{4}{3 h^{2}} \text {. }
\end{aligned}
$$




\subsection{Global Equations of Motion}

The element stiffness matrix $\left[K_{e}\right]$ and the element inertia matrix $\left[M_{e}\right]$ derived in the previous sections are for individual elements. The element stiffness matrices for all the elements are assembled to form the global stiffness matrix $[K]$. Similarly the element inertia matrices for all the elements are assembled to form the global inertial matrix $[M]$. Then the finite element equations of motion are given by:

$$
[M]\{\ddot{q}\}+[C]\{\dot{q}\}+[K]\{q\}=\{Q\}
$$

where $[\mathrm{C}]$ is the damping matrix, $\{q\}$ is the global displacement vector and $\{\mathrm{Q}\}$ the

global load vector. In the present analysis damping is not considered and there is no external load, and therefore the load vector is a zero vector. Thus the equations of motion for the moving beam reduce to:

$$
[M]\{\ddot{q}\}+[K]\{q\}=\{0\}
$$

\subsection{Lagrange Multipliers}

Analysis of engineering problems requires imposing specific constraints on the solution variables. Improper specification of constraints or boundary conditions will lead to erroneous results. The boundary conditions can be applied to system using the elimination approach, penalty approach and Lagrange approach.

In the elimination approach, the zero constraints are enforced by deleting or eliminating the rows and columns of the corresponding degrees of freedom. The stiffness matrix obtained after removal of rows and columns will be non-singular, provided the boundary conditions are applied properly. In the case of a static problem, the displacement vector can be solved for using Gaussian elimination from the reduced 
stiffness matrix. The support reactions can be calculated using the original stiffness matrix. This method is suitable when the supports fall exactly on the nodes.

In the penalty approach, the boundary conditions are applied by adding a constant of relatively large magnitude to the diagonal elements of the stiffness matrix and a corresponding force is added to obtain the required displacement constraint. Then the reactions at the support locations can be calculated. This method is simple and it does not need any additional equations and the bandwidth of the stiffness matrix remains the same. The penalty approach is an approximate approach. The accuracy of the solution depends on the choice of the constants used.

In the Lagrange multiplier method, additional variables known as Lagrange multipliers are added to incorporate the boundary conditions. Lagrange multiplier approach is used in the present work. In the present research, the support locations change with time, and therefore they do not always fall exactly at the nodes. For this type of system the Lagrange multiplier approach is more amenable. The Hamiltonian of the beam, [Eq. (2.6)] is

$$
\Delta_{p}=(\delta U-\delta T-\delta W)
$$

Where $\delta U, \delta T$, and $\delta W$ represent the variation in strain energy, variation in kinetic energy and virtual external work respectively. To apply the boundary conditions via Lagrange multipliers, additional variables are included in the Hamiltonian and the modified Hamiltonian is given by,

$$
\bar{\Delta}_{p}=(\delta U-\delta T-\delta W)+\delta(\ldots)
$$

Where the terms in the second set of parenthesis depend on the plate theories used. 
In the case of FSDT the constraints are applied to $\mathrm{w}_{\mathrm{b}}, \mathrm{w}_{\mathrm{s}}$ and $\mathrm{w}_{\mathrm{b}}{ }^{\mathrm{y}}$ and the modified Hamiltonian is given by,

$$
\bar{\Delta}_{p}=(\delta U-\delta T-\delta W)+\delta\left(\left.\lambda_{1} w_{b}\right|_{x_{s_{1}}}+\left.\lambda_{2} w_{s}\right|_{x_{s_{1}}}+\left.\lambda_{3} w_{b}^{y}\right|_{s_{s_{1}}}+\left.\lambda_{4} w_{b}\right|_{x_{s_{2}}}+\left.\lambda_{5} w_{s}\right|_{x_{s_{2}}}+\left.\lambda_{6} w_{b}^{y}\right|_{x_{s_{2}}}\right)
$$

Where $\lambda_{i}$ 's represent the additional variables or Lagrange degrees of freedom at the two support locations $x_{s_{1}}$ and $x_{s_{2}}$. In the case of HSDT, the constraints are applied to $\mathrm{w}_{\mathrm{b}}, \mathrm{w}_{\mathrm{S}}$, $\mathrm{w}_{\mathrm{b}}{ }^{\mathrm{y}}$ and $\mathrm{w}_{\mathrm{s}}{ }^{\mathrm{y}}$ and the modified Hamiltonian is given by,

$$
\begin{aligned}
& \bar{\Delta}_{p}=(\delta U-\delta T-\delta W) \\
& +\delta\left(\left.\lambda_{1} w_{b}\right|_{x_{s 1}}+\left.\lambda_{2} w_{s}\right|_{x_{s 1}}+\left.\lambda_{3} w_{b}^{y}\right|_{x_{s 1}}+\left.\lambda_{4} w_{s}^{y}\right|_{x_{s 1}}+\left.\lambda_{5} w_{b}\right|_{x_{s 2}}+\left.\lambda_{6} w_{s}\right|_{x_{s 2}}+\left.\lambda_{7} w_{b}^{y}\right|_{x_{s 2}}+\left.\lambda_{8} w_{s}^{y}\right|_{x_{s 2}}\right)
\end{aligned}
$$

These new variables at the supports alter the global stiffness and mass matrix. In the case of a non-moving beam, the Lagrange multipliers are applied to both inertia and stiffness matrices. This is to apply the constraints on displacements, velocities and acceleration at the supports. The modified global stiffness and inertia matrices are given by,

$$
\begin{aligned}
& {\left[K_{m}\right]=\left[\begin{array}{cc}
{[K]} & {\left[K_{\lambda}\right]} \\
{\left[K_{\lambda}^{T}\right]} & {[0]}
\end{array}\right]} \\
& {\left[M_{m}\right]=\left[\begin{array}{cc}
{[M]} & {\left[M_{\lambda}\right]} \\
{\left[M_{\lambda}^{T}\right]} & {[0]}
\end{array}\right]}
\end{aligned}
$$

Where, $\left[\mathrm{K}_{\lambda}\right],\left[\mathrm{M}_{\lambda}\right]$ are the stiffness and inertia matrices, respectively, corresponding to the Lagrange multipliers.

In case of moving beam, the constraints are applied only to displacements, i.e., the Lagrange multipliers are applied only to stiffness matrices. The velocities and 
acceleration are not constrained. The modified global stiffness and inertia matrices are represented by,

$$
\begin{aligned}
& {\left[K_{m}\right]=\left[\begin{array}{cc}
{[K]} & {\left[K_{\lambda}\right]} \\
{\left[K_{\lambda}^{T}\right]} & {[0]}
\end{array}\right]} \\
& {\left[M_{m}\right]=\left[\begin{array}{ll}
{[M]} & {[0]} \\
{[0]} & {[0]}
\end{array}\right]}
\end{aligned}
$$

With the addition of the lagrange multiplier degrees of freedoms Eq. (3.22) modifies to

$$
\left[M_{m}\right]\left\{\ddot{q}_{m}\right\}+\left[K_{m}\right]\left\{q_{m}\right\}=\{0\}
$$

where $\left\lfloor q_{m}\right\rfloor=\left\lfloor\lfloor q\rfloor \lambda_{1} \lambda_{2} \ldots \lambda_{n}\right\rfloor$

The Lagrange multiplier approach works well if the number of constraints are less than number of actual degrees of freedom. For statics cases like a simply supported beam subjected to a uniform distributed load or point load, the Lagrange multiplier values are the reactions at the support locations. Using the Lagrange multiplier method, the additional steps to determine the reactions can be avoided.

\subsection{Gaussian Quadrature}

The computation of element inertial and mass matrices involves spatial integration. The Gaussian quadrature method is used for this integration. Gaussian quadrature method has been found to be more accurate for finite element work compared to other numerical integration procedures. In Gaussian quadrature, the positions of the sampling points and the weights have been optimized. In Gaussian quadrature the integrals can be extended to two and three dimensions easily. 
Gaussian quadrature requires $n$ sampling points to integrate a polynomial of order $(2 n-1)$ exactly. In the present case the highesst order polynomial to be integrated is fourteen. Therefore a seven point Gaussian quadrature scheme is used. The integration scheme is represented as,

$$
\int_{-1}^{1} f(\xi) d \xi=\sum_{j=1}^{n} w_{j} f\left(a_{j}\right)
$$

where $n$ is the number of sampling points, $a_{j}$ represent the $\xi$ coordinate at the sampling points and $w_{j}$ the corresponding weights. The sampling points and weights used are shown below

\begin{tabular}{|l|l|}
\hline Sampling Points & Weights \\
\hline \pm 0.9491079123 & 0.1294849661 \\
\hline \pm 0.7415311855 & 0.0797053914 \\
\hline \pm 0.4058451513 & 0.3813005050 \\
\hline 0.0000000000 & 0.4179591836 \\
\hline
\end{tabular}

\section{Table 3.1 Gauss integration points and weights}

The stiffness and the inertia matrices are integrated after transforming the coordinates from $x_{e}$ to the non-dimensional coordinate $\xi$.

\subsection{Time Integration Schemes}

To solve the dynamic equilibrium equations, two different time integration schemes, namely, the Wilson Theta method and Newmark's method are used. These methods are referred to as direct methods, since they solve the differential equations directly and do not need any transformation to a different form. The methods are based 
on certain concepts, which are discussed by Bathe (2001). These methods are based on, the following assumptions

1. The equilibrium conditions are satisfied at discrete time intervals.

2. The variations of displacements, velocities and accelerations are assumed within the time interval.

The Wilson theta method and Newark's method are both extensions of the linear acceleration method. These methods are implicit integration methods which use the equilibrium conditions at time $(t+\Delta t)$. These methods are unconditionally stable.

In the Wilson-Theta method the acceleration is assumed to vary linearly between time $t$ and $(t+\Delta t)$. To calculate the displacement, velocity and acceleration vectors at time $(t+\Delta t)$, the equilibrium equations at time $(t+\theta \Delta t)$ is considered. A theta value of 1.4 is used as recommended by Bathe (2001) to obtain unconditional stability. The assumption used is represented by,

$$
{ }^{t+\tau} \ddot{q}={ }^{t} \ddot{q}+\frac{\tau}{\theta \Delta t}\left({ }^{t+\theta \Delta t} \ddot{q}-{ }^{t} \ddot{q}\right)
$$

Where $\tau$ denotes an increment in time. When $\theta$ is set to 1 the method reduces to linear acceleration method.

In Newark's method the following assumptions are used,

$$
\begin{aligned}
& { }^{t+\Delta t} \dot{q}={ }^{t} \dot{q}+\left[\left[(1-\delta){ }^{t} \ddot{q}+\delta^{t+\Delta t} \ddot{q}\right] \Delta t\right. \\
& { }^{t+\Delta t} q={ }^{t} q+{ }^{t} \dot{q} \Delta t+\left[\frac{1}{2}\left[(1-\alpha){ }^{t} \ddot{q}+\delta^{t+\Delta t} \ddot{q}\right] \Delta t^{2}\right.
\end{aligned}
$$

The parameters $\alpha$ and $\delta$ are determined based on required accuracy and stability. In this work $\alpha$ is taken as 0.25 and $\delta$ is taken as 0.5 . 


\subsection{Boundary Conditions and Initial Conditions}

The boundary conditions used to solve the finite element equations of motion for all the plate theories are listed below.

\subsubsection{Boundary Conditions for CLPT}

The boundary conditions used for the beam using CLPT are:

Hinged Support $\quad: \quad u=w=0$

Fixed Support $\quad: \quad u=w=w^{\prime}=0$

\subsubsection{Boundary Conditions for FSDT}

The boundary conditions used for the beam using FSDT are:

$\begin{array}{ll}\text { Hinged Support } & : \quad u=w_{b}=w_{s}=w_{b}^{y}=0 \\ \text { Fixed Support } & : \quad u=w_{b}=w_{s}=w_{b}^{\prime}=w_{s}^{\prime}=w_{b}^{y}=0\end{array}$

\subsubsection{Boundary Conditions for HSDT}

The boundary conditions used for the formulation based on HSDT are:

$\begin{array}{ll}\text { Hinged Support } & : \quad u=w_{b}=w_{s}=w_{b}^{y}=w_{s}^{y}=0 \\ \text { Fixed Support } & : \quad u=w_{b}=w_{s}=w_{b}^{\prime}=w_{s}^{\prime}=w_{b}^{y}=w_{s}^{y}=0\end{array}$ 


\subsubsection{Initial Conditions}

For the formulation based on CLPT the first mode shape in bending $(w)$ of the symmetrically placed overhang beam normalized such that the left and right end deflections are $0.01 \mathrm{~m}$ is taken as the initial shape. The first mode shape of $w_{b}$ of the symmetrically placed overhang beam is taken as the initial shape for FSDT and HSDT. The shape is normalized such that the tip deflections are $0.01 \mathrm{~m}$. 


\section{RESULTS AND DISCUSSION}

\subsection{Introduction}

A finite element code is written in MATLAB to generate the necessary numerical results based on the finite-element model formulated in the previous chapter of a composite moving beam. Validation of the current model based on HSDT is intrinsically done by generating results for isotropic, CLPT and FSDT cases and comparing with the existing results in the literature. Tables and graphs are presented for the tip deflections of a moving beam subjected to an initial bent shape in the lateral direction.

\subsection{Isotropic Beam Comparison}

The response analysis of an initially-deformed isotropic overhang beam moving axially relative to the supports is performed. The geometric and material characteristics of the beam are taken from Sreeram and Sivaneri (1997). Referring to Figure 4.1,

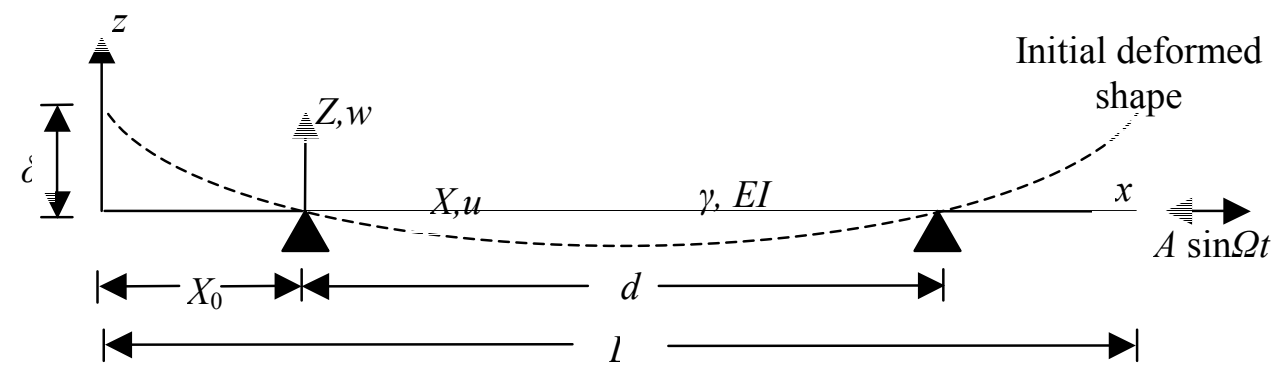

Figure 4.1 Isotropic Moving Beam 
Length of the beam $(L)=1.0 \mathrm{~m}$

Mass per unit length of the beam $(\gamma)=1.0 \mathrm{Kg} / \mathrm{m}$

Beam Stiffness $(E I)=1.0 \mathrm{Nm}^{2}$

Distance between the supports $(d)=0.25 L$

Initial distance of the left end of the beam from the first support $\left(X_{0}\right)=0.375 \mathrm{~L}$

Initial transverse deflection at the left end of the beam $(\delta)=0.01 \mathrm{~m}$

The beam is considered to perform rigid body sinusoidal oscillations in the longitudinal direction about the support with a frequency of $\Omega$ and an amplitude of $A$. The value of the amplitude, $A$, is taken to be $0.05 \mathrm{~m}$. The initial shape is the first transverse mode shape at $t=0$, normalized such that the left tip deflection, $\delta$, is $0.01 \mathrm{~m}$. The transverse deflection at the left end of the beam is plotted against time for various values of $\Omega$ (axial frequency) of the beam. The time response is obtained using the Wilson-theta method with theta a value of 1.4. A time step of $2.5 \times 10^{-4} \mathrm{sec}$ is used to study the behavior. Figures 4.2 to 4.5 show the left tip deflection in the transverse direction as a function of time for axial frequencies of 10, 20, 22 and $30 \mathrm{rad} / \mathrm{sec}$. The results of the Sreeram and Sivaneri (1997) or Sreeram (1995) are also shown in these figures and a comparison indicates excellent agreement.

The above beam parameters were first used by Buffinton and Kane (1985) and then, for comparison purposes, were adopted by Lee, (1992) and Sreeram and Sivaneri (1997). The beam stiffness value of $1 \mathrm{Nm}^{2}$ and mass per unit length of $1 \mathrm{Kg} / \mathrm{m}$ represent generic and artificial values. Consequently these values are much lower than that of a real beam made of steel or aluminum. Figures 4.2 to 4.5 indicate that the oscillations of this generic beam become unstable at values of axial frequencies $(\Omega) 20 \mathrm{rad} / \mathrm{sec}$ or higher. 
The above three references did not verify if a beam with realistic material properties would behave in a similar manner. To answer this question, the response of a steel beam with the following properties is studied:

Young's Modulus $(E)=200 \mathrm{GPa} \quad$ Poisson's ratio $(v)=0.3$

Mass density $(\rho)=7900 \mathrm{Kg} / \mathrm{m}^{3} \quad$ Length of the beam $(L)=1.0 \mathrm{~m}$

Beam height $(h)=0.06 \mathrm{~m} \quad$ Beam width $(b)=0.05 \mathrm{~m}$

Distance between the supports $(d)=0.25 \mathrm{~L}$

Initial distance of first support from left end of the beam $\left(X_{0}\right)=0.375 \mathrm{~L}$

Amplitude of axial motion $(A)=0.05 \mathrm{~m}$

Initial transverse deflection at the left end of the beam $(\delta)=0.01 \mathrm{~m}$

The response of the steel beam is obtained for axial rigid-body motion frequencies of $0,5,10,15,20$, and $25 \mathrm{rad} / \mathrm{sec}$. The transverse deflection of the left end of the beam for the above frequencies are presented in Figures 4.6, 4.8, 4.10, 4.12, 4.14, and 4.16, respectively. As expected the non-moving beam $(\Omega=0)$ response, as seen from Figure 4.6, is sinusoidal. Even a cursory comparison of the response of the generic moving beam (Figures 4.2 to 4.4 ) with that of the steel moving beam shows two prominent qualitative differences. The generic moving beam starts to become unstable at about 1.5 secs at axial frequencies higher than or equal to $20 \mathrm{rad} / \mathrm{sec}$ whereas the steel moving beam is stable well beyond 1.5 secs even for the highest axial frequency $(\Omega=25 \mathrm{rad} / \mathrm{sec})$ considered (Figure 4.16). The steel beam exhibits a beat like phenomenon while the generic beam apparently does not manifest such a response.

As seen in Figure 4.7, the transverse first natural frequency of an axially nonmoving overhang beam is a constant with respect to time with a value of $1415.8 \mathrm{rad} / \mathrm{sec}$. 
On the other hand, the transverse first natural frequency of an axially moving beam varies with time due to two reasons. The first one is changing with time of the relative support locations in reference to the left tip of the beam. The second is the change in the bending stiffness of the beam caused by the inertia force generated due to the axial acceleration of the beam which varies with time. The instantaneous natural frequency is plotted as a function of time in Figures 4.9, 4.11, 4.13, 4.15 and 4.17 for $\Omega$ values of 5, 10, 15, 20, and $25 \mathrm{rad} / \mathrm{sec}$, respectively.

The key aspects of the results from Figures 4.6 to 4.17 are summarized in Table 4.1. The first two columns list the axial frequency $(\Omega)$ in $\mathrm{rad} / \mathrm{sec}$ and cycles/sec $(\mathrm{Hz})$, respectively. The corresponding period $\left(T_{\Omega}\right)$ is listed in the third column. This is the larger of the two periods seen in each of the beat-type response figures of the moving steel beam. The transverse natural frequency of the non-moving $(\Omega=0)$ overhang beam is $1416 \mathrm{rad} / \mathrm{sec}$ and listed as the first entry of the fourth column. When axial rigid body motion is present $(\Omega \neq 0)$, the instantaneous transverse natural frequency varies with time, as mentioned earlier. The rest of the entries of the fourth column depict the range of this natural frequency in $\mathrm{rad} / \mathrm{sec}$. The same natural frequency range in cycles/sec is shown in the fifth column. For the range of $\Omega$ from 0 to $25 \mathrm{rad} / \mathrm{sec}$, the $\omega_{1}$ varies from 197.83 to $225.36 \mathrm{~Hz}$. It is noted that the value of $\Omega$ of the moving beam does not seem to have an effect on the range of the instantaneous transverse natural frequency even though $\Omega$ changes the inertial force leading to a corresponding change in the bending stiffness of the beam. A close examination of the relative beam stiffness terms indicated that the beam stiffness due to $E I$ is six orders higher than that due to inertial force. 


\begin{tabular}{|c|c|c|c|c|c|}
\hline \multicolumn{2}{|c|}{$\Omega$ (forcing - axial) } & $T_{\Omega}$ & \multicolumn{2}{|c|}{$\omega_{1}$ (natural) } & $\omega$ (response) \\
\hline $\mathrm{rad} / \mathrm{sec}$ & $\mathrm{Hz}$ & $\mathrm{sec}$ & $\mathrm{rad} / \mathrm{sec}$ & $\mathrm{Hz}$ & $\mathrm{Hz}$ \\
\hline 0 & 0 & & 1416 & 225.36 & 226 \\
\hline 5 & 0.79 & 1.260 & $1243-1416$ & $197.83-225.36$ & 211 \\
\hline 10 & 1.59 & 0.628 & $1243-1416$ & $197.83-225.36$ & 211 \\
\hline 15 & 2.39 & 0.419 & $1243-1416$ & $197.83-225.36$ & 211 \\
\hline 20 & 3.18 & 0.314 & $1243-1416$ & $197.83-225.36$ & 211 \\
\hline 25 & 3.98 & 0.251 & $1243-1416$ & $197.83-225.36$ & 211 \\
\hline
\end{tabular}

Table 4.1 Summary of steel moving beam response

The approximate values of the response frequency $(\omega)$ is obtained by counting the number of peaks from the response figures of the moving beam and these are entered in the final column of Table 4.1. This $\omega$ is 226 for $\Omega=0$ and changes to a value of 211 when $\Omega$ is non zero. Thus the beat like response of the moving beam is due to the interplay of the two frequencies, namely the axial motion and the transverse natural frequency. The smaller period of the beat is close to that of the first natural frequency $\left(\omega_{1}\right)$ and larger period is that of the axial frequency.

\begin{tabular}{|c|c|c|c|}
\hline $\begin{array}{c}\Omega \\
(\mathrm{rad} / \mathrm{sec})\end{array}$ & $\begin{array}{c}w_{(t=0)} \\
(\mathrm{m})\end{array}$ & $\begin{array}{c}w_{\max } \\
(\mathrm{m})\end{array}$ & $\begin{array}{c}\text { Magnification } \\
\text { Factor } \\
(w)_{\max }\end{array}$ \\
\hline 0 & 0.01 & 0.0100 & 1.00 \\
\hline 5 & 0.01 & 0.0140 & 1.40 \\
\hline 10 & 0.01 & 0.0141 & 1.41 \\
\hline 15 & 0.01 & 0.0141 & 1.41 \\
\hline 20 & 0.01 & 0.0141 & 1.41 \\
\hline 25 & 0.01 & 0.0142 & 1.42 \\
\hline
\end{tabular}

Table 4.2 Maximum tip deflection of steel beam

As seen from the response figures, the left tip of the beam undergoes oscillations with an amplitude that varies with time. Over the time interval of $1 \mathrm{sec}$, the absolute maximum value of the left tip of the beam is captured and reported in the third column of 


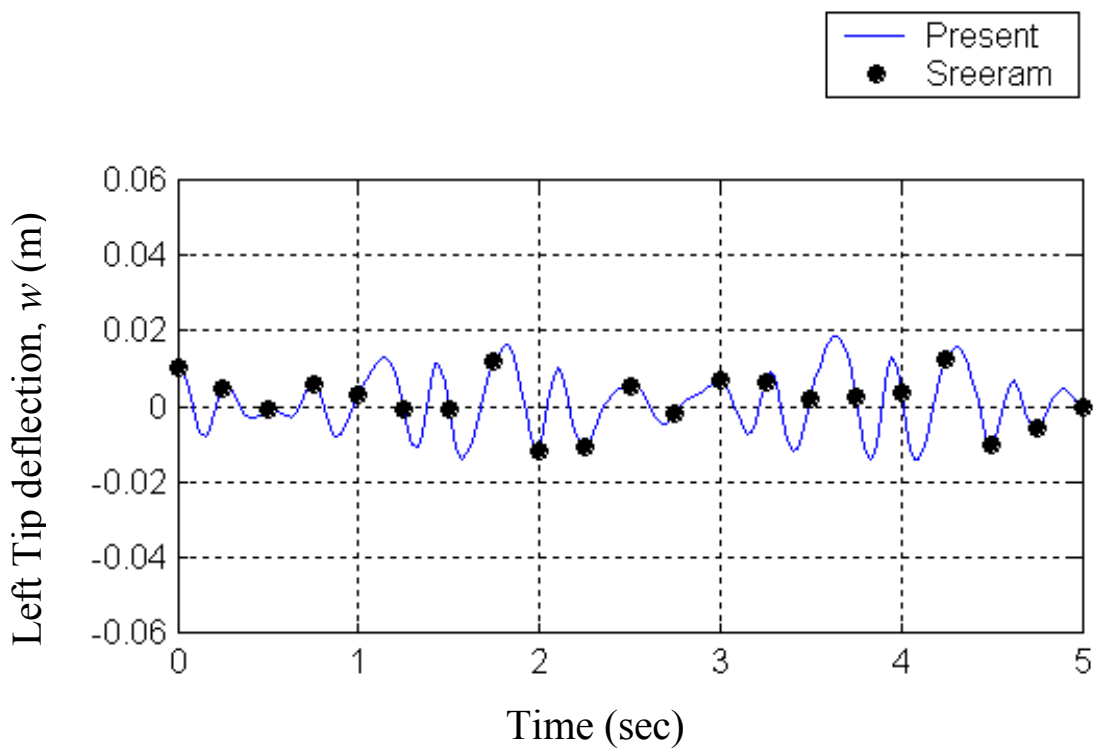

Figure 4.2 Response analysis of initially deformed isotropic moving beam with $\Omega$ $=10 \mathrm{rad} / \mathrm{sec}$
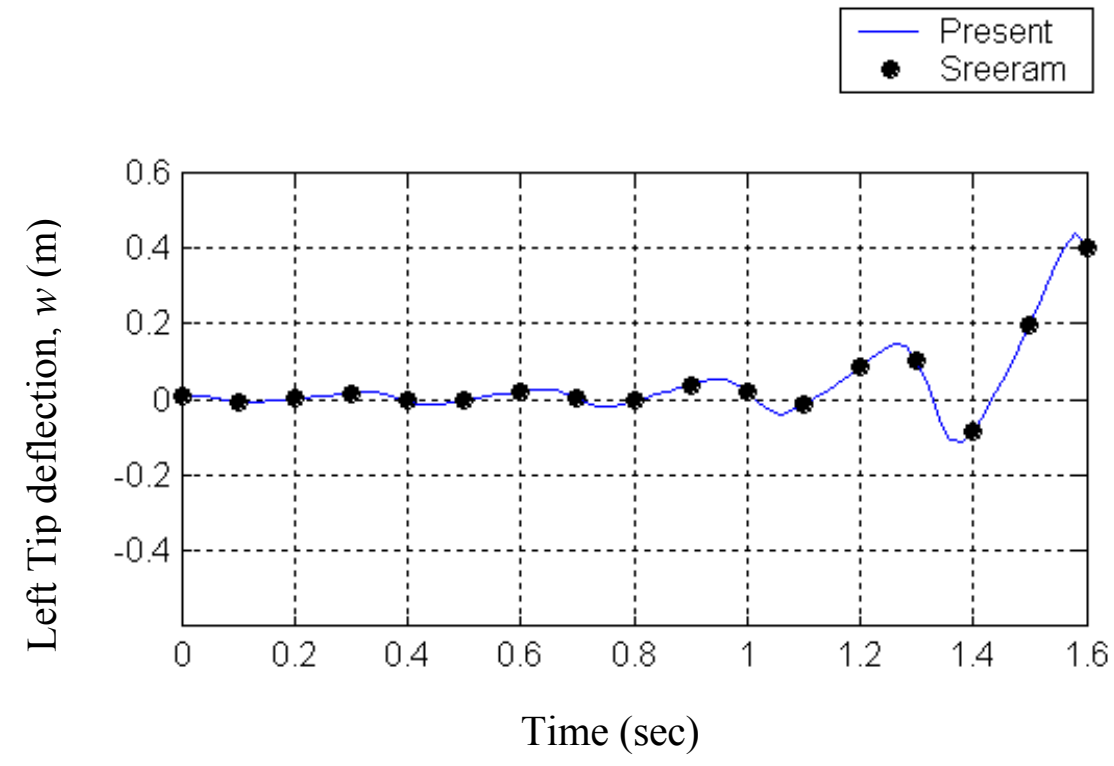

Figure 4.3 Response analysis of initially deformed isotropic moving beam with $\Omega=20 \mathrm{rad} / \mathrm{sec}$ 


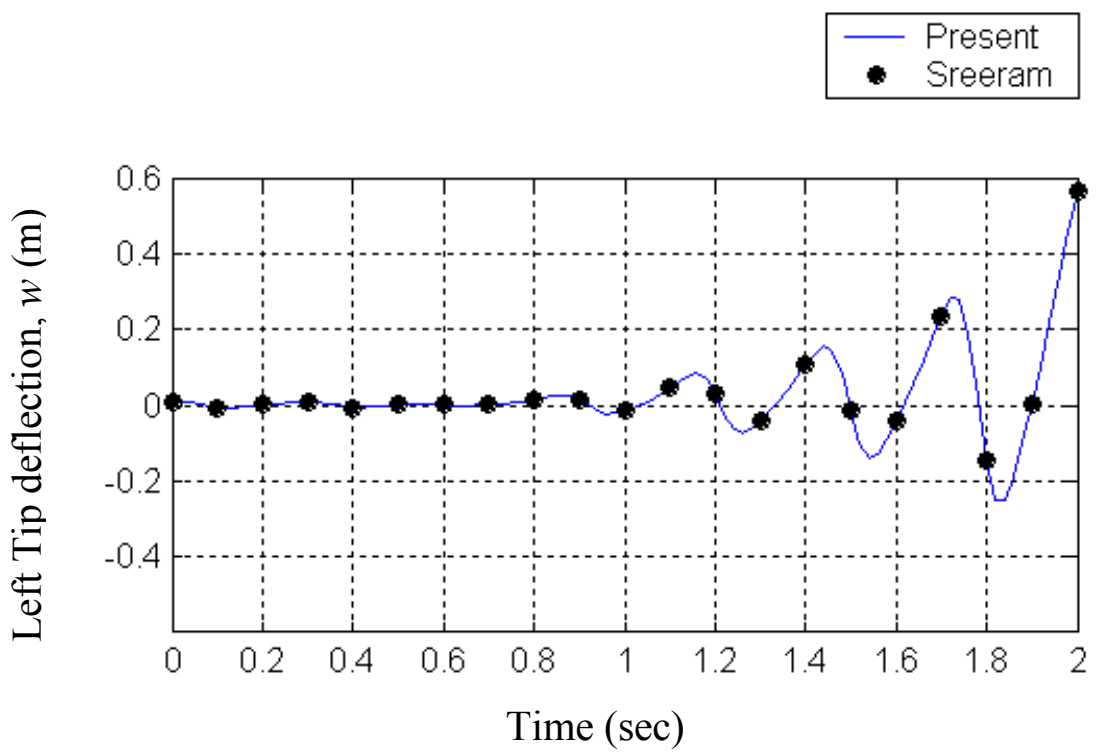

Figure 4.4 Response analysis of initially deformed isotropic moving beam with $\Omega$ $=22 \mathrm{rad} / \mathrm{sec}$

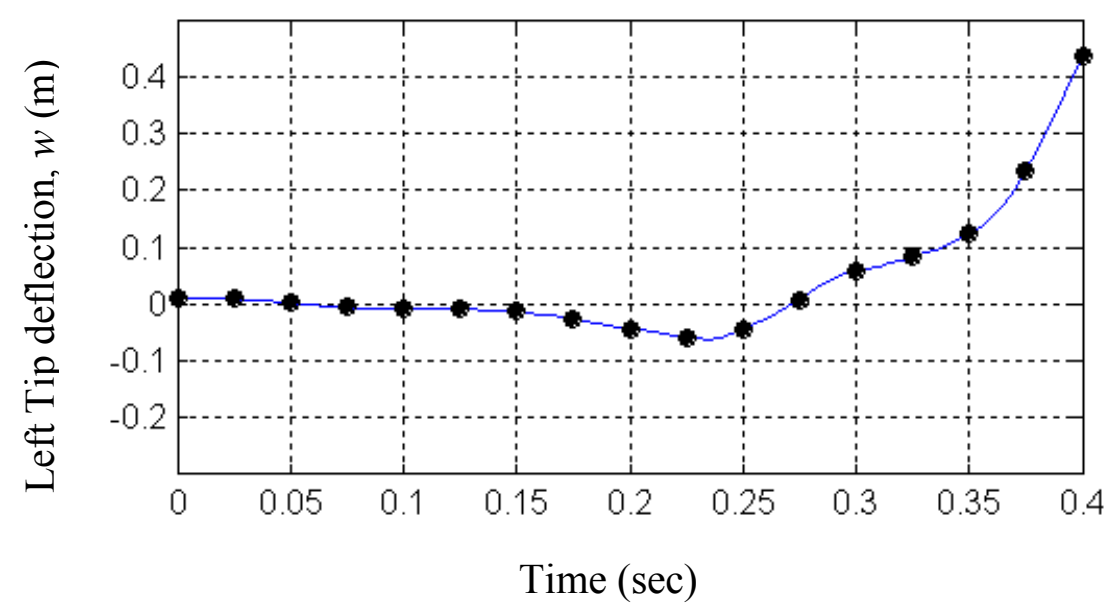

Figure 4.5 Response analysis of initially deformed isotropic moving beam with $\Omega=30 \mathrm{rad} / \mathrm{sec}$ 


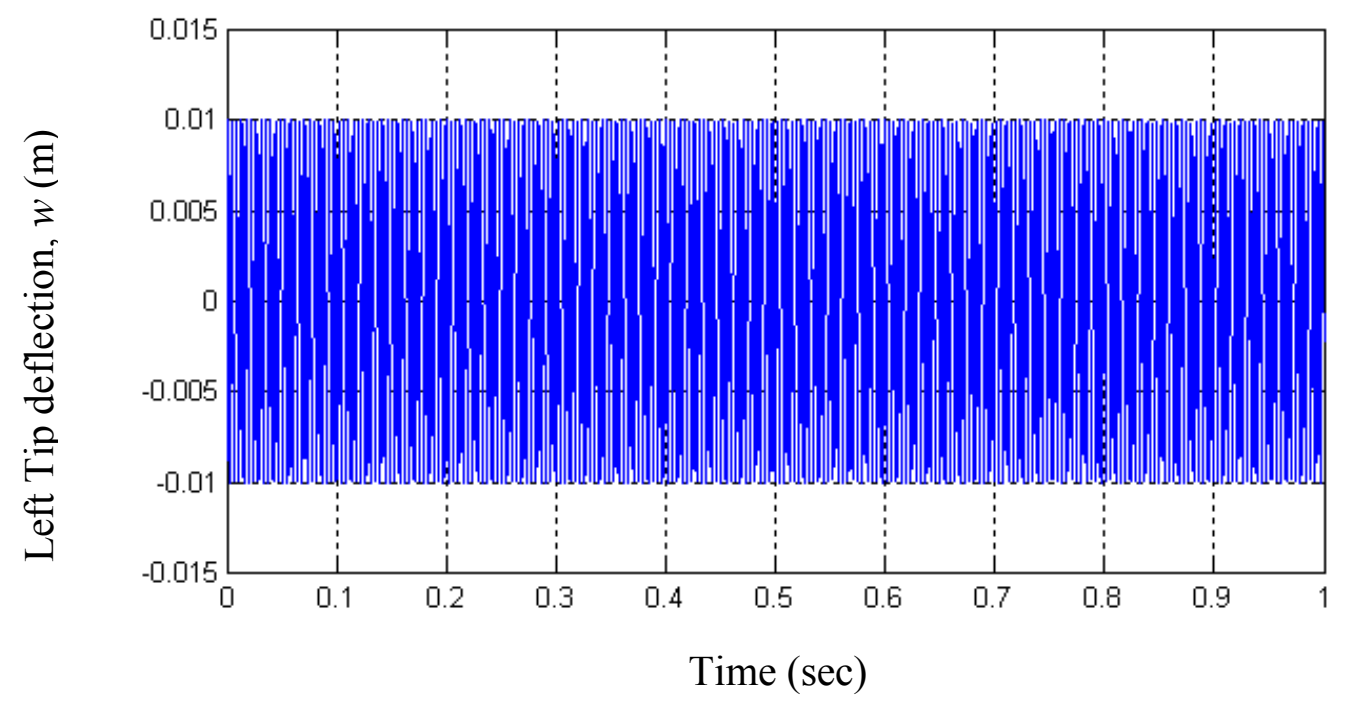

Figure 4.6 Response of initially deformed steel beam oscillating axially with $\Omega=0$ $\mathrm{rad} / \mathrm{sec}$

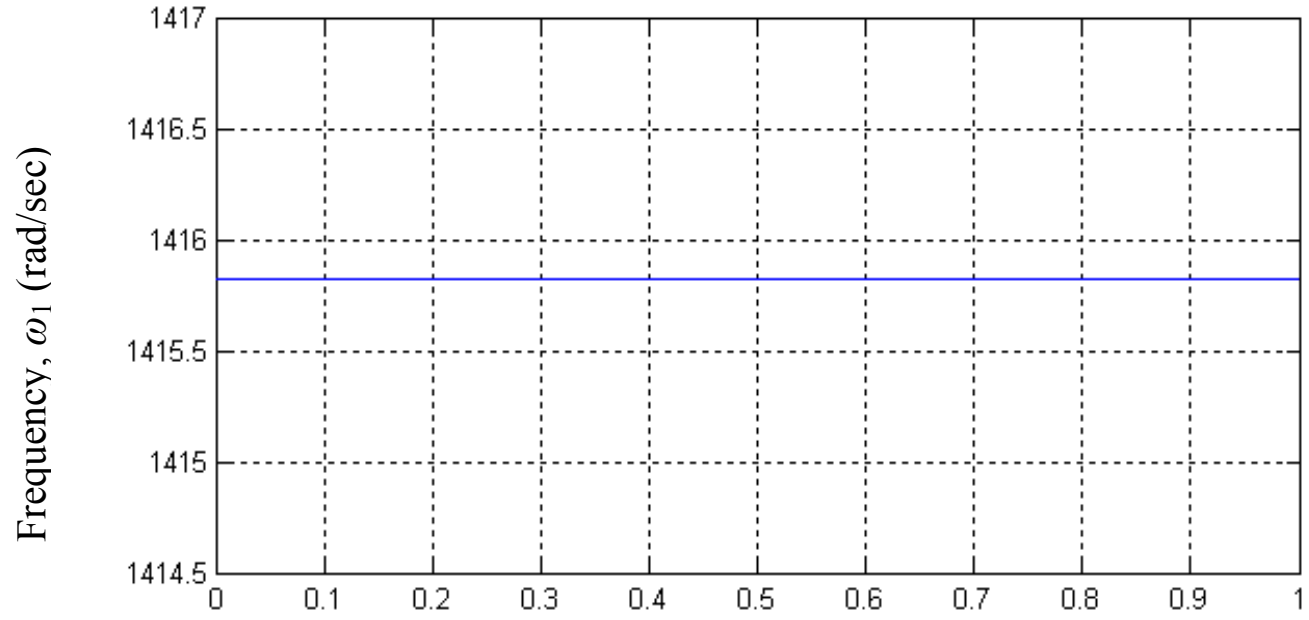

Time (sec)

Figure 4.7 First transverse natural frequency of initially deformed steel beam oscillating axially with $\Omega=0 \mathrm{rad} / \mathrm{sec}$ 


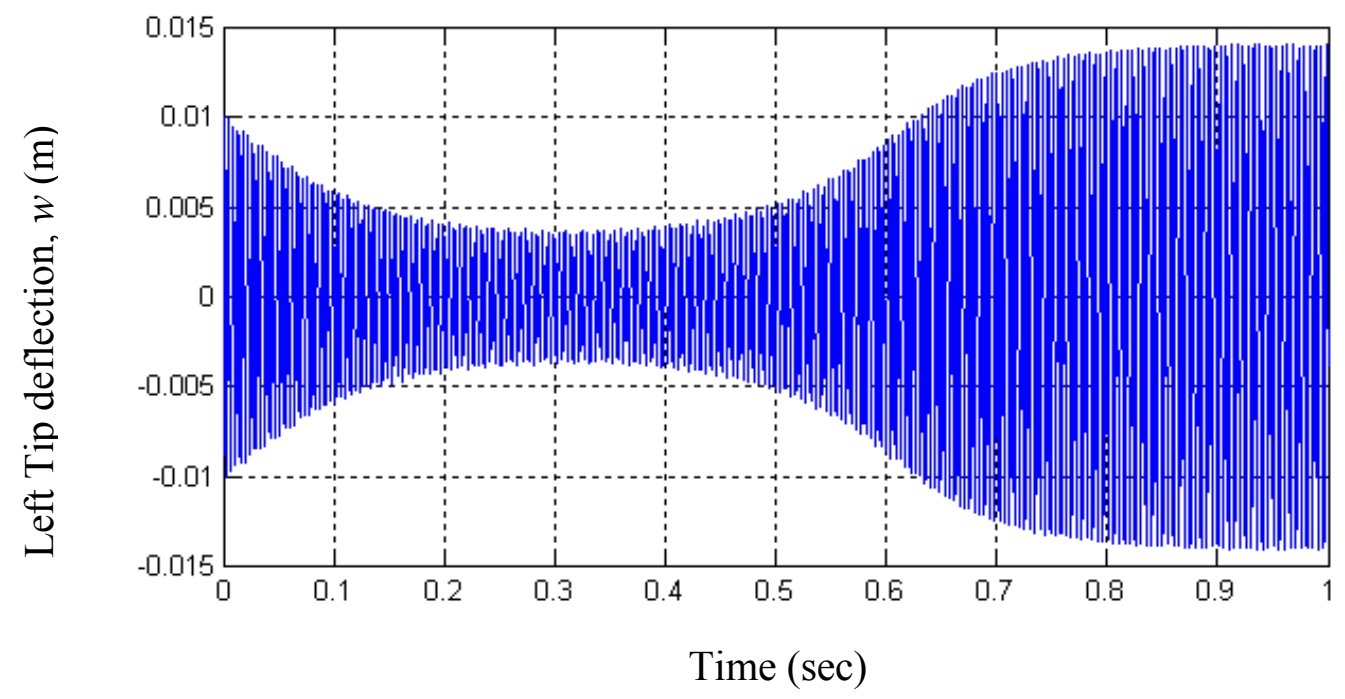

Figure 4.8 Response of initially deformed steel beam oscillating axially with $\Omega=5$ $\mathrm{rad} / \mathrm{sec}$

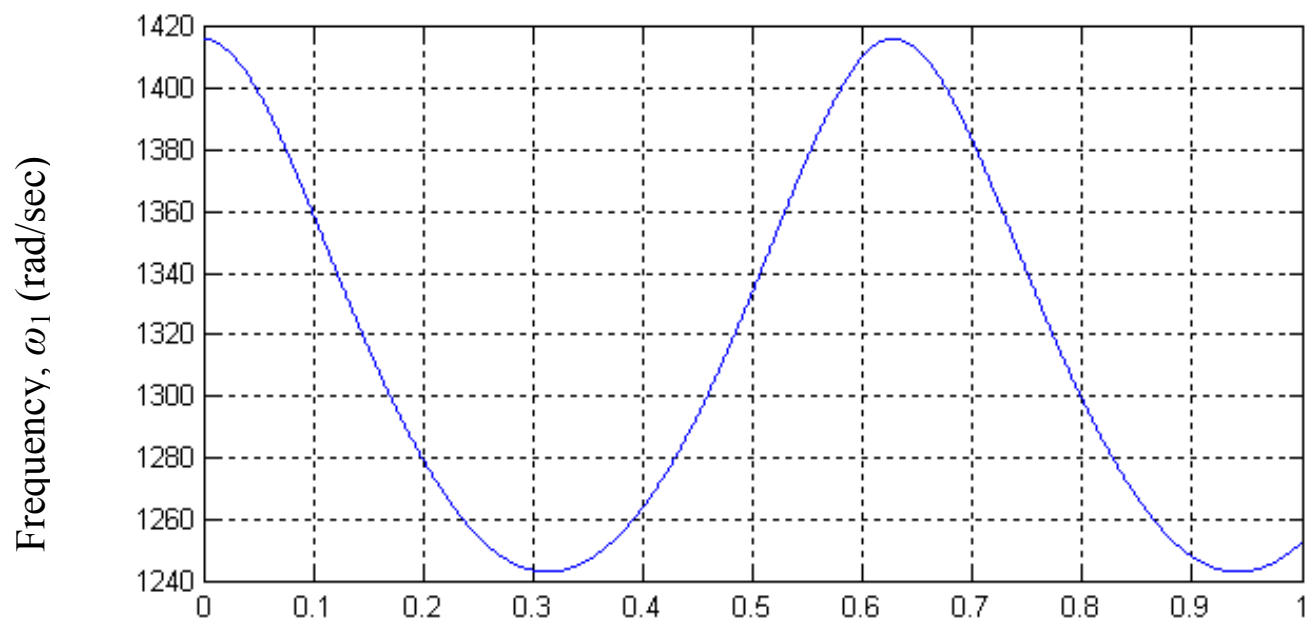

Time (sec)

Figure 4.9 First transverse natural frequency of initially deformed steel beam oscillating axially with $\Omega=5 \mathrm{rad} / \mathrm{sec}$ 


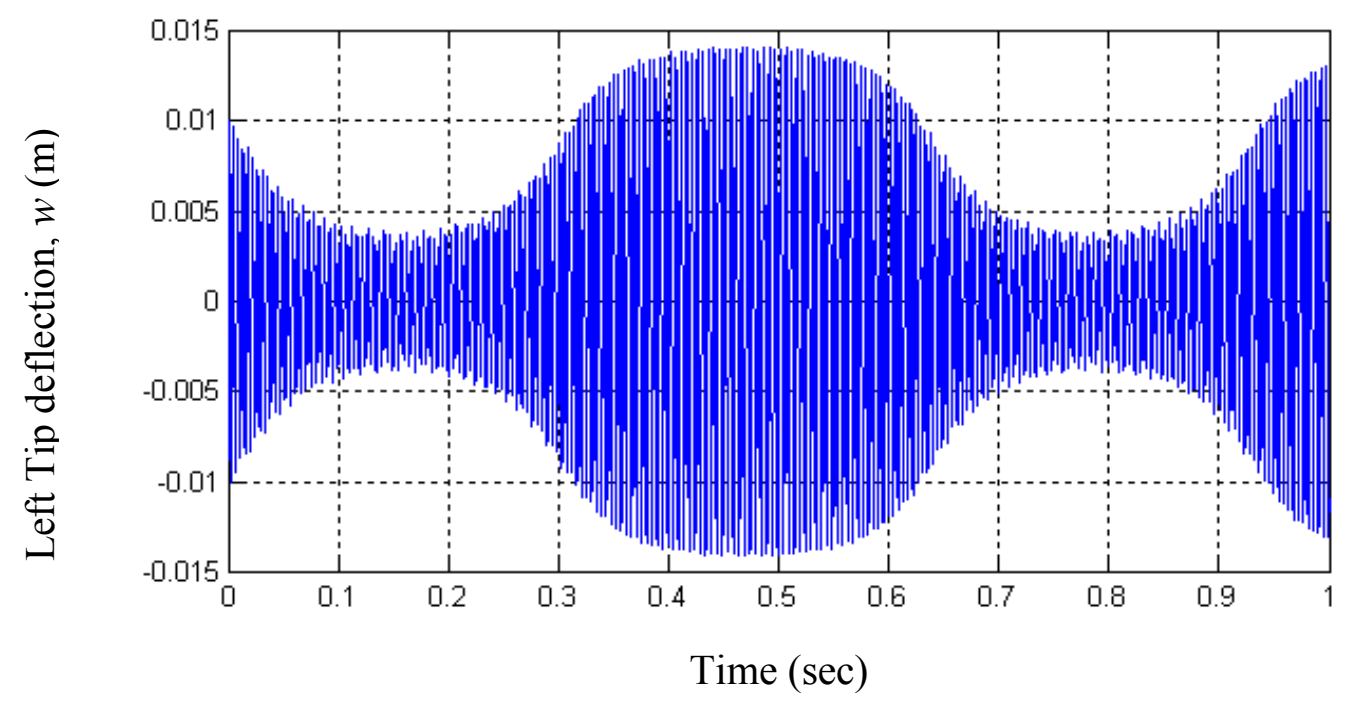

Figure 4.10 Response of initially deformed steel beam oscillating axially with $\Omega=10$ $\mathrm{rad} / \mathrm{sec}$

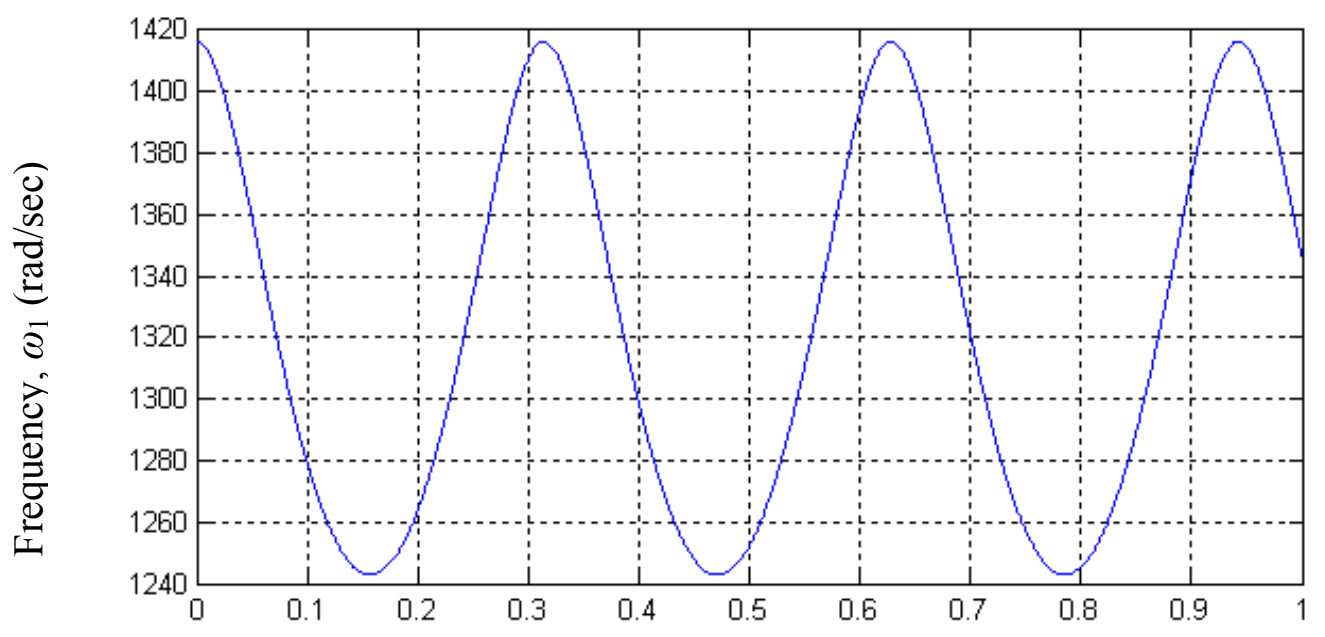

Time (sec)

Figure 4.11 First transverse natural frequency of initially deformed steel beam oscillating axially with $\Omega=10 \mathrm{rad} / \mathrm{sec}$ 


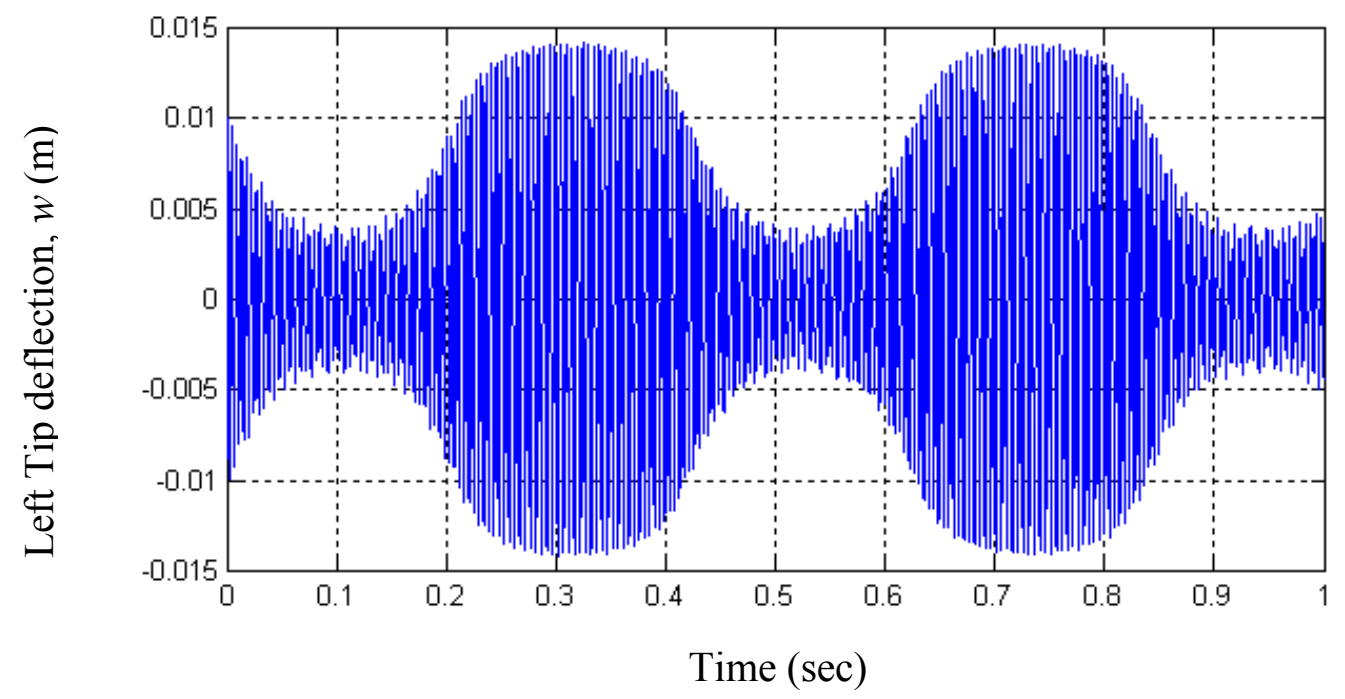

Figure 4.12 Response of initially deformed steel beam oscillating axially with $\Omega=15$ $\mathrm{rad} / \mathrm{sec}$

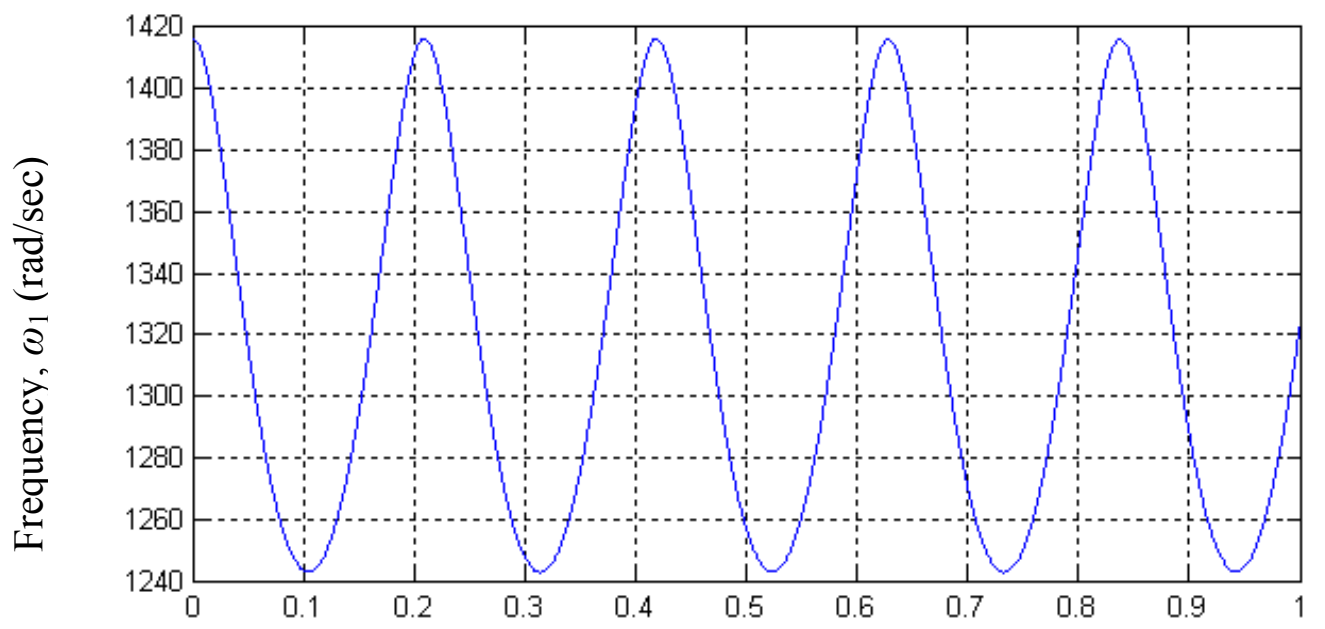

Time (sec)

Figure 4.13 First transverse natural frequency of initially deformed steel beam oscillating axially with $\Omega=15 \mathrm{rad} / \mathrm{sec}$ 


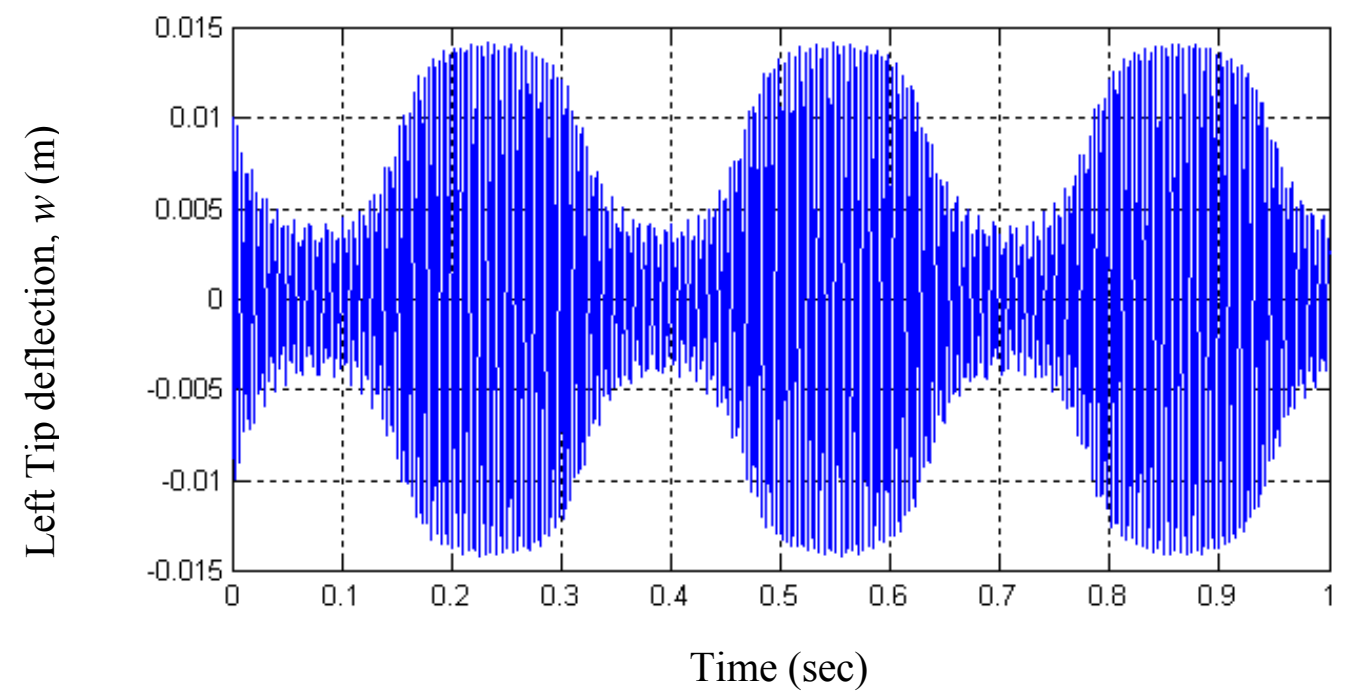

Figure 4.14 Response of initially deformed steel beam oscillating axially with $\Omega=20$ $\mathrm{rad} / \mathrm{sec}$

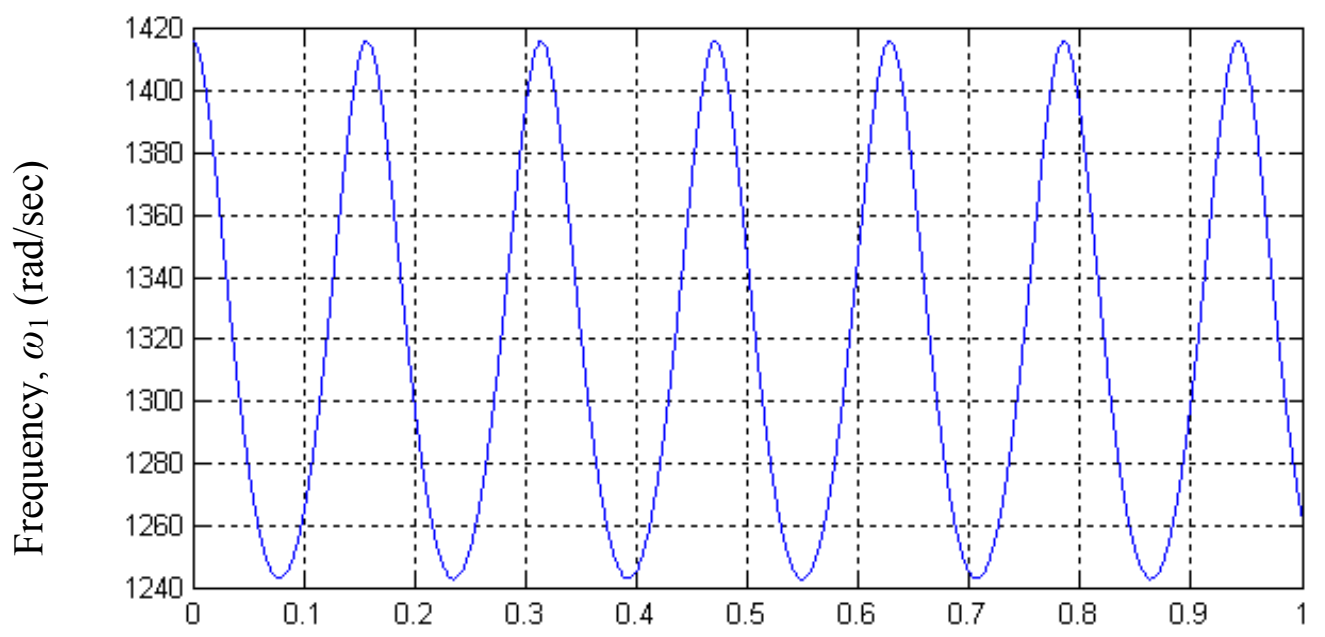

Time (sec)

Figure 4.15 First transverse natural frequency of initially deformed steel beam oscillating axially with $\Omega=20 \mathrm{rad} / \mathrm{sec}$ 


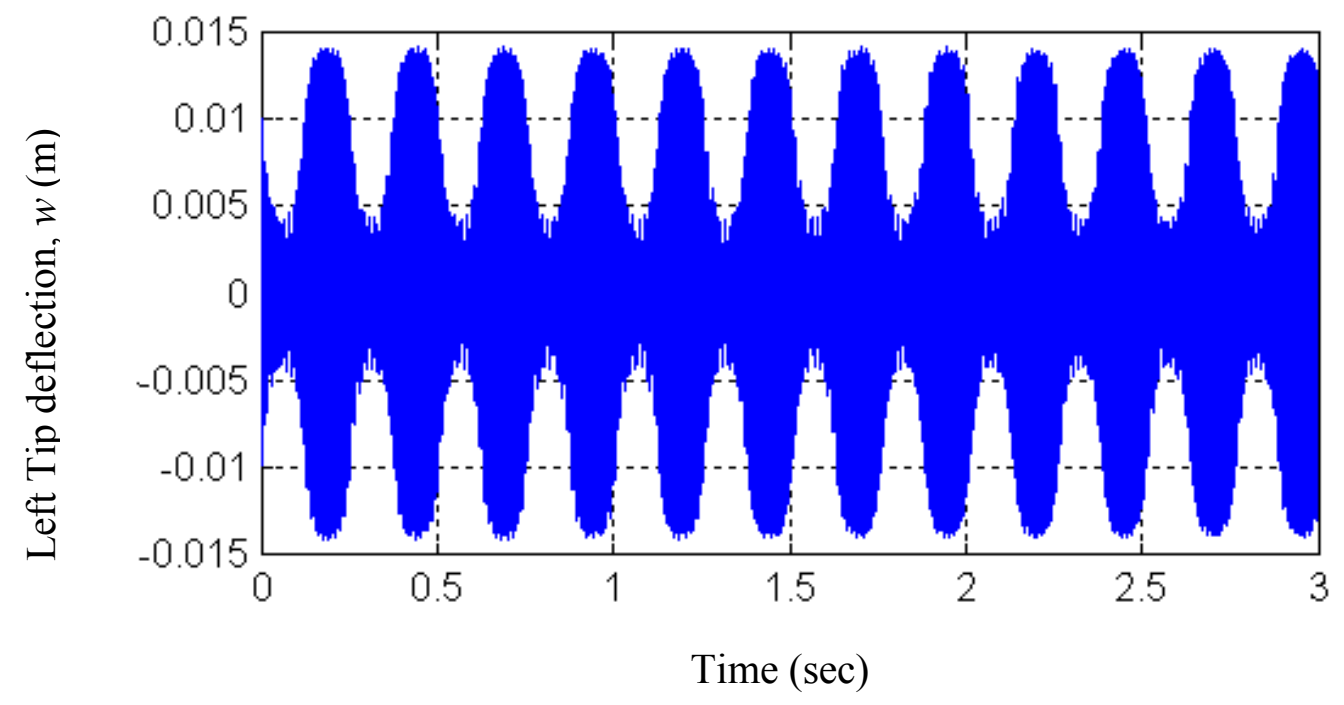

Figure 4.16 Response of initially deformed steel beam oscillating axially with $\Omega=\mathbf{2 5}$ $\mathrm{rad} / \mathrm{sec}$

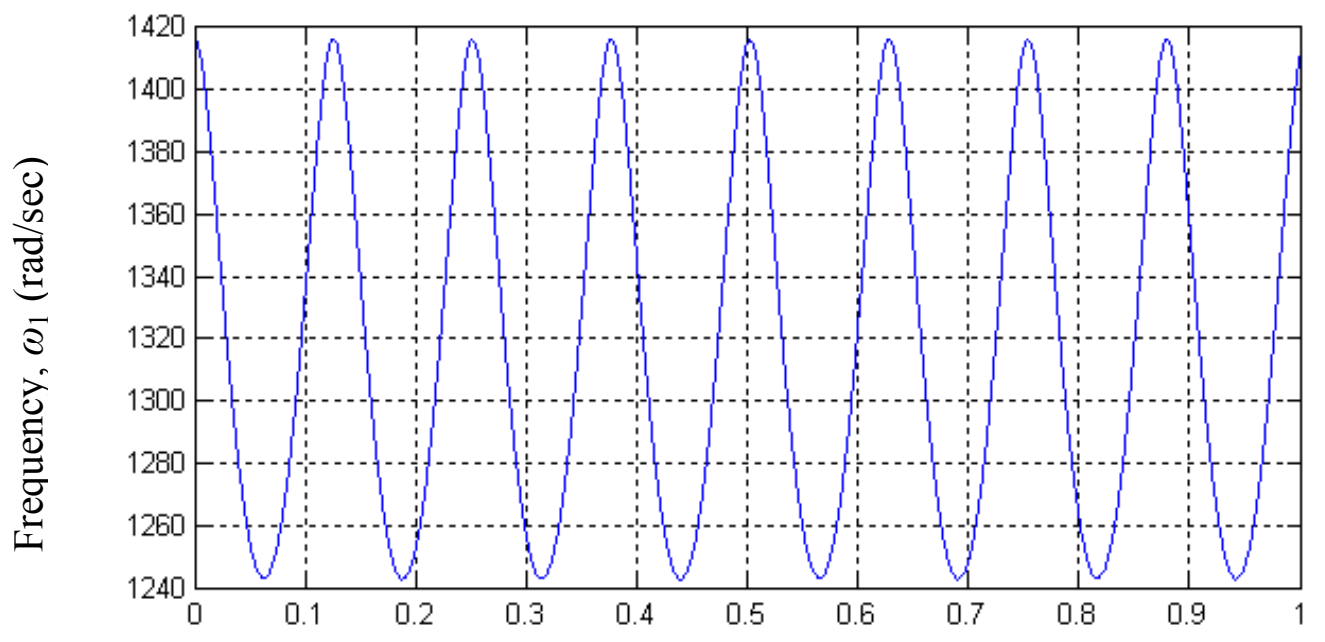

Time (sec)

Figure 4.17 First transverse natural frequency of initially deformed steel beam oscillating axially with $\Omega=25 \mathrm{rad} / \mathrm{sec}$ 
Table 4.2 for the range of $\Omega$ considered. Since the initial tip deflection is $0.01 \mathrm{~m}$, we can calculate the magnification factor by which the tip deflection is enhanced. The values listed in the last column of the table indicate that the magnification factor shows a gradual upward trend with increasing $\Omega$. The factor ranges from 1.40 to 1.42 . Thus the axial motion induces the tip deflection to go up by about $40 \%$.

\subsection{Validation of program using HSDT}

The results presented so far validate the use of Lagrange multiplier method to establish the boundary conditions and the use of time integration method to solve the second order differential equation describing the moving beam. In this section the validation of stiffness matrix and the inertia matrix (indirectly) formulated using HSDT are carried out. The formulation based on HSDT is validated for nonmoving laminated beams. A simply supported composite beam subjected to uniform loading is considered. The aspect ratio of the beam $(L / h)$ is taken as 100 . The graphite-epoxy material properties used are,

$$
\begin{array}{ll}
E_{1}=144.8 \mathrm{GPa} & G_{23}=3.448 \mathrm{GPa} \\
E_{2}=9.653 \mathrm{GPa} & \rho=1389.227 \mathrm{Kg} / \mathrm{m}^{3} \\
G_{12}=G_{13}=4.137 \mathrm{GPa} & v_{12}=0.3
\end{array}
$$

The non-dimensional central deflections of the beam for different lay-up configurations are calculated using the present program. The beam is divided into 4 higher-order ( $h-p$ version) elements. The non-dimensional central deflection is defined as,

$$
\bar{w}=w_{\max } \frac{E_{2} I}{p_{0} L^{4}} * 100
$$


where $I$ is the moment of inertia and $p_{0}$ is the intensity of the uniformly distributed load. The results are compared (Table 4.3) to the corresponding results of Shi et al. (1998) and excellent agreement is seen. Shi et al. divided the beam into sixteen $h$-version elements.

\begin{tabular}{|l|l|l|}
\hline Lay-up & Shi (1998) & Present \\
\hline$[0]$ & 0.08677 & 0.08701 \\
\hline$[0 / 90 / 90 / 0]$ & 0.09827 & 0.09837 \\
\hline
\end{tabular}

Table 4.3 Transverse deflections of composite beam obtained using HSDT

The results produced so far validate only the stiffness matrix. To validate the inertia matrix, vibration behavior of the composite beam is studied and the results are compared with those available in literature. The natural frequencies and the mode shapes describe the vibration behavior of the beam. The non-dimensional fundamental frequency of a beam with fixed end conditions is determined. The same properties used in the previous problem are used here except for the $L / h$ ratio, which is taken to be 15 . The nondimensional fundamental frequency is given by,

$$
\bar{\omega}=\omega L^{2} \sqrt{\frac{\gamma}{E_{1} h^{2}}}
$$

where $\omega$ is the fundamental frequency and $\gamma$ is the mass per unit length.

The frequencies are compared with the corresponding results of Kadivar and Mohebpour (1997). Kadivar and Mohebpour (1997) have presented the non-dimensional fundamental frequencies for the finite element model using FSDT. Therefore, in the present case frequencies are calculated by reducing HSDT to FSDT by setting $c_{1}$ to be zero in Eq. (2.6). The frequencies for finite element model using FSDT are also calculated based on a separate computer program written for FSDT alone. The FSDT 
uses a shear correction factor in the formulation. So, while reducing the HSDT to FSDT a shear correction factor of $K=5 / 6$ is used in Eq. (2.31). As seen from Table 4.3, the present results of columns 3 and 4 are in excellent agreement with that of Kadivar and Mohebpour (column 2). This table also shows the natural frequencies obtained based on HSDT (column 5). Its is clearly seen that the HSDT does alter the natural frequencies in an appreciable way, particularly for $\theta$ values of 45 degree or less of the angle-ply laminate $[\underline{+} \theta]_{\mathrm{s}}$.

\begin{tabular}{|l|l|l|l|l|}
\hline $\begin{array}{l}\text { Lay-up } \\
\text { Configuration }\end{array}$ & $\begin{array}{l}\text { Kadivar and } \\
\text { Mohebpour } \\
(1997)(F S D T)\end{array}$ & $\begin{array}{l}\text { FSDT } \\
\text { (present) }\end{array}$ & $\begin{array}{l}\text { HSDT with } \\
\mathrm{C}_{1}=0 \text { (present) }\end{array}$ & $\begin{array}{l}\text { HSDT } \\
\text { (present) }\end{array}$ \\
\hline $0 /-0 /-0 / 0$ & 4.8629 & 4.8684 & 4.8684 & 4.3897 \\
\hline $15 /-15 /-15 / 15$ & 4.0082 & 3.9845 & 3.9845 & 3.4145 \\
\hline $30 /-30 /-30 / 30$ & 2.8762 & 2.8604 & 2.8604 & 2.4134 \\
\hline $45 /-45 /-45 / 45$ & 1.9330 & 1.9304 & 1.9304 & 1.7867 \\
\hline $60 /-60 /-60 / 60$ & 1.6290 & 1.6291 & 1.6291 & 1.5975 \\
\hline $75 /-75 /-75 / 75$ & 1.6063 & 1.6065 & 1.6065 & 1.5805 \\
\hline $90 /-90 /-90 / 90$ & 1.6161 & 1.6163 & 1.6163 & 1.5900 \\
\hline
\end{tabular}

Table 4.4 Non-dimensional fundamental frequencies of composite beam

\subsection{Composite Moving Beam}

The time response of a composite moving beam is presented in this section. The results are generated based on the three different plate theories mentioned earlier, namely CLPT, FSDT, and HSDT. For each of the three theories, a separate computer program has been written in MATLAB to produce the desired results. A parametric study in the 
form of two different axial frequencies and three different laminate configurations is carried out. Since shear effects are more pronounced in a short beam, an aspect ratio $(L / h)$ of 16.67 is considered.

The geometric properties of the overhang beam under consideration (See Figure 4.1) are as follows:

Beam length $(L)=1.0 \mathrm{~m}$

Beam height $(h)=0.06 \mathrm{~m}$

Beam width $(b)=0.05 \mathrm{~m}$

Distance between the supports $(d)=0.25 L$

Initial distance of the left end of the beam from the first support, $S_{1},\left(X_{0}\right)=0.375 \mathrm{~L}$

Amplitude of axial rigid body motion $(A)=0.05 \mathrm{~m}$

Frequency of the axial rigid body motion $(\Omega)=10,20 \mathrm{rad} / \mathrm{sec}$

Lay-ups considered: $[0 / \pm 45],[0 / \pm 20],[0 / \pm 45]_{S}$

The ply properties of the graphite-epoxy composite are:

$$
\begin{array}{ll}
E_{1}=144.8 \mathrm{GPa} & G_{23}=3.448 \mathrm{GPa} \\
E_{2}=9.653 \mathrm{GPa} & \rho=1389.227 \mathrm{Kg} / \mathrm{m}^{3} \\
G_{12}=G_{13}=4.137 \mathrm{GPa} & v_{12}=0.3
\end{array}
$$

\subsubsection{Moving Beam Simulation using CLPT}

At $t=0$, the beam is given an initial deformed shape, $w(x, 0)$, corresponding to the first mode shape in bending of the overhang beam normalized such that the left and right end deflections are $0.01 \mathrm{~m}$. Then the beam is subjected to an axial motion

$$
X_{A}(t)=-X_{0}+A \operatorname{Sin}(\Omega t)
$$


and the time response is obtained by solving the finite element equations of motion, Eq. (3.29), using the Wilson Theta method with a theta value of 1.4. The computer program was run starting with a time step, $\Delta t=2.5 \times 10^{-4} \mathrm{sec}$ for a time interval of few seconds and the results became numerically divergent at about $1 \mathrm{sec}$. The $\Delta t$ was gradually reduced to figure out the optimum time step that would prevent numerical divergence for the first two seconds or so of the time interval. This optimal time step was found to be $2.5 \times 10^{-6} \mathrm{sec}$. The results are plotted for a time interval of $1 \mathrm{sec}$. The transverse deflection of the left end of the beam for the laminate $[0 / \pm 45]$ at an axial frequency of $10 \mathrm{rad} / \mathrm{sec}$ over a time interval of 0 to $1 \mathrm{sec}$ is shown in Figure 4.18. Just as observed in the steel beam, the response of the composite beam shows a beat-like phenomenon. The larger beat period is equal to $T_{\Omega}$ of $0.628 \mathrm{sec}$, as was the case earlier. The study of the steel beam in Section 4.2 has indicated that the response frequency is not equal to but closely related to the first transverse natural frequency and a similar phenomenon is present in the composite case also.

As mentioned earlier, the midplane axial deflection $u$ is set to zero at the left end of the beam to avoid rigid-body movement. Thus the maximum axial deflection is expected at the right end of the beam and this is plotted as a function of time in Figure 4.19. The axial deflection also shows a beat behavior, although to a much lesser extent. The amplitudes of axial deflection are one order lower than that of the transverse deflection.

The response of the same laminate $[0 / \pm 45]$ at the axial frequency of $20 \mathrm{rad} / \mathrm{sec}$ is presented next. The transverse deflection $w$ for this case is seen in Figure 4.20. Again, the response shows a beat-like behavior but with a smaller beat period due to the higher axial 
frequency. The transverse deflection of the laminate $[0 / \pm 20]$ for $\Omega=10$ and $20 \mathrm{rad} / \mathrm{sec}$ are shown in Figures 4.21 and 4.22, respectively. The behavior is similar to that of the $[0 / \pm 45]$ laminate except with a higher frequency of response. This is to be expected since the $[0 / \pm 20]$ laminate is stiffer. The transverse deflection response of a symmetric laminate, $[0 / \pm 45]_{\mathrm{s}}$ with an axial frequency of $20 \mathrm{rad} / \mathrm{sec}$ is presented in Figure 4.23 . The response indicates that this laminate is stiffer than the other two laminates. The axial deflection of this beam can be seen in Figure 4.24 and as expected this deflection is essentially zero due to the absence of bending/in-plane coupling in the symmetric laminate.

\begin{tabular}{|c|l|l|c|}
\hline Lay-up & $\Omega(\mathrm{rad} / \mathrm{sec})$ & $\begin{array}{c}w(\max ) \\
(\mathrm{m})\end{array}$ & $\begin{array}{c}\text { Magnification Factor } \\
\frac{(w)_{\max }}{(w)_{t=0}}\end{array}$ \\
\hline $0 / 45 /-45$ & 10 & 0.01400 & 1.4000 \\
\hline $0 / 45 /-45$ & 20 & 0.01412 & 1.4120 \\
\hline $0 / 20 /-20$ & 10 & 0.01433 & 1.4330 \\
\hline $0 / 20 /-20$ & 20 & 0.01430 & 1.4300 \\
\hline$[0 / 45 /-45]_{\mathrm{s}}$ & 20 & 0.01412 & 1.4120 \\
\hline
\end{tabular}

Table 4.5 Maximum tip deflections of Beams based on CLPT

The magnification factors for the five cases of CLPT are presented in Table 4.5. In each case the magnification factor is slightly higher than 1.4. The instantaneous natural frequency as a function of time for the five cases of CLPT composite beam is presented in Figures 4.25-4.29. As observed in the case of steel beams, the effect of the axial motion is to reduce the instantaneous natural frequency. Table 4.6 summarizes the results of the CLPT composite beam study. The beat period is equal to $T_{\Omega}$, the period of axial oscillation. The instantaneous natural frequency, $\omega_{1}$, and the response frequency, $\omega$, do 
not vary with $\Omega$ just as observed in the steel beam. The response frequency $\omega$, falls within the range of the instantaneous natural frequency $\omega_{1}$.

\begin{tabular}{|c|c|c|c|c|c|c|}
\hline $\begin{array}{c}\text { CLPT } \\
\text { Lay Up }\end{array}$ & \multicolumn{2}{|c|}{$\Omega$ (forcing - axial) } & $T_{\Omega}$ & \multicolumn{2}{|c|}{$\omega_{1}$ (natural) } & \multirow{2}{*}{$\begin{array}{c}\omega \text { (response) } \\
\mathrm{Hz}\end{array}$} \\
\cline { 2 - 6 } & $\mathrm{rad} / \mathrm{sec}$ & $\mathrm{Hz}$ & $\mathrm{sec}$ & $\mathrm{rad} / \mathrm{sec}$ & $\mathrm{Hz}$ & \\
\hline $0 / 45 /-45$ & 10 & 1.59 & 0.628 & $1199-1368$ & $190.83-217.72$ & 203 \\
\hline $0 / 45 /-45$ & 20 & 3.18 & 0.314 & $1199-1368$ & $190.83-217.72$ & 203 \\
\hline $0 / 20 /-20$ & 10 & 1.59 & 0.628 & $2004-2320$ & $318.95-369.24$ & 341 \\
\hline $0 / 20 /-20$ & 20 & 3.18 & 0.314 & $2004-2320$ & $318.95-369.24$ & 341 \\
\hline$[0 / 45 /-45]_{\mathrm{s}}$ & 20 & 3.18 & 0.314 & $2163-2463$ & $344.25-391.99$ & 364 \\
\hline
\end{tabular}

Table 4.6 Instantaneous first natural frequency of beams based on CLPT 
Lay-up $=0 / 45 /-45$

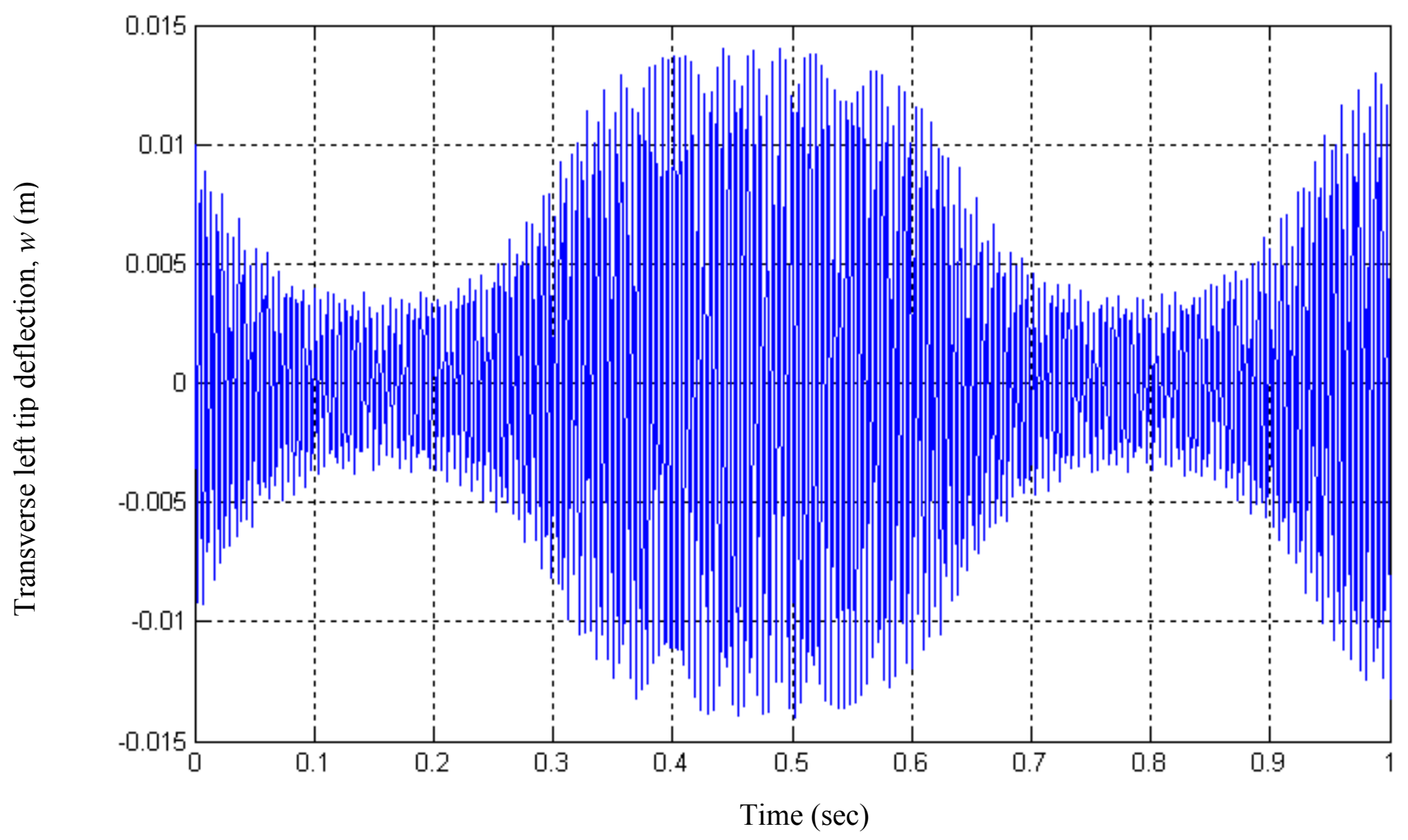

Figure 4.18 Transverse left tip deflection $w$, CLPT, $\Omega=10 \mathrm{rad} / \mathrm{sec}$ 
Lay-up $=0 / 45 /-45$

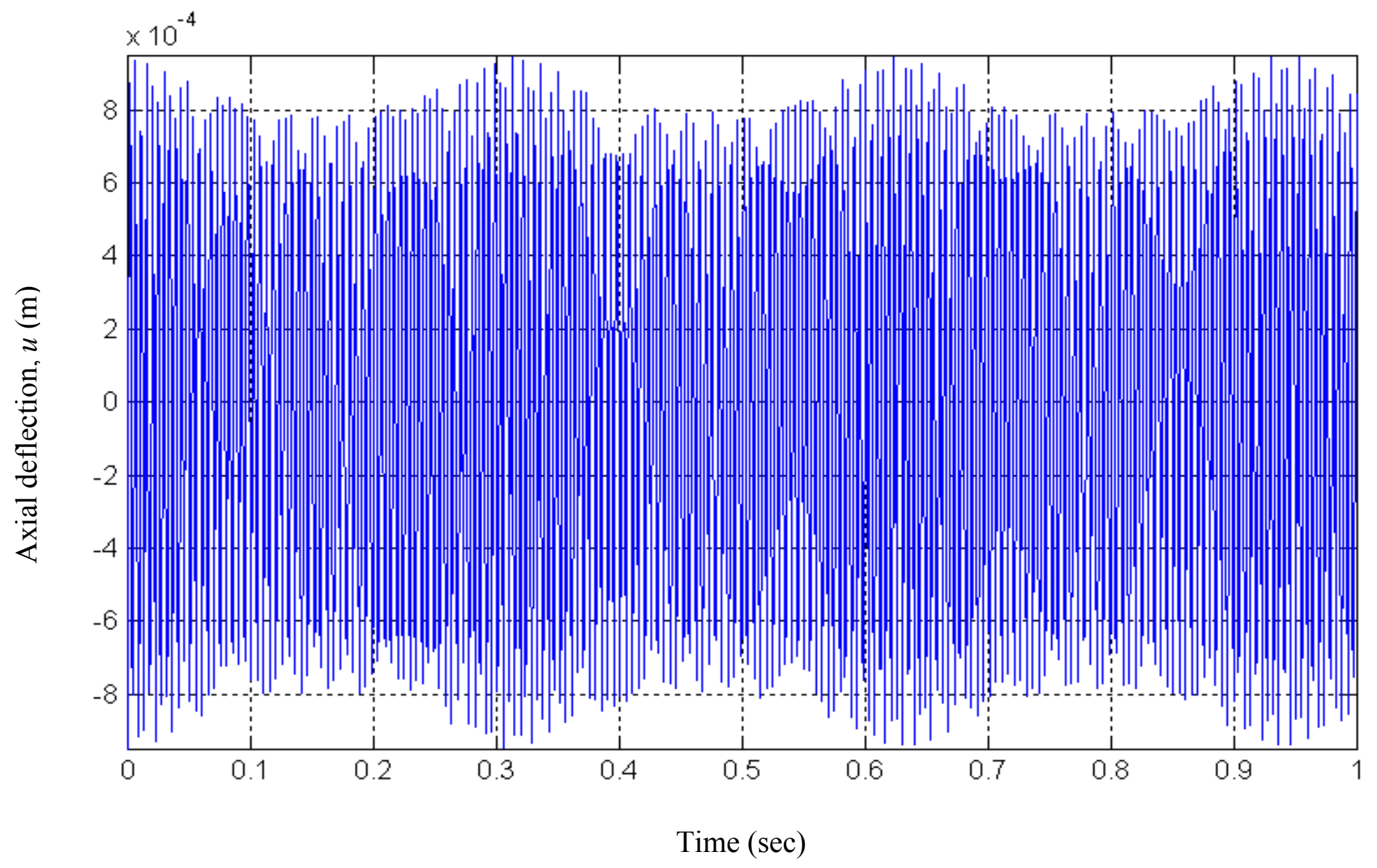

Figure 4.19 Axial right tip deflection $u$, CLPT, $\Omega=10 \mathrm{rad} / \mathrm{sec}$ 
Lay-up $=0 / 45 /-45$

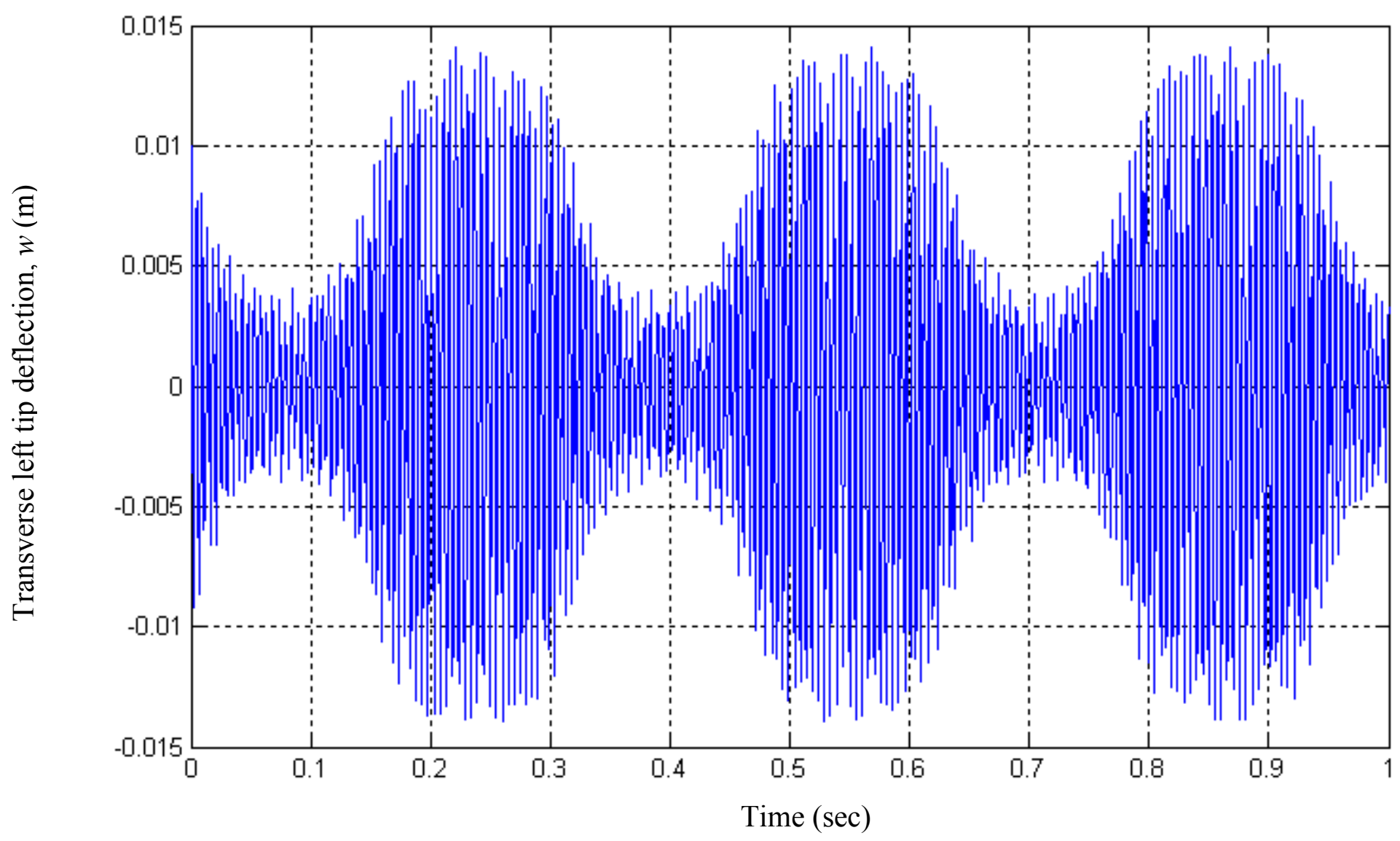

Figure 4.20 Transverse left tip deflection $w$, CLPT, $\Omega=20 \mathrm{rad} / \mathrm{sec}$ 
Lay-up $=0 / 20 /-20$

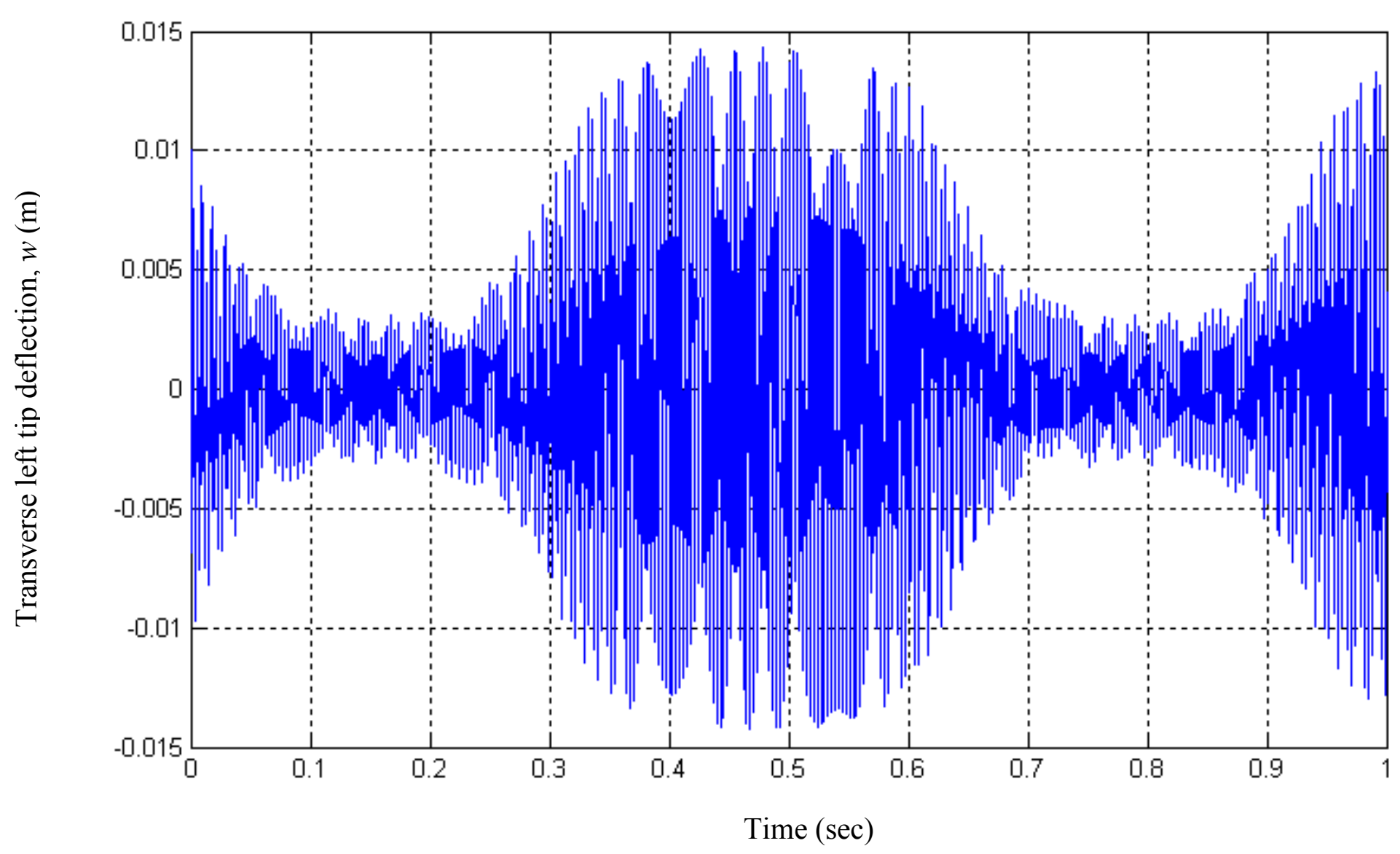

Figure 4.21 Transverse left tip deflection $w$, CLPT, $\Omega=10 \mathrm{rad} / \mathrm{sec}$ 
Lay-up $=0 / 20 /-20$

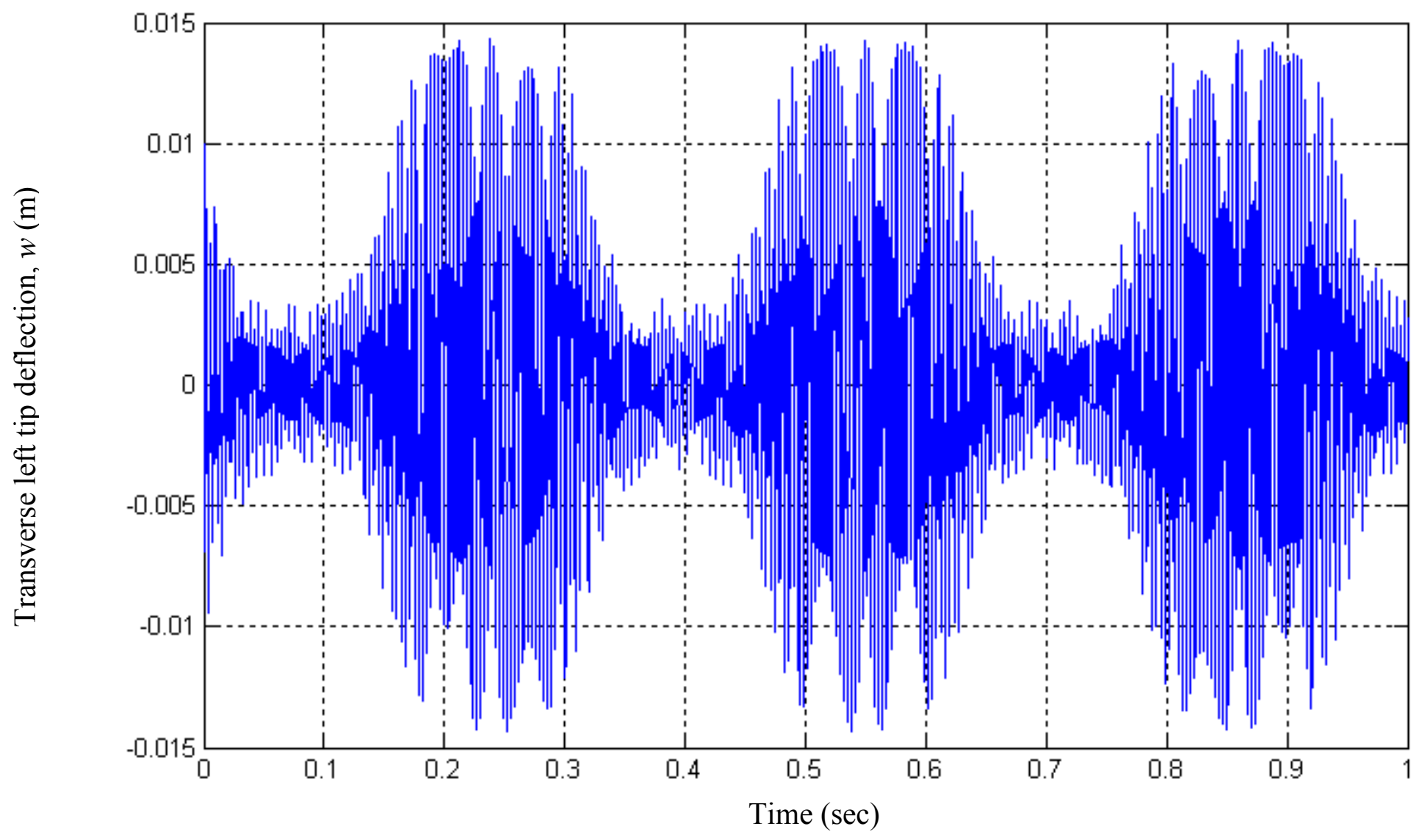

Figure 4.22 Transverse left tip deflection $w$, CLPT, $\Omega=20 \mathrm{rad} / \mathrm{sec}$ 
Lay-up $=0 / 45 /-45 /-45 / 45 / 0$

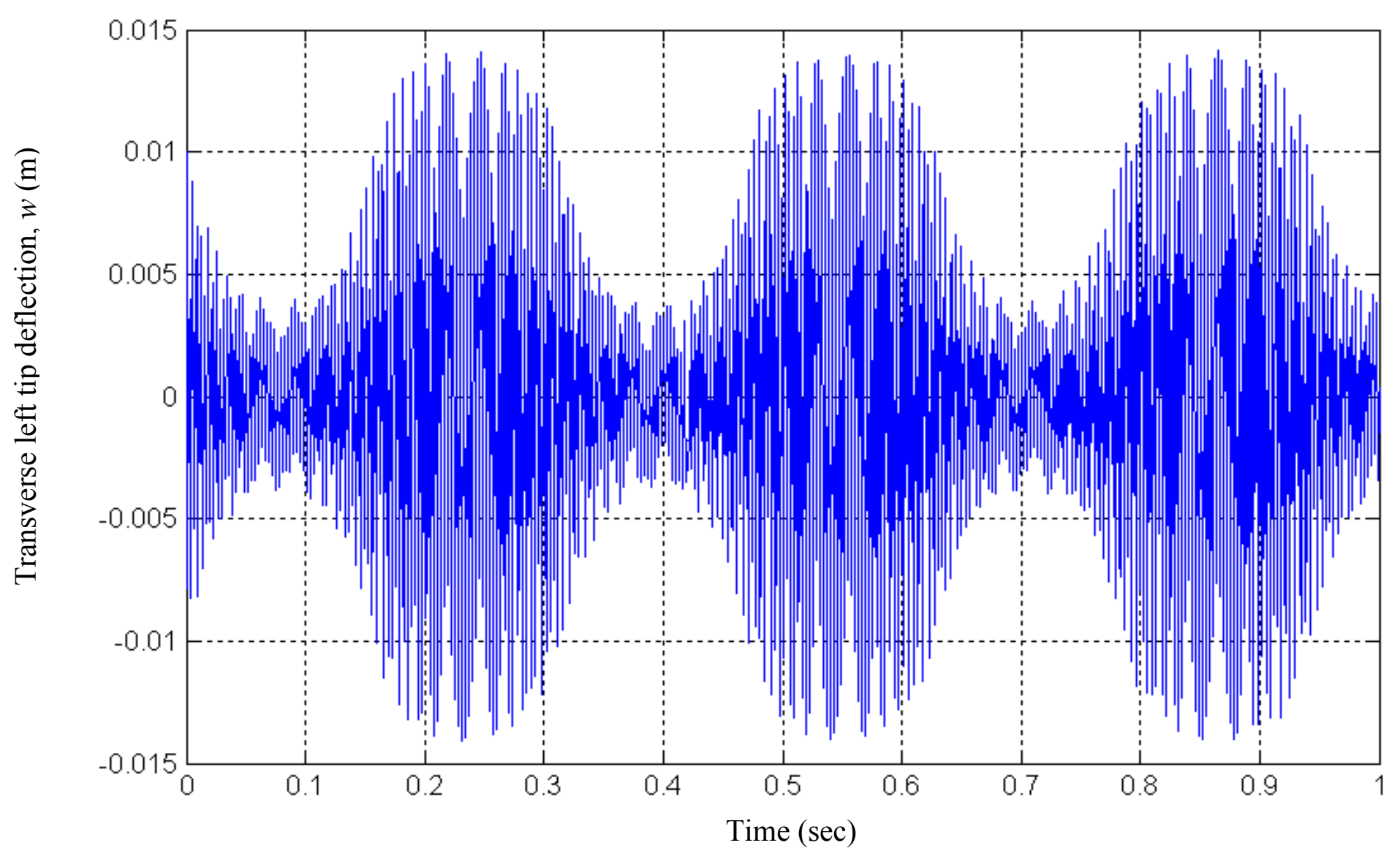

Figure 4.23 Transverse left tip deflection $w$, CLPT, $\Omega=20 \mathrm{rad} / \mathrm{sec}$ 
Lay-up $=0 / 45 /-45 /-45 / 45 / 0$

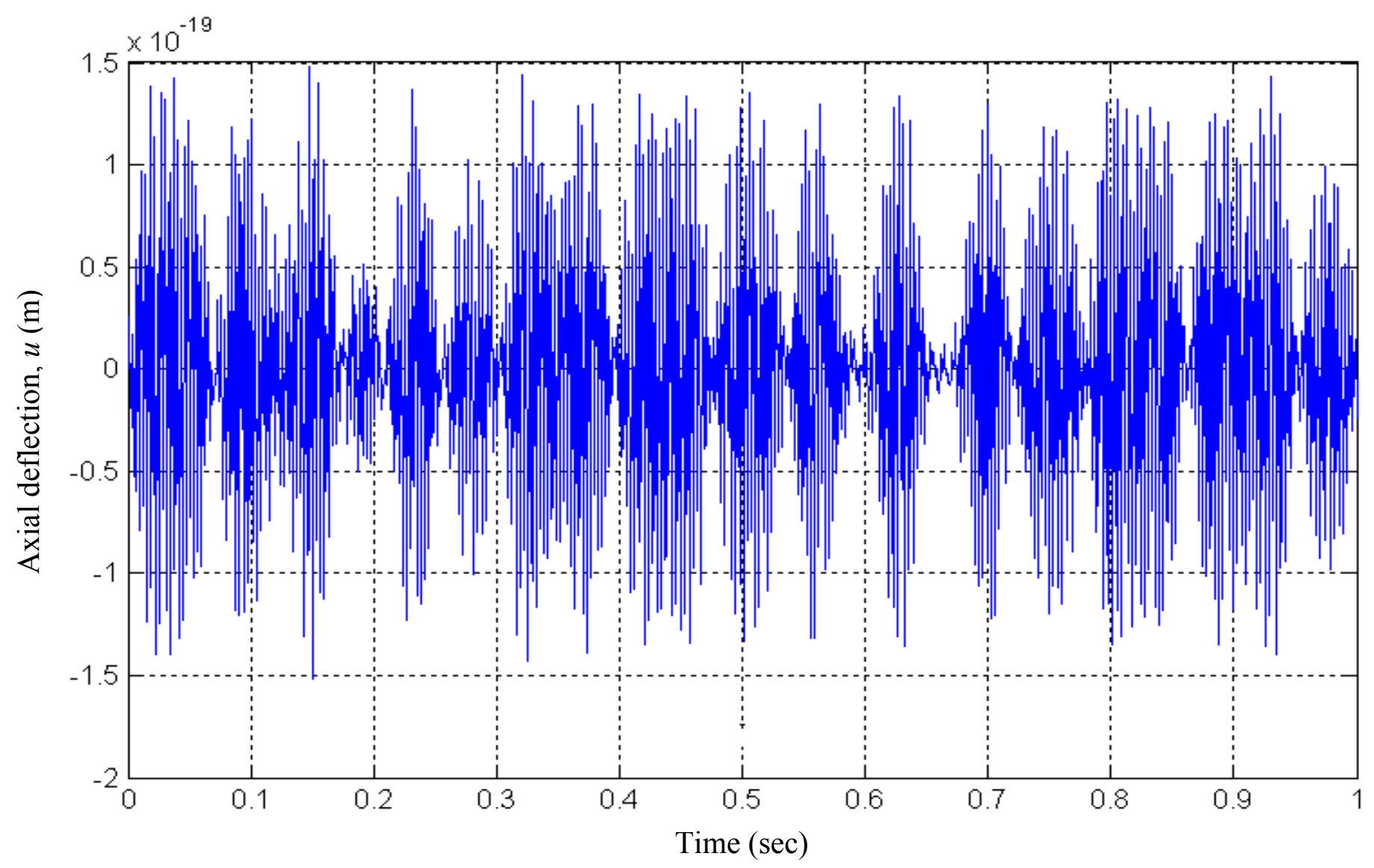

Figure 4.24 Axial right tip deflection $u, C L P T, \Omega=20 \mathrm{rad} / \mathrm{sec}$ 


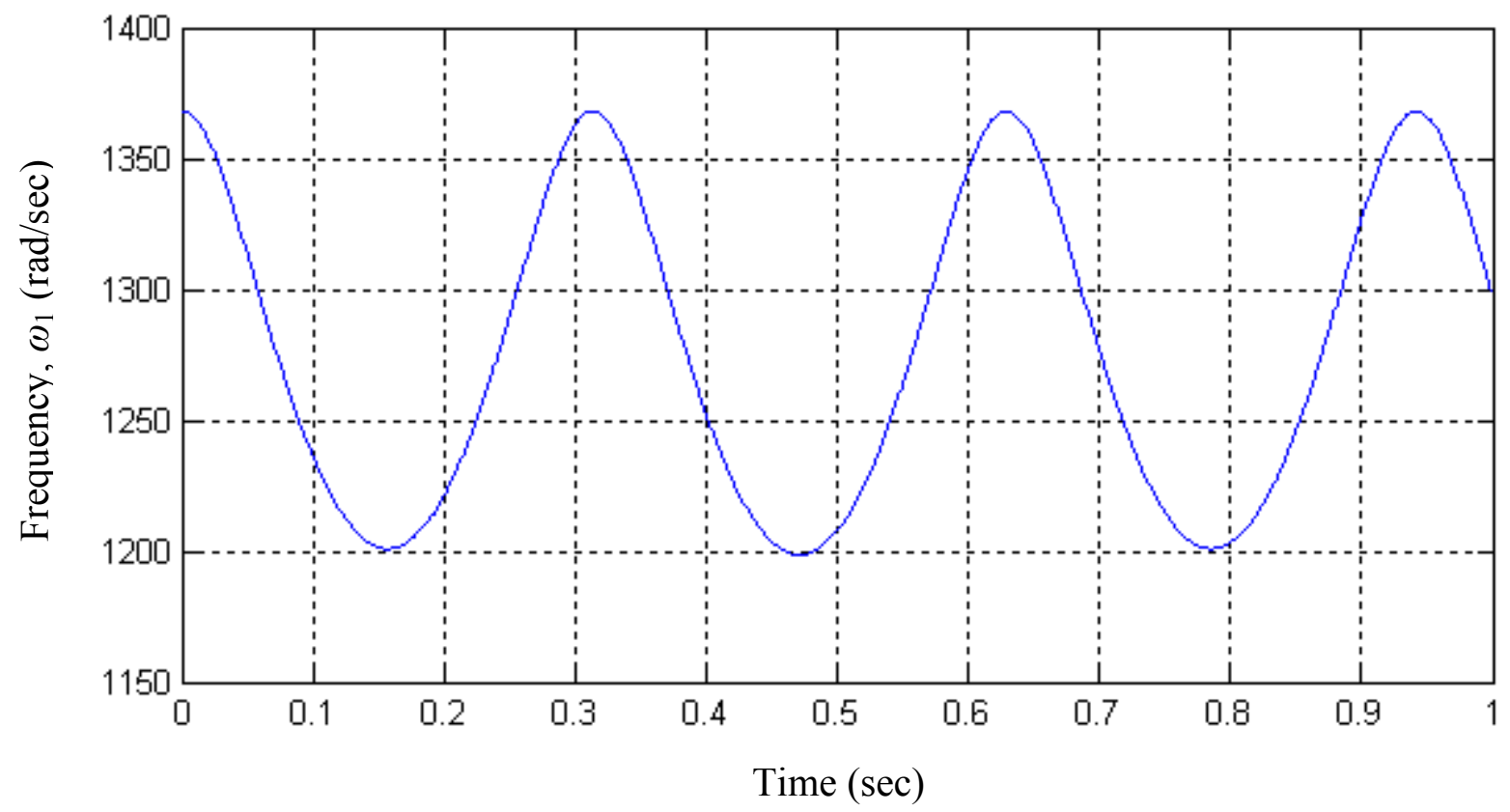

Figure 4.25 First transverse natural frequency $\omega_{1}$, of $[0 / \pm 45]$, CLPT, $\Omega=10 \mathrm{rad} / \mathrm{sec}$

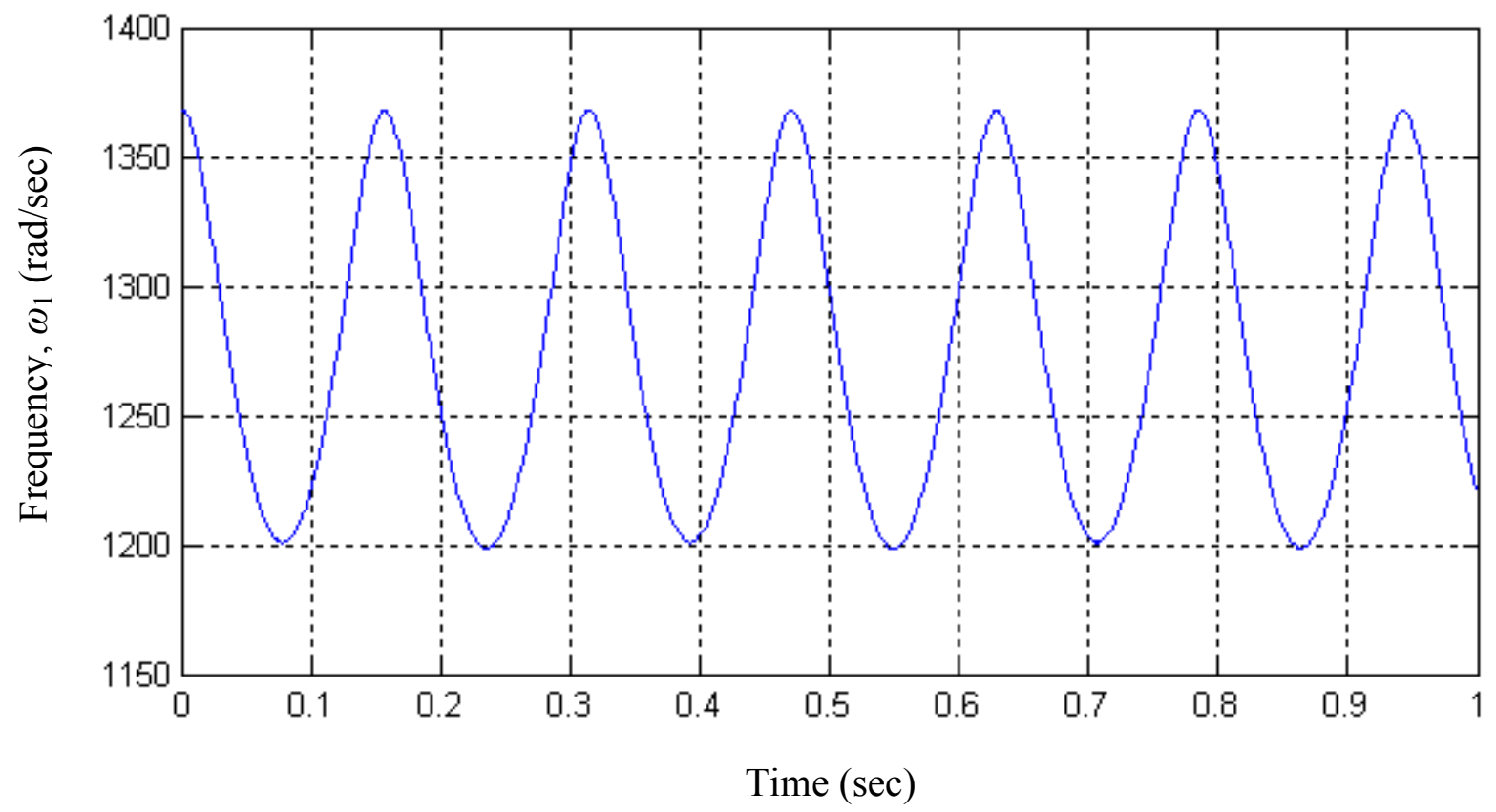

Figure 4.26 First transverse natural frequency $\omega_{1}$, of $[0 / \pm 45]$, CLPT, $\Omega=20 \mathrm{rad} / \mathrm{sec}$ 


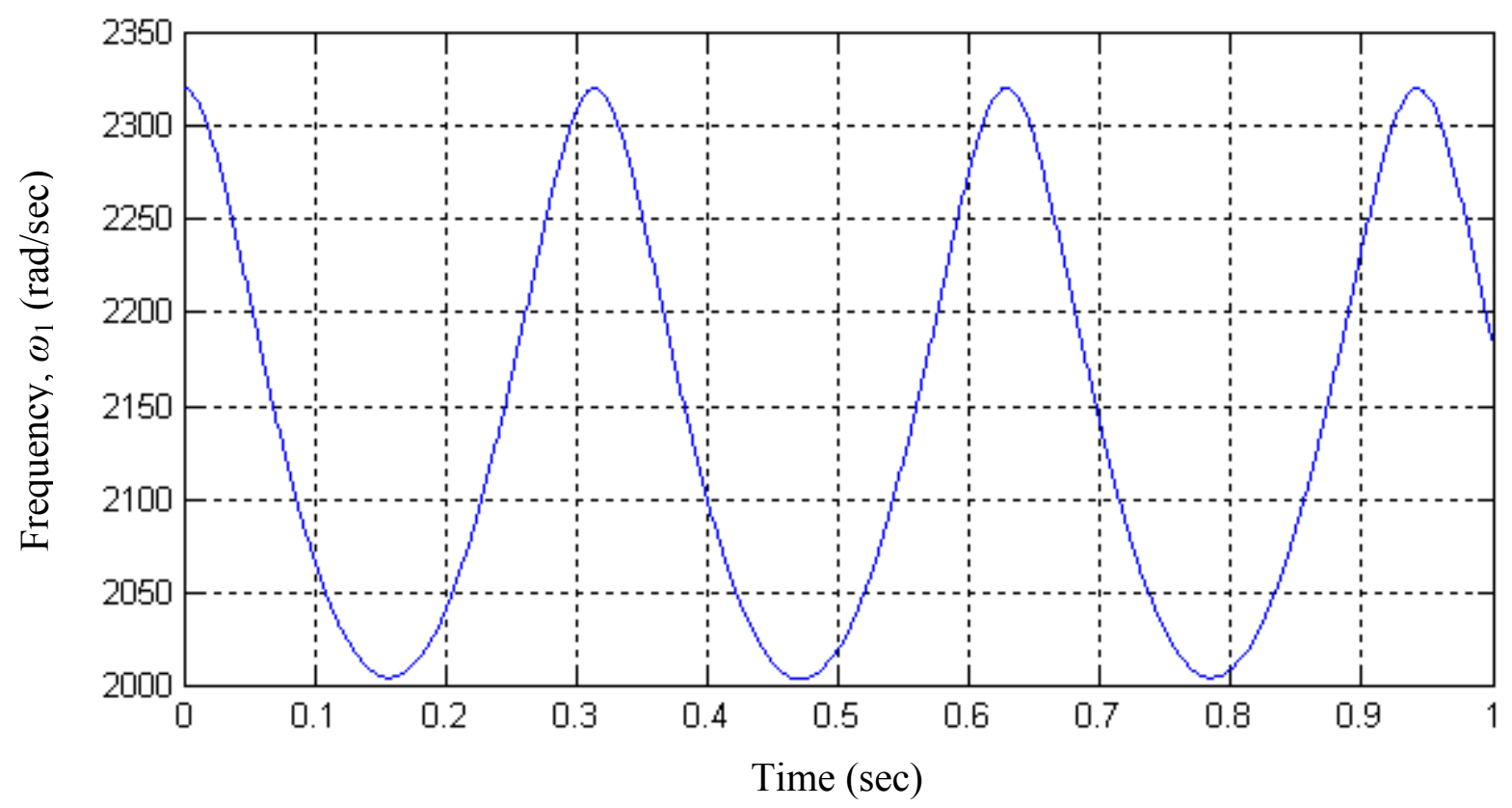

Figure 4.27 First transverse natural frequency $\omega_{1}$, of $[0 / \underline{20}]$, CLPT, $\Omega=10 \mathrm{rad} / \mathrm{sec}$

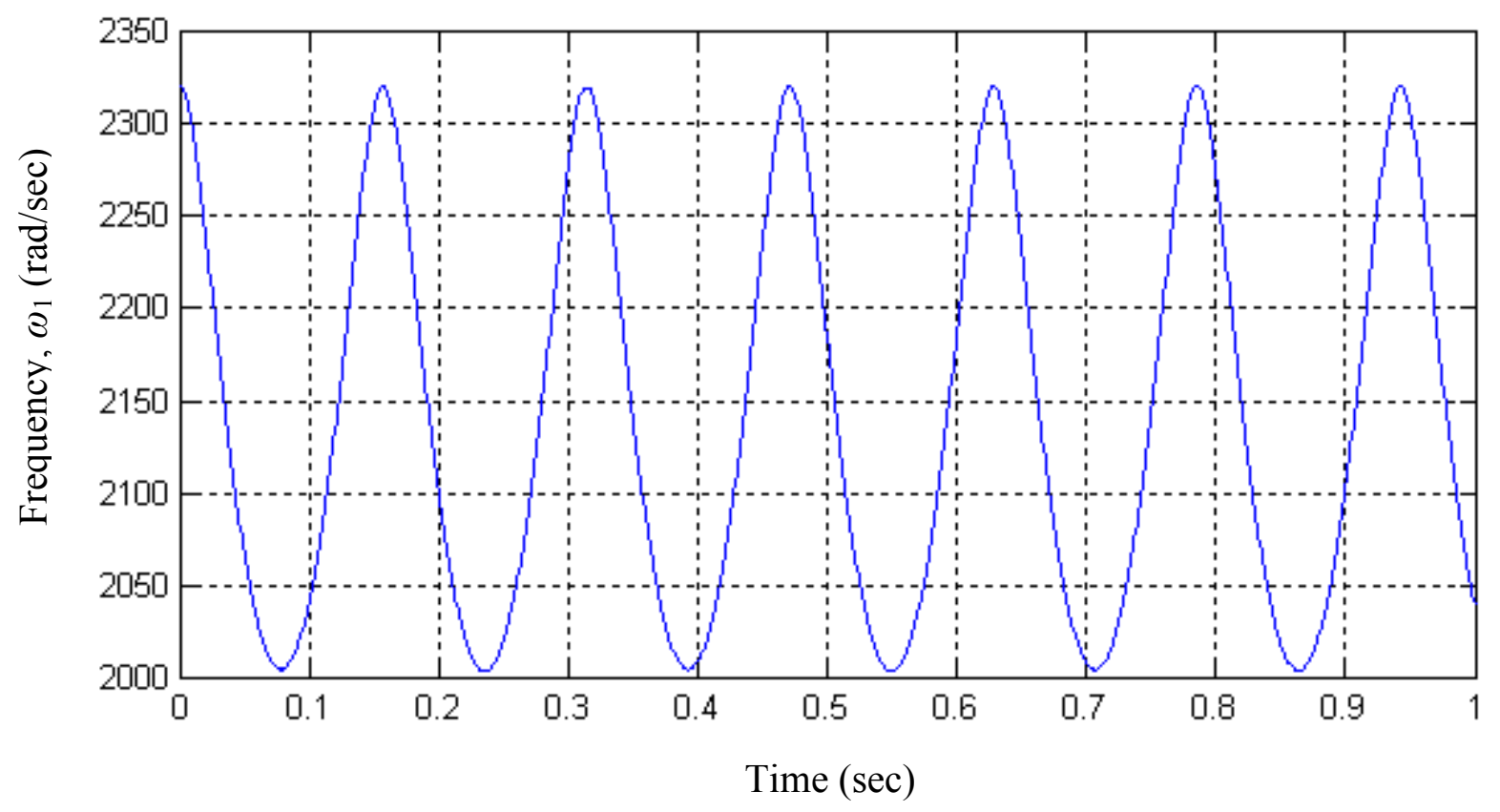

Figure 4.28 First transverse natural frequency $\omega_{1}$, of $[0 / \pm 20], C L P T, \Omega=20 \mathrm{rad} / \mathrm{sec}$ 


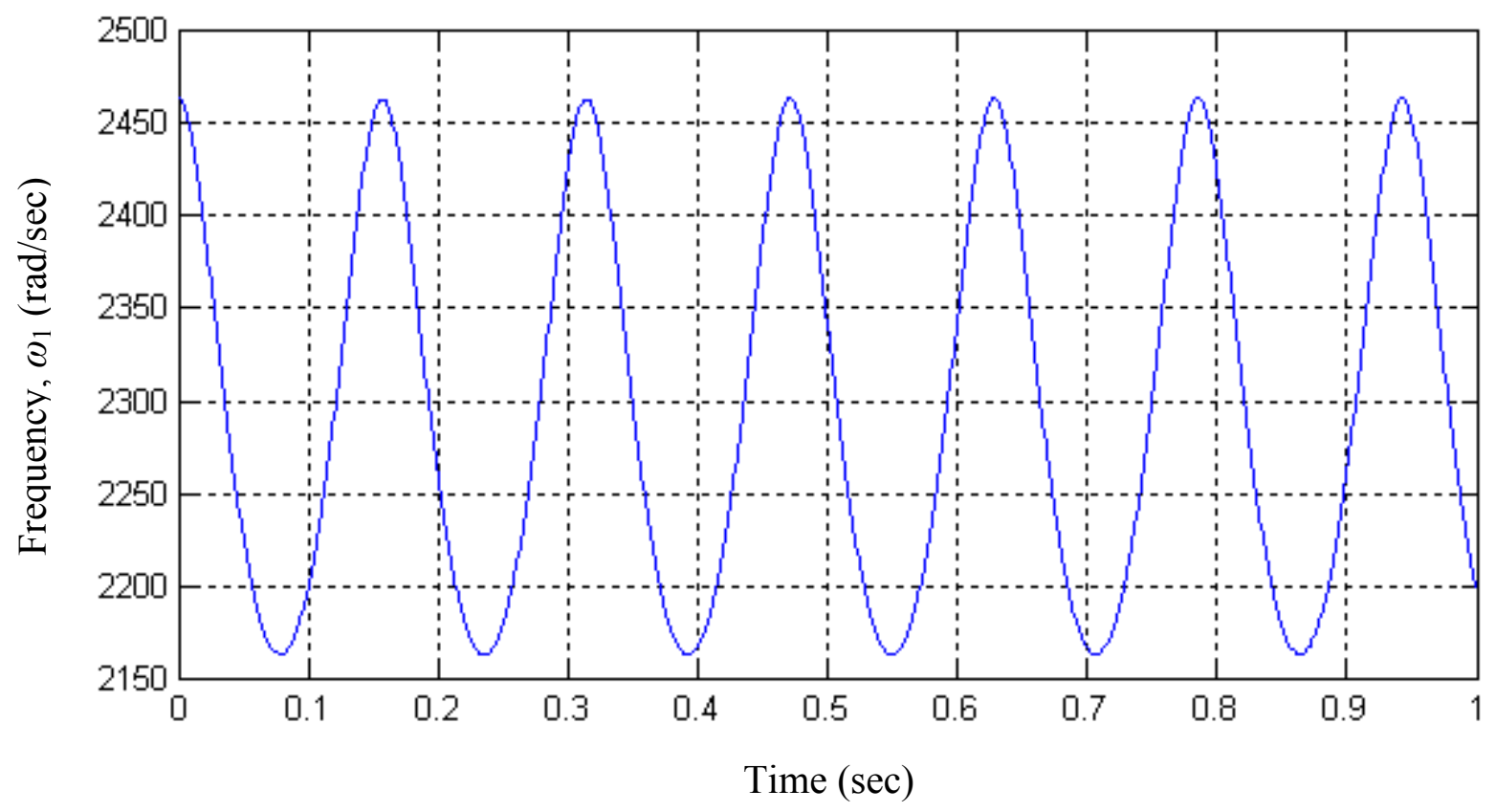

Figure 4.29 First transverse natural frequency $\omega_{1}$, of $[0 / \pm 45]_{\mathrm{s}}$ CLPT, $\Omega=10 \mathrm{rad} / \mathrm{sec}$ 


\subsubsection{Moving Beam Simulation using FSDT}

In this section the response of composite moving beams based on FSDT is presented. To compare the results with CLPT, beams with same properties and lay-up configuration as that of the CLPT are used. The finite element based on FSDT has more number of degrees of freedom (29), than that of CLPT (22 d.o.f); therefore it takes more time for the program to be executed. The computer program for this case is run for a time interval of $1 \mathrm{sec}$. The axial deflection at the left end is fixed to avoid rigid body motion as in the case of CLPT. The first mode shape of $w_{b}$ of a symmetrically placed overhang beam is taken as the initial shape. The shape is normalized such that the tip deflections are $0.01 \mathrm{~m}$.

The only dependent variables in the CLPT case were $u$ and $w$. In the case of FSDT, the dependent variables are $u, w_{b}, w_{s}, \gamma$, and $w_{b}{ }^{y}$. The results of selected variables are presented for the same five run cases as that of CLPT. The transverse deflection $w_{b}$ for the laminate $[0 / \pm 45]$ for $\Omega=10 \mathrm{rad} / \mathrm{sec}$ as a function of time is shown in Figure 4.30. Even though the overall shape of the beat behavior is similar to that of the corresponding CLPT case (Figure 4.18), the amplitudes of alternate peaks are much lower. The corresponding plot of the shear deflection $w_{s}$ is presented as Figure 4.31. The shape is similar to that of $w_{b}$ but one order lower in magnitude.

The $w_{b}$ and $w_{s}$ plots for the same laminate when $\Omega=20 \mathrm{rad} / \mathrm{sec}$ are seen in Figures 4.32 and 4.33 . The number of beats is double that of the case of $\Omega=10 \mathrm{rad} / \mathrm{sec}$ due to the doubling of $\Omega$. The total deflection $w=\left(w_{b}+w_{s}\right)$ for this case is shown in Figure 4.34. The axial deflection, $u$, at the right end is plotted in Figure 4.35 and, as was 


\begin{tabular}{|c|c|c|c|c|c|c|c|}
\hline Lay-up & $\begin{array}{c}\Omega \\
(\mathrm{rad} / \mathrm{sec})\end{array}$ & $\begin{array}{c}w_{b} \\
(\mathrm{~m}) \\
t=0\end{array}$ & $\begin{array}{c}w_{s} \\
(\mathrm{~m}) \\
t=0\end{array}$ & $\begin{array}{c}w_{b} \\
(\mathrm{~m}) \\
\max \end{array}$ & $\begin{array}{c}w_{s} \\
(\mathrm{~m}) \\
\max \end{array}$ & $\begin{array}{c}w \\
(\mathrm{~m}) \\
\left(w_{b}+w_{s}\right)\end{array}$ & $\begin{array}{c}\text { Magnification } \\
\frac{\text { Factor }}{(w)_{\max }}\end{array}$ \\
\hline $0 / 45 /-45$ & 10 & 0.01 & 0.00034075 & 0.01385 & 0.0004705 & 0.014320 & 1.3848 \\
\hline $0 / 45 /-45$ & 20 & 0.01 & 0.00034075 & 0.01397 & 0.0004967 & 0.014460 & 1.3984 \\
\hline $0 / 20 /-20$ & 10 & 0.01 & 0.00093484 & 0.01439 & 0.0012970 & 0.015687 & 1.4346 \\
\hline $0 / 20 /-20$ & 20 & 0.01 & 0.00093484 & 0.01436 & 0.0013320 & 0.015692 & 1.4350 \\
\hline$[0 / 45 /-45]_{s}$ & 20 & 0.01 & 0.00111010 & 0.01418 & 0.0015600 & 0.015740 & 1.4167 \\
\hline
\end{tabular}

Table 4.7 Maximum tip deflections of beams based on FSDT

seen for CLPT, the magnitude is one order lower than $w$. The midplane shear strain $\gamma$ at the left end, presented in Figure 4.36, is two orders lower than that of $w$. Figure 4.37 presents the slope $w_{b}^{\prime}$ at the left end of the beam and this also exhibits a beat-like phenomenon. The quantity $w_{b}^{y}$, which represents the twist angle is plotted in Figure 4.38 and it should be noted that this is one order lower than that of the slope $w_{b}^{\prime}$. The response of the laminate $[0 / \pm 20]$ is presented in Figures 4.39 to 4.42 while that of the symmetric laminate $[0 / \pm 45]_{\mathrm{s}}$ in Figures 4.43 and 4.44 . The response frequencies of $[0 / \pm 20]$ and $[0 / \pm 45]_{s}$ are much higher than that of $[0 / \pm 45]$ since the former are much stiffer than the latter.

The magnification factors of the total deflection, for the five cases of FSDT are shown in Table 4.7. The magnification factor ranges from 1.38 to 1.43 and are slightly different from the corresponding CLPT examples. The maximum magnification factors occur in the $[0 / \pm 20]$ laminate. The instantaneous first transverse natural frequency of the 
variable $w_{b}$ for the five examples of FSDT are shown in Figures 4.45 to 4.49. As seen before the axial motion reduces the first natural frequency. The summary of the FSDT results are found in Table 4.8. Compared to the CLPT examples (Tables 4.6), the corresponding FSDT ones exhibit lower instantaneous natural frequencies. The response frequencies $(\omega)$ are also lower than their CLPT counterparts.

\begin{tabular}{|c|c|c|c|c|c|c|}
\hline Lay Up & \multicolumn{2}{|c|}{$\Omega$ (forcing - axial) } & $T_{\Omega}$ & \multicolumn{2}{|c|}{$\omega_{1}$ (natural) } & \multirow{2}{*}{$\begin{array}{c}\omega \text { (response) } \\
\mathrm{Hz}\end{array}$} \\
\cline { 2 - 6 } & $\mathrm{rad} / \mathrm{sec}$ & $\mathrm{Hz}$ & $\mathrm{sec}$ & $\mathrm{rad} / \mathrm{sec}$ & $\mathrm{Hz}$ & \\
\hline $0 / 45 /-45$ & 10 & 1.59 & 0.628 & $1176-1341$ & $187.17-213.43$ & 198 \\
\hline $0 / 45 /-45$ & 20 & 3.18 & 0.314 & $1176-1341$ & $187.17-213.43$ & 198 \\
\hline $0 / 20 /-20$ & 10 & 1.59 & 0.628 & $1902-2196$ & $302.71-349.50$ & 324 \\
\hline $0 / 20 /-20$ & 20 & 3.18 & 0.314 & $1902-2196$ & $302.71-349.50$ & 324 \\
\hline$[0 / 45 /-45]_{\mathrm{s}}$ & 20 & 3.18 & 0.314 & $2032-2309$ & $323.40-367.49$ & 345 \\
\hline
\end{tabular}

Table 4.8 Instantaneous first natural frequency of beams based on FSDT

\subsubsection{Moving Beam Simulation using HSDT}

The main objective of this thesis is to simulate the moving beam using HSDT. In this section the response of composite moving beams based on HSDT is presented. To compare the results with CLPT and FSDT, beams with same properties and lay-up configuration as that of the CLPT and FSDT are used. The computer program for this case is run for a time interval of 0 to $1 \mathrm{sec}$ for one of the five cases. For the other cases, the program is run for a period of $0.5 \mathrm{sec}$ since the finite element based on HSDT has more number of degrees of freedom (34) compared to CLPT (22 d.o.f) and FSDT (29 d.o.f) and demands considerable run time. 


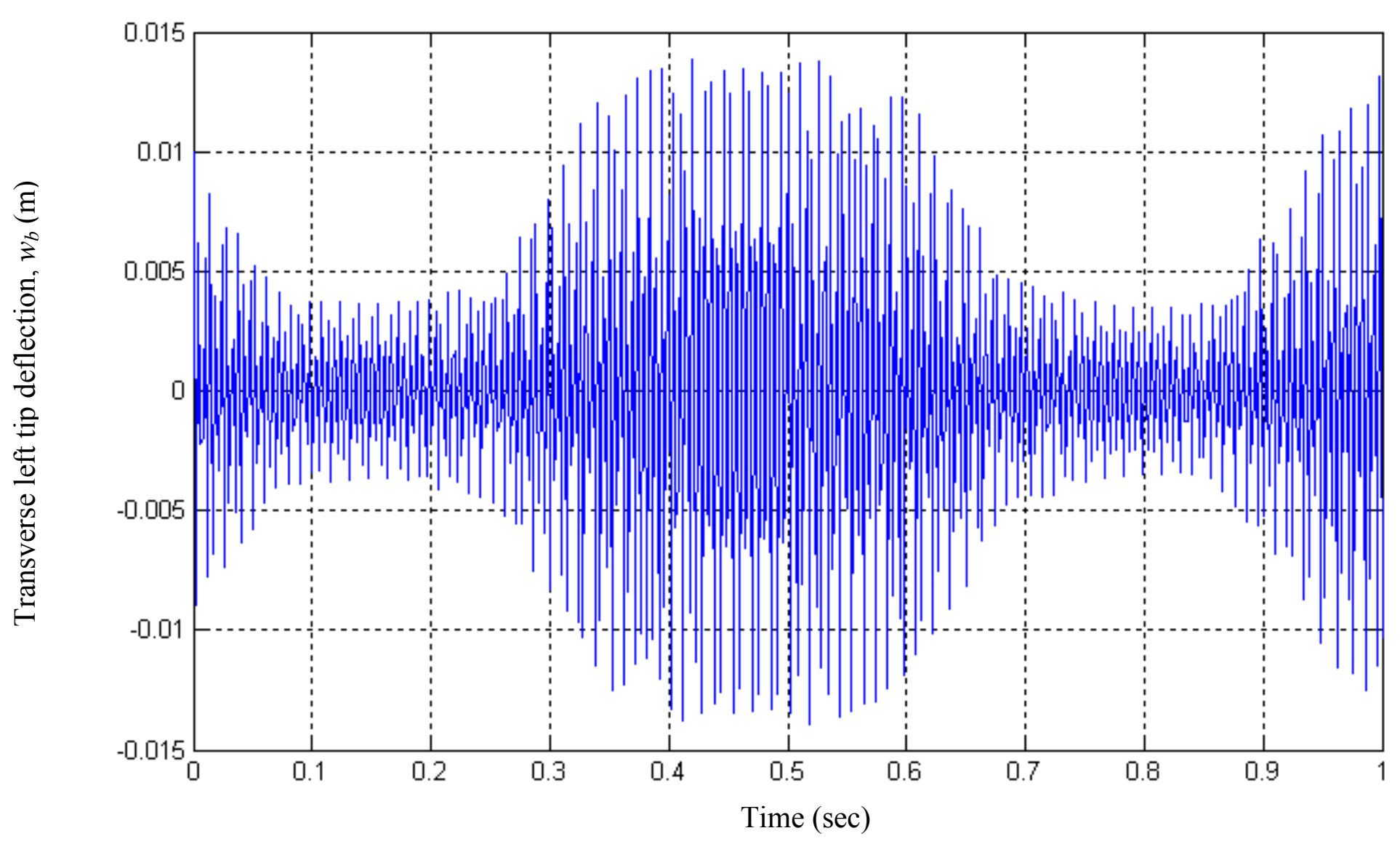

Figure 4.30 Transverse left tip deflection $w_{b}$, FSDT, $\Omega=10 \mathrm{rad} / \mathrm{sec}$ 
Lay-up $=0 / 45 /-45$

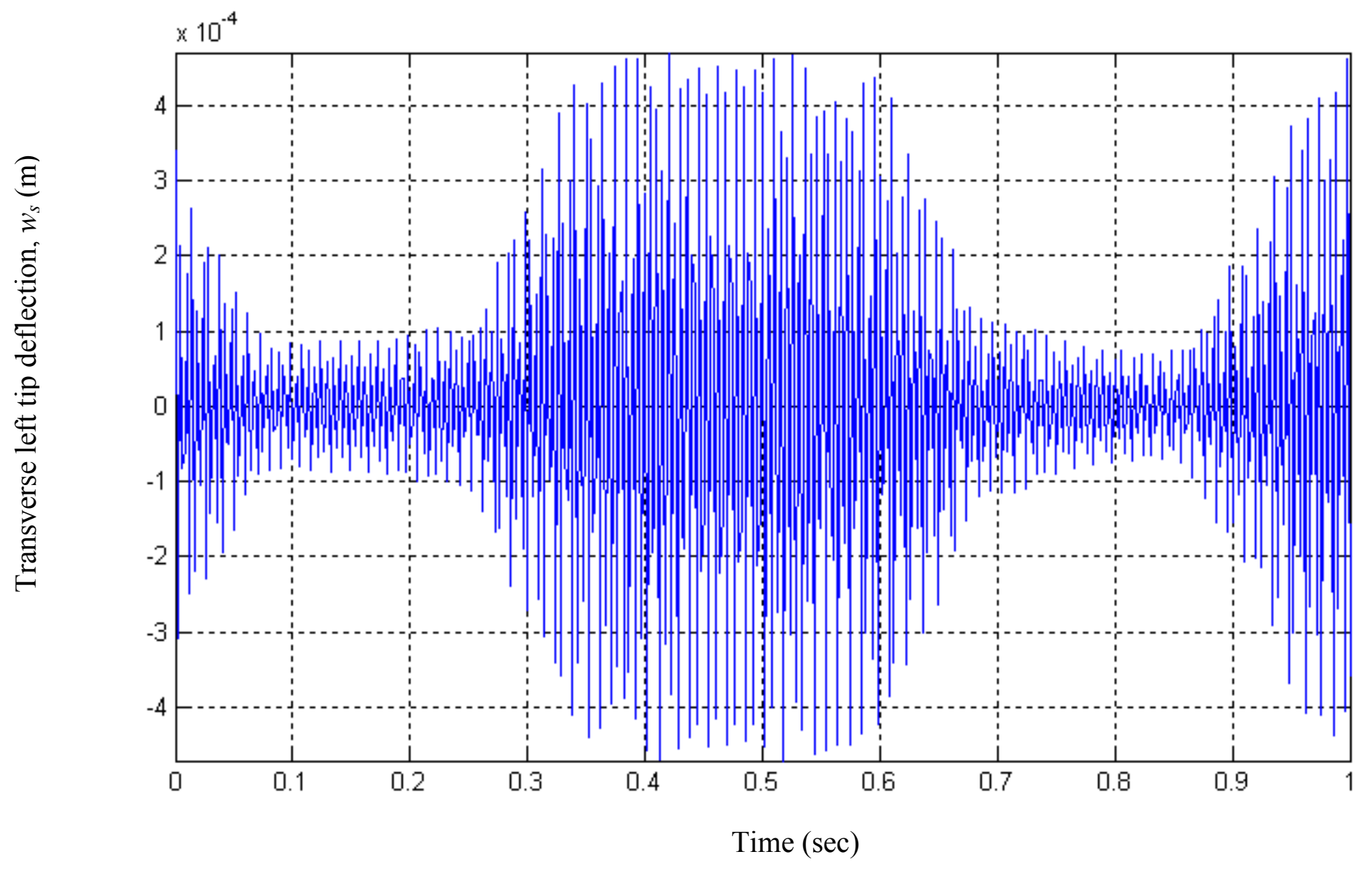

Figure 4.31 Transverse left tip deflection $w_{s}$, FSDT, $\Omega=10 \mathrm{rad} / \mathrm{sec}$ 
Lay-up $=0 / 45 /-45$

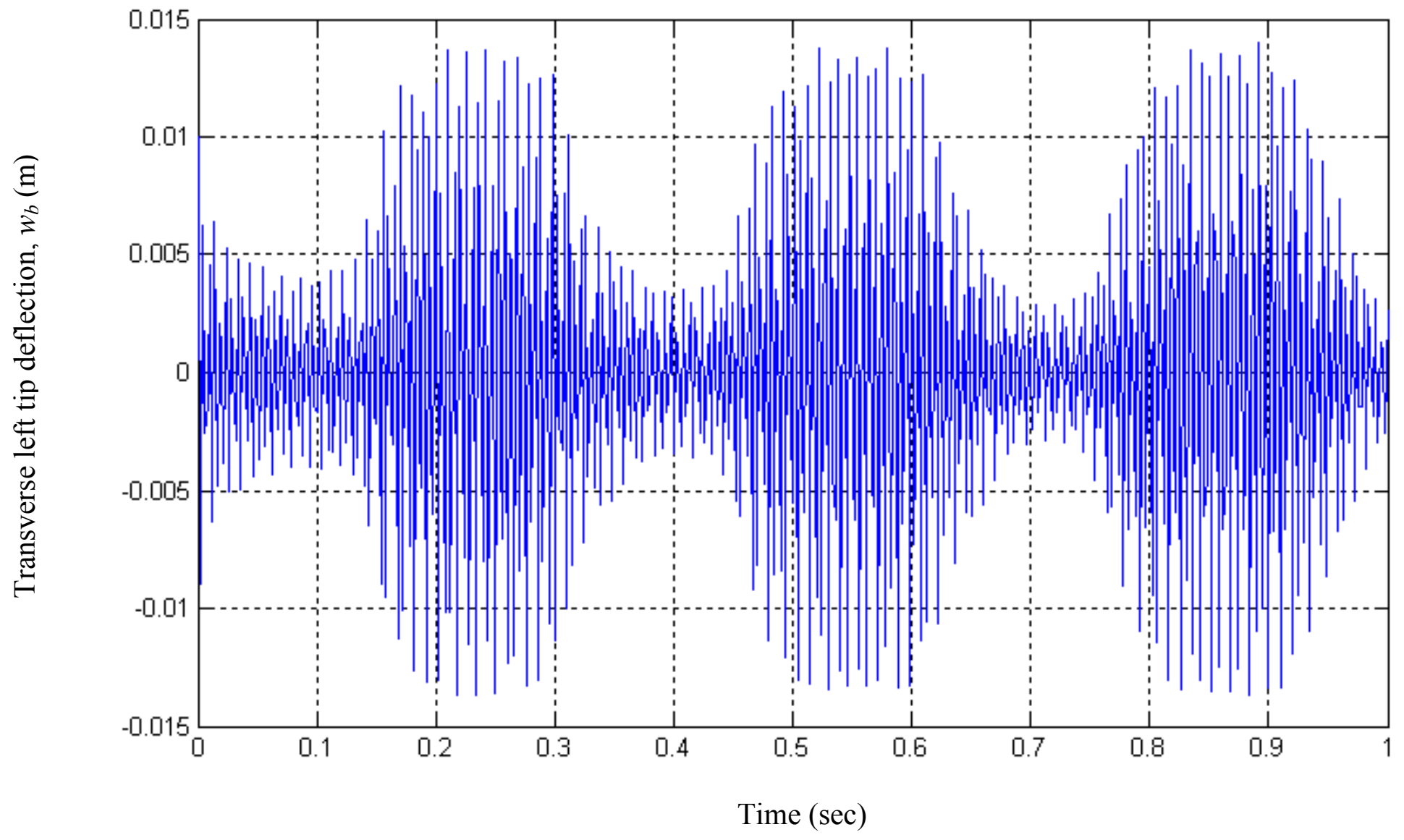

Figure 4.32 Transverse left tip deflection $w_{b}$, FSDT, $\Omega=20 \mathrm{rad} / \mathrm{sec}$ 
Lay-up $=0 / 45 /-45$

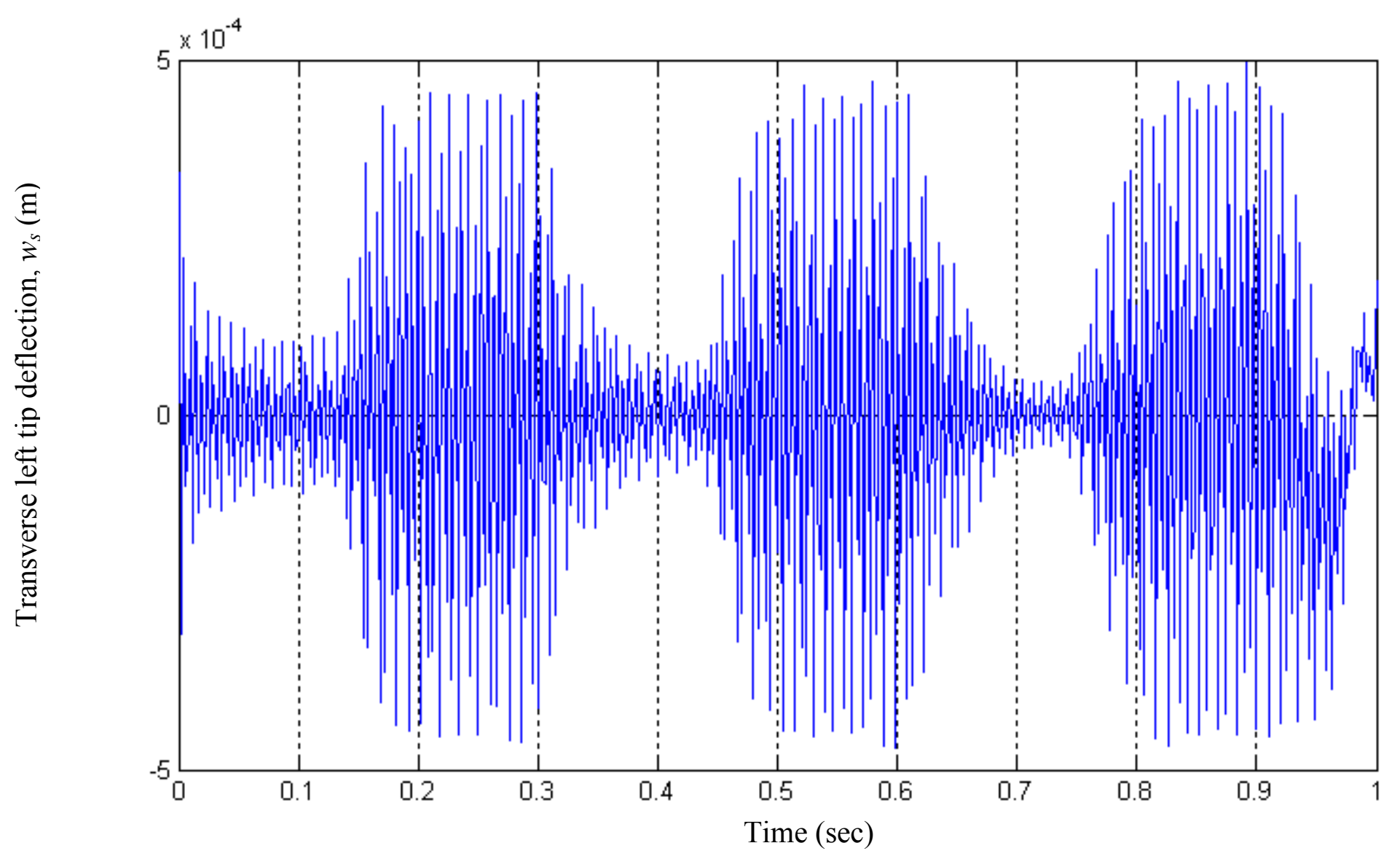

Figure 4.33 Transverse left tip deflection $w_{s}$, FSDT, $\Omega=20 \mathrm{rad} / \mathrm{sec}$ 
Lay-up $=0 / 45 /-45$

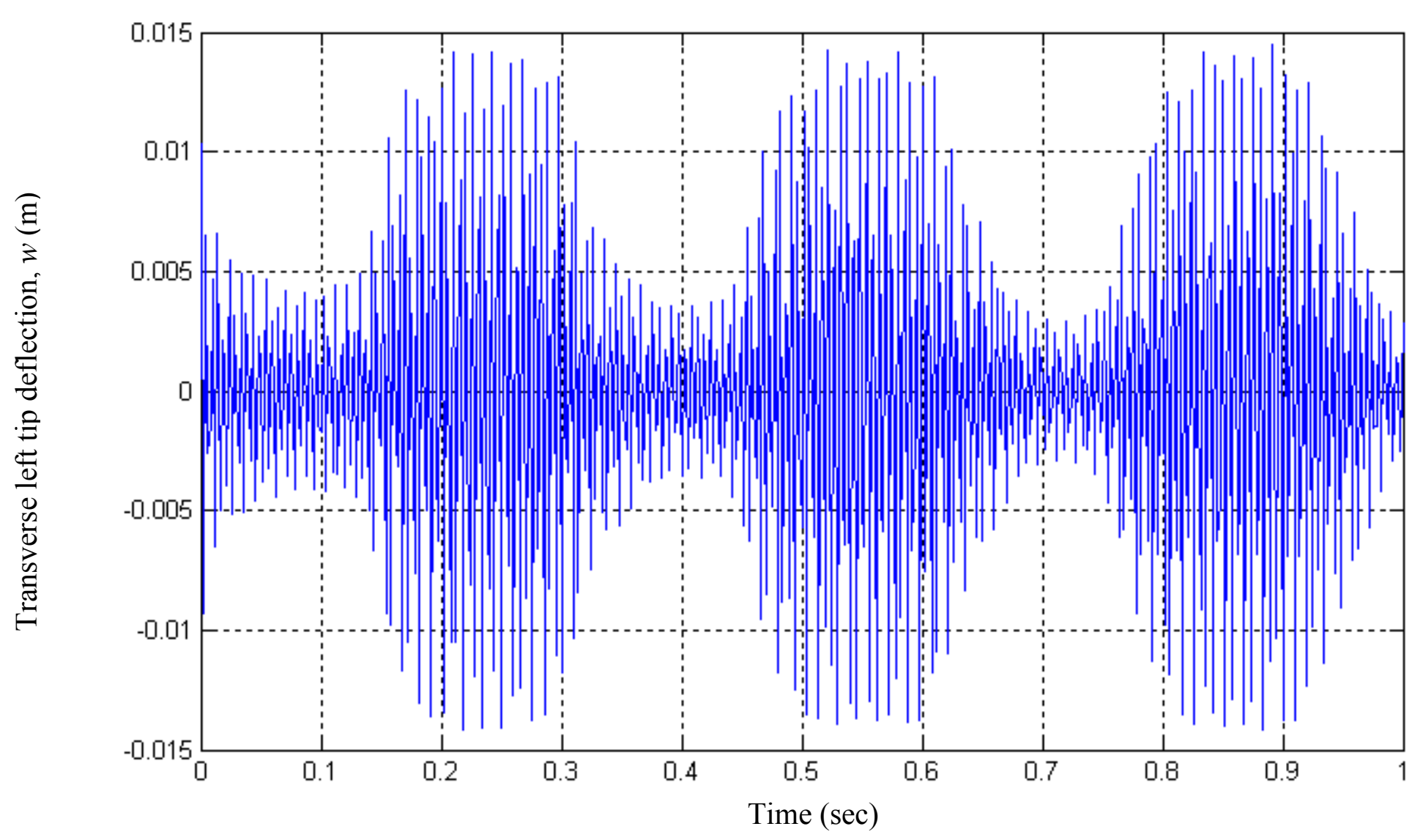

Figure 4.34 Transverse left tip deflection $w$, FSDT, $\Omega=20 \mathrm{rad} / \mathrm{sec}$ 


$$
\text { Lay-up }=0 / 45 /-45
$$

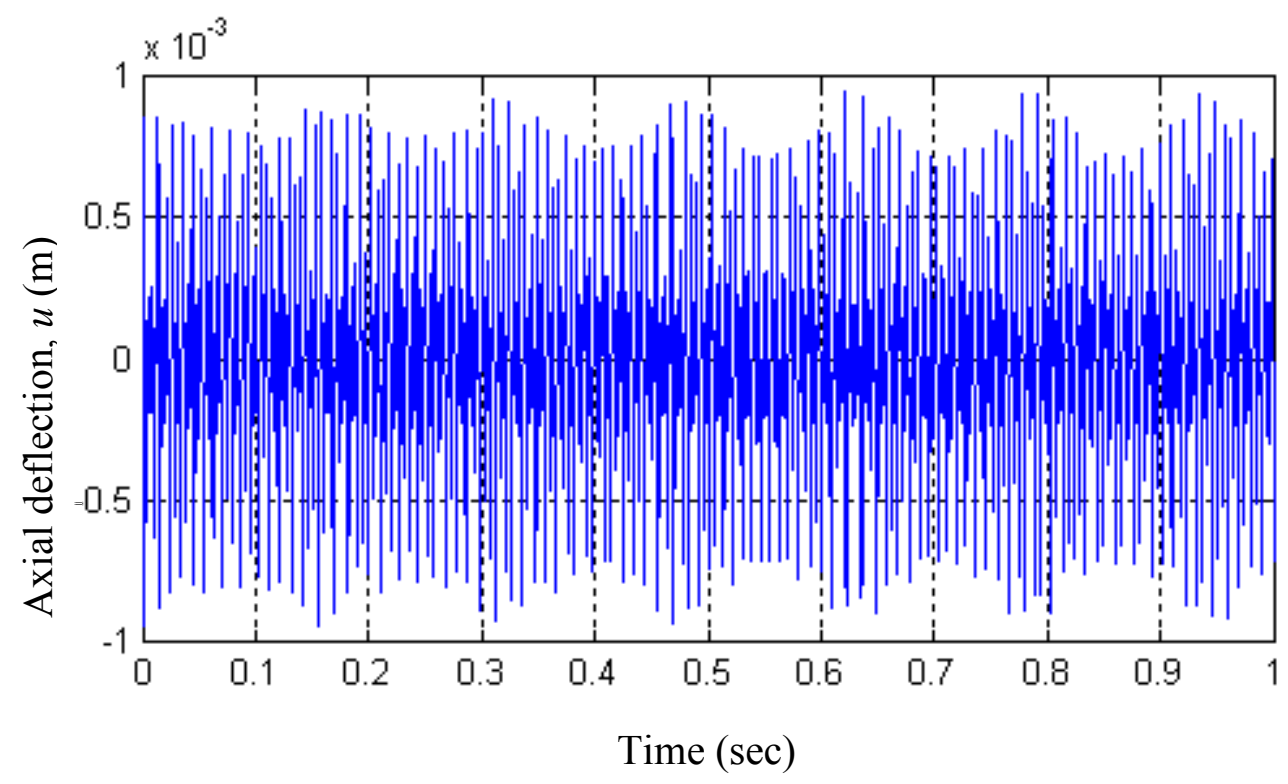

Figure 4.35 Axial right tip deflection $u$, FSDT, $\Omega=20 \mathrm{rad} / \mathrm{sec}$

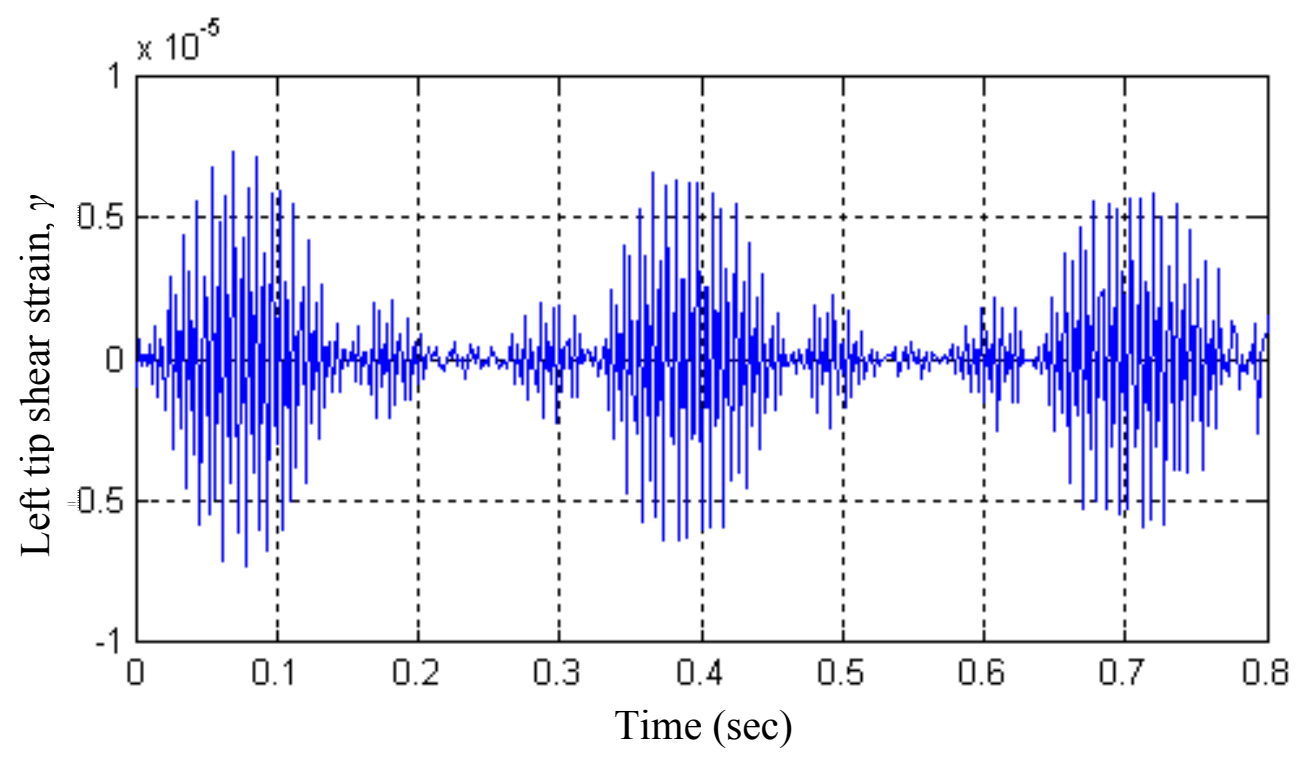

Figure 4.36 Left tip shear strain $\gamma$, FSDT, $\Omega=20 \mathrm{rad} / \mathrm{sec}$ 
Lay-up $=0 / 45 /-45$

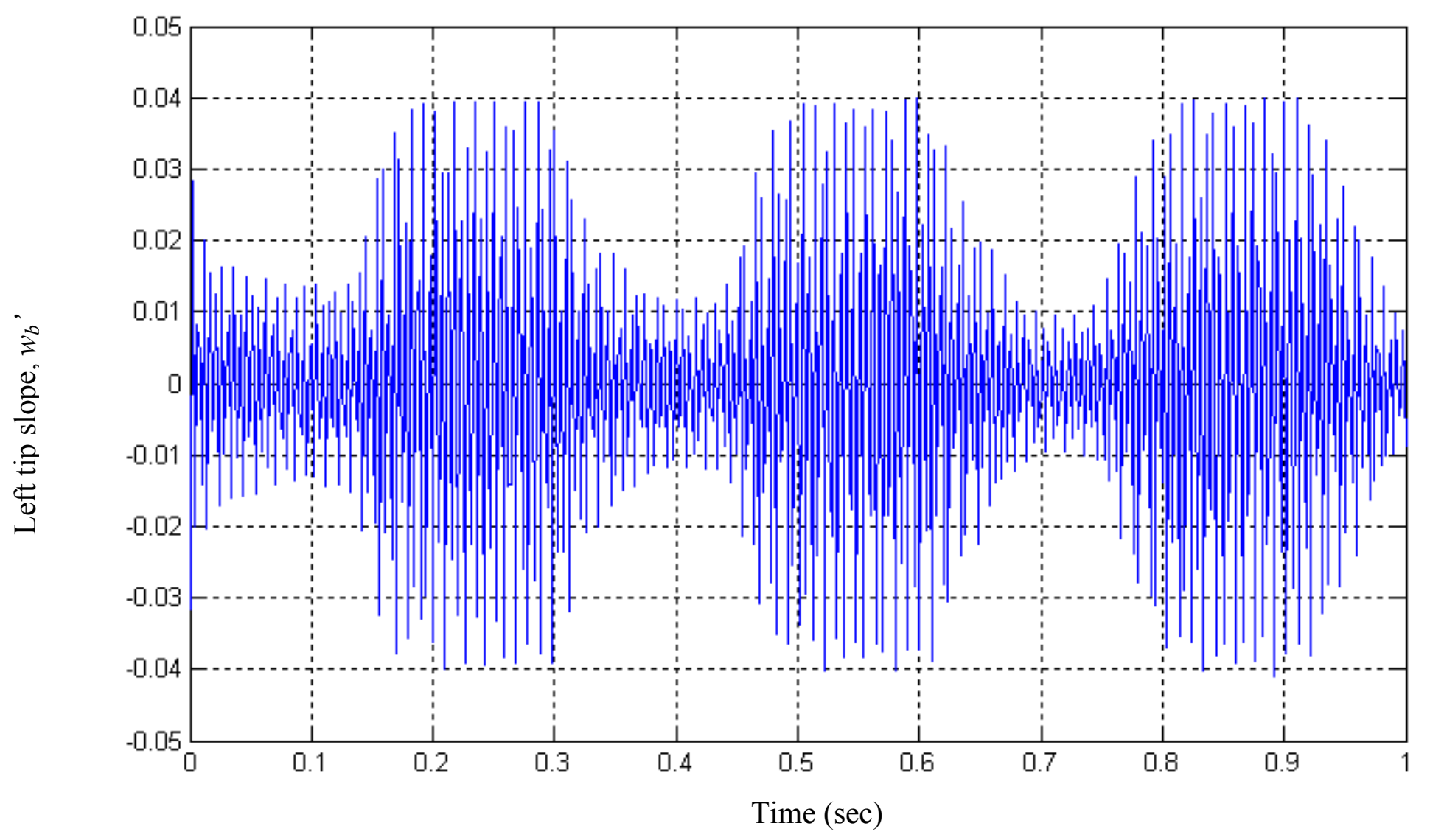

Figure 4.37 Left tip slope $w_{b}{ }^{\prime}$, FSDT, $\Omega=20 \mathrm{rad} / \mathrm{sec}$ 


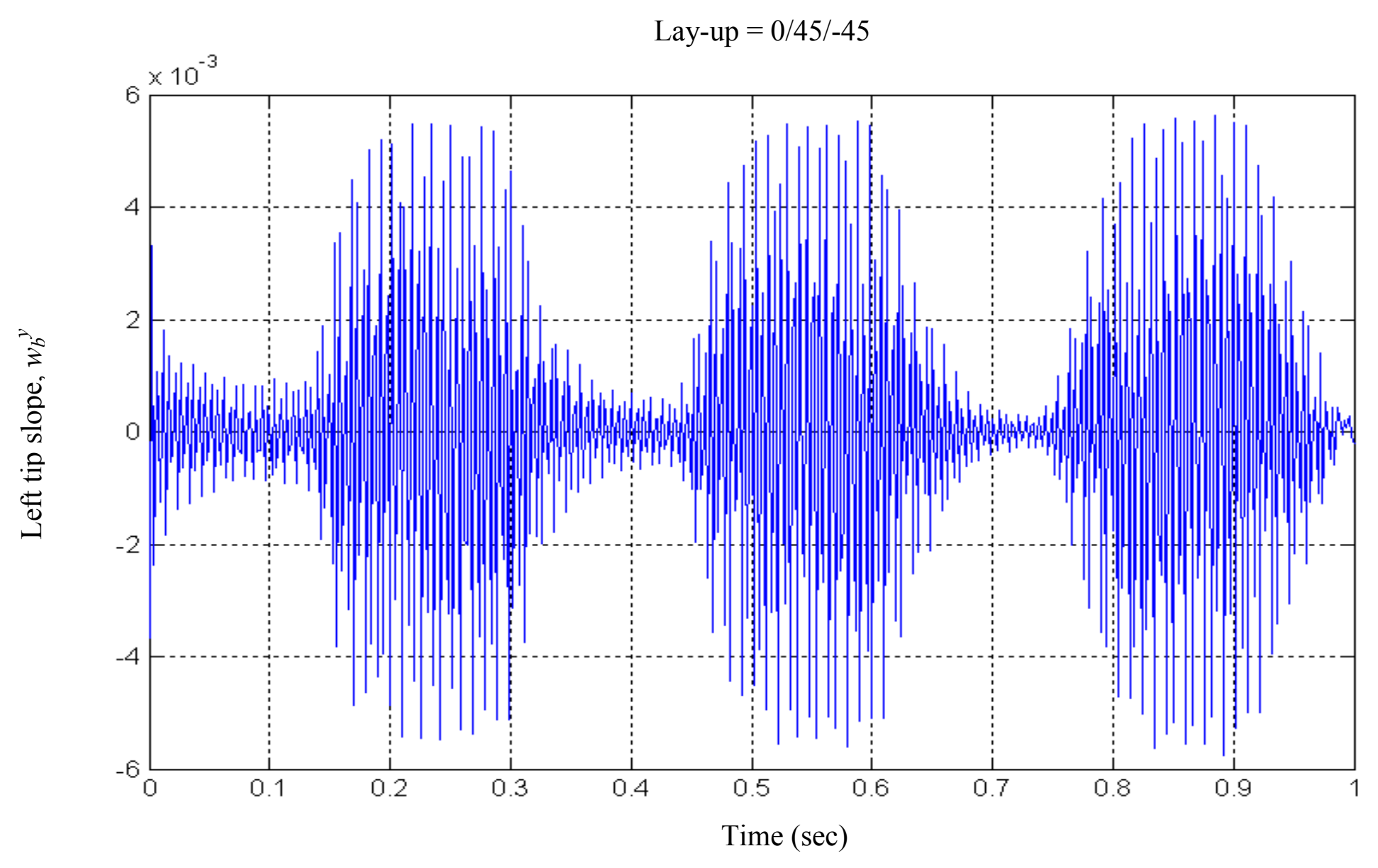

Figure 4.38 Left tip slope $w_{b}{ }^{y}$, FSDT, $\Omega=20 \mathrm{rad} / \mathrm{sec}$ 
Lay-up $=0 / 20 /-20$

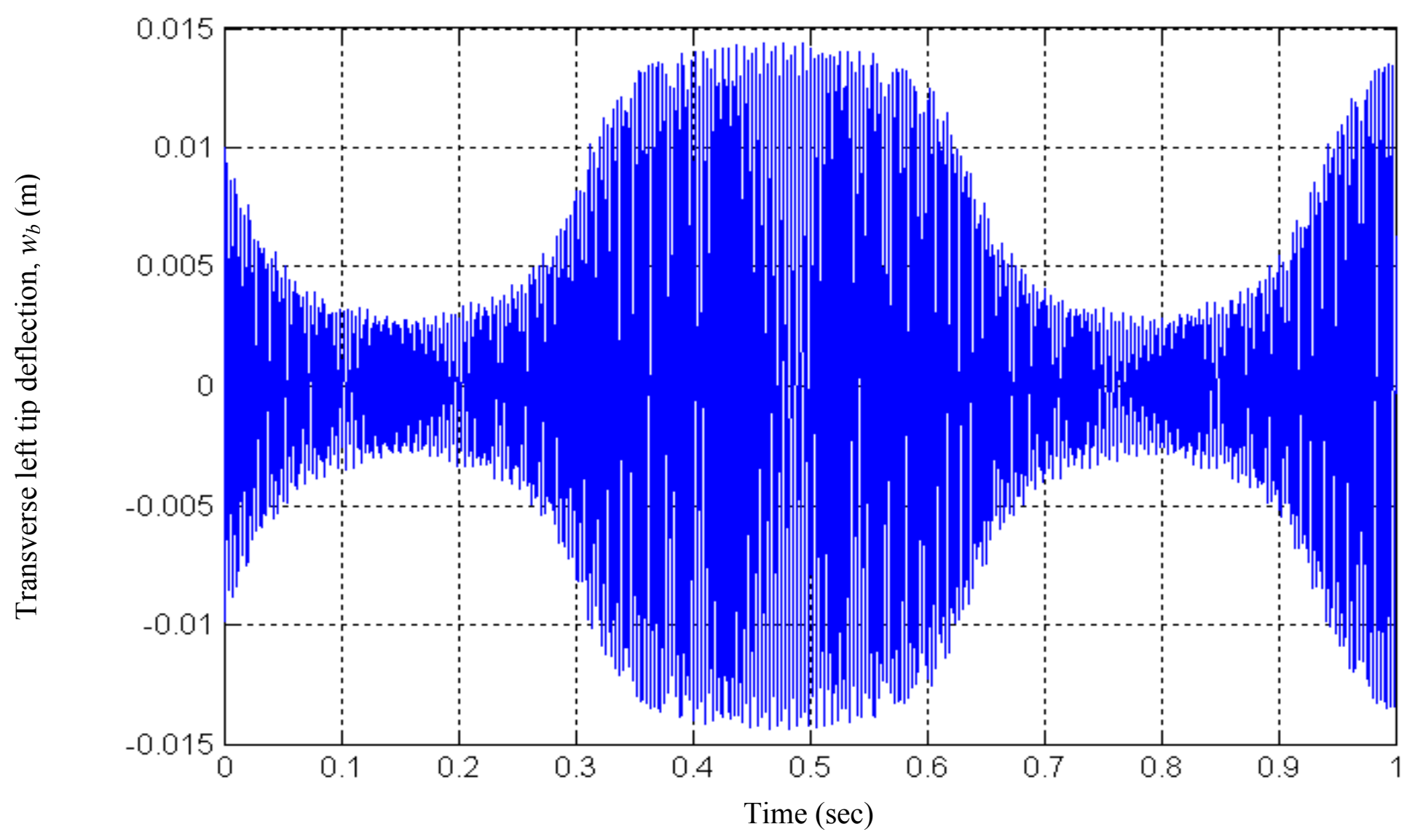

Figure 4.39 Transverse left tip deflection $w_{b}$, FSDT, $\Omega=10 \mathrm{rad} / \mathrm{sec}$ 
Lay-up $=0 / 20 /-20$

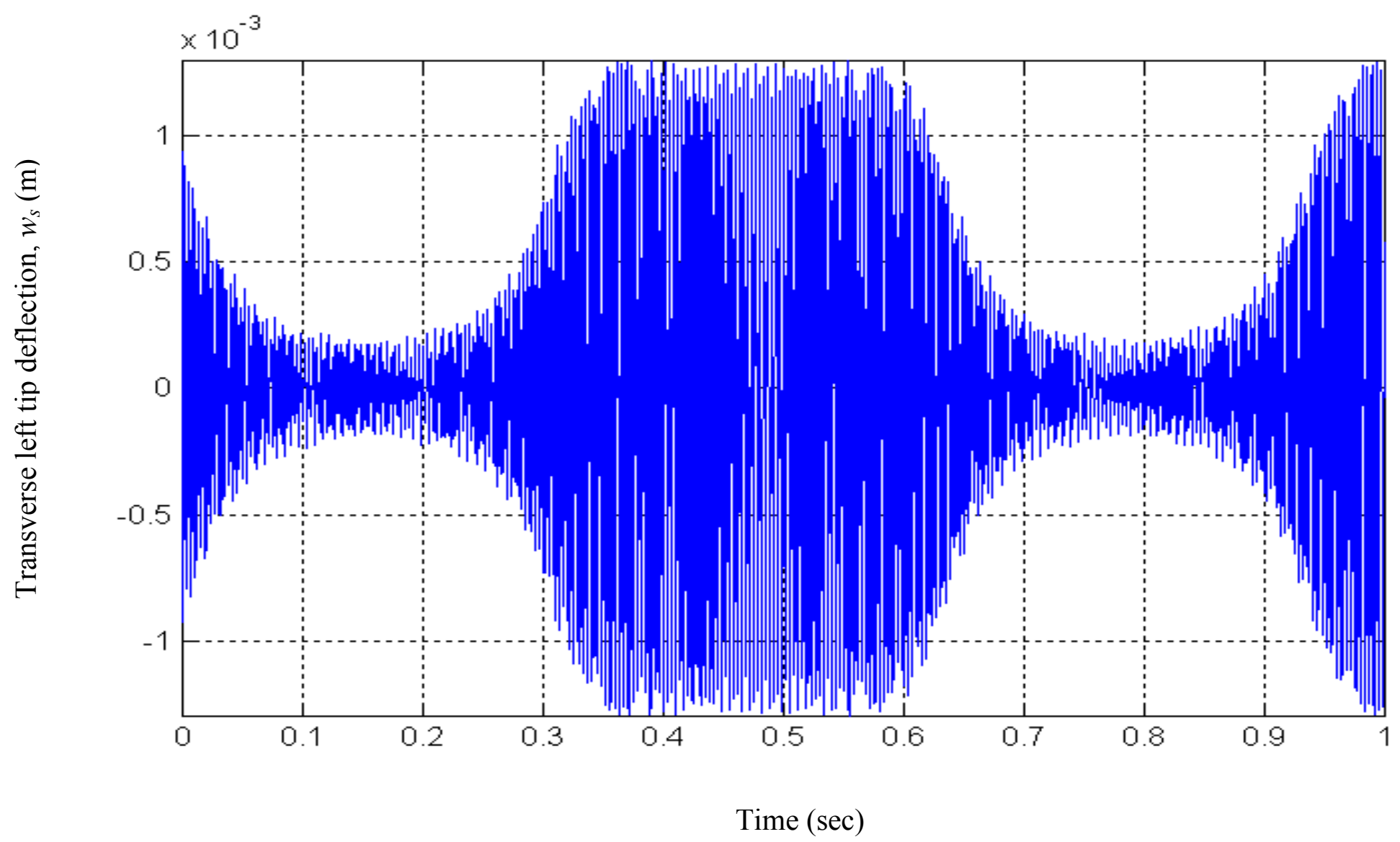

Figure 4.40 Transverse left tip deflection $w_{s}$, FSDT, $\Omega=10 \mathrm{rad} / \mathrm{sec}$ 
Lay-up $=0 / 20 /-20$

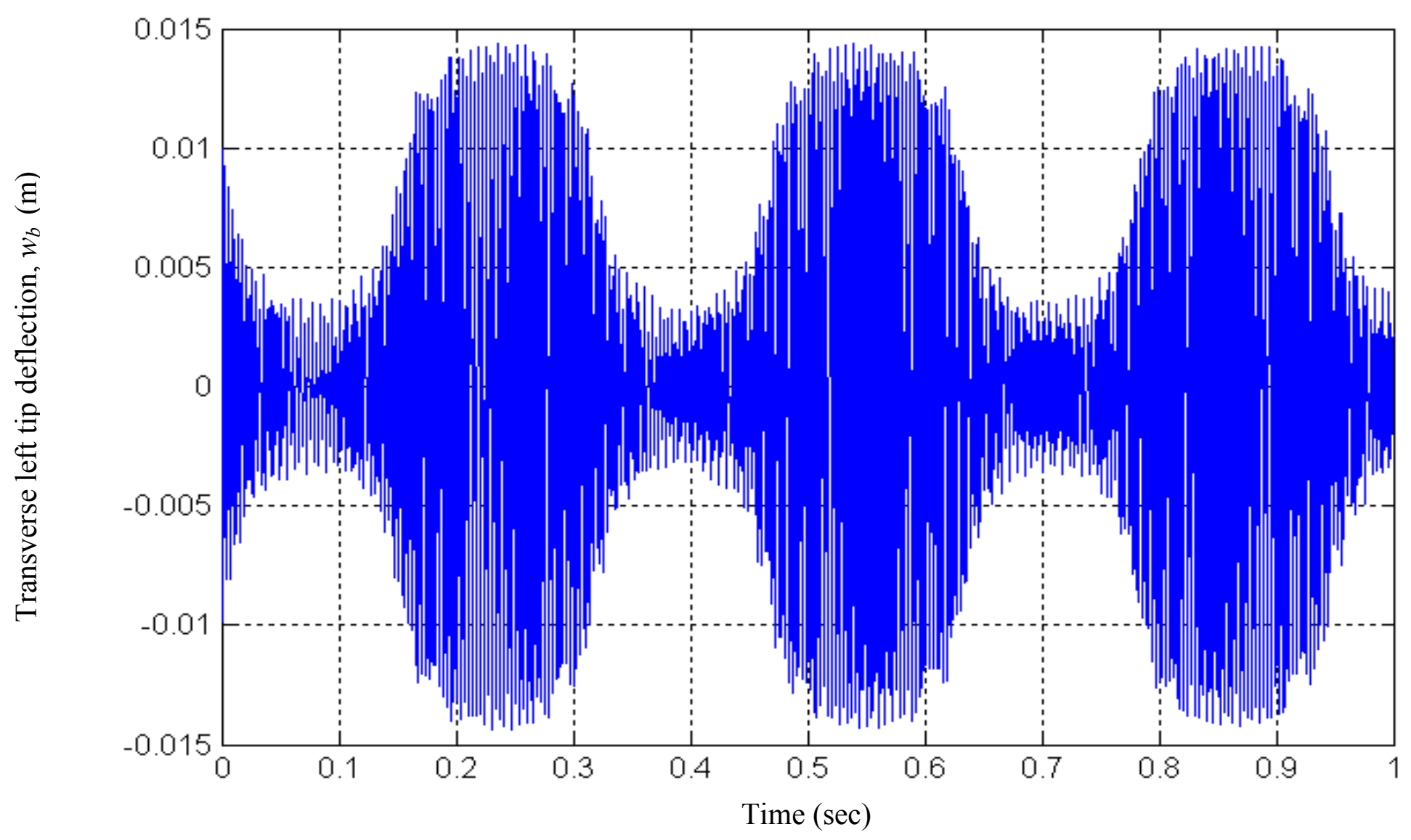

Figure 4.41 Transverse left tip deflection $w_{b}$, FSDT, $\Omega=20 \mathrm{rad} / \mathrm{sec}$ 
Lay-up $=0 / 20 /-20$

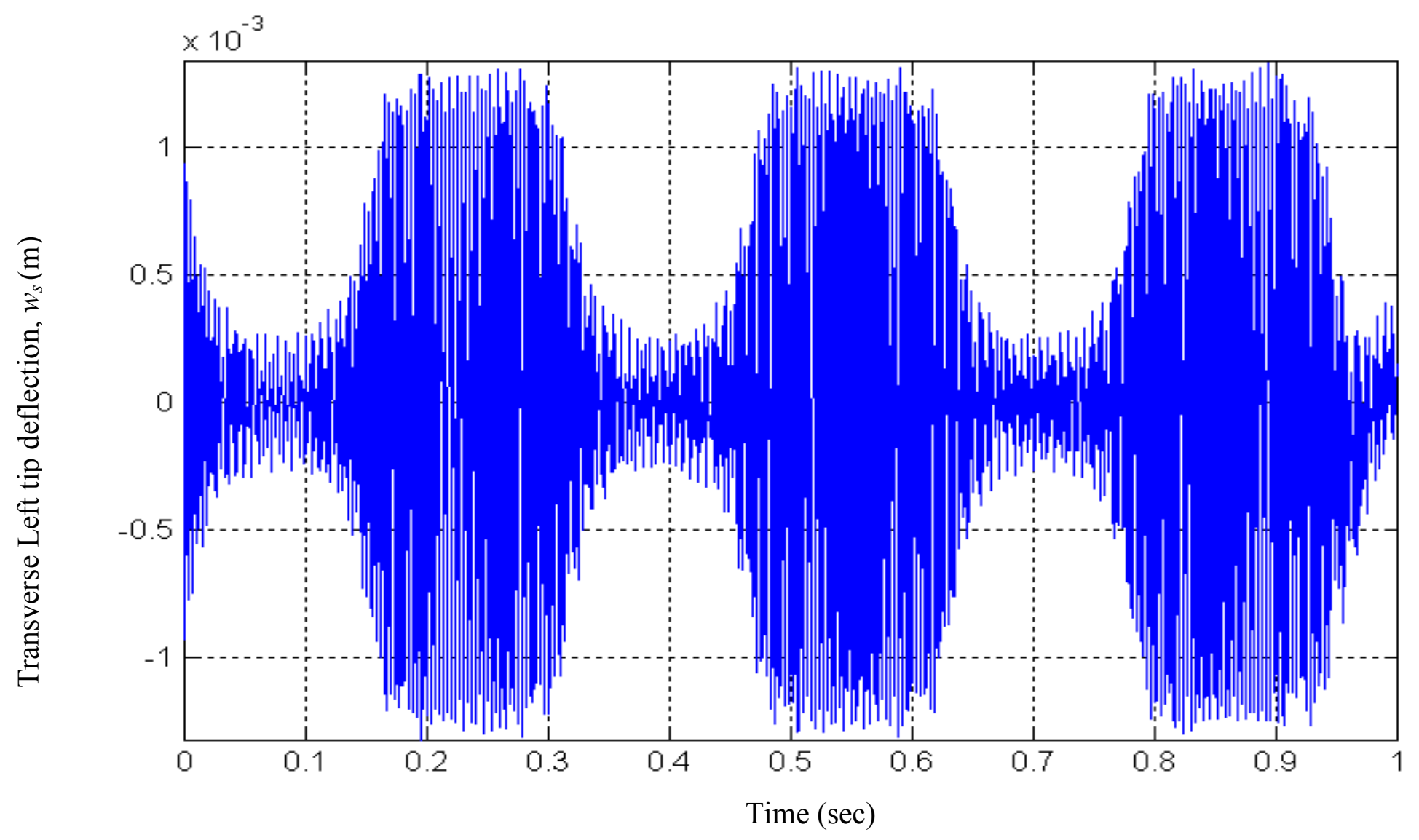

Figure 4.42 Transverse Left tip deflection, $w_{s}$, FSDT, $\Omega=20 \mathrm{rad} / \mathrm{sec}$ 
Lay-up $=0 / 45 /-45 /-45 / 45 / 0$

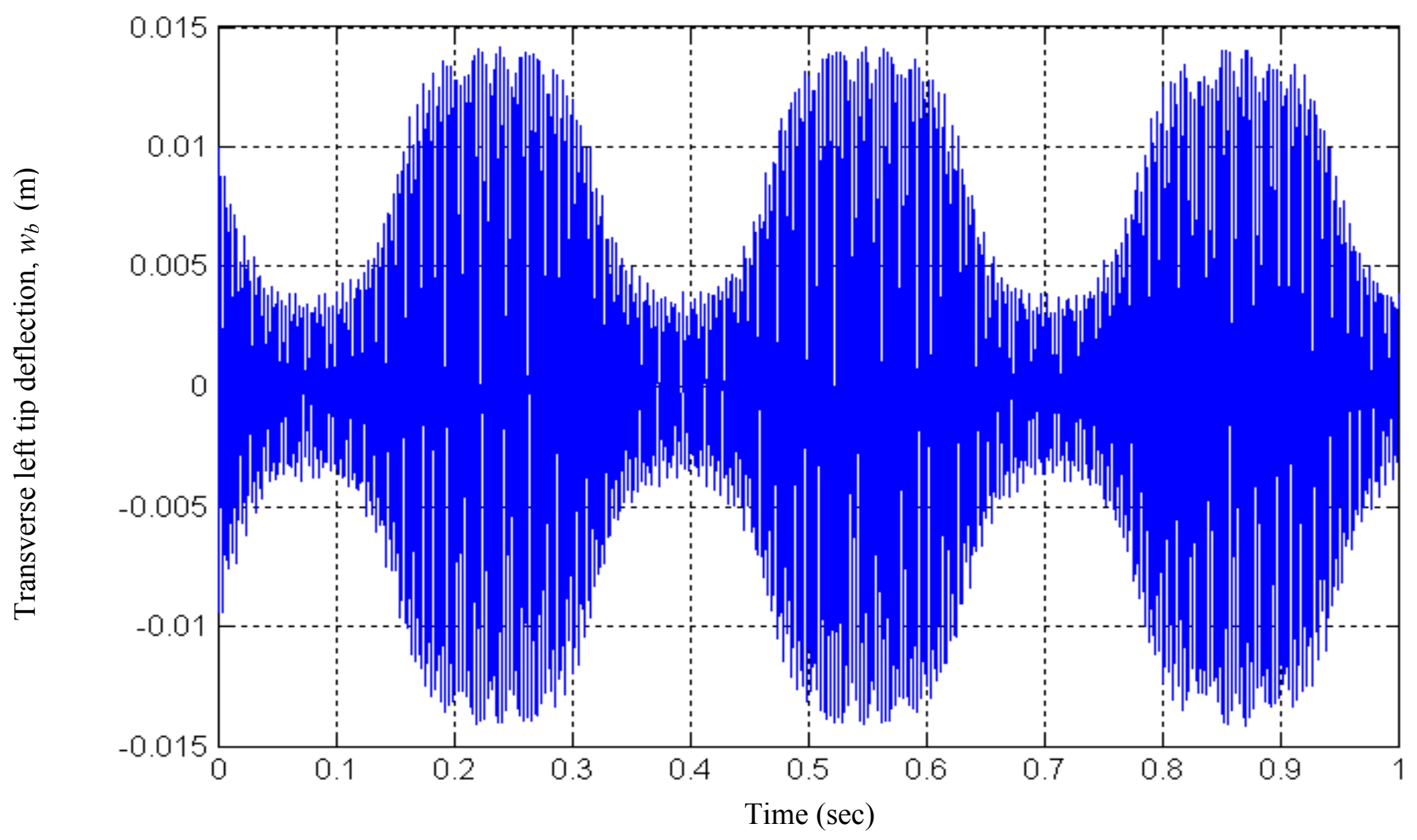

Figure 4.43 Transverse left tip deflection $w_{b}$, FSDT, $\Omega=20 \mathrm{rad} / \mathrm{sec}$ 
Lay-up $=0 / 45 /-45 /-45 / 45 / 0$

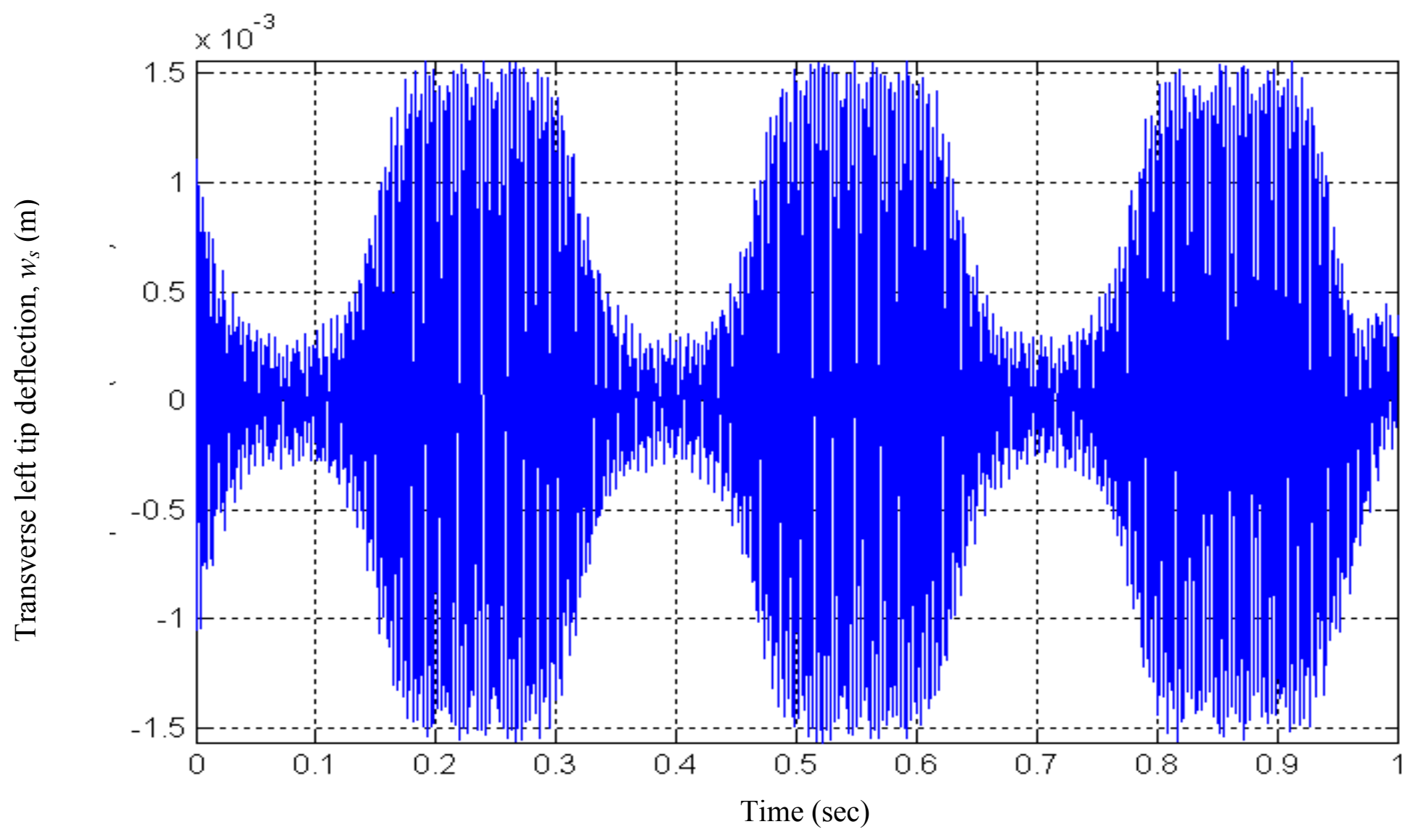

Figure 4.44 Transverse left tip deflection, $w_{s}$, FSDT, $\Omega=20 \mathrm{rad} / \mathrm{sec}$ 


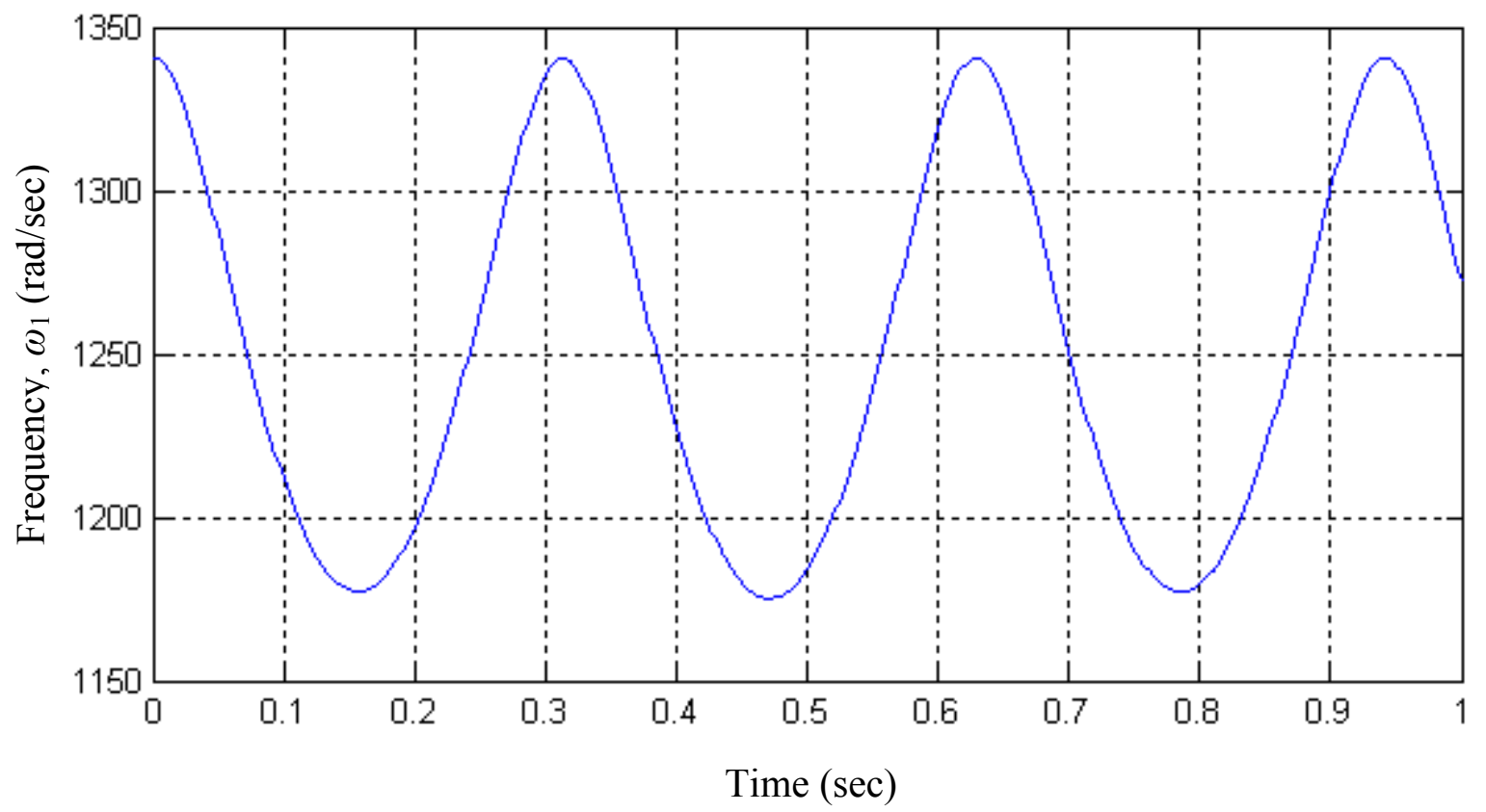

Figure 4.45 First transverse natural frequency $\omega_{1}$, of $[0 / \underline{45}]$, FSDT, $\Omega=10 \mathrm{rad} / \mathrm{sec}$

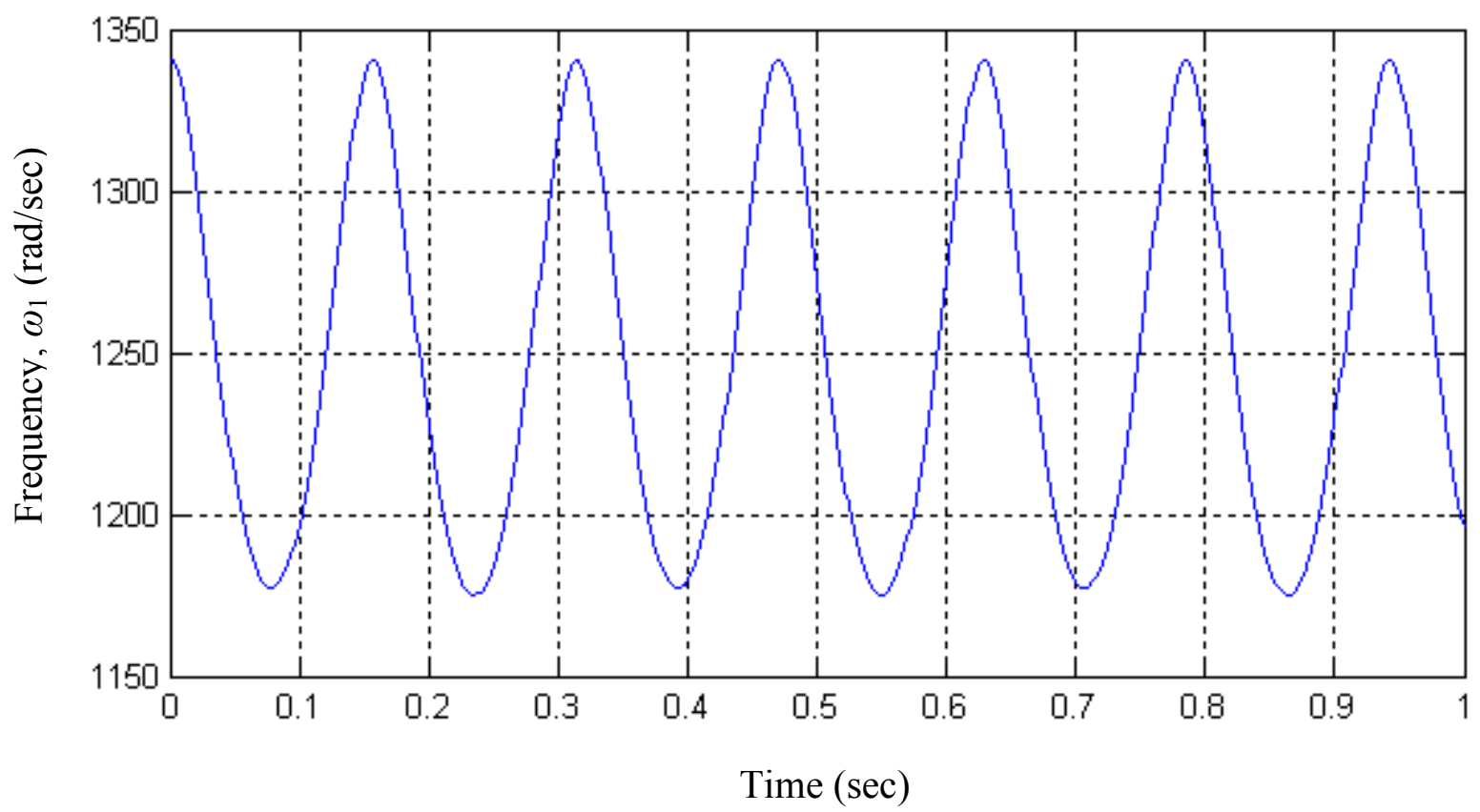

Figure 4.46 First transverse natural frequency $\omega_{1}$, of $[0 / \underline{45}]$, FSDT, $\Omega=20 \mathrm{rad} / \mathrm{sec}$ 


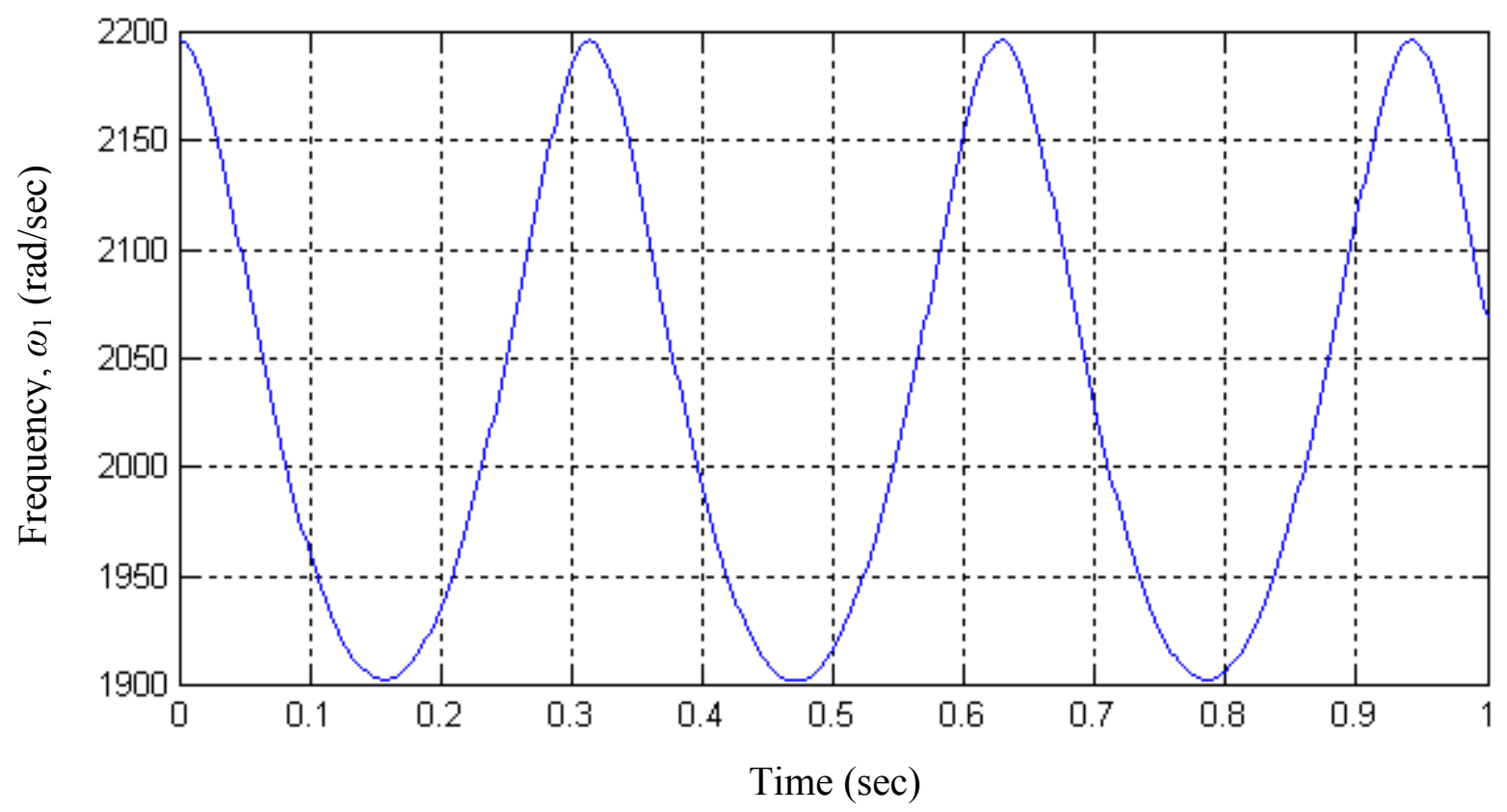

Figure 4.47 First transverse natural frequency $\omega_{1}$, of $[0 / \pm 20], F S D T, \Omega=10 \mathrm{rad} / \mathrm{sec}$

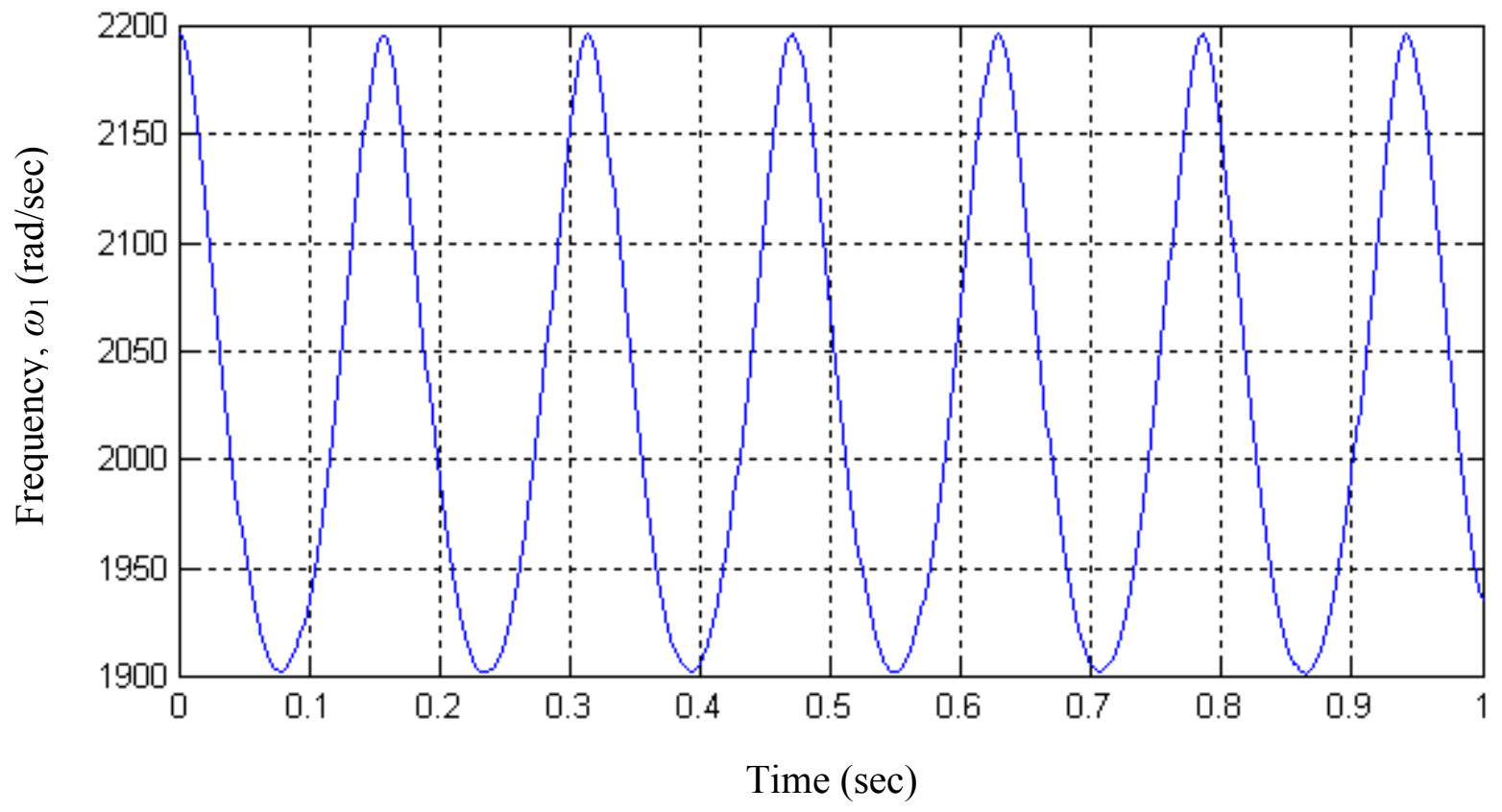

Figure 4.48 First transverse natural frequency $\omega_{1}$, of $[0 / \underline{20}]$, FSDT, $\Omega=20 \mathrm{rad} / \mathrm{sec}$ 


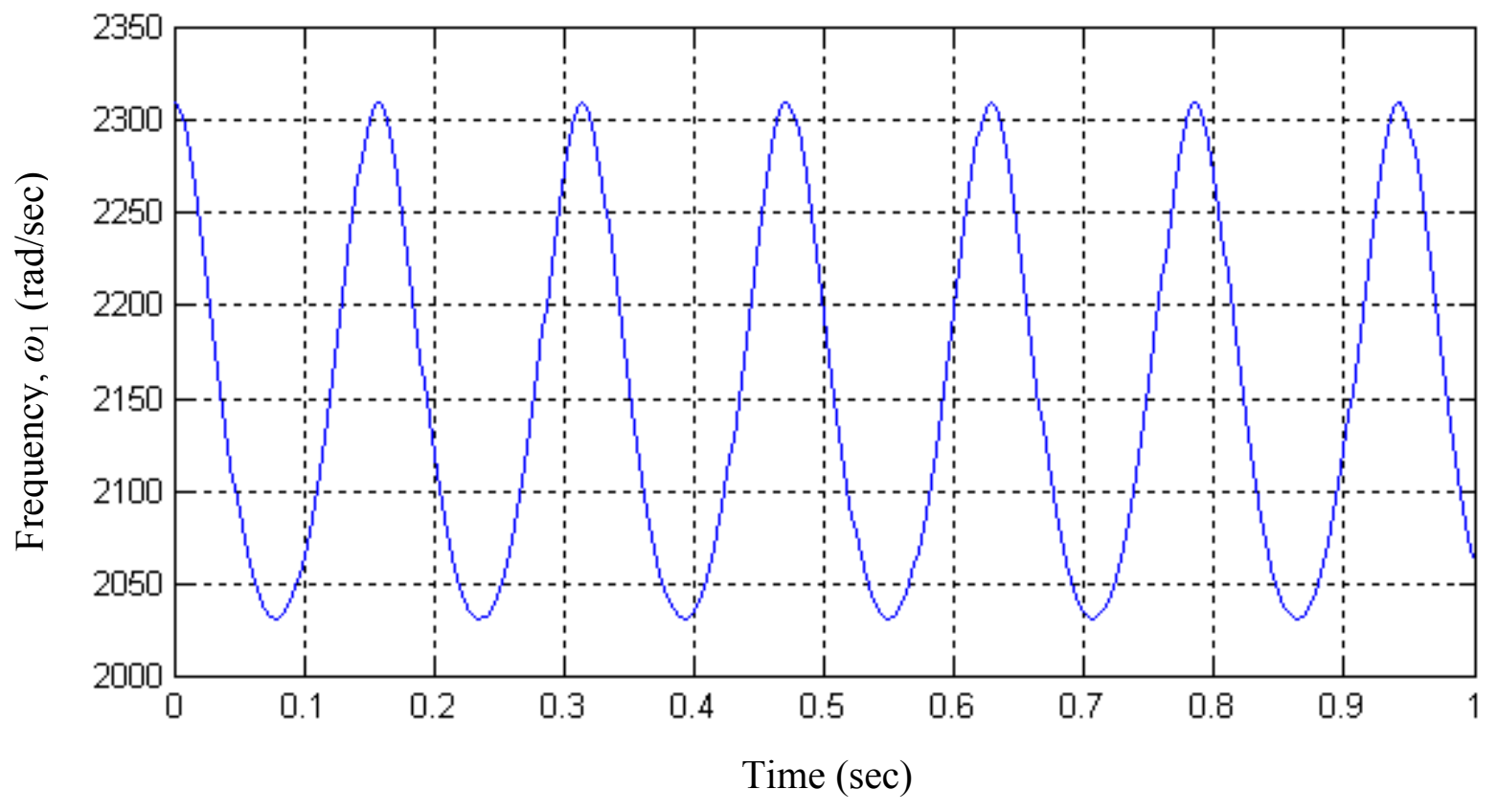

Figure 4.49 First transverse natural frequency $\omega_{1}$, of $[0 / \not 45]_{\mathrm{s}}$ FSDT, $\Omega=10 \mathrm{rad} / \mathrm{sec}$ 
The axial deflection at the left end of the beam is fixed to avoid rigid body motion as in the previous cases. The first mode shape of $w_{b}$ of a symmetrically placed overhang beam is taken as the initial shape. The shape is normalized such that the tip deflections are 0.01 $\mathrm{m}$.

The dependent variables in the HSDT case are $u, w_{b}, w_{s}, \gamma, \mathrm{w}_{\mathrm{b}}^{\mathrm{y}}$, and $w_{s}^{y}$. The results of selected variables are presented for the five run cases. The transverse deflection of the left end of the beam for the laminate $[0 / \pm 45]$ at an axial frequency of $10 \mathrm{rad} / \mathrm{sec}$ over a time interval of 0 to $0.5 \mathrm{sec}$ is shown in Figure 4.50. A beat shape similar to that of the corresponding FSDT (Figure 4.30) case is observed. The corresponding plot of the shear deflection $w_{s}$ is presented as Figure 4.51. The shape is similar to that of $w_{b}$ but one order lower in magnitude. The amplitudes of the bending and the shear components of the transverse left tip deflection are larger than that predicted by FSDT. The shear component based on HSDT is almost twice that of the corresponding value based on FSDT.

The response of the same laminate $[0 / \pm 45]$ at the axial frequency of $20 \mathrm{rad} / \mathrm{sec}$ is seen in Figures 4.52 to 4.58 . The $w_{b}$ and $w_{s}$ plots (Figures 4.52 and 4.53 ) are similar to that of the corresponding FSDT cases. The total deflection $w=\left(w_{b}+w_{s}\right)$ for this case is shown in Figure 4.54. The axial deflection at the right end is shown in Figure 4.55. Similar to the CLPT and FSDT cases, the magnitude is one order lower than $w$. The shear strain $\gamma$, at the left end, is plotted in Figure 4.56. The shear strain, $\gamma$, is of two orders lower 


\begin{tabular}{|c|c|c|c|c|c|c|c|}
\hline Lay-up & $\begin{array}{c}\Omega \\
(\mathrm{rad} / \\
\mathrm{sec})\end{array}$ & $\begin{array}{c}w_{b} \\
(\mathrm{~m}) \\
t=0\end{array}$ & $\begin{array}{c}w_{s} \\
(\mathrm{~m}) \\
t=0\end{array}$ & $\begin{array}{c}w_{b} \\
(\mathrm{~m}) \\
\max \end{array}$ & $\begin{array}{c}w_{s} \\
(\mathrm{~m}) \\
\max \end{array}$ & $\begin{array}{c}w \\
(\mathrm{~m}) \\
\left(w_{b}+w_{s}\right)\end{array}$ & $\begin{array}{c}\text { Magnification } \\
\text { Factor } \\
(w)_{\max }\end{array}$ \\
\hline $0 / 45 /-45$ & 10 & 0.01 & 0.00067642 & 0.01387 & 0.0009187 & 0.0147887 & 1.3852 \\
\hline $0 / 45 /-45$ & 20 & 0.01 & 0.00067642 & 0.01411 & 0.0009522 & 0.0150622 & 1.4108 \\
\hline $0 / 20 /-20$ & 10 & 0.01 & 0.00191940 & 0.01448 & 0.0026470 & 0.0171270 & 1.4369 \\
\hline $0 / 20 /-20$ & 20 & 0.01 & 0.00191940 & 0.01444 & 0.0027450 & 0.0171850 & 1.4418 \\
\hline$[0 / 45 /-45]_{s}$ & 20 & 0.01 & 0.00236010 & 0.01410 & 0.0033250 & 0.0174250 & 1.4098 \\
\hline
\end{tabular}

Table 4.9 Maximum tip deflections of beam based on HSDT

than that of $w$. The slope $w_{b}^{\prime}$ at the left end of the beam is plotted in Figure 4.57 and this exhibits a beat-like phenomenon similar to the FSDT case. The maximum values of $w_{b}^{\prime}$ based on HSDT are slightly higher compared to the values based on FSDT. The quantity $w_{s}^{y}$, which represents the twist angle caused by shear deformation is plotted in Figure 4.58. The response of the laminate $[0 / \pm 20]$ is presented in Figures 4.59 to 4.62 while that of the symmetric laminate $[0 / \pm 45]_{\mathrm{S}}$ in Figs 4.63 and 4.64. In all these cases the transverse shear $w_{s}$ at the left end of the beam is much higher than the corresponding FSDT case.

The magnification factors for the five cases of HSDT are presented in Table 4.9. The magnification factor ranges from 1.38 to 1.43 and is similar to that of the FSDT cases but slightly higher. The instantaneous first transverse natural frequency of the transverse deflection $w_{b}$ for the five examples of HSDT are shown in Figures 4.65 to 4.69. The axial motion reduces the first natural frequency similar to the previous cases. 
The summary of the HSDT results are found in Table 4.10. The HSDT cases exhibit lower instantaneous natural frequencies compared to CLPT and FSDT. The response frequencies of the HSDT cases are also lower than the corresponding FSDT cases.

\begin{tabular}{|c|c|c|c|c|c|c|}
\hline Lay Up & \multicolumn{2}{|c|}{$\Omega$ (forcing - axial) } & $T_{\Omega}$ & \multicolumn{2}{|c|}{$\omega_{1}$ (natural) } & \multirow{2}{*}{$\begin{array}{c}\omega \text { (response) } \\
\text { Hz }\end{array}$} \\
\cline { 2 - 6 } & $\mathrm{rad} / \mathrm{sec}$ & $\mathrm{Hz}$ & $\mathrm{sec}$ & $\mathrm{rad} / \mathrm{sec}$ & $\mathrm{Hz}$ & \\
\hline $0 / 45 /-45$ & 10 & 1.59 & 0.628 & $1158-1322$ & $184.30-210.40$ & 195 \\
\hline $0 / 45 /-45$ & 20 & 3.18 & 0.314 & $1158-1322$ & $184.30-210.40$ & 195 \\
\hline $0 / 20 /-20$ & 10 & 1.59 & 0.628 & $1836-2123$ & $292.21-337.89$ & 312 \\
\hline $0 / 20 /-20$ & 20 & 3.18 & 0.314 & $1836-2123$ & $292.21-337.89$ & 312 \\
\hline$[0 / 45 /-45]_{\mathrm{s}}$ & 20 & 3.18 & 0.314 & $1964-2231$ & $312.58-355.07$ & 334 \\
\hline
\end{tabular}

Table 4.10 Instantaneous first natural frequency of beams based on HSDT 


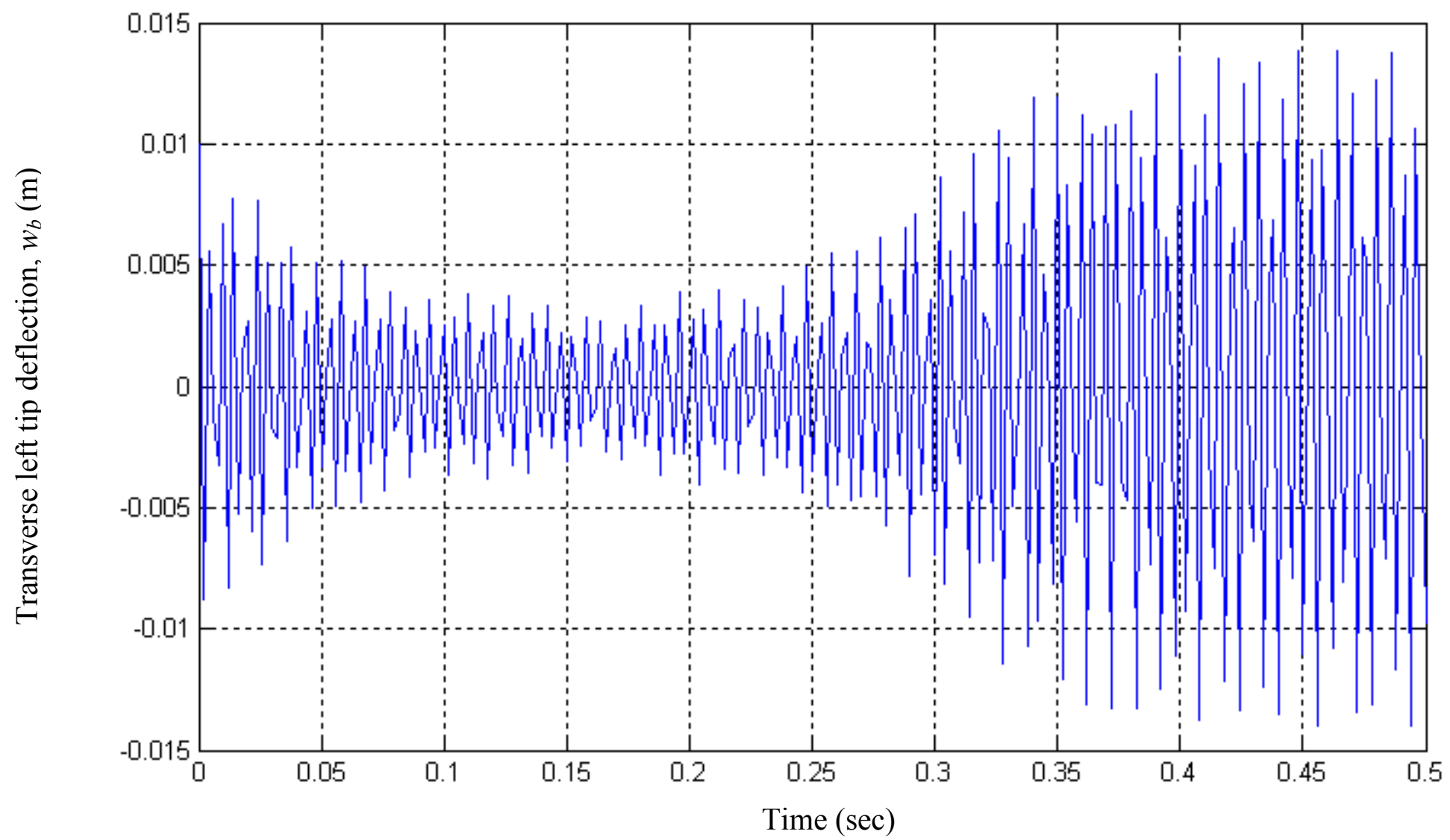

Figure 4.50 Transverse left tip deflection $w_{b}$, HSDT, $\Omega=10 \mathrm{rad} / \mathrm{sec}$ 
Lay-up $=0 / 45 /-45$

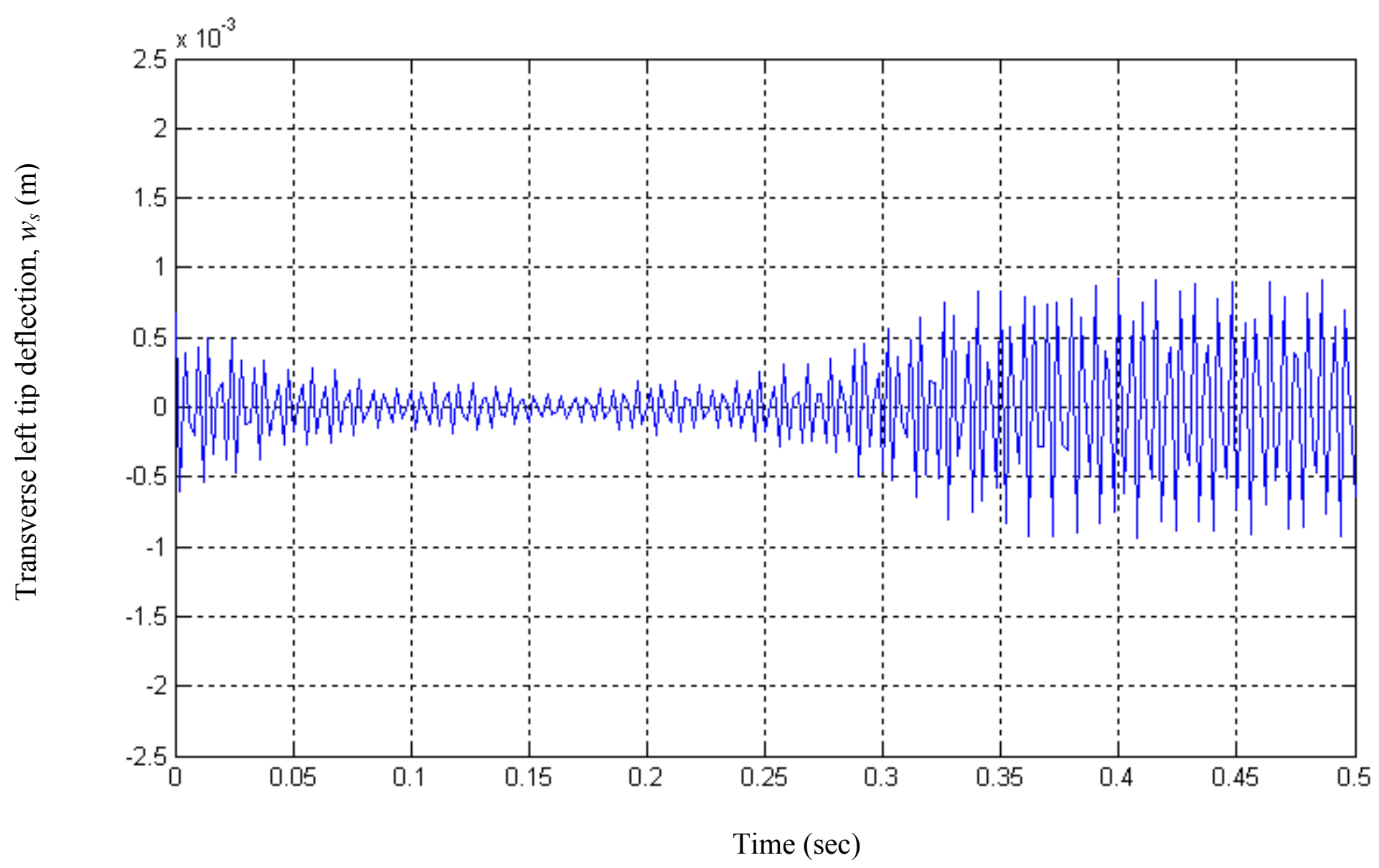

Figure 4.51 Transverse left tip deflection $w_{s}$, HSDT, $\Omega=10 \mathrm{rad} / \mathrm{sec}$ 
Lay-up $=0 / 45 /-45$

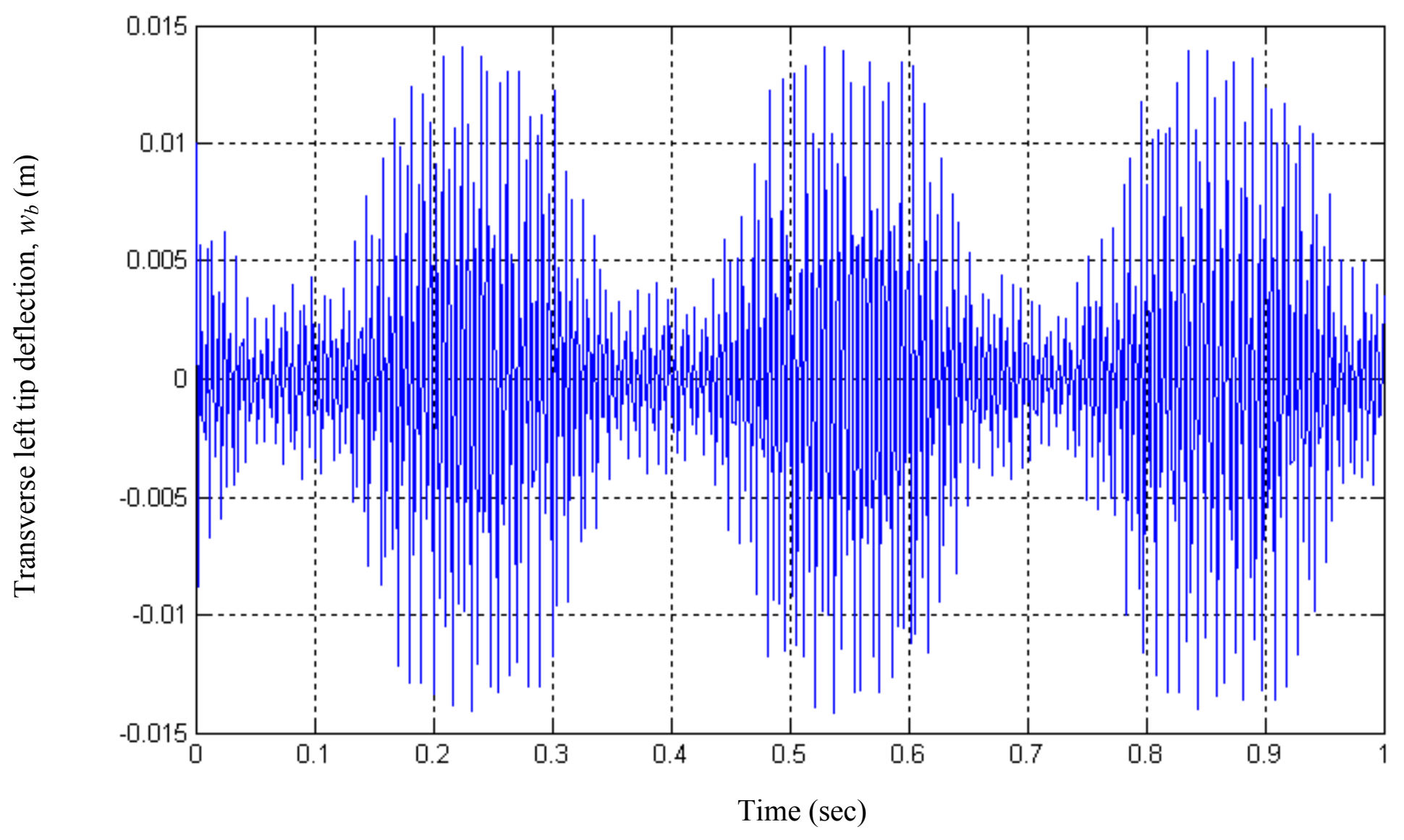

Figure 4.52 Transverse left tip deflection $w_{b}$, HSDT, $\Omega=20 \mathrm{rad} / \mathrm{sec}$ 
Lay-up $=0 / 45 /-45$

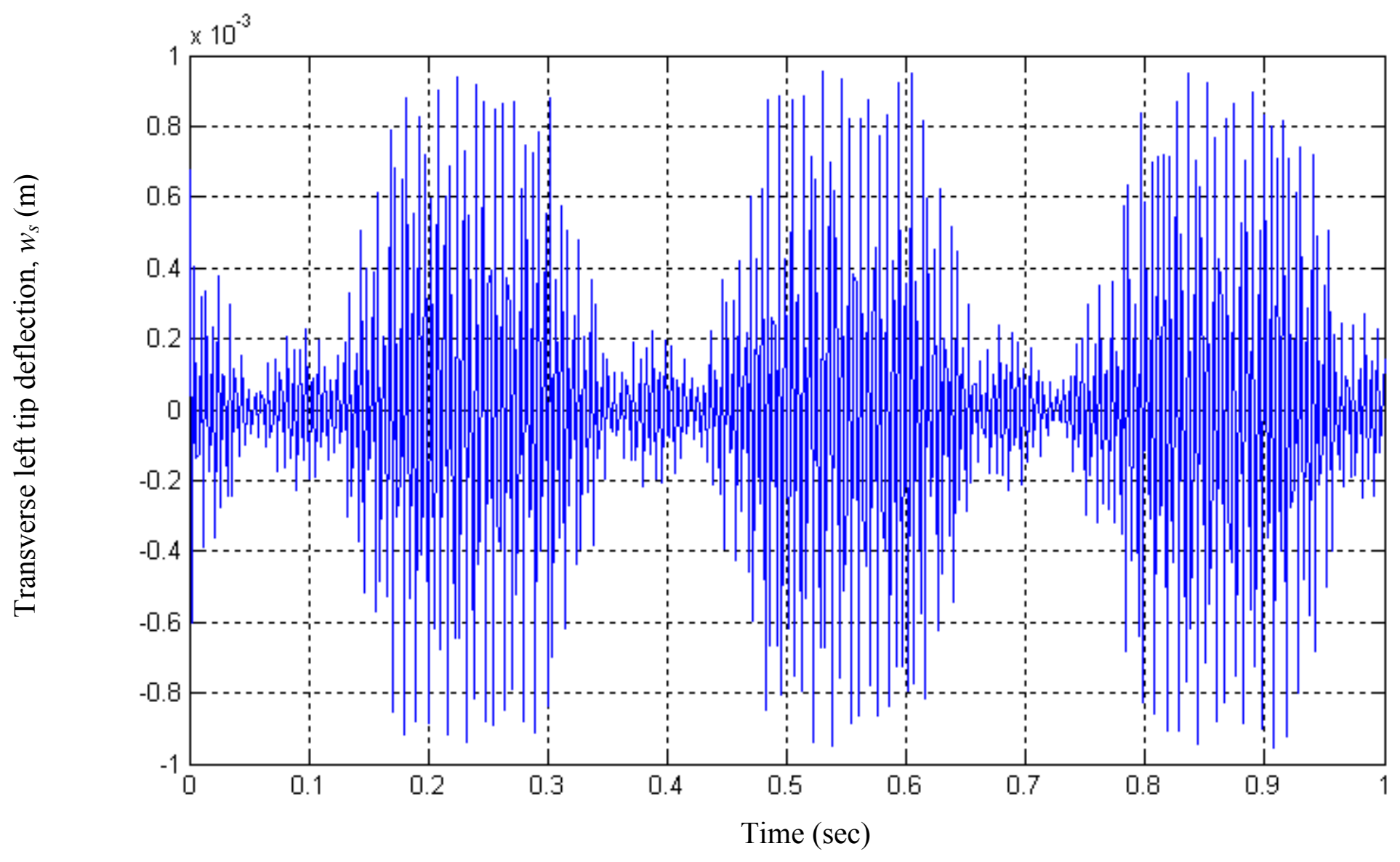

Figure 4.53 Transverse left tip deflection $w_{s}$, HSDT, $\Omega=20 \mathrm{rad} / \mathrm{sec}$ 
Lay-up $=0 / 45 /-45$

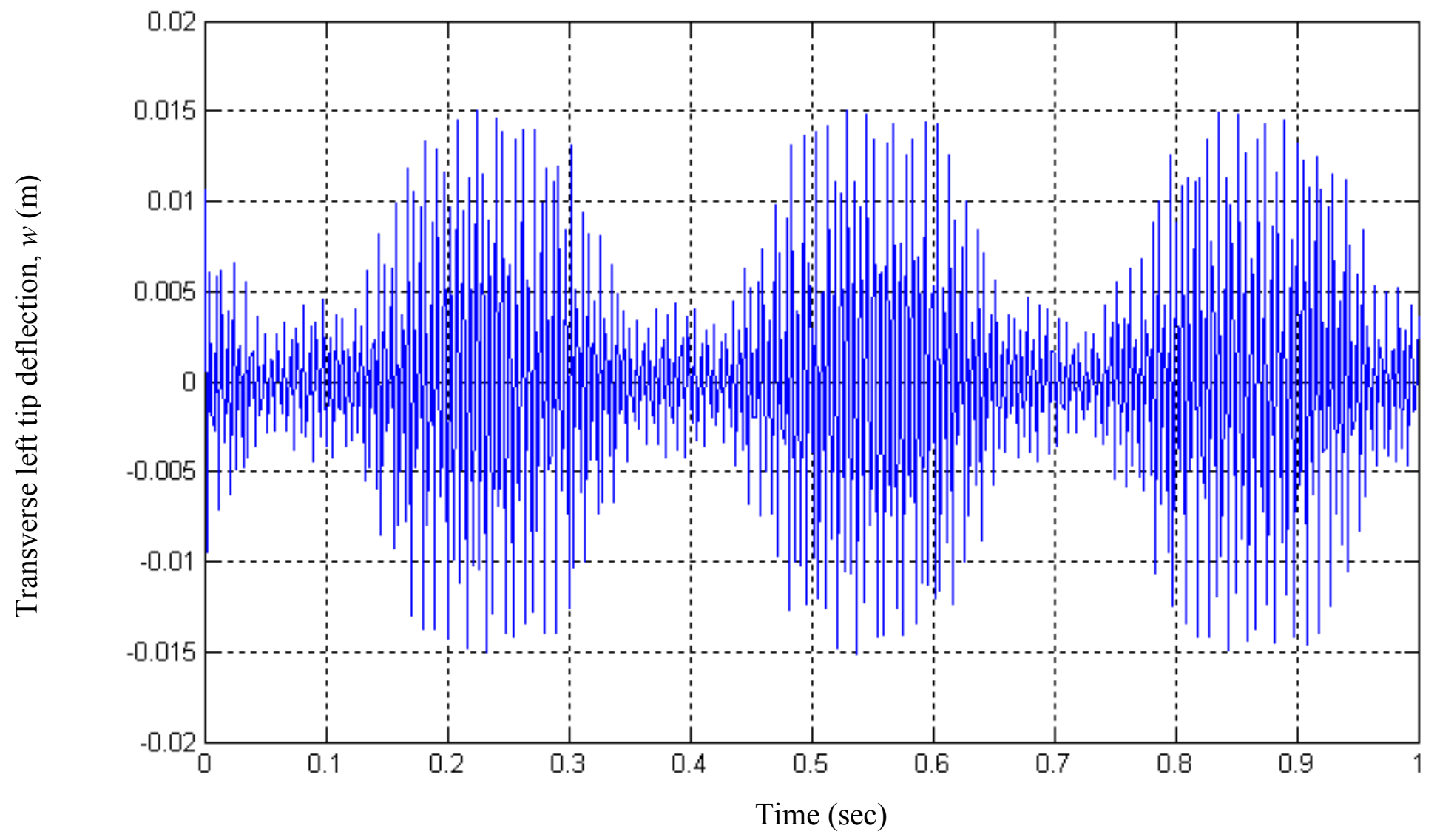

Figure 4.54 Transverse left tip deflection $w$, HSDT, $\Omega=20 \mathrm{rad} / \mathrm{sec}$ 


$$
\text { Lay-up }=0 / 45 /-45
$$

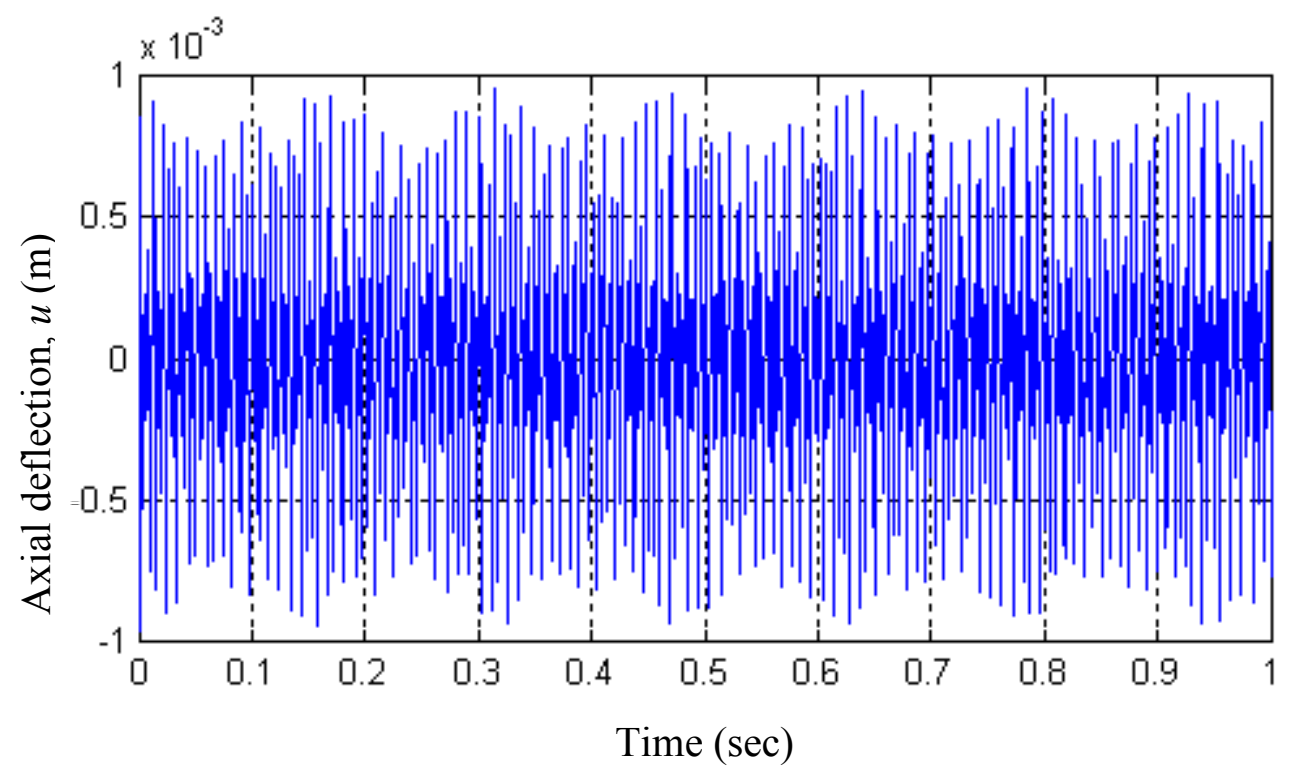

Figure 4.55 Axial right tip deflection $u$, HSDT, $\Omega=20 \mathrm{rad} / \mathrm{sec}$

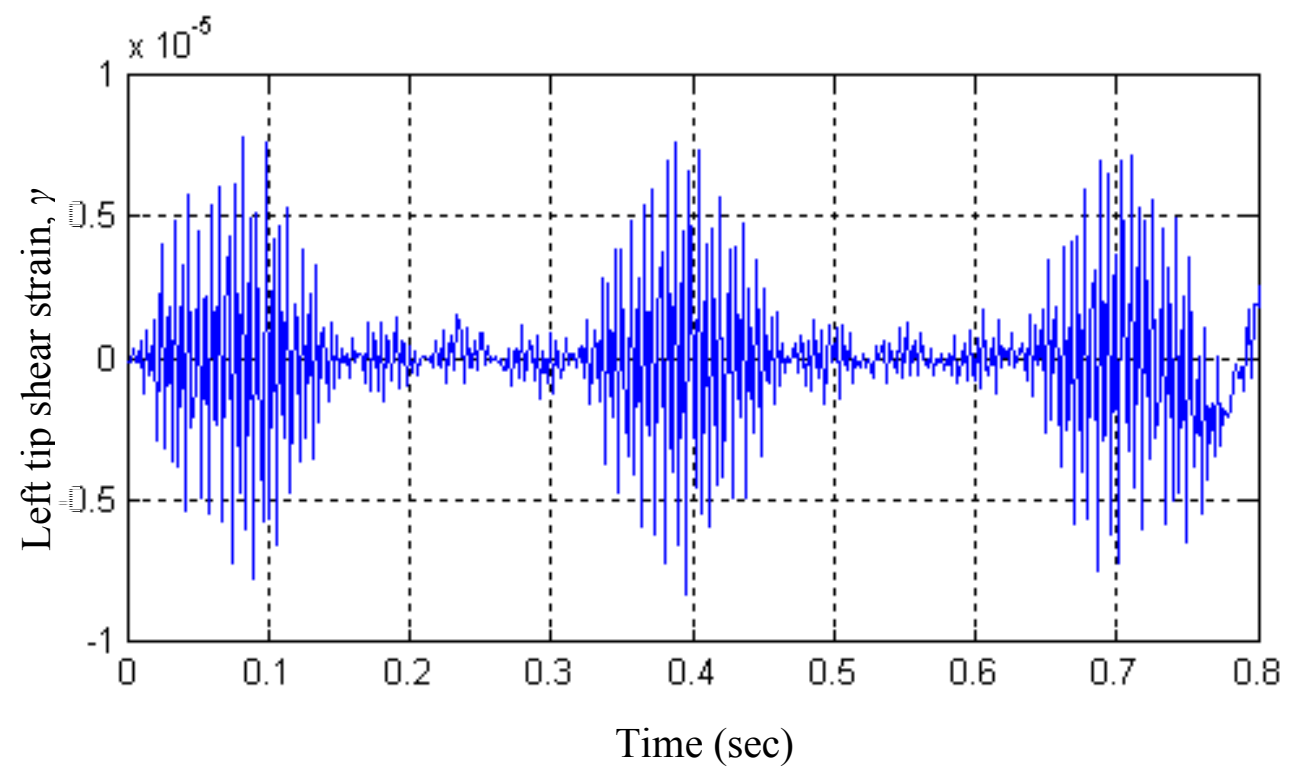

Figure 4.56 Left tip shear strain $\gamma$, HSDT, $\Omega=20 \mathrm{rad} / \mathrm{sec}$ 
Lay-up $=0 / 45 /-45$

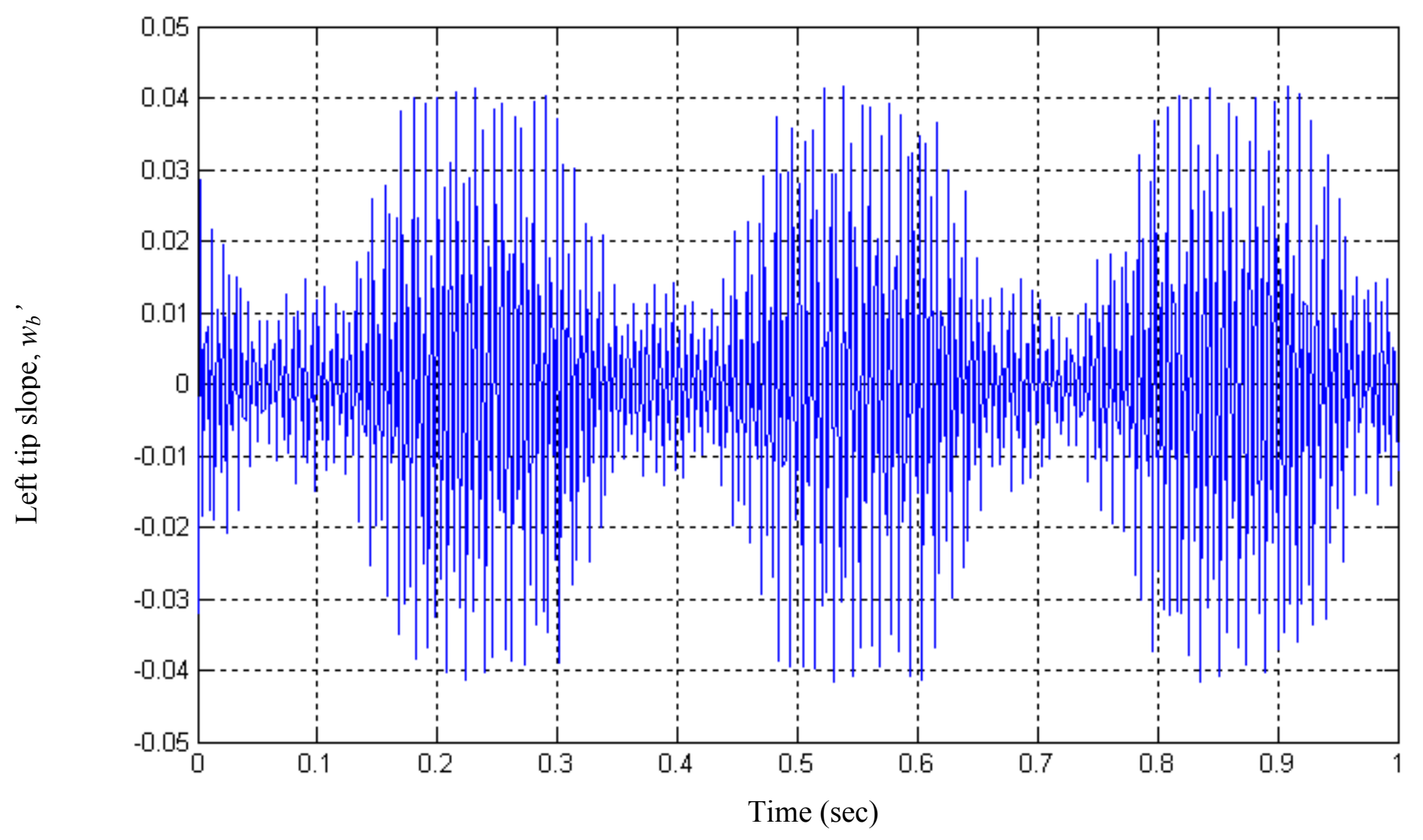

Figure 4.57 Left tip slope $w_{b}$, HSDT, $\Omega=20 \mathrm{rad} / \mathrm{sec}$ 
Lay-up $=0 / 45 /-45$

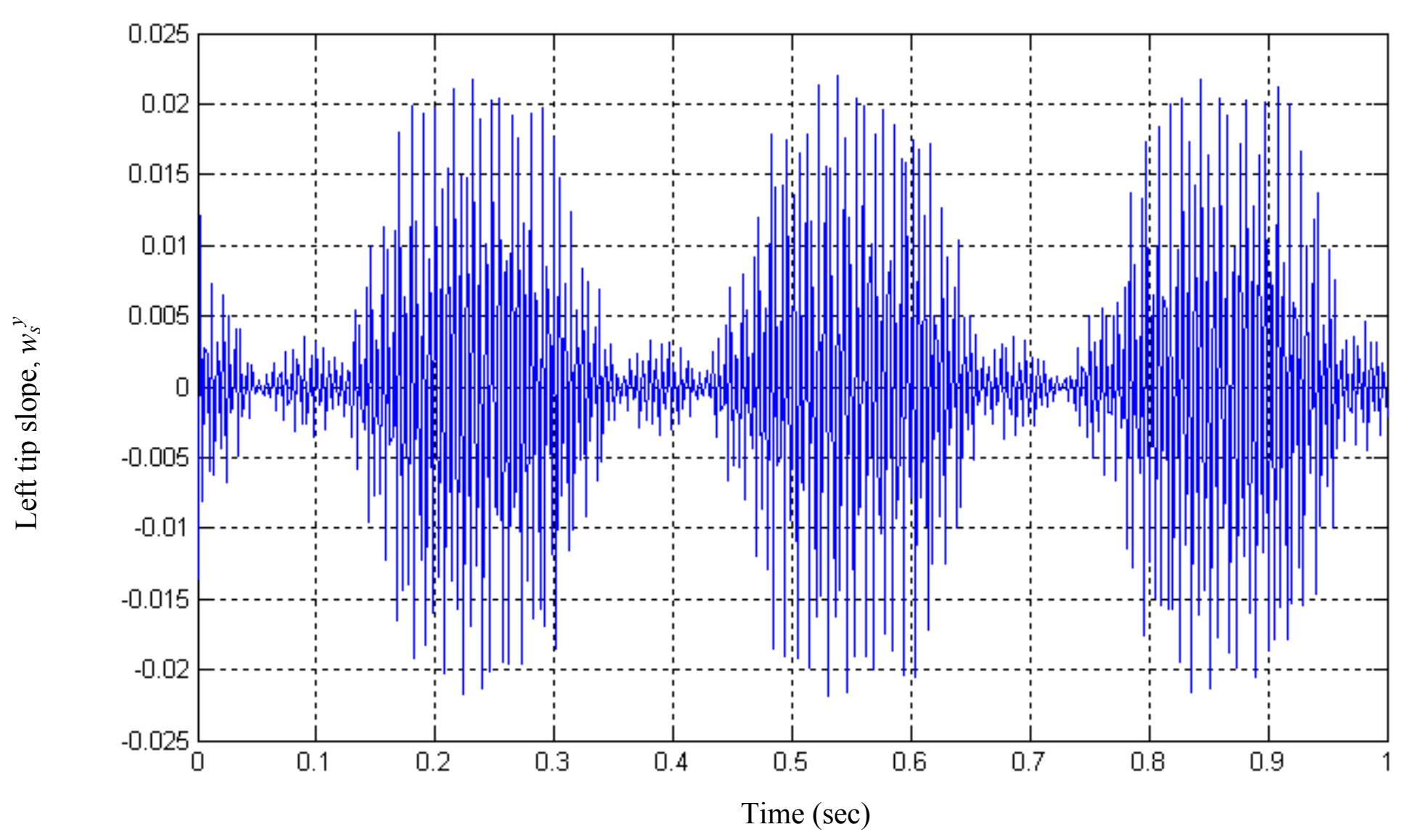

Figure 4.58 Left tip slope $w_{s}^{y}$, HSDT, $\Omega=20 \mathrm{rad} / \mathrm{sec}$ 
Lay-up $=0 / 20 /-20$

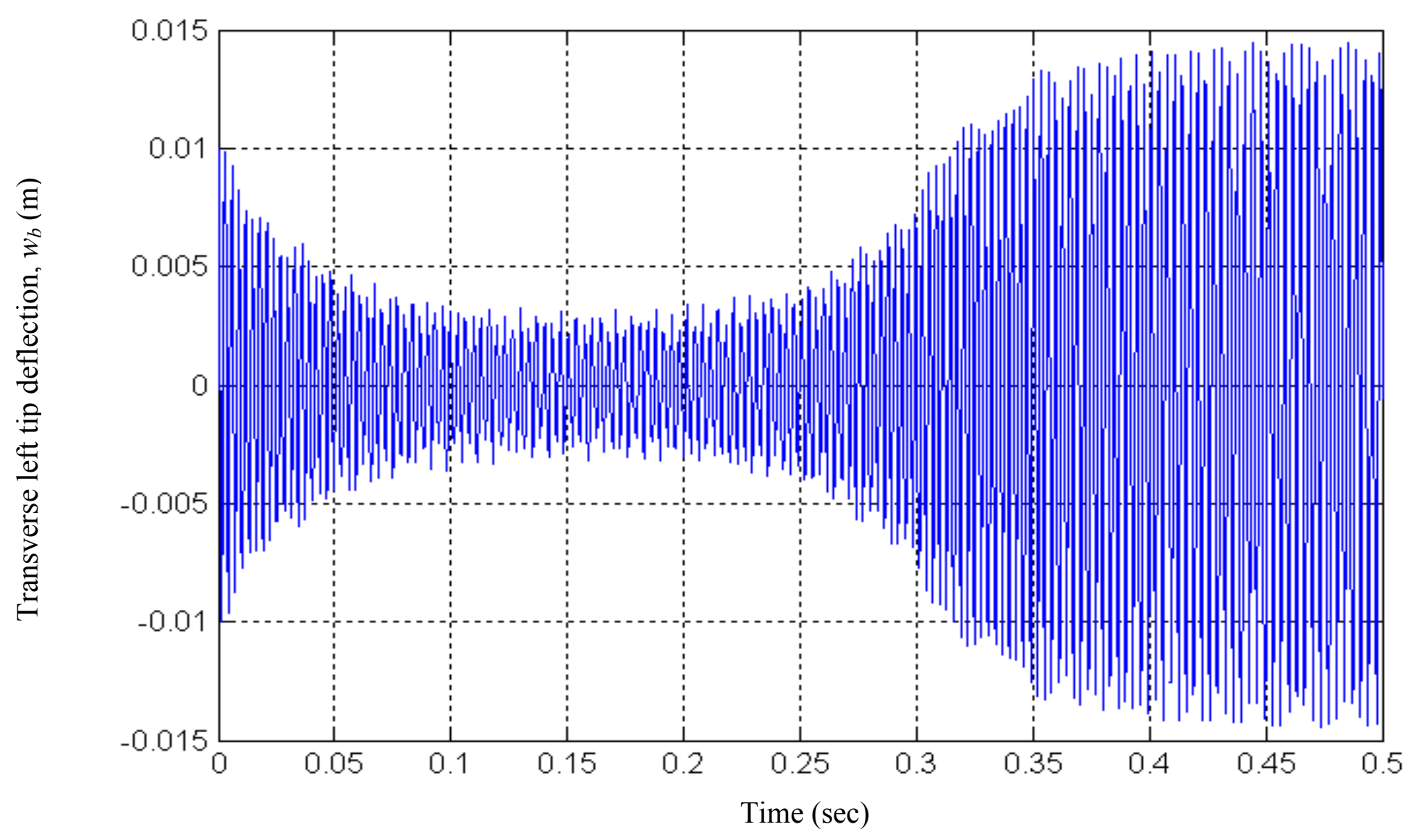

Figure 4.59 Transverse left tip deflection $w_{b}$, HSDT, $\Omega=10 \mathrm{rad} / \mathrm{sec}$ 
Lay-up $=0 / 20 /-20$

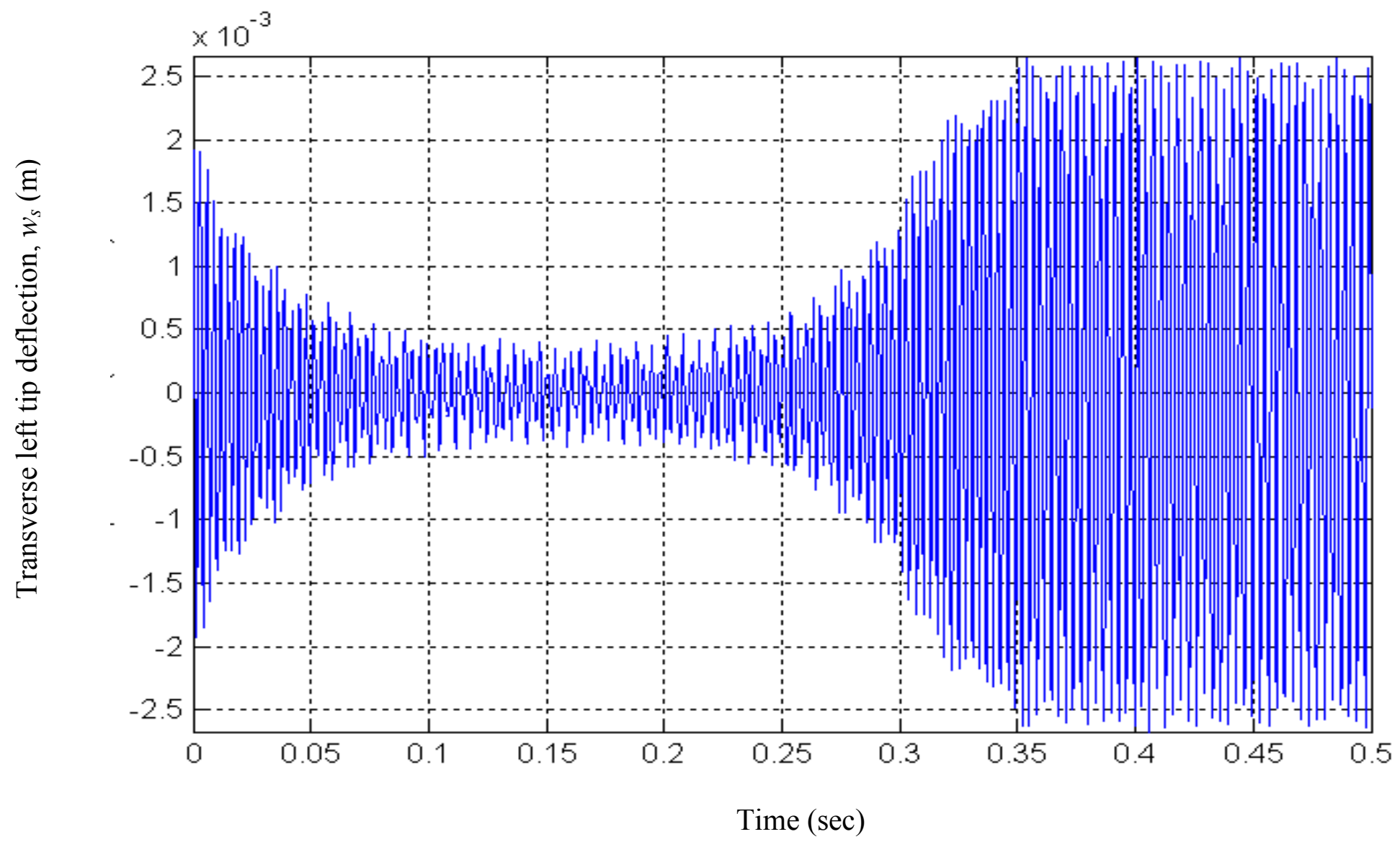

Figure 4.60 Transverse left tip deflection $w_{s}$, HSDT, $\Omega=10 \mathrm{rad} / \mathrm{sec}$ 
Lay-up $=0 / 20 /-20$

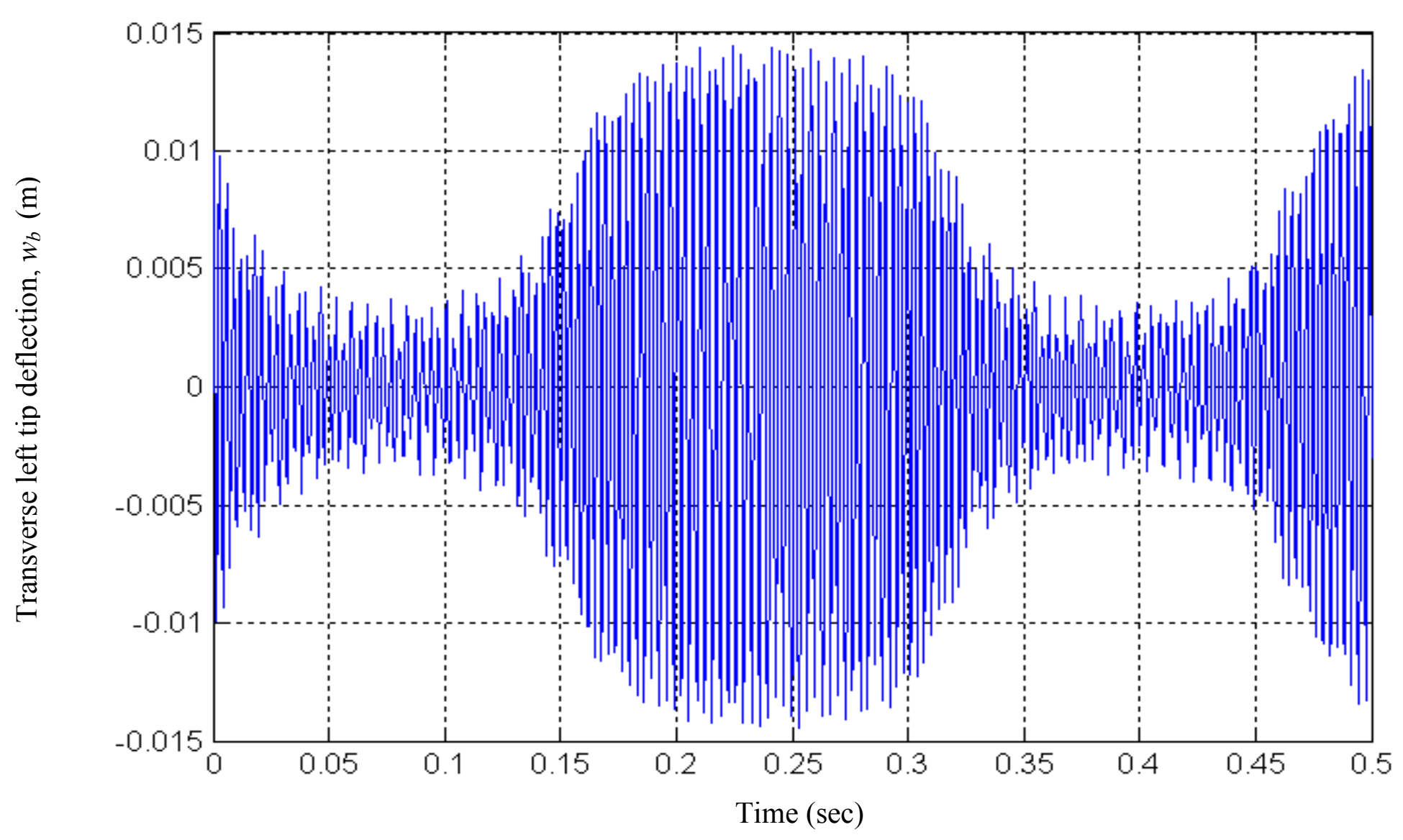

Figure 4.61 Transverse left tip deflection $w_{b}$, HSDT, $\Omega=20 \mathrm{rad} / \mathrm{sec}$ 
Lay-up $=0 / 20 /-20$

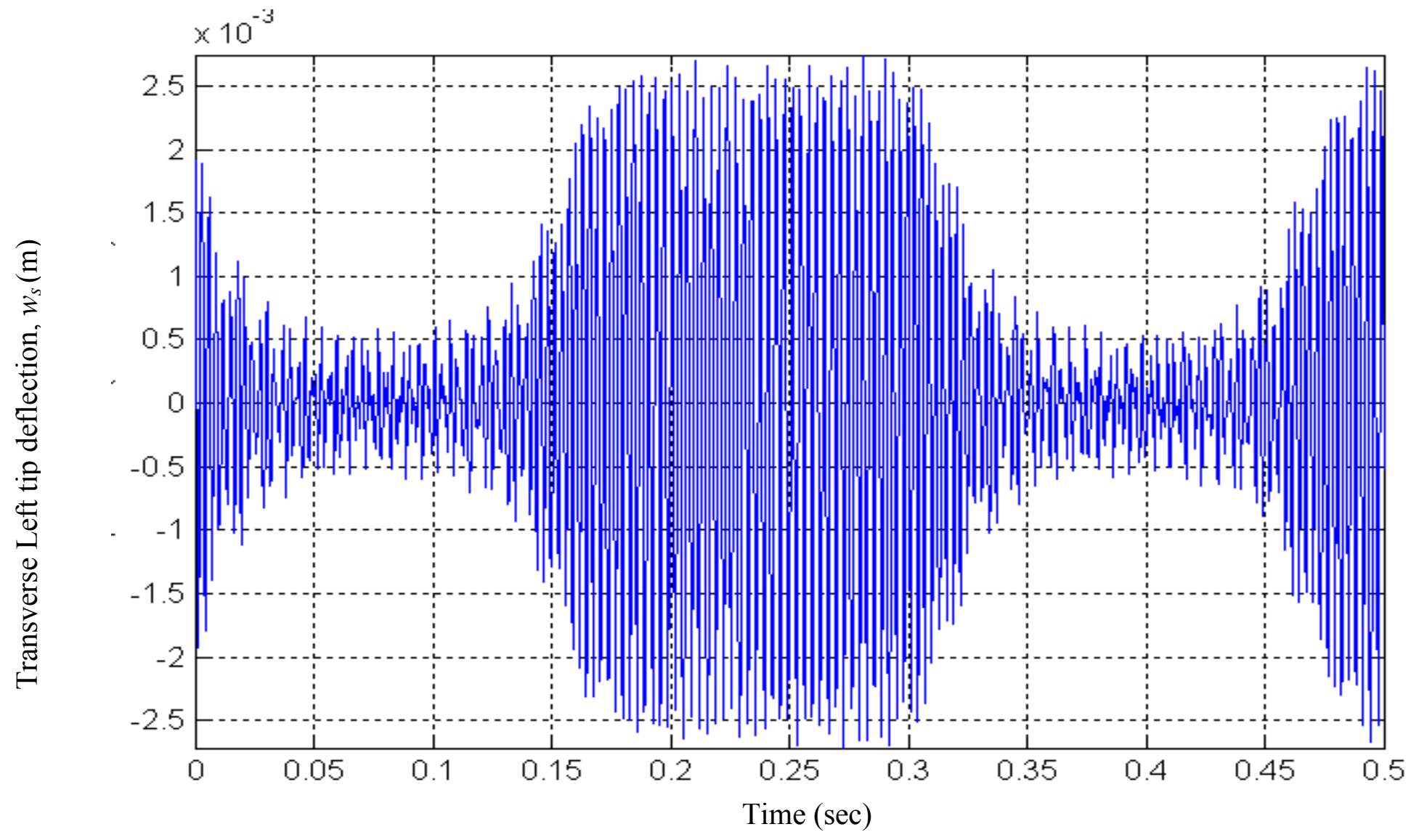

Figure 4.62 Transverse Left tip deflection, $w_{s}$, HSDT, $\Omega=20 \mathrm{rad} / \mathrm{sec}$ 
Lay-up $=0 / 45 /-45 /-45 / 45 / 0$

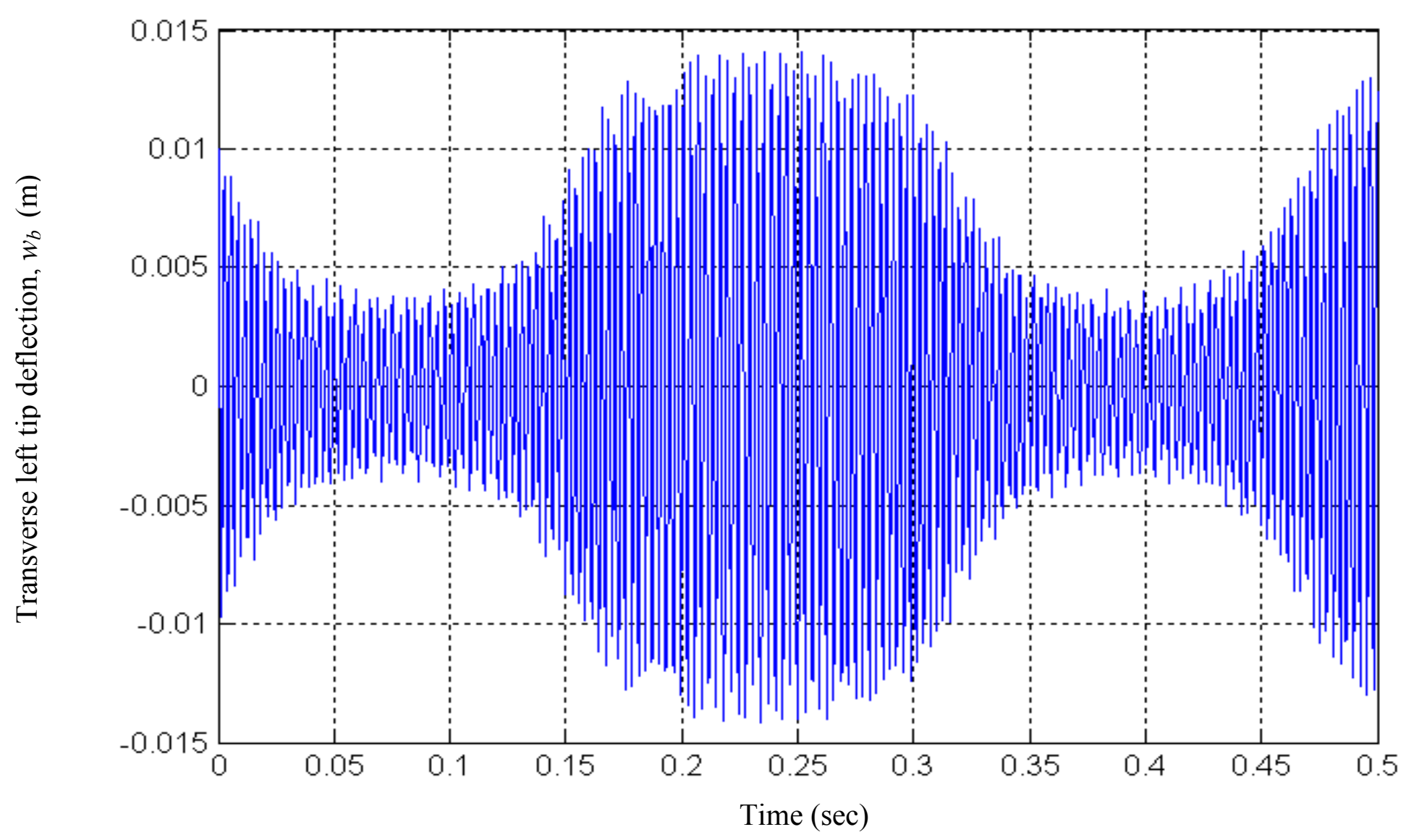

Figure 4.63 Transverse left tip deflection $w_{b}$, HSDT, $\Omega=20 \mathrm{rad} / \mathrm{sec}$ 
Lay-up $=0 / 45 /-45 /-45 / 45 / 0$

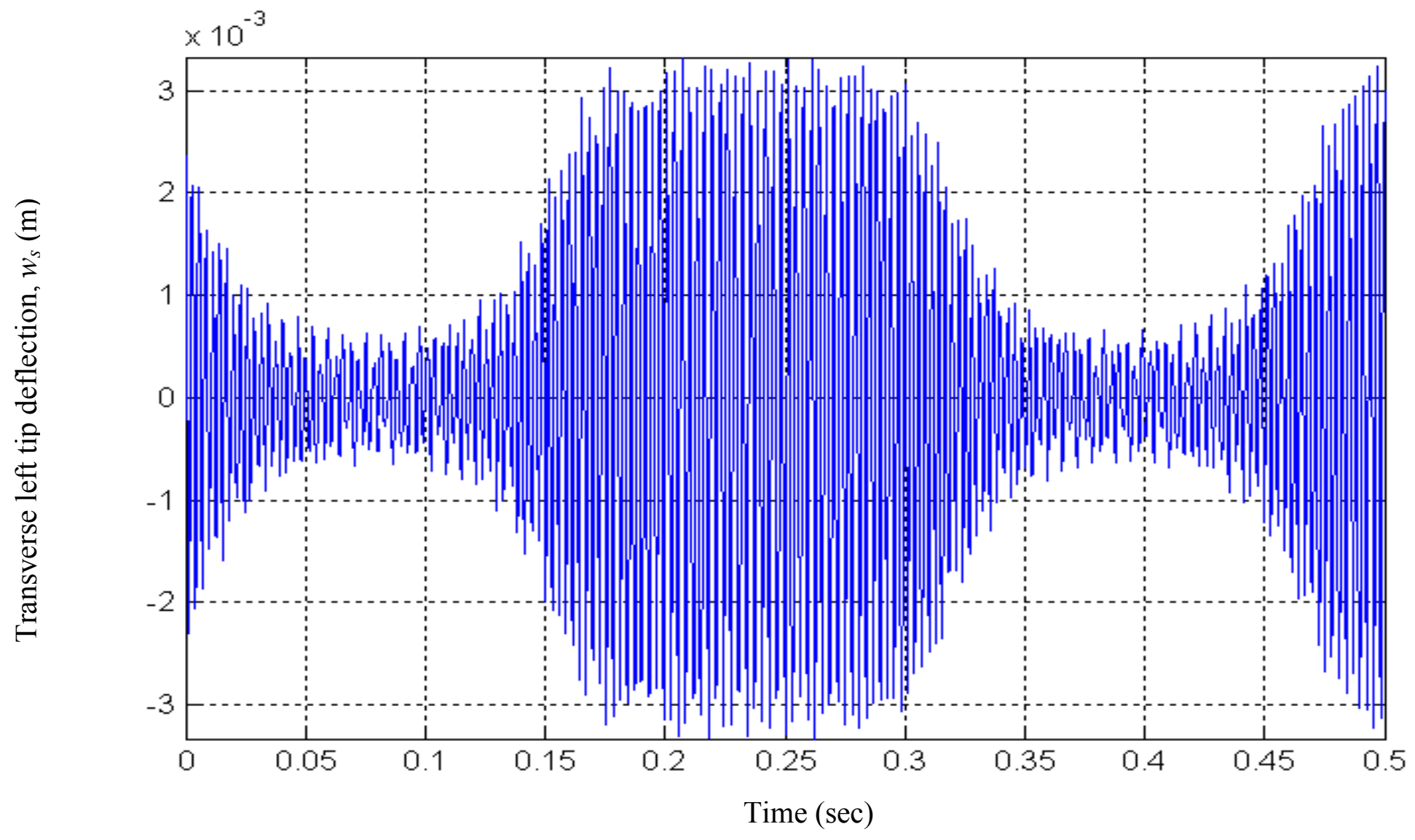

Figure 4.64 Transverse left tip deflection, $w_{s}$, HSDT, $\Omega=20 \mathrm{rad} / \mathrm{sec}$ 


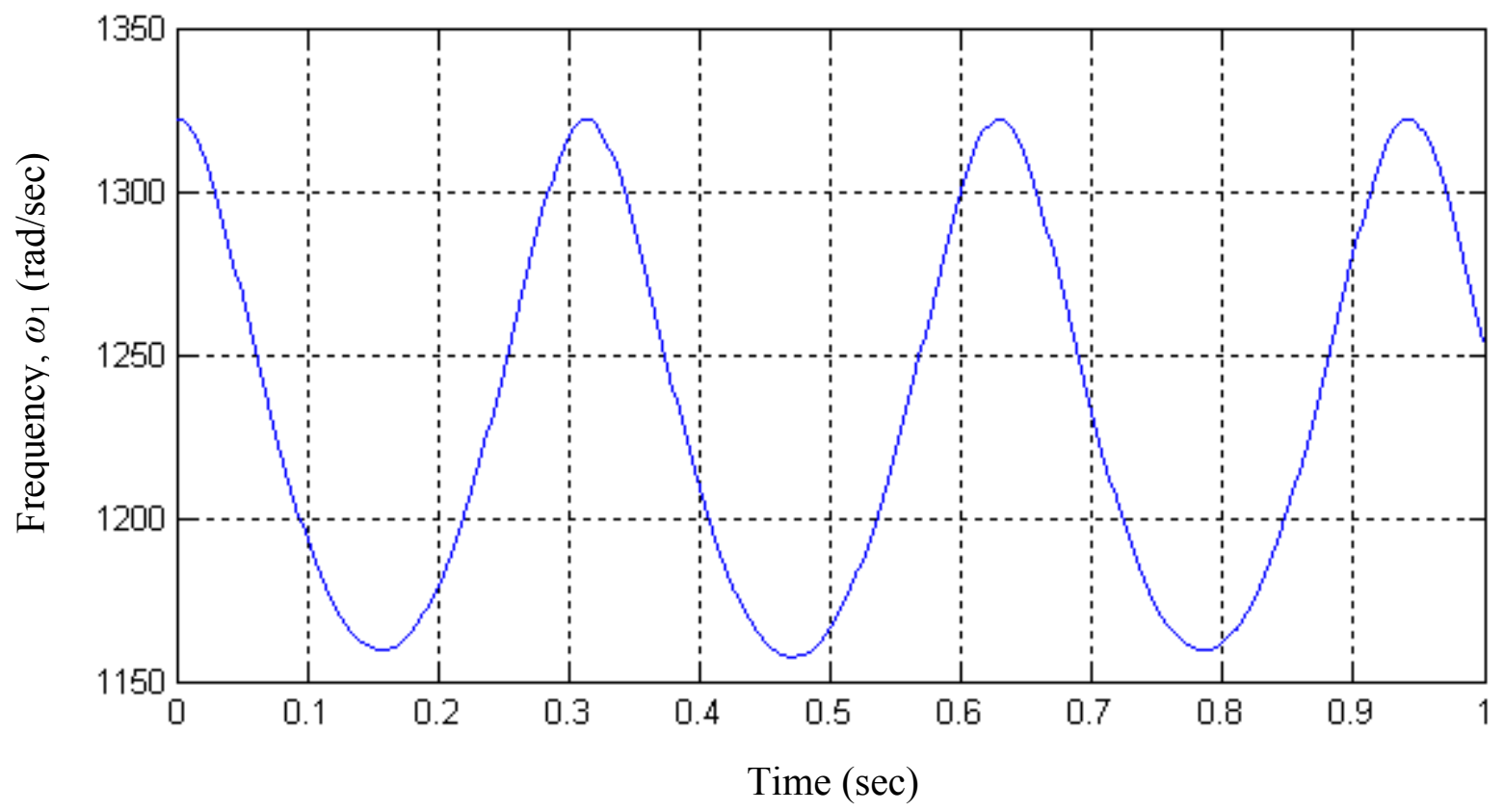

Figure 4.65 First transverse natural frequency $\omega_{1}$, of $[0 / \pm 45]$, HSDT, $\Omega=10 \mathrm{rad} / \mathrm{sec}$

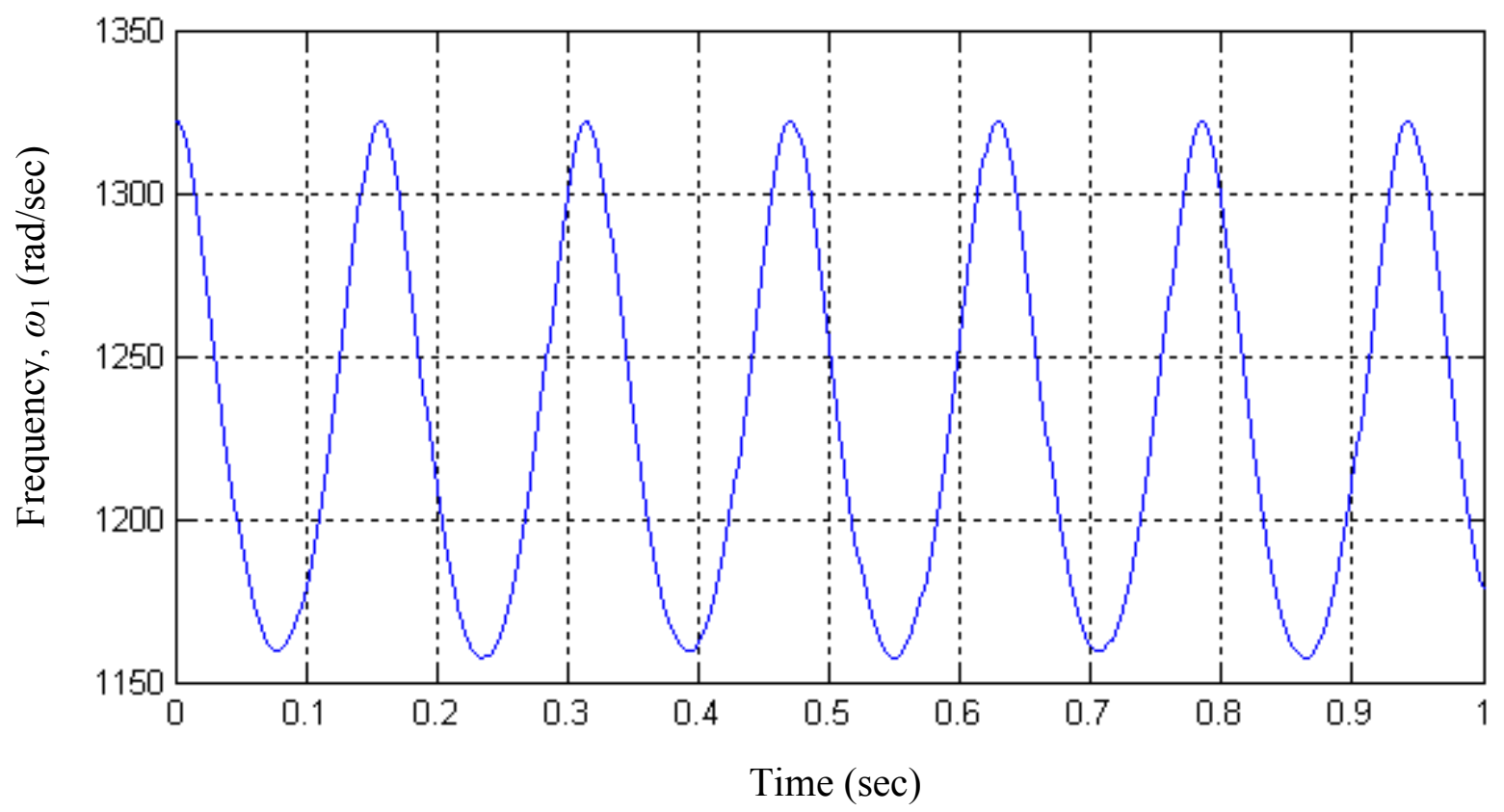

Figure 4.66 First transverse natural frequency $\omega_{1}$, of $[0 / \pm 45]$, HSDT, $\Omega=20 \mathrm{rad} / \mathrm{sec}$ 


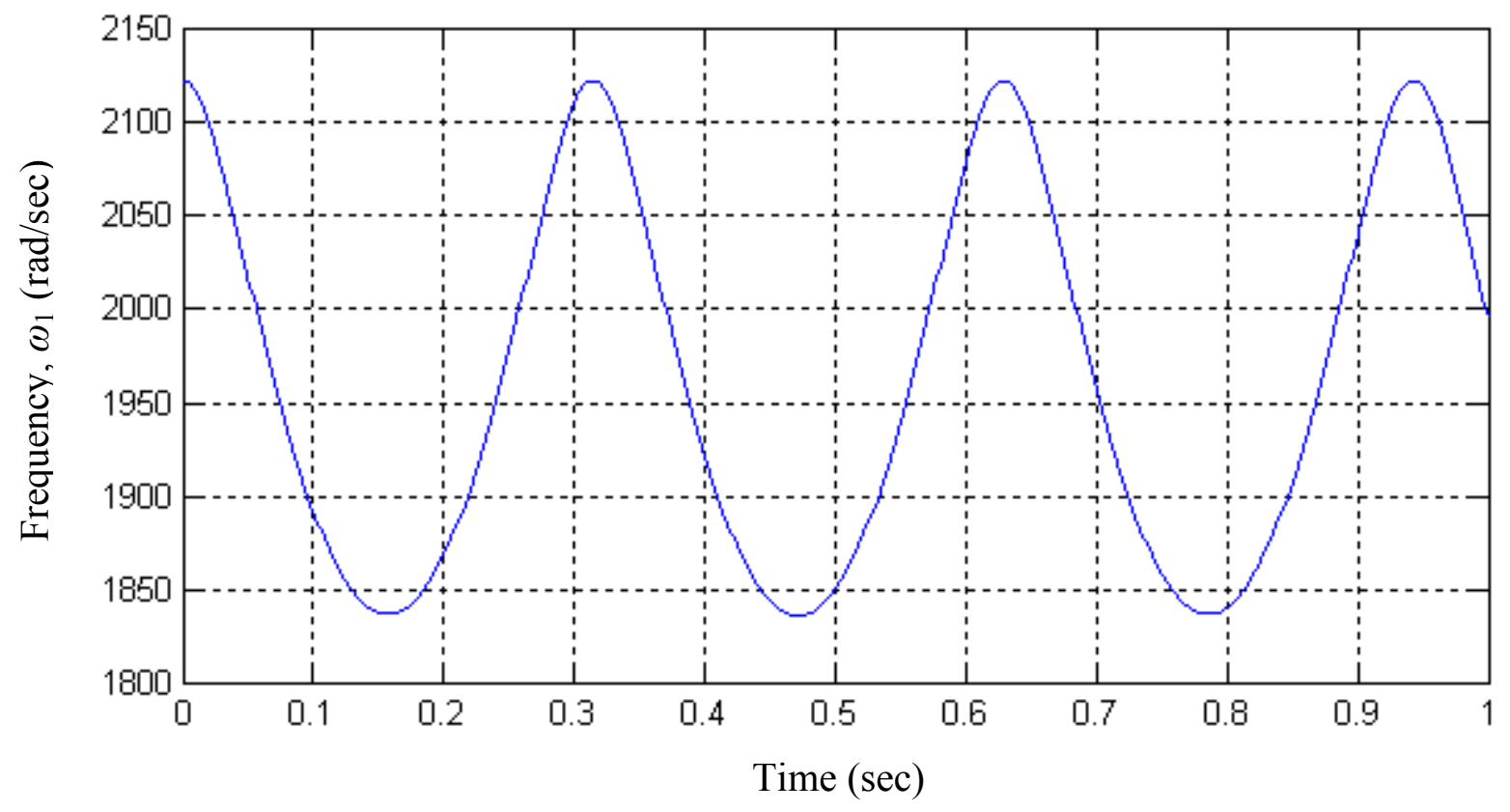

Figure 4.67 First transverse natural frequency $\omega_{1}$, of $[0 / \underline{20}]$, HSDT, $\Omega=10 \mathrm{rad} / \mathrm{sec}$

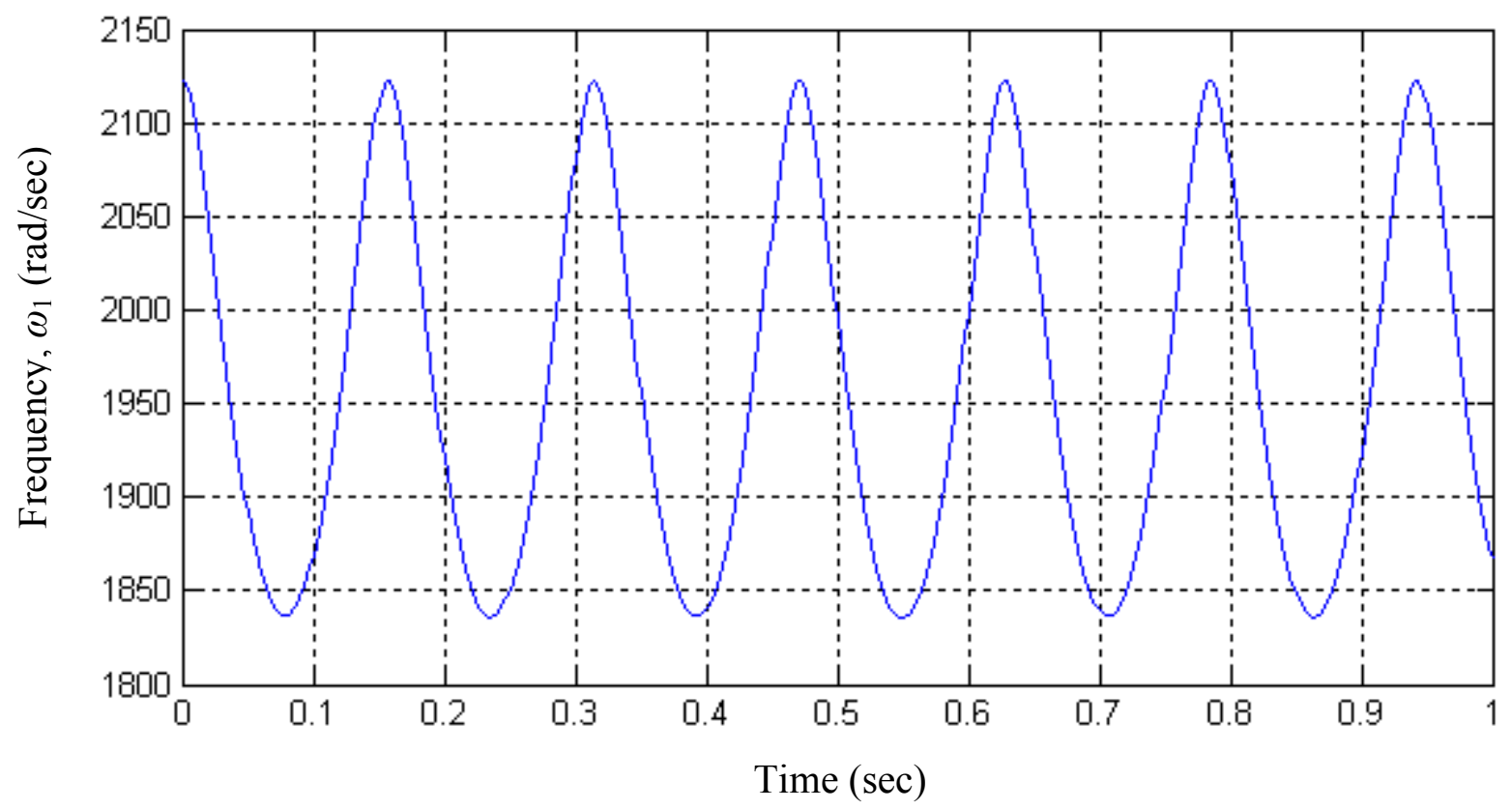

Figure 4.68 First transverse natural frequency $\omega_{1}$, of $[0 / \pm 20]$, HSDT, $\Omega=20 \mathrm{rad} / \mathrm{sec}$ 


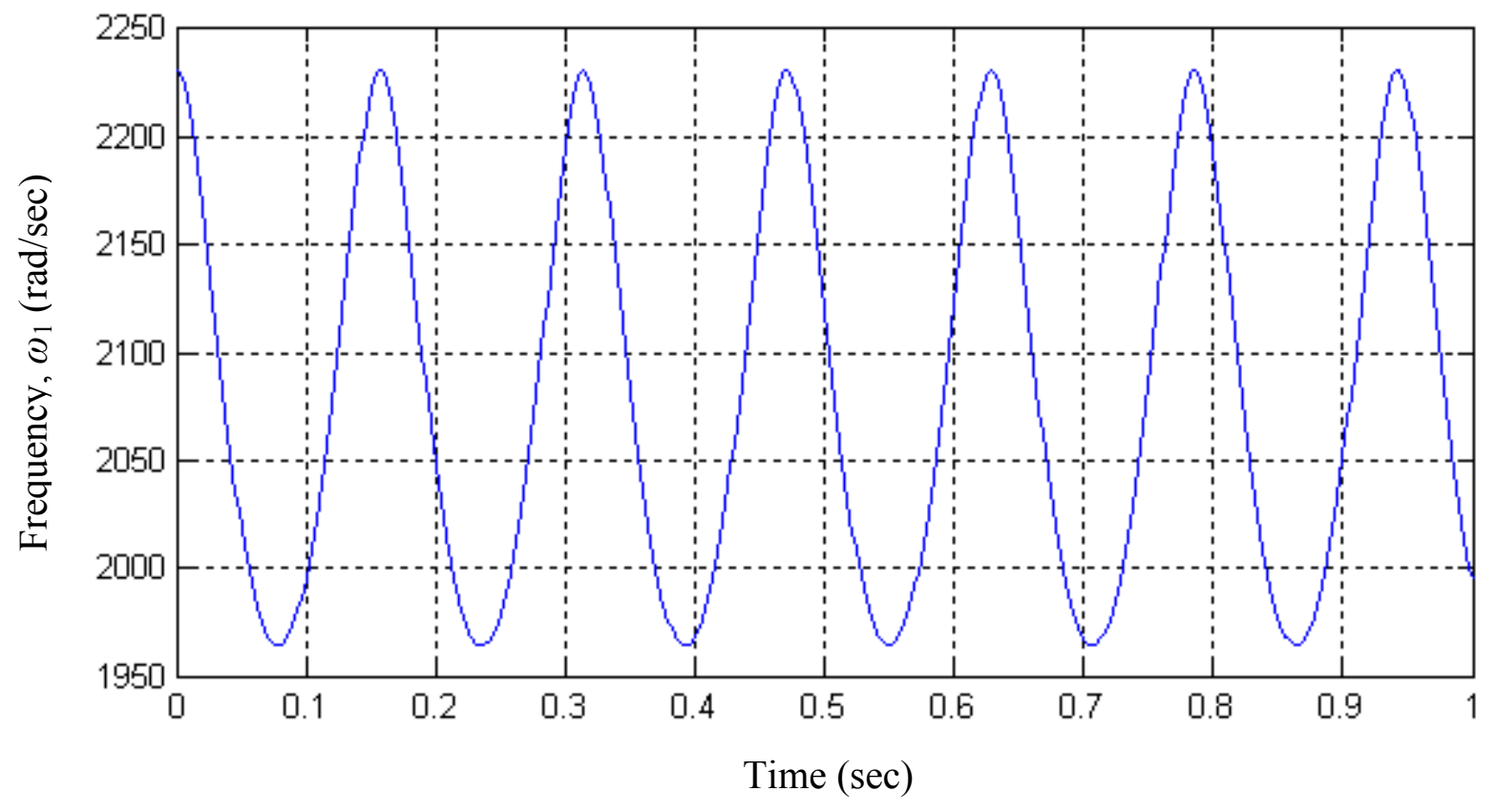

Figure 4.69 First transverse natural frequency $\omega_{1}$, of $[0 / \underline{+45}]_{\mathrm{s}}$ HSDT, $\Omega=10 \mathrm{rad} / \mathrm{sec}$ 


\section{CONCLUSIONS AND RECOMMENDATIONS}

\subsection{Contributions}

1. A higher-order finite element model with five nodes and thirty-four degrees of freedom is presented for the analysis of a composite moving beam based on HSDT.

2. The beam element is developed using Lagrange polynomial functions with $C^{0}$ continuity for some variables and Hermite polynomial functions with $C^{1}$ continuity for the other variables.

3. The essential conditions for the composite moving beam are introduced into the system by Lagrange multipliers.

4. A MATLAB code is written to solve the finite element equations. The time response of the composite moving beam is carried out using Newmark's method and Wilson Theta method.

5. The beam analysis is carried out incorporating the complete composite properties instead of reducing it into an equivalent isotropic beam. 


\subsection{Conclusions}

1. An $h-p$ version, one dimensional finite element is developed and successfully applied for the analysis of symmetrically and unsymmetrically laminated composite moving beams using higher-order shear deformation theory.

2. The essential constraints are successfully applied via Lagrange multipliers. This method is found to be very effective for such problems with time dependent boundary conditions where support locations change with time and do not fall exactly on the nodes. If conventional finite element approach was used, new node locations needed to be created at each time step. Using the Lagrange multiplier method, the additional steps of remeshing at every time step is avoided.

3. A beat like phenomenon is observed in the response of CLPT, FSDT, and HSDT beams. The reason for this is the interplay of the forcing axial frequency (low) with the first transverse natural frequency (high). An increase in the axial frequency results in a corresponding increase in the the number of beats.

4. The fundamental frequencies obtained using HSDT for non-moving beams with small slenderness ratios are higher compared to the corresponding results obtained using FSDT. For beams with large slenderness ratio the fundamental frequencies obtained using FSDT and HSDT are nearly the same.

5. The transverse bending deflections obtained using HSDT is similar to that of the FSDT case, but the transverse shear deflections are different. The amplitude of the transverse shear deflections are larger than that from FSDT.

6. While the magnification factor due to the moving beam effect was about 1.4 for all the three theories of CLPT, FSDT, and HSDT, there were slight variations 
among them. The magnification factors for the FSDT examples are lower than the corresponding CLPT ones. The HSDT examples exhibited slightly lower magnification factors compared to the corresponding FSDT ones.

\subsection{Recommnedations}

1. The response of the moving beam can be studied by introducing damping in the model.

2. The theoretical results can be verified experimentally.

3. Nonlinear analysis based on large defection theory can be performed. 


\section{REFERENCES}

Barbero, E.J., 1998, “Introduction to Composite Materials Design,” Taylor and Francis, Philadelphia.

Bathe, K.J., 2001, “Finite Element Procedures," Fifth Edition, Prentice-Hall of India Private Ltd., New Delhi, India.

Buffington, K.W. and Kane, T.R., 1985, "Dynamics of a Beam Moving Over Supports," International Journal of Solids and Structures, Vol. 21, No. 7, pp. 617-643.

Chandrasekaran, G., 2000, "Dynamic Analysis of a Composite Moving Beam," Master's Thesis, West Virginia University, Morgantown, WV.

Chen, A.T and Yang, T.Y., 1985, "Static and Dynamic Formulation of a Symmetrically Laminated Beam Finite Element for a Microcomputer,” Journal of Composite Materials, Vol. 19, pp. 459-475.

Desai, Y.M. and Ramtekkar, G.S., 2002, "Mixed finite element model for laminated composite beams," Structural Engineering and Mechanics, Vol. 13, No. 3, pp. 261-276.

Kadivar, M.H. and Mophebpour, S.R., 1997, "Forced Vibration of Unsymmetric Laminated Composite Beams under the action of Moving Loads," Journal of composites Science and Technology, Vol. 58, No. 10, pp. 1675-1684.

Kadivar, M.H. and Mohebpour, S.R., 1998, "Finite Element Dynamic Analysis of Unsymmetric Composite Laminated Beams with Shear Effect and Rotary Inertia under the action of Moving Loads," Finite elements in Analysis and Design, Vol. 29, pp. 259273. 
Kapania, R.K. and Raciti, S., 1989a, "Nonlinear Vibrations of Unsymmetrically Laminated Beams," AIAA Journal, Vol. 27, No. 2, pp. 201-210.

Kapania, R.K. and Raciti, S., 1989b, "Recent advances in analysis of laminated beams and plates. Part II. Vibrations and wave propagation," Enhancing Analysis Techniques for Composite Materials, NDE-Vol. 10, pp. 159-168.

Kapania, R.K. and Singhvi, S., 1991, "Free Vibration Analysis of Generally Laminated Tapered Skew Plates," AIAA Journal, Vol. 27, No. 2, pp. 201-210.

Lee H.P., 1992, "Dynamics of a Beam Moving over Multiple Supports," International Journal of Solids and Structures, Vol. 30, No. 2, pp. 199-209.

Lee, I., and Lee, J.J., 1990, "Vibration analysis of a composite plate wing," Journal of Computers and Structures, Vol. 36, No. 6, pp. 1077-1085.

Marur, R.S., and Kant, T., 1998, “Transient Dynamics of Laminated Beams: an Evaluation with Higher-Order Refined Theories," Journal of Composite Structures, Vol. 41, pp. 1-7.

Reddy, J.N., 1985, “ Review of the Literature on Finite-Element Modeling of Laminated Composite Plates," Journal of Shock and Vibration Digest, Vol. 17, No. 4, pp. $3-8$.

Reddy, J.N., 1997, "Mechanics of Laminated Composite Plates: Theory and Analysis," CRC Press, Boca Raton, Fla.

Shi, G., Lam, K.Y., and Tay, T.E., 1998, “On efficient finite element modeling of composite beams and plates using higher-order theories and accurate composite beam element," Composite Structures, Vol. 41, No. 2, pp. 159-165. 
Sheng, H.Y. and Ye, J.Q., 2002, “ A state space finite element for laminated composite plates," Computer Methods in Applied Mechanics and Engineering, Vol. 191, No. 37-38, pp.4259-4276.

Singh, G., Rao, G.V., and Iyengar N.G.R., 1991, “Analysis of Nonlinear Vibrations of Unsymmetrically Laminated Composite Beams," AIAA Journal, Vol. 29, No. 10, pp. 1727-1735.

Sreeram, T.R. and Sivaneri, N.T., 1997, "FE-Analysis of a Moving Beam using Lagrangian Multiplier Method," International Journal of Solids and Structures, Vol. 35, No. 28-29, pp. 3675-3694.

Sreeram, T.R., 1995, "Dynamic Analysis of a Moving Beam using h-p version Finite Element Method with Essential Conditions Applied Via Lagrange Multipliers," Master's Thesis, West Virginia University, Morgantown, WV. 


\section{BIBLIOGRAPHY}

Chandrupatla, T.R. and Belegundu D.A., 1997, “Introduction to Finite Elements

in Engineering," Second Edition, Prentice-Hall of India Private Ltd., New Delhi, India.

Ferziger, J.H., 1981, “Numerical methods for engineering application,” Wiley, New York.

Palm, W.J. , 2001, “Introduction To Matlab 6 For Engineers,” First Edition, McGraw-Hill companies, Inc., New York.

Reddy, J.N., 1984, "Energy and variational methods in applied mechanics: with an introduction to the finite element method," Wiley, New York. 